\title{
Parametric Optimization of Visible Wavelength Gold Lattice Geometries for Improved Plasmon-Enhanced Fluorescence Spectroscopy
}

\author{
Casey A. Norville \\ West Virginia University, cnorvill@mix.wvu.edu
}

Follow this and additional works at: https://researchrepository.wvu.edu/etd

Part of the Biomedical Commons, Electromagnetics and Photonics Commons, and the Nanotechnology Fabrication Commons

\section{Recommended Citation}

Norville, Casey A., "Parametric Optimization of Visible Wavelength Gold Lattice Geometries for Improved Plasmon-Enhanced Fluorescence Spectroscopy" (2020). Graduate Theses, Dissertations, and Problem Reports. 7794.

https://researchrepository.wvu.edu/etd/7794

This Thesis is protected by copyright and/or related rights. It has been brought to you by the The Research Repository @ WVU with permission from the rights-holder(s). You are free to use this Thesis in any way that is permitted by the copyright and related rights legislation that applies to your use. For other uses you must obtain permission from the rights-holder(s) directly, unless additional rights are indicated by a Creative Commons license in the record and/ or on the work itself. This Thesis has been accepted for inclusion in WVU Graduate Theses, Dissertations, and Problem Reports collection by an authorized administrator of The Research Repository @ WVU. For more information, please contact researchrepository@mail.wvu.edu. 


\title{
Parametric Optimization of Visible Wavelength Gold Lattice Geometries for Improved Plasmon-Enhanced Fluorescence Spectroscopy
}

\author{
Casey A. Norville, B.S. \\ Thesis Submitted to the \\ Benjamin M. Statler College of Engineering and Mineral Resources \\ at West Virginia University \\ in partial fulfillment of the requirements for the degree of \\ Master of Science \\ in \\ Electrical Engineering \\ Jeremy M. Dawson, Ph.D., Chair \\ David Graham, Ph.D. \\ Dimitris Korakakis, Ph.D. \\ Lane Department of Computer Science and Electrical Engineering

$$
\begin{gathered}
\text { Morgantown, WV } \\
2020
\end{gathered}
$$

Keywords: Biosensors, Enhancement, FDTD, Fluorescence Spectroscopy, Intensity, Optimization, Plasmonics

Copyright @ 2020 Casey A. Norville 


\title{
Abstract \\ Parametric Optimization of Visible Wavelength Gold Lattice Geometries for Improved Plasmon-Enhanced Fluorescence Spectroscopy
}

\author{
Casey A. Norville
}

The exploitation of spectro-plasmonics will allow for innovations in optical instrumentation development and the realization of more efficient optical biodetection components. Biosensors have been shown to improve the overall quality of life through real-time detection of various antibody-antigen reactions, biomarkers, infectious diseases, pathogens, toxins, viruses, etc. has led to increased interest in the research and development of these devices. Further advancements in modern biosensor development will be realized through novel electrochemical, electromechanical, bioelectrical, and/or optical transduction methods aimed at reducing the size, cost, and limit of detection (LOD) of these sensor systems. One such method of optical transduction involves the exploitation of the plasmonic resonance of noble metal nanostructures. This thesis presents the optimization of the electric $(E)$ field enhancement granted from localized surface plasmon resonance (LSPR) via parametric variation of periodic gold lattice geometries using finite difference time domain (FDTD) software. Comprehensive analyses of cylindrical, square, star, and triangular lattice feature geometries were performed to determine the largest surface $E$-field enhancement resulting from LSPR for reducing the LOD of plasmon-enhanced fluorescence (PEF). The design of an optical transducer engineered to yield peak $E$-field enhancement and, therefore, peak excitation enhancement of fluorescent labels would enable for improved emission enhancement of these labels. The methodology presented in this thesis details the optimization of plasmonic lattice geometries for improving current visible wavelength fluorescence spectroscopy. 


\section{Acknowledgements}

I would like to personally thank my academic and research advisor, Dr. Jeremy Dawson. Dr. Dawson has provided me with a plethora of research and social opportunities ranging from employment in his biometric systems laboratory, a graduate research assistantship (GRA) position, multiple conference visitations across the country, and a variety of professional networking opportunities. Dr. Dawson has also served as a mentor to me throughout the entirety of my graduate career. I would like to take the time to preface this section by dedicating this thesis to one of my best friends, Mansford Masters, who was diagnosed with leukemia shortly after the beginning of my graduate program here at West Virginia University. His struggle with, and triumph over, this disease helped illuminate the true potential of my research and the utmost importance of early detection. I also would like to dedicate this thesis to my family beginning with my father, Patrick Norville, my mother, Tami Norville, my sister, Meaghan Norville, and my niece, Harper Willis, for their constant love and support during my time here at West Virginia University. Additionally, I would like to dedicate this thesis to my girlfriend, Nicole Richetelli, for her continual encouragement and motivation through the emotional ups and downs of my graduate experience. I would like to extend special thanks to Kyle Smith for his help as a colleague, friend, and mentor during my journey as a graduate student. Kyle Smith has been pivotal in both my academic and personal development during the entirety of my graduate studies. Also, I would like to extend my thanks to Alex Dillelo, Adam Duncan, Akshitha Gadde, Harley Hart, Kenney Hite, Anand Kadiyala, Douglas Kerr, Yasser Khouj, Seth Leffel, Catherine O'Hearn, Christa Robison, Jake Rose, Stallone Sabatier, Jianbo Sun, Morgan Trester, and Bert Wieliczko. I would also like to thank David Galvan from the University of Washington for his help at the beginning of my graduate career in learning the basics of Lumerical's FDTD Solutions. Finally, I would also like

to express my sincerest gratitude to Chris Randall for his advisory expertise over the past eight years. 


\section{Table of Contents}

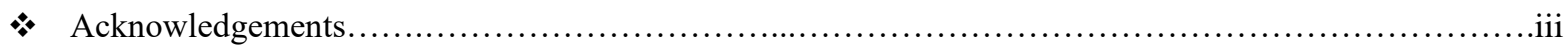

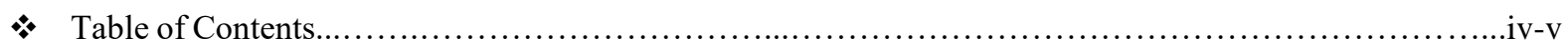

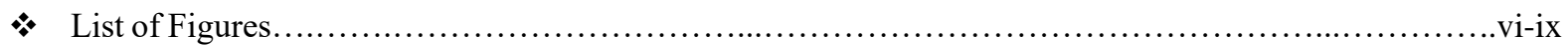

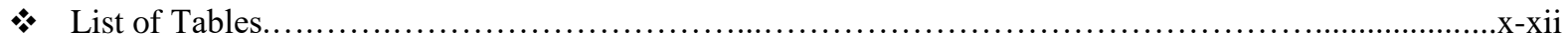

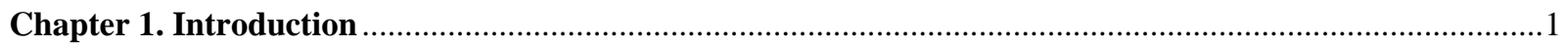

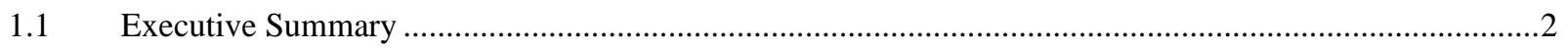

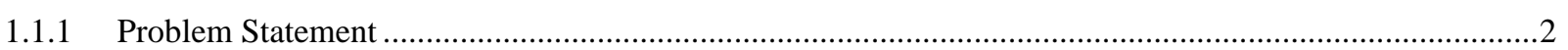

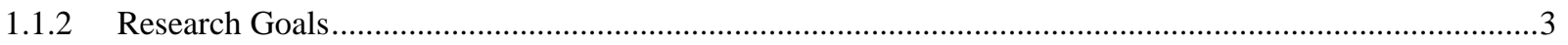

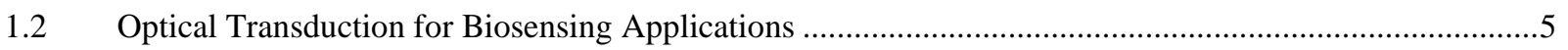

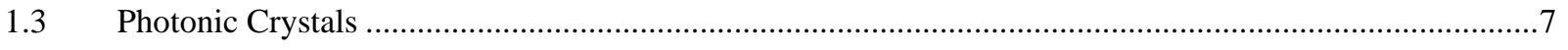

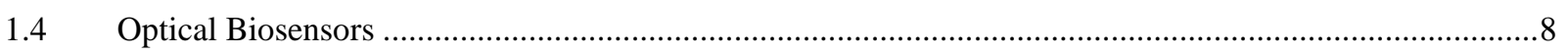

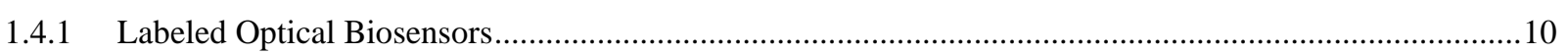

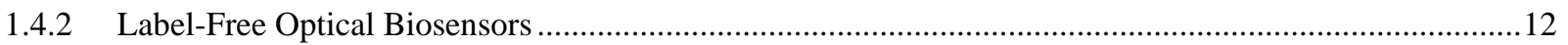

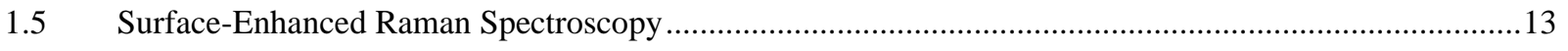

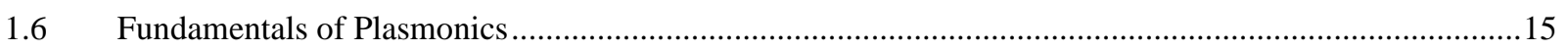

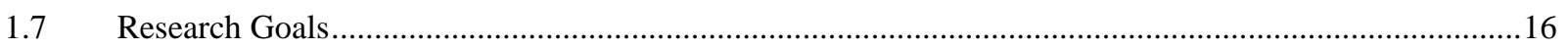

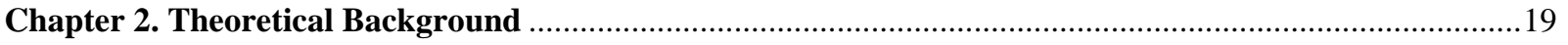

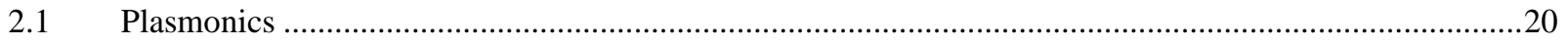

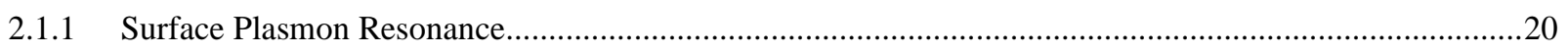

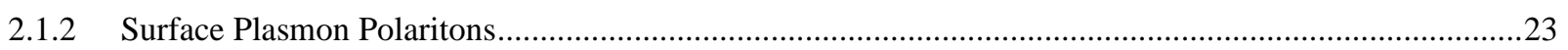

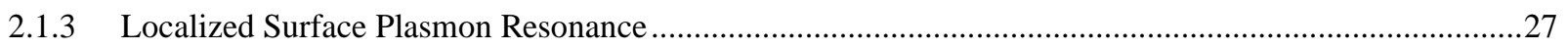

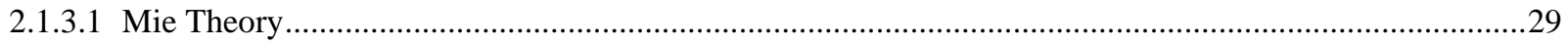

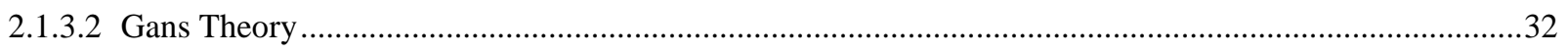

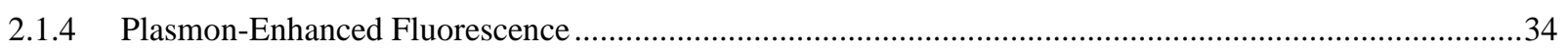

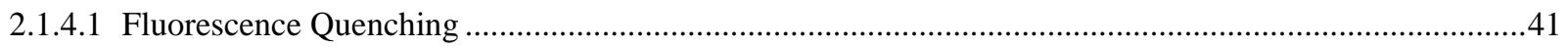

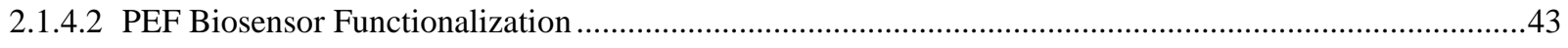

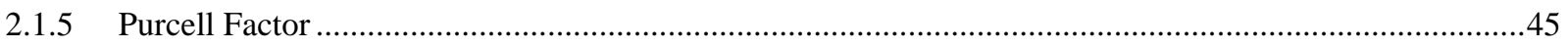

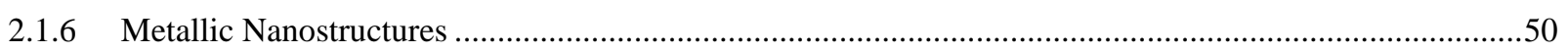

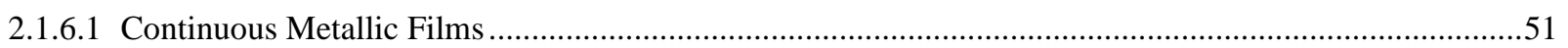

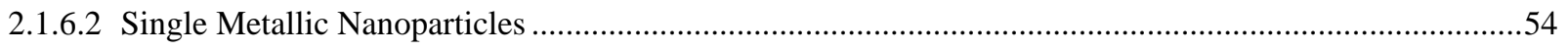

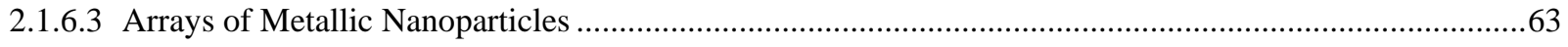

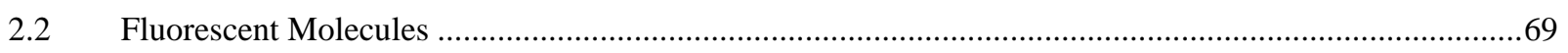

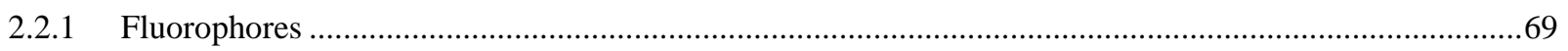

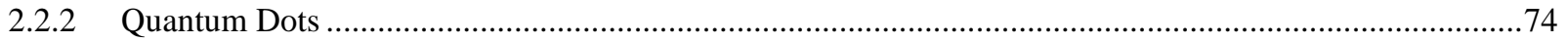




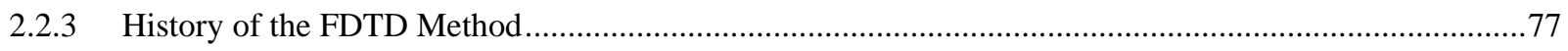

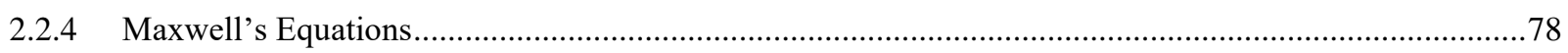

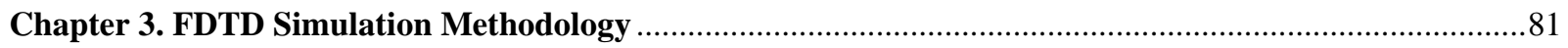

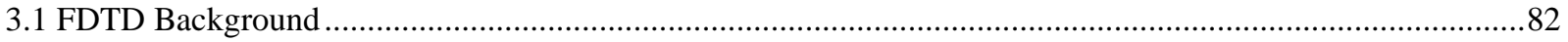

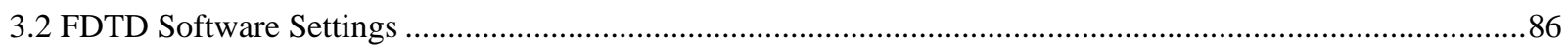

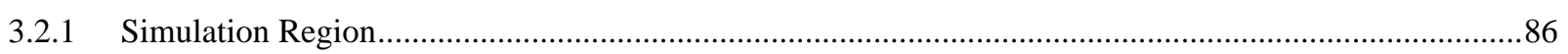

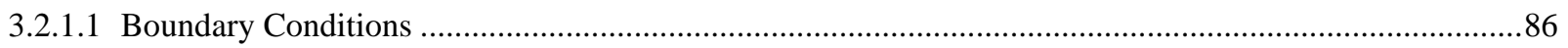

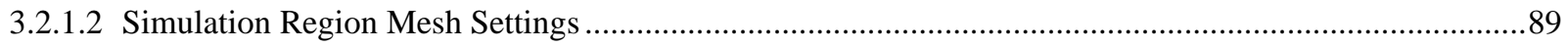

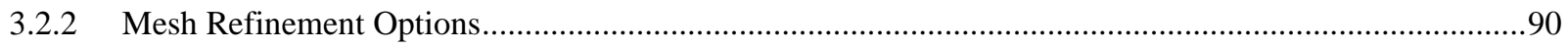

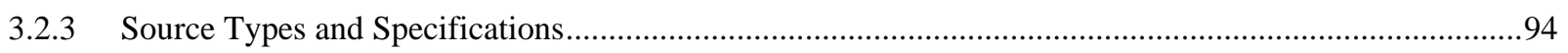

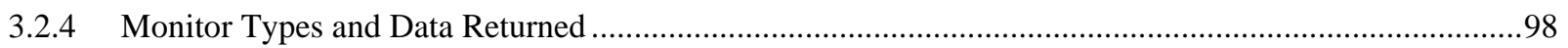

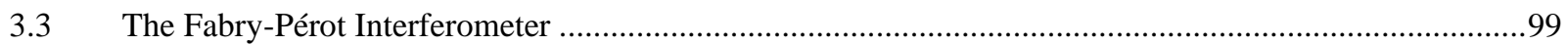

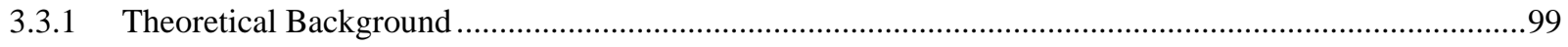

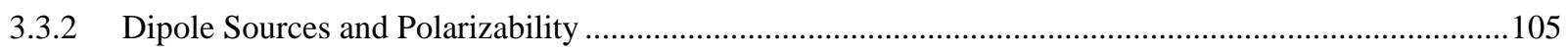

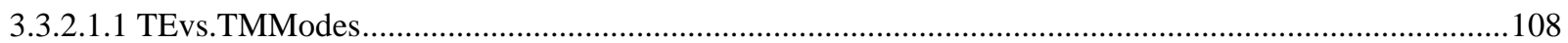

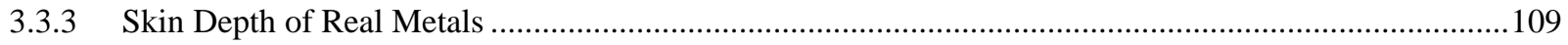

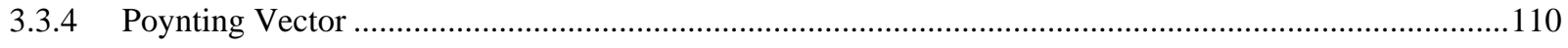

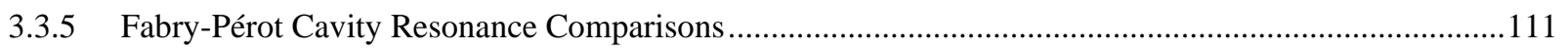

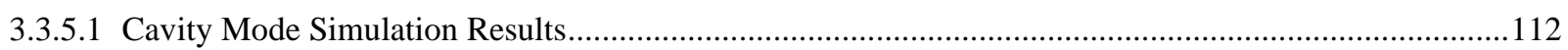

3.4 Plasmonic Lattices of Arrays of Gold Nanoparticles …....................................................................113

3.4.1 Parametric Sweeps of Critical Lattice Parameters ….........................................................................113

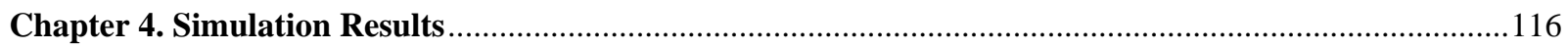

4.1 Initial Critical Lattice Parameter Optimizations................................................................................117

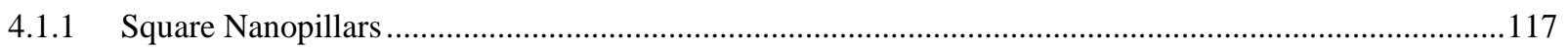

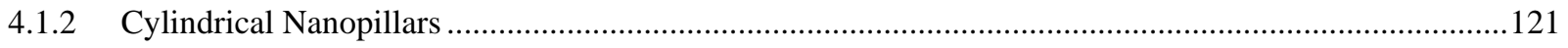

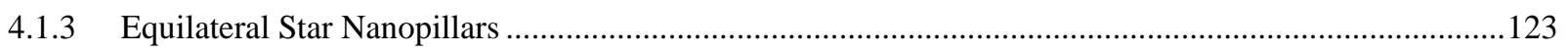

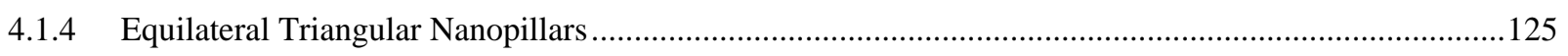

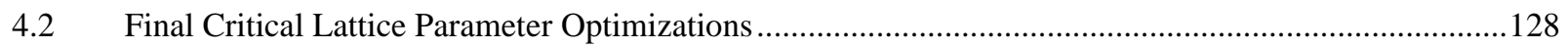

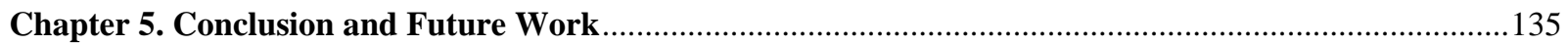

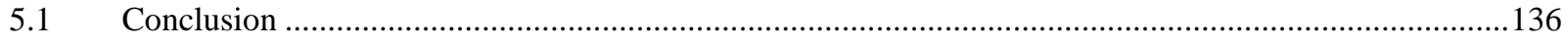

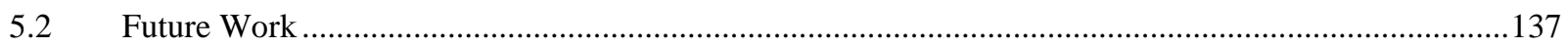

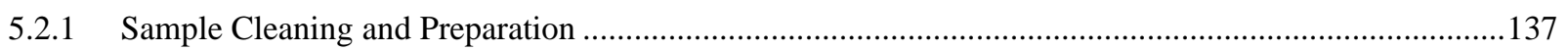

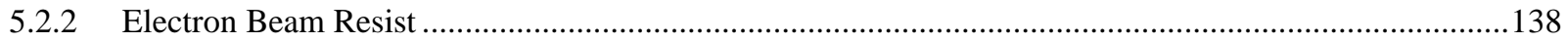

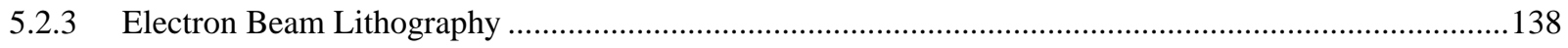

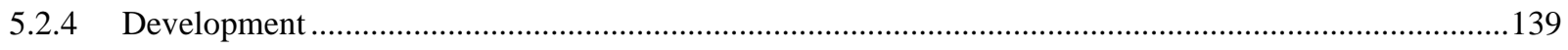

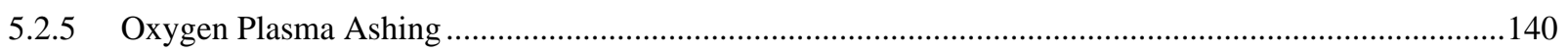

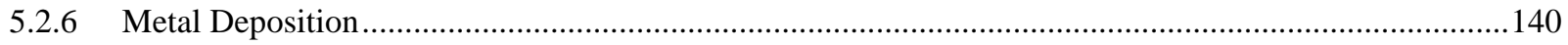




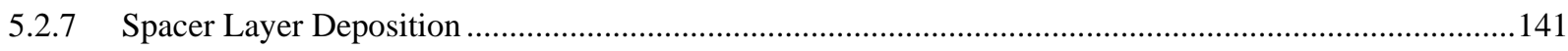

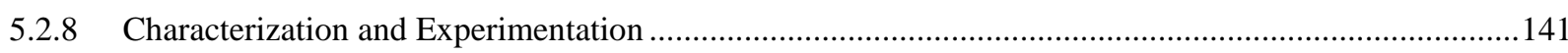

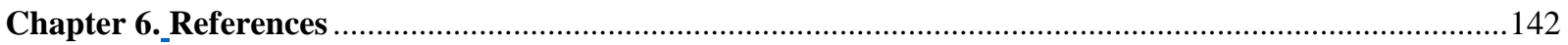

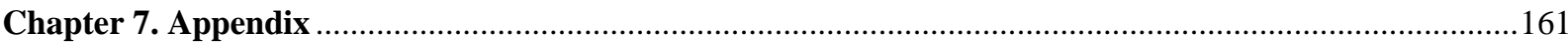

\section{List of Figures}

Figure 1.1 CCD image detection and processing schematic including analog signal processing, analog-to-digital converter (ADC), and digital image representation.........................................................................................

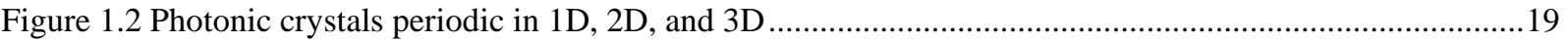

Figure 1.3 Process overview of fluorescent-labeled (F) flow system for integrated LOC biosensing technologies...23 Figure 1.4 Label-free sensor transducer showing immobilized antibodies on the substrate surface (red) and antigens (blue) depicting bonding between the two .24

Figure 1.5 SERS technique employing the use of gold NPs 27

Figure 1.6 Device schematic highlighting the functional regions for portable and rapid biosensing involving microfluidic flow channels. The entire sample is pumped in, the analyte DNA undergoes polymerase chain reaction (PCR) for amplification and is then separated from other biologicals present in the sample via hydrogel, the specific bonding regions of the analyte DNA are separated from the rest of the analyte DNA strand (i.e., electrophoresis), these separated bonding regions are then conjugated with fluorescing labels engineered to bind to these regions, then passed over a plasmonic lattice structure for fluorescence enhancement (adapted from [252]). An overview of the free flow process is also included showing the labeled sample passing over the localized enhancement regions

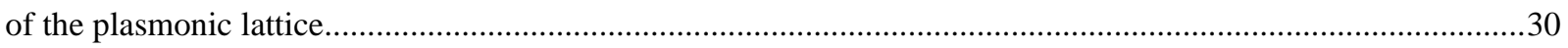

Figure 2.1 Kretschmann configuration for analyte analysis using SPR ...........................................................34

Figure 2.2 SPP wave propagation and field decay length over a given distance .................................................35

Figure 2.3 Confined longitudinal wave nature of the TM SPP modes ..............................................................36

Figure 2.4 Confined SPP wave vector and charge oscillation across a metal/dielectric interface in accordance with

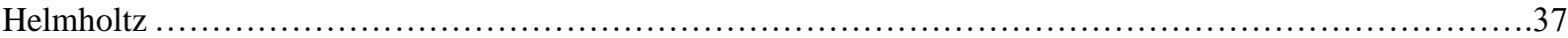

Figure 2.5 Regions of SPP resonance via irradiation for bulk materials, thin metal films, and spherical metal NP. .38

Figure 2.6 Metal NP electron cloud oscillation resulting from incident EM radiation. Accompanying radiative and non-radiative processes arising from an irradiated metal NP including heat dissipation, MEF, LSPR, SERS, and electron scattering.

Figure 2.7 Jablonski diagram representing A) the energy levels of an excited cadmium-selenium zinc/sulfide $(\mathrm{CdSe} / \mathrm{ZnS}) \mathrm{QD}$ (as used in experimentation) of this label (a TE dipole behaving as an oscillating point charge) inlaid, and B) the electronic and vibrational energy levels resulting from excitation of the single quantum emitter and the subsequent radiative and non-radiative decay processes .......................................................................50

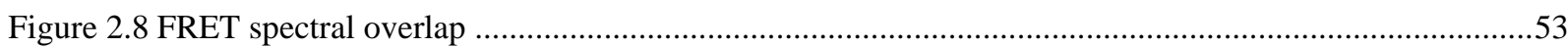


Figure 2.9 Types of fluorescence quenching.

Figure 2.10 Relationship between cavity mode resonance and the transition spectrum of the emitter in the case where the line width of the cavity mode is broader than the transition line width of the emitter.

Figure 2.11 Reverse Kretschmann device architectures employ attenuated total internal reflection (ATIR) methodologies showing polar $(\theta)$ and azimuthal $(\phi)$ angles. Confined fields SPP and LSP modes coupled with a nearby fluorophore at $\lambda_{e m}$. .64

Figure 2.12 Isolated nanosphere (NS) in the vicinity of an active InGaN QW is GaN

Figure 2.13 Two-step emission process and coupling rates: 1) energy transfer from the emitter into SP modes, and 2) the radiative coupling of those SP modes into radiation modes.

Figure 2.14 Angular distribution of SP-coupled emission resulting from SPPs and LRSPPs observed in the simulation of a reverse-Kretschmann system architecture comprised of an LSPR-supporting array of metal NPs

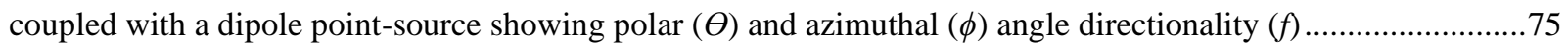

Figure 2.15 2D array of metal NPs atop the active QW region of a LED ..................................................76

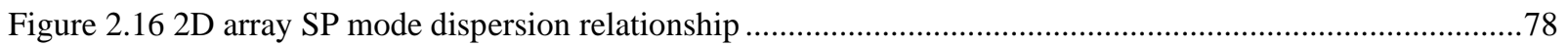

Figure 2.17 Excitation and emission spectra of a fluorophore including the correlation between the amplitude of photonic excitation and the intensity of photonic emission.....

Figure 2.18 Structure of a QD probe conjugated with a biomolecule including structural elements, roughly drawn to scale.

Figure 3.1 The Yee cell. Positions of the various field components shown with the $E$-field components located at the points of the cell with the $H$-field components located in the center of each face of the cell. ..95 Figure 3.2 Yu-Mittra method one. The presence of a PEC is accounted for by reducing the contour integral $C$ to include only the region outside the PEC where the $E$-field is non-zero.

Figure 3.3 Yu-Mittra method two. An effective permittivity is assigned to each permittivity component in the Yee cell weighted by the fraction of the mesh step inside material one or material two. If more than two materials are found in a single cell, the method reverts to Staircasing for that cell. .105

Figure 3.4 Start (left), End (right), and Full (center bottom) monitor apodization options in Lumerical FDTD ....111

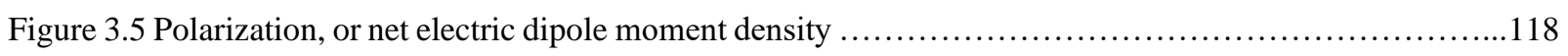

Figure 3.6 Magnetization, or net magnetic dipole moment density ..................................... 119

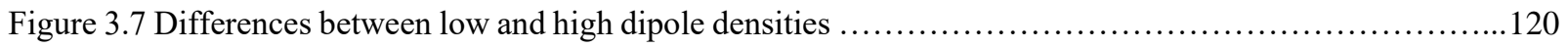

Figure 3.8 Cavity geometry. Fabry-Pérot interferometer simulation layout with dimensions similar to the cavity

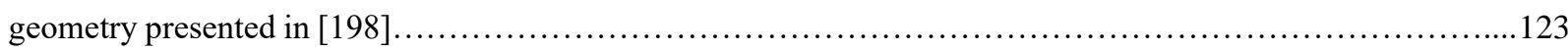

Figure 3.9 Resonance comparison of the Fabry-Pérot cavity mode for Al, Ag, Au, and PEC plates.................124

Figure 3.10 Recorded power on far side of cavity (opposite of the dipole source) as a function of dipole distance

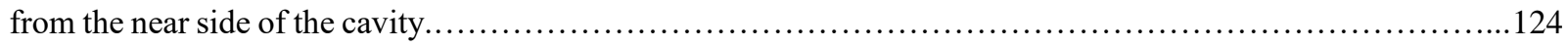

Figure 3.11 3D Perspective Simulation View of Initial Square Lattice Comprised of Square Pillars..............125

Figure 3.12 Cross-Sectional View of Initial Pillar Composition and Dimensions................................126 
Figure $4.1 E$ vs. $a$ vs. $\lambda$ parametric sweep data obtained from the point monitor showing peak $E$-Field occurred at $a$ $=270 \mathrm{~nm}$

Figure $4.2 E$ vs. $l$ vs. $\lambda$ parametric sweep data obtained from the point monitor showing peak $E$-Field occurred at $l$ $=50 \mathrm{~nm}$

Figure $4.3 E$ vs. $h$ vs. $\lambda$ parametric sweep data obtained from the point monitor. The data shows the peak $E$-Field occurred at $h=175 \mathrm{~nm}$ (70-nm thick Au Layer).

Figure 4.4 (Top Center) surface-enhanced $E$-field enhancement $\left(|E| /\left|E_{0}\right|\right)$ of 6 reproduced from [240] with a plane wave source emitting at $\lambda=565 \mathrm{~nm}$, (Bottom Left) surface-enhanced $E$-field enhancement $\left(|E| /\left|E_{0}\right|\right)$ of 61 of the parametrically optimized lattice with a plane wave source emitting at $\lambda=596.593 \mathrm{~nm}$, and (Bottom Right), surfaceenhanced $E$-field enhancement $\left(|E| /\left|E_{0}\right|\right)$ of 30 of the parametrically optimized lattice with a plane wave source emitting at $\lambda=565 \mathrm{~nm}$. The same dynamic range of 0 (dark blue) to 50 (red) was used for direct comparison.

.132

Figure $4.5 E$ vs. $a$ vs. $\lambda$ parametric sweep data obtained from the point monitor. The data shows the peak $E$-field occurred at $a=275 \mathrm{~nm}$

Figure 4.6 $E$ vs. $r$ vs. $\lambda$ parametric sweep data obtained from the point monitor. The data shows the peak $E$-field occurred at $r=35 \mathrm{~nm}$.

Figure $4.7 E$ vs. $h$ vs. $\lambda$ Parametric Sweep Data Obtained from the Point Monitor. The Data Shows the Peak $E$-field occurred at $h=205 \mathrm{~nm}$ (100-nm thick Au Layer)

Figure 4.8 Surface-enhanced $E$-field $\left(|E| /\left|E_{0}\right|\right)$ of the parametrically optimized lattice with a plane wave source emitting at $\lambda=599.7 \mathrm{~nm}$.

Figure $4.9 E$ vs. $a$ vs. $\lambda$ parametric sweep data obtained from the point monitor. The data shows the peak $E$-field occurred at $a=400 \mathrm{~nm}$.

Figure $4.10 E$ vs. $r$ vs. $\lambda$ parametric sweep data obtained from the point monitor. The data shows the peak $E$-field occurred at $r_{\text {center-to-vertices }}=45 \mathrm{~nm}, r_{\text {inner-points }}=22.5 \mathrm{~nm}$.

Figure $4.11 E$ vs. $h$ vs. $\lambda$ parametric sweep data obtained from the point monitor. The data shows the peak $E$-field occurred at $h=155 \mathrm{~nm}$ (50-nm thick Au Layer).

Figure 4.12 Surface-enhanced $E$-field $\left(|E| /\left|E_{0}\right|\right)$ of the parametrically optimized lattice with a plane wave source emitting at $\lambda=601.205 \mathrm{~nm}$.

Figure $4.13 E$ vs. $a$ vs. $\lambda$ parametric sweep data obtained from the point monitor. The data shows the peak $E$-field occurred at $a=265 \mathrm{~nm}$

Figure $4.14 E$ vs. $l$ vs. $\lambda$ parametric sweep data obtained from the point monitor. The data shows the peak $E$-field occurred at $l=50 \mathrm{~nm}$.

Figure $4.15 E$ vs. $h$ vs. $\lambda$ parametric sweep data obtained from the point monitor. The data shows the peak $E$-Field occurred at $h=165 \mathrm{~nm}$ (60-nm thick Au Layer).

Figure 4.16 Surface-enhanced $E$-field $\left(|E| /\left|E_{0}\right|\right)$ of the parametrically optimized lattice with a plane wave source emitting at $\lambda=598.202 \mathrm{~nm}$. 
Figure 4.17 XZ cross-sectional perspective of the lattice structure including nanopillar composition and labeled parameters simulated in FDTD.

Figure 4.18 Simulated $E$-field enhancement as a function of $\lambda, a, d$, and $t$ from parameter sweeps for the cylinder nanopillar geometry with $\mathrm{d} x, \mathrm{~d} y$, and $\mathrm{d} z=10 \mathrm{~nm}$. Highlighted in red is the maximum $E$-field enhancement factor of 18.27 found at $\lambda=744 \mathrm{~nm}, a=375 \mathrm{~nm}, d=50 \mathrm{~nm}$, and $t=5 \mathrm{~nm}$. Inlaid is the point monitor located at $t_{z \max }$ of the center nanopillar in the $3 \times 3$ array where the data was collected.

Figure 4.19 Simulated $E$-field enhancement as a function of $\lambda, a, l$, and $t$ from parameter sweeps for the square nanopillar geometry with $\mathrm{d} x, \mathrm{~d} y$, and $\mathrm{d} z=10 \mathrm{~nm}$. Highlighted in red is the maximum $E$-field enhancement factor of 22.87 found at $\lambda=588 \mathrm{~nm}, a=325 \mathrm{~nm}, l=50 \mathrm{~nm}$, and $t=80 \mathrm{~nm}$. Inlaid is the point monitor located at $t_{z \max }$ of the center nanopillar in the $3 \times 3$ array where the data was collected. Also included is the result found in [240] verified by these data.

Figure 4.20 Simulated $E$-field enhancement as a function of $\lambda, a, l$, and $t$ from parameter sweeps for the equilateral star nanopillar geometry with $\mathrm{d} x, \mathrm{~d} y$, and $\mathrm{d} z=10 \mathrm{~nm}$. Highlighted in red is the maximum $E$-field enhancement factor of 18.73 found at $\lambda=599 \mathrm{~nm}, a=350 \mathrm{~nm}, l=75 \mathrm{~nm}$, and $t=30 \mathrm{~nm}$. Inlaid is the point monitor located at tzmax of the center nanopillar in the $3 \times 3$ array where the data was collected.

Figure 4.21 Simulated $E$-field enhancement as a function of $\lambda, a, l$, and $t$ from parameter sweeps for the equilateral triangle nanopillar geometry with $\mathrm{d} x, \mathrm{~d} y$, and $\mathrm{d} z=10 \mathrm{~nm}$. Highlighted in red is the maximum $E$-field enhancement factor of 19.45 found at $\lambda=617 \mathrm{~nm}, a=275 \mathrm{~nm}, l=75 \mathrm{~nm}$, and $t=80 \mathrm{~nm}$. Inlaid is the point monitor located at tzmax of the center nanopillar in the $3 \times 3$ array where the data was collected.

Figure 4.22 FDTD planar monitor data $\left(|E||| E_{0} \mid\right)$ for all parametrically optimized lattice structures post-processed using MATLAB: (a) peak surface $E$-field enhancement factor of 51.98 for the lattice of cylindrical nanopillars at $\lambda$ $=701 \mathrm{~nm}$, (b) peak surface $E$-field enhancement factor of 335.39 for the lattice of equilateral star nanopillars at $\lambda=$ $625 \mathrm{~nm}$, (c) peak surface $E$-field enhancement factor of 211.10 for the lattice of square nanopillars at $\lambda=614 \mathrm{~nm}$, and (d) peak surface $E$-field enhancement factor of 251.52 for the lattice of equilateral triangle nanopillars at $\lambda=$ $648 \mathrm{~nm}$. The same dynamic range of 0 (dark brown) to 50 (white) was used in (a) through (d) for direct comparison.

Figure 5.1 Illustration of the proximity effect that occurs in EBL a) generation of secondary electrons b) interaction of the secondary electrons with the primary electrons c) graphic of the proximity effect in high-density patterning

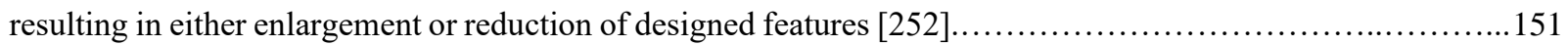

Figure 5.2 Overview of the HSQ- and non-HSQ-based electron beam lithography techniques [252] ............152

Figure 5.3 Process flow for plasmonic device fabrication using HSQ-based resist including metal and dielectric layer deposition [252]..... 153

Figure 5.4 Diagram of the characterization set up for testing of the plasmonic lattice (adapted from [252]). .153 


\section{List of Tables}

Table 2.1 Timescale ranges for the various processes in fluorescence.

Table 2.2 Select model analytes analyzed in PEF including analysis time, analyzed matrix, assay format, and

LOD. .56

Table 2.3 Select biomarkers analyzed in PEF biosensing including information on analysis time, analyzed matrix, assay format, and LOD

Table 2.4 Select pathogens and toxins analyzed in PEF biosensing including information on analysis time, analyzed matrix, assay format, and LOD.

Table 2.5 Comparison of respective field intensity enhancements, fabrication techniques, and resonance conditions of select SPP- and LSP-supporting metal nanostructures

Table 2.6 Experimental fluorescence enhancement factors of certain fluorescent labels used in conjunction with continuous metallic films, the intrinsic quantum yields of the fluorophores (where available), and the type of SP modes supported by these nanostructures, respectively

Table 2.7 Experimental fluorescence enhancement factors of certain fluorescent labels used in conjunction with chemically synthesized metallic NPs, the intrinsic quantum yields of the fluorophores (where available), and the type of SP modes supported by these nanostructures, respectively

Table 2.8 Experimental fluorescence enhancement factors of certain fluorescent labels used in conjunction with metallic NPs fabricated using lithography, the intrinsic quantum yields of the fluorophores (where available), and the type of SP modes supported by these nanostructures, respectively . .74

Table 2.9 Other commonly used fluorescent labels showing label name, excitation and emission peak wavelengths, and reported quantum yield

Table 2.10 Alexa fluorophores (fluor) available from ThermoFisher Scientific showing label name, excitation and emission peak wavelengths, reported quantum yield, lifetime of excited state, molar mass (MM), and extinction coefficient $(\varepsilon)$.

Table 2.11 Cadmium-selenide/zinc-sulfide ( $\mathrm{CdSe} / \mathrm{ZnS})$ core-shell QDs coated with octadecylamine (ODA) stabilizing ligand in toluene available from NNCrystal, US Corporation showing label product number, absorbance (excitation) and emission peaks, reported quantum yield, and concentration in solution. . .88

Table 2.12 Timeline of significant events in the history of FDTD. Continues ..................................8 89

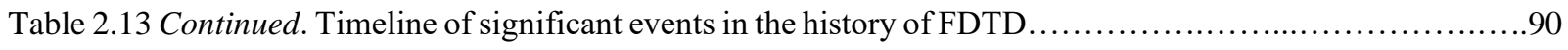

Table 2.14 Quantities and coefficients contained within Maxwell's equations ...............................90

Table 4.1 Parameter ranges swept for the square nanopillar geometry. .132

Table 4.2 Parameter ranges swept for the cylinder nanopillar geometry 134

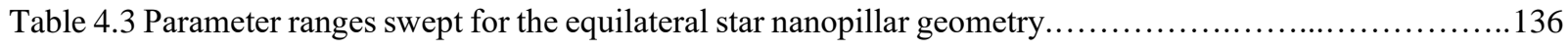

Table 4.4 Parameter ranges swept for the equilateral triangle nanopillar geometry.............................139

Table 4.5 Optimized surface $E$-Field enhancements observed for each pillar geometry.....

Table 4.6 Plasmonic lattice parameters swept. Shown in parentheses are the number of data sets recorded from each $a$ and $t$ sweep for each $l$. 
Table 4.7 Optimal parameters determined from the parametric analyses performed including $E$-field enhancement factors, $E$-field intensities, $\Delta \lambda$, and $\lambda$ where peak enhancement was observed for the four nanopillar feature geometries, respectively.

Table 4.8 Peak $E$-field enhancement factors, $E$-field intensities, $\Delta \lambda$, and $\lambda$ for the four optimized nanopillar feature geometries, respectively.

Table 7.1 QDs containing antibody or streptavidin conjugates available from ThermoFisher Scientific showing label name with absorbance (excitation) wavelength ranges and emission peaks

Table 7.2 QD cell labeling kits available from ThermoFisher Scientific showing label name with excitation wavelength ranges and emission peaks.

Table 7.3 Non-targeted fluorescent QDs available from ThermoFisher Scientific showing label name with excitation wavelength ranges and emission peaks for in vivo imaging of tumor and non-tumor vasculature in mice.

Table 7.4 Cadmium-selenide (CdSe) core QDs coated with octadecylamine (ODA) stabilizing ligand in toluene available from NNCrystal, US Corporation showing label product number, absorbance (excitation) and emission peaks, reported quantum yield, and concentration in solution

Table 7.5 Cadmium-selenide/zinc-sulfide (CdSe/ZnS) core-shell QDs coated with carboxylic acid stabilizing ligand in water available from NNCrystal, US Corporation showing label product number, absorbance (excitation) and emission peaks, reported quantum yield, and concentration in solultion

Table 7.6 Cadmium-sulfide (CdS) core QDs coated with oleic acid stabilizing ligand in toluene available from NNCrystal, US Corporation showing label product number, absorbance (excitation) and emission peaks, reported quantum yield, and concentration in solultion 174

Table 7.7 Cadmium-telluride (CdTe) core coated with octadecylphosphoric (ODPA) acid in toluene available from NNCrystal, US Corporation showing label product number, absorbance (excitation) and emission peaks, reported quantum yield, and concentration in solultion

Table 7.8 Cadmium-telluride/selenium-sulfide (CdTe/SeS) type-II core-shell QDs coated with octadecylamine (ODA) stabilizing ligand in toluene available from NNCrystal, US Corporation showing label product number, absorbance (excitation) and emission peaks, reported quantum yield, concentration in solultion, and full-width halfmaximum (FWHM) .

Table 7.9 Cadmium-selenide/zinc-sulfide (CdSe/ZnS) QD microspheres coated with carboxylic acid or streptavidin stabilizing ligands in water available from NNCrystal, US Corporation showing label product number, absorbance (excitation) and emission peaks, reported quantum yield, concentration in solultion, and full-width half-maximum (FWHM) .

Table 7.10 Lead-selenide ( $\mathrm{PbSe}$ ) core QDs coated with oleic acid stabilizing ligand in toluene available from NNCrystal, US Corporation showing label product number, absorbance (excitation) and emission peaks, reported quantum yield, concentration in solultion, and full-width half-maximum (FWHM) 
Table 7.11 Lead-sulfide $(\mathrm{PbS})$ core QDs coated with oleic acid stabilizing ligand in toluene available from NNCrystal, US Corporation showing label product number, absorbance (excitation) and emission peaks, reported quantum yield, concentration in solultion, and full-width half-maximum (FWHM) .

Table 7.12 Copper-indium-sulfide/zinc-sulfide (CuInS/ZnS) core-shell QDs coated with oleic acid/oleyamine stabilizing ligands in toluene available from NNCrystal, US Corporation showing label product number, absorbance (excitation) and emission peaks, reported quantum yield, concentration in solultion, and full-width half-maximum (FWHM).....

Table 7.13 Copper-indium-sulfide/zinc-sulfide (CuInS/ZnS) core-shell QDs coated with carboxylic acid stabilizing ligand in water available from NNCrystal, US Corporation showing label product number, absorbance (excitation) and emission peaks, reported quantum yield, concentration in solultion, and full-width half-maximum (FWHM) 175

Table 7.14 Indium-phosphide/zinc-sulfide (InP/ZnS) core-shell QDs coated with oleyamine stabilizing ligand in toluene available from NNCrystal, US Corporation showing label product number, absorbance (excitation) and emission peaks, reported quantum yield, concentration in solultion, and full-width half-maximum (FWHM) .176

Table 7.15 Indium-phosphide/zinc-sulfide (InP/ZnS) core-shell QDs coated with carboxylic acid stabilizing ligand in water available from NNCrystal, US Corporation showing label product number, absorbance (excitation) and emission peaks, reported quantum yield, concentration in solultion, and full-width half-maximum (FWHM) 176

Table 7.16 Manganese-doped zinc-selenide (Mn: ZnSe, or D-dots) QDs coated with oleyamine stabilizing ligand in toluene available from NNCrystal, US Corporation showing label product number, absorbance (excitation) and emission peaks, reported quantum yield, concentration in solultion, and full-width half-maximum (FWHM) 176

Table 7.17 Manganese-doped zinc-selenide (Mn: ZnSe, or D-dots) QDs coated with carboxylic acid stabilizing ligand in water available from NNCrystal, US Corporation showing label product number, absorbance (excitation) and emission peaks, reported quantum yield, concentration in solultion, and full-width half-maximum (FWHM) 


\section{Chapter 1}

\section{Introduction}

Chapter 1 provides an overview of current challenges facing biosensor development, details the basics of photonic crystals $(\mathrm{PhC})$, elaborates on optical biosensors differentiating between the label-free and labeled sensing modalities, outlines the principle of surface-enhanced Raman spectroscopy (SERS), explains what plasmonics is, why it is explored in the field of optical biosensors, and how it can be exploited to improve biosensor sensitivity and reduce their LOD. Finally, the goals of the research conducted in this thesis are explicitly stated. 


\subsection{Executive Summary}

\subsubsection{Problem Statement}

The biosensor market has grown significantly over the past two decades and is forecasted to exceed more than a $\$ 30$ billion market value by the year 2024 [1]. This incredible growth witnessed in the global biosensor market is driven by the following major factors: 1) significant technological advancements leading to the emergence of nanotechnology-based biosensors, 2) increased usage of biosensors to monitor biologicals such as glucose levels for diabetics, and 3) increased demand for home point-of-care (POC) handheld devices [1-2]. Conversely, the major factors restraining the global biosensor market are a slow commercialization rate, higher costs associated with the research and development, and a general reluctance to embrace newer treatment practices [1-2]. The global biosensor market can be segmented by application, type, product type, regional analysis, and product purchase incentive. Applications include POC, home diagnostics, research laboratories, biodefense, and environmental monitoring [2, 11]. Biosensors can be classified as either electrochemical, piezoelectric, optical, thermal, or nanomechanical that can be either wearable or non-wearable [2]. The high selectivity, sensitivity, and simplicity of monitoring fluorescence emission (i.e., fluorescence spectroscopy) for the detection of a target molecule(s) lends to its usefulness as a metric in integrated lab-on-chip (LOC) biomedical microdevices $[11,40,46,53,57-58,60,86,98,105,160]$. Despite its advantages, fluorescence spectroscopy suffers multiple limitations such as low-intensity emission, relatively high sample background signal, system dark current (i.e., transducer noise), and low photo-stability [4-10, 1213, 17-18]. While there are multiple non-radiative factors that adversely affect the fluorescence emission process (e.g., internal conversion, intersystem crossing, quenching, etc.), optimizing the excitation rate of an isotropic ensemble of emitters can directly result in an increased emission rate of those emitters [4-10, 12-13, 17-18]. The centerpiece of plasmon-enhanced biosensing is to boost and control the spontaneous emission rate of fluorophore(s) utilized in the detection of specific pathogens (e.g., Aspergillus fumigatus, Burkholderia mallei, Burkholderia pseudomallei, cancer biomarkers, toxins, viruses, etc.) via the combination of fluorescent probes with noble metal nanostructures [158-174]. 
The resonant interaction of strong fluorescent emitters with plasmonic materials, known as plasmon-enhanced fluorescence (PEF), is a powerful mechanism driving improvements in fluorescence biosensing methodologies [8-9, 42, 66, 91, 143, 149, 164, 171]. PEF offers a means of achieving fluorescent emission enhancement coupled with decreased label lifetimes, therefore providing viability for emitters of lower quantum efficiency (QE) [15, 18, 72, 114, 125, 127]. Controlling spontaneous fluorescence emission provides a method of reducing the LOD by maximizing the emission rate of these labels near metal nanostructures, consequently granting real-time analyte detection capabilities [7-9, 40, 60, 99, 156-174].

\subsubsection{Research Goals}

The goal of the Dawson research group at West Virginia University is to design nanophotonic and nanoplasmonic lattice structures engineered to boost fluorescence emission and subsequent detection through the development of cost-effective and efficient LOC system architectures for real-time identification of labeled biological and chemical specimens. The quick-time diagnosis of various diseases allows for earlier detection of a wide variety of target analytes including cancer biomarkers and many infectious pathogens leading to a decrease in the mortality rates associated with these diseases $[11,25,158-174]$. The goal of the research effort presented in this thesis is to improve the fluorescence enhancement of fluorophores used as analyte labels in fluorescence spectroscopy by exploiting the LSPR of periodic gold nanoparticle (NP) arrays. The metallurgy of noble metals such as gold have been the topic of much research regarding fluorescence enhancement because of the ability of these metals to control and modify optical fields at the micro- to nano-scale via surface plasmons, or collective oscillations of electrons and charge density on and around a noble metallic surface via optical excitation [3-11, 13-19, 37-62, 73]. By exploiting and optimizing the plasmonic response, one can enhance the emission rate of these fluorescent probes by significantly enhancing their excitation rate, consequently reducing the LOD (i.e., the more photons being emitted per unit time, the higher the probability that a photodetector can detect the presence of an analyte in a biological sample). A fluorophore in proximity to a gold NP has been shown to directly influence the emission intensity of the fluorophore due to the strong plasmonic field induced by the irradiated NP [3-18, 22, 41-43, 46-56, 59-103]. 
This plasmonic field is controllable via selective clustering, functionalization, and sizing of the gold NPs with a plethora of biomedical applications such as bio-imaging, environmental monitoring, genomics, and faster medical diagnoses [11, 158-174]. The obtainable fluorescence emission in the presence of these gold NPs can be up to hundreds of times greater than that in the absence of gold NPs and is directly related to an overlap between the plasmonic resonance of gold NPs and the fluorescence emission wavelength [3-18].

Despite the multiple advantages offered within the scope of fluorescence spectroscopy, plasmonics faces a handful of important challenges. One of the main challenges confronting SPbased nanophotonics is the loss-confinement tradeoff arising from the short propagation length of the tightly confined SP modes [14, 48, 59-71]. This characteristic coupled with the fact that all electro-, magneto-optical, thermo-, etc. are inherently weak material effects prove problematic in the realization of compact active, efficient, and low-loss SP-based optical components. This disadvantage complicates the design of a plasmonic circuit or lattice that can be easily integrated into several active and passive devices [79-102]. Furthermore, despite the wide usage of SPcapable metals like gold and silver, these noble metals exhibit low melting points complicating their usage in CMOS (complementary metal-oxide semiconductors) and other conventional plasmonic applications $[29,88,118]$. Another detriment of plasmonics lies in the challenge of spatially resolving the extreme nm-scale field changes [61-65]. Consequently, more sophisticated optical characterization methods will need to be developed to resolve nm-scale plasmon resonances associated with the strong confinement and variation of these plasmon-enhanced fields [3-105]. Additionally, due to the confined nature of LSPR, a fluorophore or other quantum emitter must be present in the enhanced $E$-field occupying a very small effective volume for excitation and emission enhancement to occur $[42,61-65,74]$. 
The tasks associated with this research effort are as follows:

- Determine the optimal plasmonic lattice parameters for a large-area $(\sim 30 \mu \mathrm{m}$ x $30 \mu \mathrm{m})$ square lattice containing an array of multiple feature geometries engineered to yield peak $E$-field enhancement using finite difference time domain (FDTD) software

- Compare the resulting $E$-field enhancement factors found from the simulations at the resonant conditions of each feature geometry, respectively

- Based on the optimization results, determine which commercially available fluorescent label(s) (where applicable) possess an emission spectrum that overlaps with, or whose peak lies within, the resonance condition of each optimized lattice geometry, respectively

- Relate the simulated $E$-field enhancement factors to fluorescence emission to calculate theoretical fluorescence emission enhancement using the reported quantum efficiencies (if available) of the selected fluorophore(s)

If successful, this work will provide a systematic means of evaluating a multivariable parameter space to aid in the construction of plasmonic lattice structures engineered for improved fluorescence spectroscopy. This will be realized by exploiting the LSPR of an array of gold nanostructures to enhance the excitation and subsequent emission rate of fluorescent probes employed in PEF.

\subsection{Optical Transduction for Biosensing Applications}

The fundamental principle governing optical transduction involves taking energy from one form and transferring it into another, quantifying that energy change/input, and measuring the optical response [21, 120-121]. This methodology can be specifically tailored for plasmonic affinity biosensors incorporating infrared (IR) or ultraviolet (UV) electromagnetic (EM) energy with analyte-bioreceptor interactions [7, 9, 40, 99, 156-174]. Optical transduction techniques either directly, or indirectly, probe analyte(s) of interest using a photodetector to quantify the optical response typically performed by comparing some optical characteristic of the reflected radiation from the sample to that of the source beam (e.g., amplitude, and/or phase information) [21, 23-29]. Optical transducers can be used in conjunction with a radiative probe, or to directly measure the EM interaction between the sample and source. 
An interesting example of optical sensing where functionalized nanostructures plays a role is in surface-enhanced Raman spectroscopy (SERS) [23-29, 51, 69-71, 97, 99, 126]. In SERS, the Raman signal generated from a sample can be greatly enhanced when the sample itself is in proximity (nanoscale) to a flat or NP-patterned surface. This technique has been used within the field of biosensing by utilizing gold NPs as the SERS substrate and conjugating analyte(s) of interest to the surface(s) of the NP(s) [23-29, 69-71]. SERS will be later explored in Section 1.5. A common detector for this optical sensing modality is the charge-coupled device (CCD). CCDs are planar arrays of metal-oxide semiconductor capacitors (MOSCAPs) forming integrated circuits (ICs) etched onto a surface, typically silicon ( $\mathrm{Si}$ ), creating light-sensitive elements known as pixels. Incident photons generate charge packets on the CCD IC that can be electronically read and then transcribed into a digital copy of the light on the serial device [29, 120-121]. CCDs come in a variety of sizes and types with applications in areas like astronomy, biomedical detection and imaging systems, cell phone cameras, dental X-rays, mammography, and security surveillance camera technology [29, 120-121]. Figure 1.1 below visualizes the image detection and processing of a general CCD.

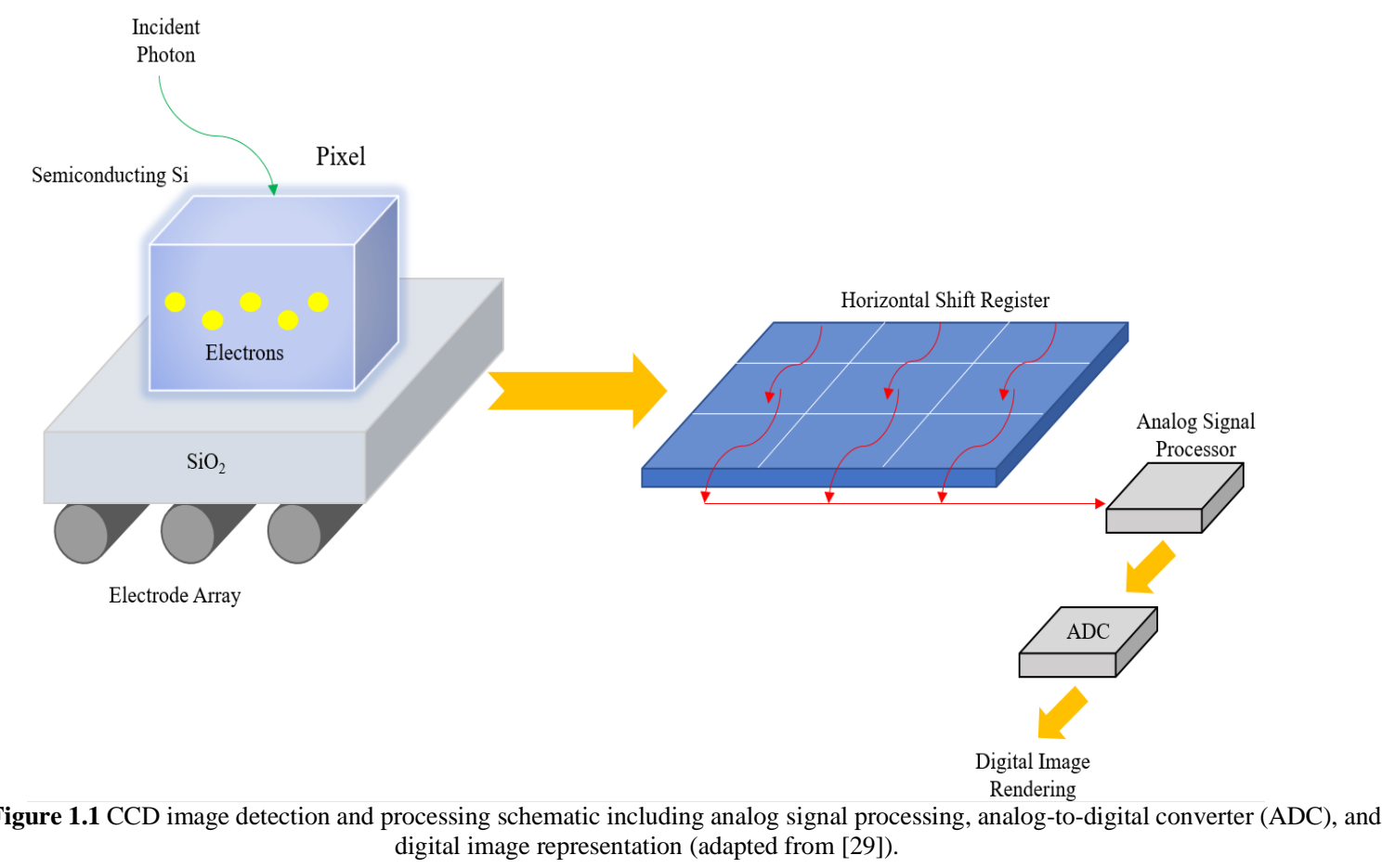




\subsection{Photonic Crystals}

Nanophotonic devices such as PhCs, diffracted Bragg reflectors (DBRs), fiber optics, and resonance cavities allow for the control of directionality, propagation, transmission, and other optical properties of incident light [17, 19, 31-36]. Photonic crystals (PhCs) are dielectric structures designed with periodically varying geometric constraints in one, two, or three dimensions (Figure 1.2) engineered to control the propagation of light at specific wavelengths [17, 19, 31-36]. In conjunction with a periodic variation in dielectric constant, photonic crystals also exhibit a periodic modulation in refractive index $(n)$.

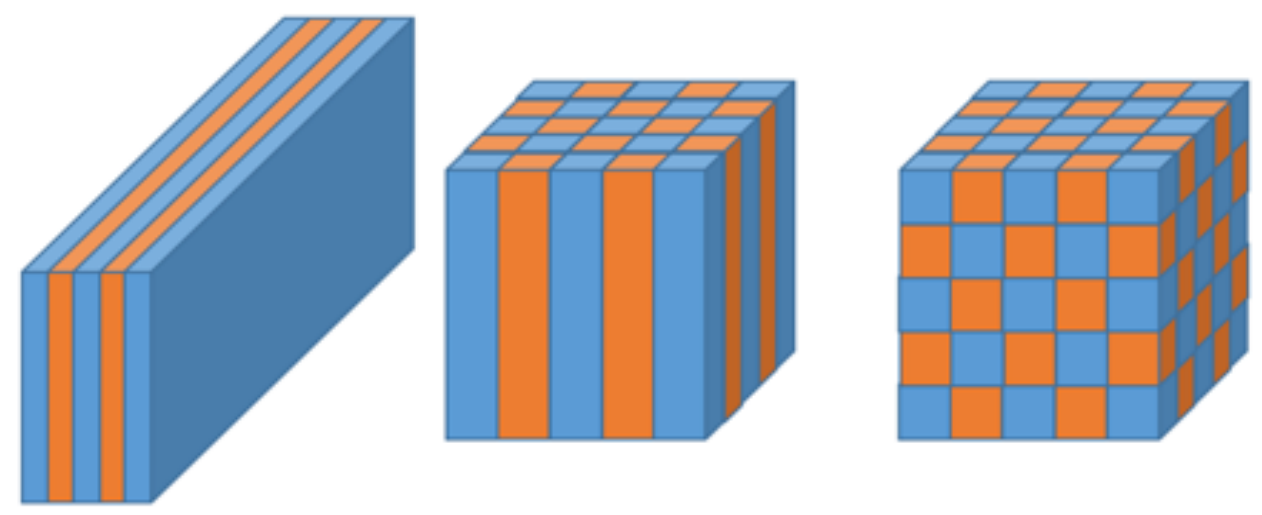

Figure 1.2 Photonic crystals periodic in 1D (left), 2D (center), and 3D (right) [36].

If the length scale of the modulation is at the same length scale as the wavelength of light with a sufficiently large index contrast, a photonic band gap (PBG) is created prohibiting the propagation of light at that wavelength. More specifically, the bandgap of a photonic crystal is directly related to the size and spacing between the structures comprising the crystal structure $[19,31-36,186]$. Introducing defects into the PhC lattice creates tunable cavity modes $[30-36,186]$. PhCs can be configured for use in both active and passive optical systems. In active configurations, the parameters of the PhC can be dynamically varied (e.g., feature length, hole depth, pillar height, slab thickness, etc.) and, consequently, alter the optical properties of the crystal [19, 30-36, 186].

Conversely in a passive configuration, these feature and lattice parameters are fixed, specifically engineered to either permit or prohibit certain wavelengths of light dependent upon the application $[19,34-36,186]$. These photonic lattice structures can be observed in nature in the structural coloring of various species of beetles, butterflies, some sea animals, and in peacock feathers $[19,33]$. 
PhCs have proven useful for a variety of applications such as spontaneous emission control, LED frequency tuning, solar cells, and LOC biosensing technologies. [17, 30-36, 105, 186]. In LOC fluorescent biosensors, the photonic bandgap of the $\mathrm{PhC}$ can be engineered for the spatial confinement or redirection of photons designed to enhance the $E$-field amplitude/intensity via resonant coupling (i.e., enhanced photon extraction vs. enhanced excitation, respectively) [30-36, 74-75, 186]. Fluorescence biosensing systems can utilize one or both of the methodologies mentioned above to enhance the fluorescence emission rate granting enhancement capabilities of up to three orders of magnitude [17-18, 34, 47, 186]. A notable drawback of using passive PhCs for fluorescence enhancement lies in the fact that enhancement relies on the interaction between the emitted photons and the crystal structure itself $[30-36,186]$. This is an important issue to consider since the interaction volume of analyte(s) in solution is quite small for passive $\mathrm{PhC}$ configurations. The use of an active PhC lattice lies beyond the scope of this work.

The goal of this thesis is to optimize fluorescence enhancement by incorporating a biocompatible, plasmon-capable metal like gold onto an engineered lattice of sub-micron dielectric pillars, much like a passive $\mathrm{PhC}$ device, for decreasing the LOD of visible wavelength fluorescence biosensors. The construction of this optimized plasmonic lattice will overcome the main drawback facing PhCs regarding fluorescence enhancement via localized regions of enhanced $E$-field in conjunction with a larger interaction volume. The uniform enhancement regions of a plasmonic lattice provide a larger operational area whereby the excitation enhancement rate of an ensemble of emitters can be enhanced.

\subsection{Optical Biosensors}

Biosensors capable of detecting disease-specific biomarkers are paramount across the field of healthcare ranging from initial detection and subsequent diagnosis to providing optimal therapy and treatment options [39-46, 53-58]. Such biosensors must be capable of specifically quantifying biologicals with higher temporal resolution. Additionally, these biosensors must be capable of monitoring the evolution of a target analyte [53-66]. Accounting for these multi-faceted operational requirements can be quite challenging considering the large number of different biomolecules present in a clinical sample while attempting to detect a single, or a small ensemble of, an analyte(s) of interest [59-66, 129-173]. 
The development of personalized medical care will demand highly selective, sensitive, and multiplexed platforms capable of meeting the above requirements. Highly sensitive biosensors are crucial since biochemical analytes like DNA, RNA, protein chains, single proteins, viral capsids, etc. are often only present in incredibly small concentrations (i.e., pg/mL to fg/mL) [40, 53-66, 156-173, 178]. The microarray offers a powerful option for detecting miniscule amounts of biological sample volumes [40].

Microarrays are incredibly useful for predictive and early disease diagnosis technologies [40]. In each microarray, thousands of reaction spots are functionalized over large areas typically spanning at least a few square centimeters (i.e., a biochip). It is a remarkably versatile and useful platform, particularly when dealing with very limited sample volumes in areas like cellomics, genomics, glycomics, metabolomics, proteomics, and transcriptomics [40, 157, 172, 183]. More sophisticated experimentation is possible by non-specialized, less technically endowed users via automated signal readouts, condition controlling systems, and partially automated dispensing systems [40]. Protein microarrays are primarily engineered to perform two main functions: 1) identify and quantify protein abundance, which is applicable across a range of cancer studies namely biomarker screening and searching and in the diagnosis of particular cancers via distinctive biological fingerprints, and 2) study biomolecular and protein functions involving the intermolecular interactions that reveal new molecular insight and/or important characteristics such as binding parameters, enzymatic functions and novel biomolecular interactions [40, 53-66, 156173]. Consequently, numerous specifically tailored protein microarrays have been researched.

Promising modalities of protein analysis span across a wide range of microarray device technologies including DNA/RNA detection, whole protein detection (i.e., antibody/antigen and whole-proteomes), and peptide detection (i.e., $\alpha$-helical peptides, $\beta$-turn peptides, sugar-modified peptides, and protein sequence peptides [9, 40, 47, 156-173]). Protein microarrays are supported by three (3) key functional criteria: 1) production of functionalized 'receptor' molecules, 2) surface chemistry, and 3) the development of high-throughput detection methods [40, 156-173]. These systems must often be integrated to selectively detect the activity and/or presence of specific proteins [40]. Microarrays designed for protein detection can be performed with or without the use of any label. Section 1.4.1 details labeled and label-free techniques in transducing nano-biological events. 


\subsubsection{Labeled Optical Biosensors}

Labels used within the realm of biodetection are defined as any molecule foreign to the experimental environment chemically or temporarily conjugated to an analyte of interest for detecting the activity or presence of a molecule $[127,174]$. Labeled detection methods require a preparation step usually with labels of lower quantum yield, sample synthesis, and purification [37-50]. Fluorescent and NP labeling typically involves the covalent bonding of the species via chemical coupling. For labeled modalities, fluorescent biosensors can take advantage of the plasmonic properties of thin metal films, single metal NPs, and metal NP arrays [81-96, 156-173]. Labeled biosensors have emerged as an alternative method for detection of DNA enabling lower cost and higher sensitivity [37-50, 156-173]. Fluorescent label detection methodologies are the most common and convenient modalities for transducing molecular events [4-12, 17-18, 42, 62$66,110-111,127,131-136]$. These probes come in many forms including fluorescent proteins, quantum dots (QDs), and small organic molecules [116, 118, 174, 182-183, 185]. Moreover, these probes possess a wide range of quantum yields and other material properties capable of meeting multiple application requirements and sensor limitations [11, 37-49]. Specifically, fluorescent probes offer a plethora of advantages in optical biosensing due to their chemical stability, easy manipulability, high sensitivity, and excitation and emission maxima that reside in the visible to IR regime of the EM spectrum [4-10, 23, 42-44, 174]. Other single quantum emitters, such as fluorescent semiconductor QDs and fluorescent carbon dots, are also capable of coupling to plasmonic nanostructures and can also be incorporated into labeled biodetection methods [127, 174, 182-183].

The demand for compact, low costing, and simple devices that can effectively detect very low (nanomolar (nM) to femtomolar (fM)) concentrations of biologicals in a sample has increased in recent years [37, 41-42, 57, 80, 91, 99]. More advanced fluorescence techniques like biomolecular fluorescence complementation (BFC), fluorescence correlation spectroscopy (FCS), and fluorescent resonance energy transfer (FRET) allow for the analysis of samples as small as 1 femtoliter (fL); a significant reduction of the LOD [99, 161-166]. Detecting trace amounts of an analyte is possible via the employment of specific fluorescing probes designed to bind with the target analyte. Labeled methods use a secondary receptor molecule designed to bind to the analyte of interest, altering the natural state of the biological material, and possibly its molecular behavior [4-10, 23, 41-44, 57, 80, 91, 161-166, 174]. 
Techniques requiring the unaltered natural state of a target molecule are not practical for label-based methods. Labeled biosensing methods do have certain advantages over their label-free counterparts through their ease of use, high sensitivity, lower cost, and dual-confirmation (i.e., analyte-fluorophore conjugation and subsequent fluorescence emission) leading to their implementation into applications like DNA/RNA detection and DNA electrophoresis [47, 50, 156, 158]. Figure 1.3 depicts the general layout and process flow of labeled fluorescence flow system for LOC biosensing. The research effort presented herein pursues plasmon-capable metal-coated lattice features for integrated LOC systems used in labeled fluorescence detection methods.

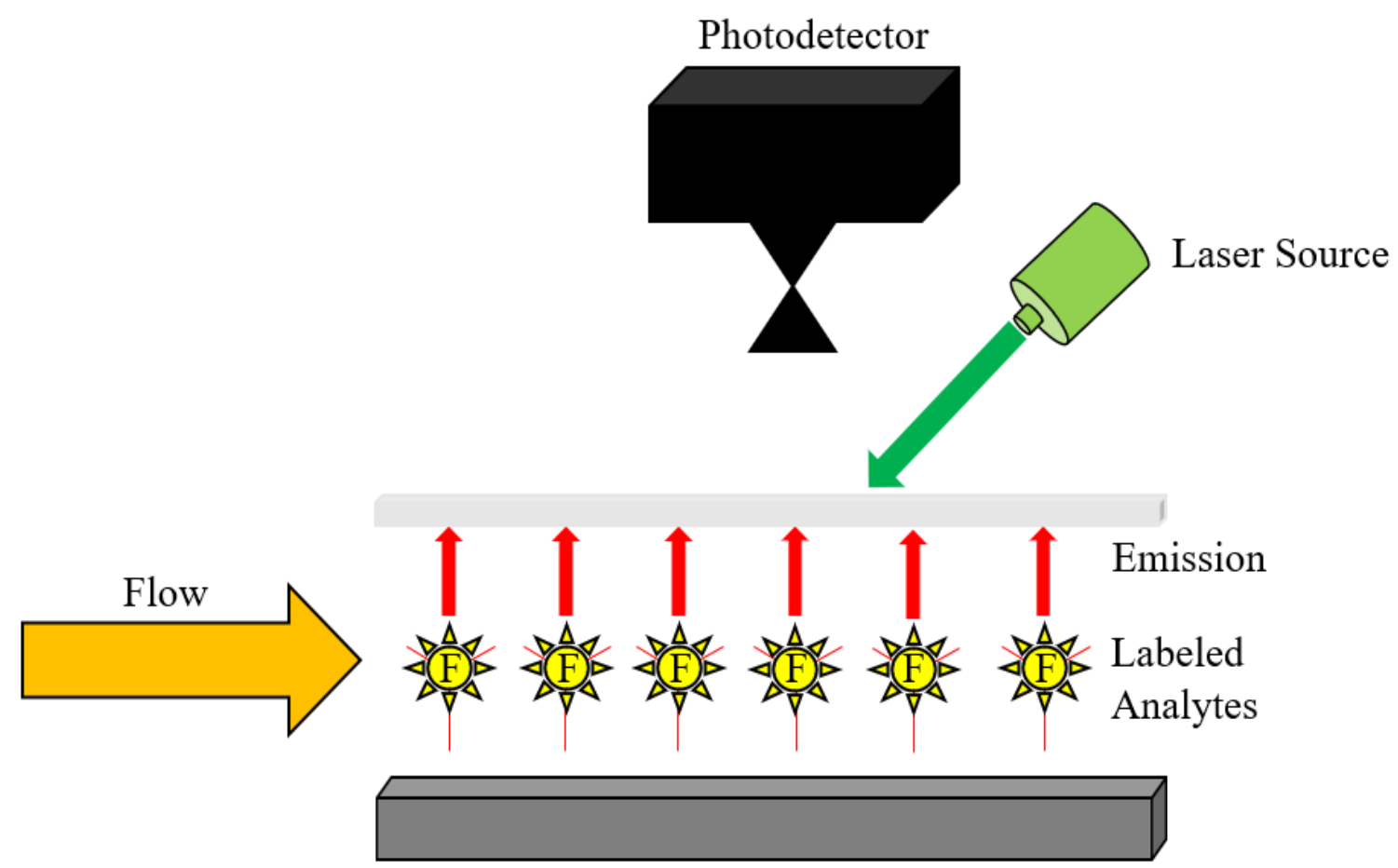

Figure 1.3 Process overview of fluorescent-labeled (F) flow system for integrated LOC biosensing technologies (adapted from [37], [40-41]). 


\subsubsection{Label-Free Optical Biosensors}

Label-free detection methods exploit the biophysical properties of a molecule such as molecular charge, molecular weight, or refractive index to determine the presence and/or activity of an analyte of interest [40, 53, 57-60]. Examples of label-free detection are anomalous reflections of gold surfaces, LSPR, mass spectrometry, microbalance, microcantilevers, quartz-crystal microbalance, and surface plasmon resonance (SPR) [59, 61-68, 70]. As previously elaborated, these methods can be used to detect and track molecular events in real-time allowing for a quicker time to diagnosis and treatment. LSPR and SPR can be used as a reference for label-free biosensing where the shift in the resonance peak delineates the presence of molecules. More specifically, this red-shift in the transduced signal is due to a change in the refractive index of the surface and is indicative of molecular presence (i.e., SERS) [23-28, 51, 69, 71, 97-99]. A label-free sensor commonly involves the immobilization of antibodies on the substrate surface creating functional capturing agents for antigens [37-44, 57-60]. Figure 1.4 delineates the previously mentioned labelfree device design.

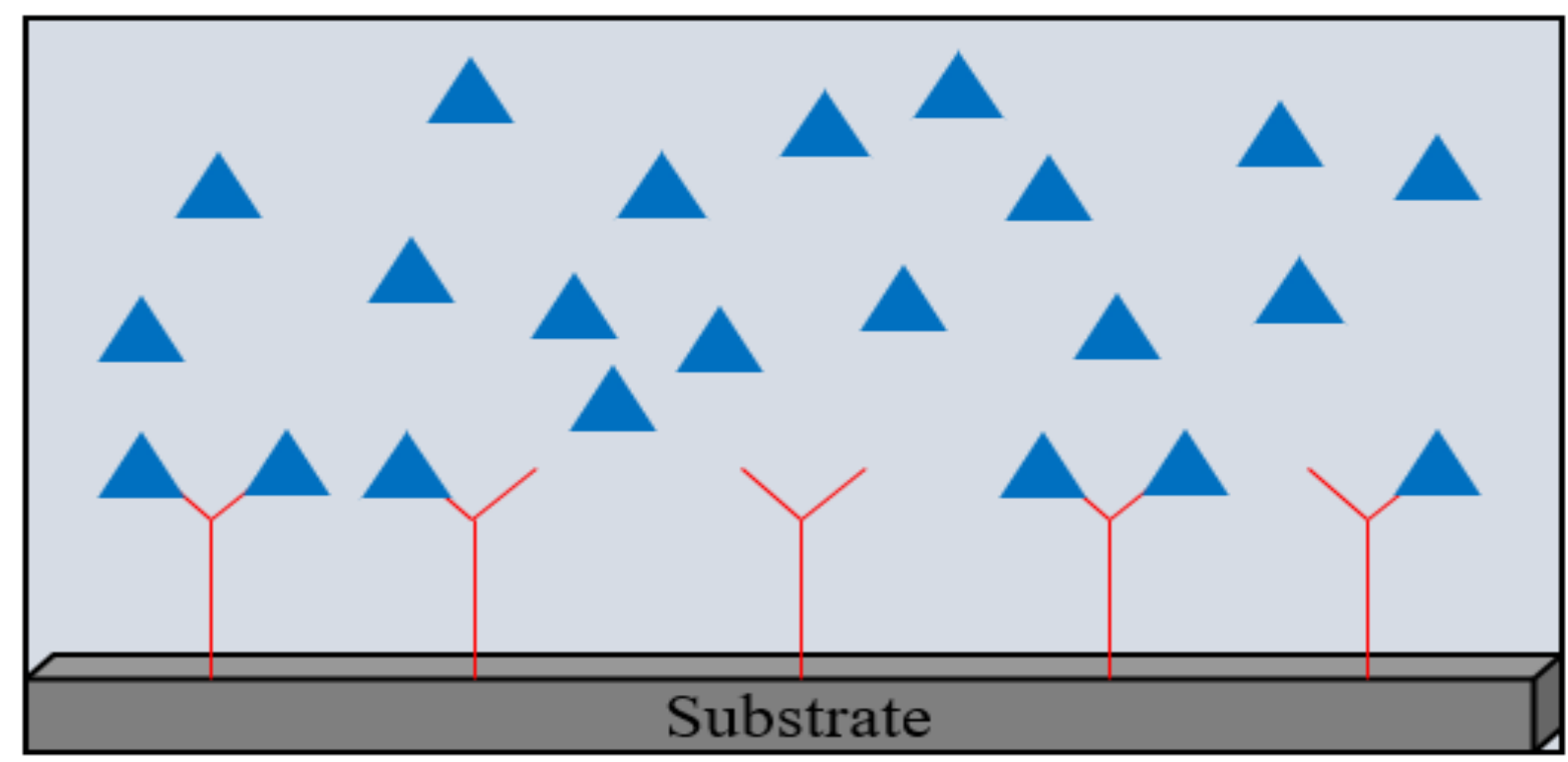

Figure 1.4 Label-free sensor transducer showing immobilized antibodies on the substrate surface (red) and antigens (blue) depicting some bonding between the two (adapted from [40], and [57-58]). 
Intermolecular interactions are typically transduced electrically, mechanically, or optically and are unencumbered by the use of any application-specific label probes; an advantage offered by label-free methods [40, 53, 57-60]. Label-free detection schemes also enable continuous monitoring of the affinity reaction, provide quantitative information on binding affinity and kinetics, and remove the need for multiple washing stages between assay steps that can complicate sample preparation requirements [40]. Direct and specific detection of biological analytes regarding their physical properties like charge, mass, or physical size pose multiple challenges in the development of a selective and sensitive label-free biosensor [57-60]. As a result, most biochemical assays exploit the high affinity of a receptor molecule to bond with a specific biomarker. In the case of protein detection, this receptor molecule is typically an antibody raised against the specific protein antigen while a complementary single stranded DNA is used for specific DNA detection [9, 40, 47, 156]. Following molecular bonding to, the receptor molecule can then be functionalized with a label that is easily measurable and quantifiable.

\subsection{Surface-Enhanced Raman Spectroscopy}

SERS is an optical detection technique that offers several analytical, biological, and engineering advantages over other traditional detection schemes [23-28, 51, 69, 71, 97, 99, 126]. The advantages characteristic of SERS includes an extremely high level of sensitivity, inherent molecular specificity for label-free targets, and sharp spectral bands [23-28]. In SERS, the target analyte is placed in proximity to a sub-micron or nanoscale metallic surface (e.g., aluminum, copper, gold, platinum, or silver) possessing nanoscopically defined features smaller than the wavelength of incident light [3-10, 47-55]. This incident monochromatic light leads to a surface plasmon mode which enhances EM energy surrounding the target molecule and results in a significant enhancement of inelastically scattered light (i.e., the Raman signal). This Raman signal is directly associated with the excitation of the localized surface plasmons (LSPs). SERS can provide resolution below the diffraction limit, with a spatial resolution defined by the wavelength

of the laser and the numerical aperture of the microscope lens objective $\left(r_{S}=\frac{0.61 \lambda_{\text {laser }}}{N A}\right)$ [23-25]. The inelastic scattering of light is due to a change in the wavelength reflected from the sample/substrate indicative of the discrete vibrational modes of polarizable molecules allowing for qualitative biochemical measurements [23-28, 51]. 
Weak scattering pertains to the polarizability of a molecule and is determined by the electron cloud of that molecule interacting with an $E$-field (i.e., a softer molecule like benzene more strongly scatters light than harder molecules like water) [25-27]. In short, LSPR greatly contributes to SERS, resultant of both chemical and EM mechanisms.

Enhanced EM fields and photon scattering result from electron oscillations in the conduction band within a metal surface [5-39]. The chemical mechanism of SERS is not as easily understood as the EM response, but is attributed to the intermediate state charge transfer manifesting from strong electron coupling between the analyte and the metal surface [5-28]. Both mechanisms have been demonstrated experimentally, and both play important roles in SERS [2528]. Despite chemical and EM contributions, it is believed that the EM enhancement is the greater of the two governing forces on the arousal of SERS [25-28, 47-55]. The SERS technique aims to increase the weak Raman signal intensity associated with the spontaneous scattering of light. Generally, there are two means through which SERS detection can be realized: 1) homogenously, where the target analyte is conjugated or absorbed onto metal NPs in solution (i.e., Raman enhancers), and 2) heterogeneously, where the targets in solution directly interact with SERS active sites at the particle(s) surface(s) [27-28]. Both homogeneous and heterogeneous reactions share the same advantages of ease of implementation, fast reaction rates, as well as uniform and repeatable SERS enhancement since NP synthesis is a highly uniform process [40-46].

An obvious disadvantage of the homogenous SERS reaction is, relatively speaking, low detection sensitivity since the Raman enhancers are dispersed in solution. However, this relatively low sensitivity can be circumvented via the use of enhanced microscopy techniques [40-46]. While heterogenous SERS reactions do generally enable greater sensitivity than homogenous SERS reactions, the time of analysis is typically longer, and chip fabrication is often more complicated [40]. SERS architectures can be engineered in a plethora of ways dependent on experimental needs, championing its versatility in optical transduction [21, 57, 120-121]. Figure 1.5 visualizes the SERS technique. In this work, periodic arrays of various gold NP geometries (i.e., SERS substrate) were modeled in FDTD software for determining the optimal lattice parameters yielding the peak, LSPR-enhanced $E$-field for reducing the LOD inherent of labeled fluorescence spectroscopy. 


\section{SERS Technique}

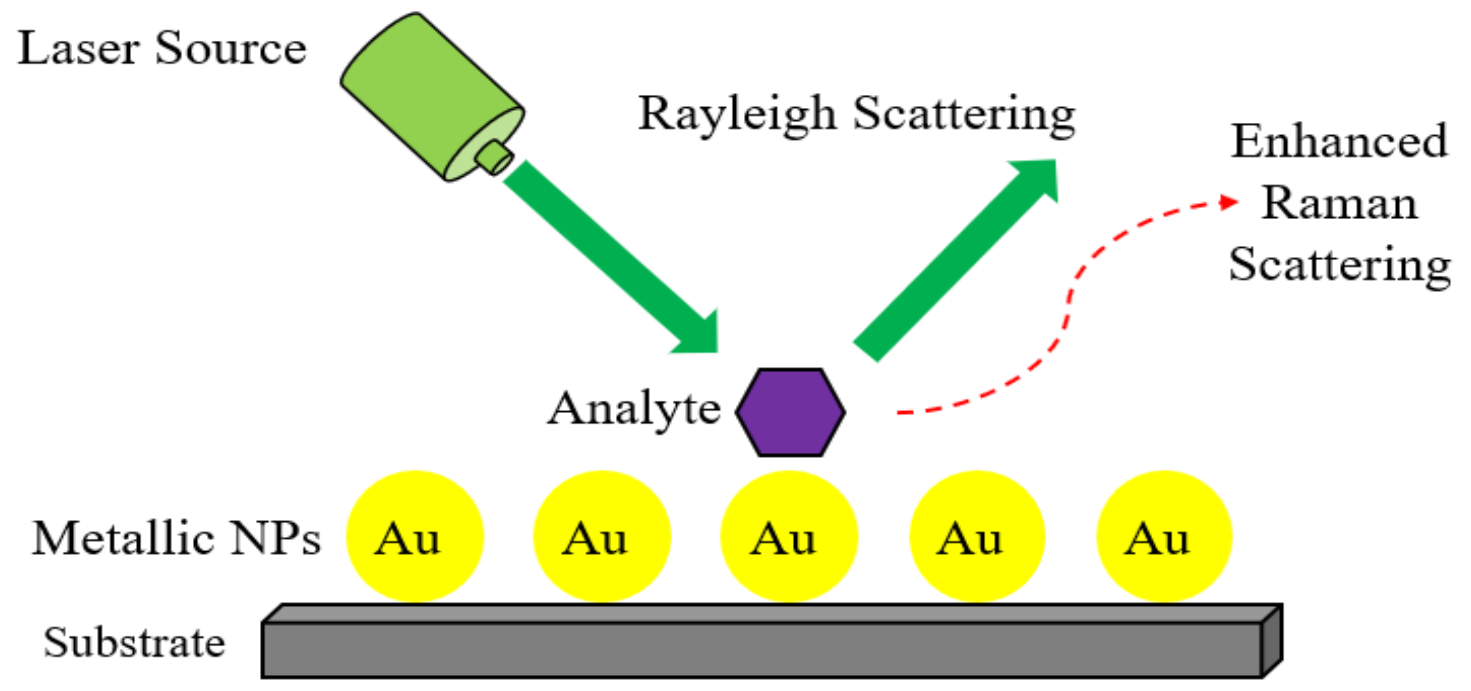

Figure 1.5 SERS technique employing the use of gold NPs (adapted from [25-28]).

\subsection{Fundamentals of Plasmonics}

The field of plasmonics explores the interaction between light and metal surfaces, and the corresponding oscillation of the electron density wave produced from this excitation [3-16, 41-43, 47-53, 55-56, 61-70, 72-73, 75-78, 80-105, 108-113, 131-143, 145-156]. In its simplest form, a plasmon can be described as a quanta of plasma oscillation or as mechanical oscillations of the electron gas of a noble metal [41-55]. Due to the strong frequency dependence of the complex permittivity of a metal, these plasmons also exhibit significant variations with frequency, giving rise to LSPR and SPR [59-72]. A common method of biosensor operation involves the exploitation of LSPR to achieve highly sensitive and selective detection capabilities [42, 62, 146-148, 156173]. This type of biosensing method can employ a variety of different nanostructure geometries, with some of the most frequently used being single LSP-supporting metal NPs [11, 49, 59-78]. LSPs remain tightly bound to the metal surface because of a high degree of surface energy confinement and have been shown to cause an associated enhanced $E$-field at the metal surface [59-100]. 
$E$-field enhancement is particularly useful in biological and chemical sensing technologies, specifically fluorescence-based detection methods, due to the relationship between fluorescence emission and $E$-field $[42,62,129]$ which will be discussed in greater detail in Chapter 2. Literature has shown that spontaneous emission of a single emitter, namely a fluorescent molecule, is significantly enhanced when coupled to photonic crystals, metallic NPs, and surface or multilayer metal structures $[66,74-75,110,146-148]$. For most noble metals, the wavelength $(\lambda)$ of LSPR typically resides in the visible to near-infrared (NIR) regime of the EM spectrum [66, 110, 146148]. Metal-coated nanopillar arrays are ideal for use in $E$-field enhancement-based sensors because they yield large, reproducible, and uniform enhancement factors over the entire surface area of the patterned lattice structure $[42,69,113]$.

Since these structures were designed specifically for biological detection, biocompatibility was of the utmost concern. For this reason, gold was chosen because it is chemically stable, nonreactive, compatible in most biological environments, easily conjugated with DNA and other biomolecules, largely resists oxidation and tarnishing effects that are characteristic of other metals like copper and silver, and has a plasmon resonance within the visible regime of the EM spectrum $[16,73,76,85,97,100,141,176-177]$. This work presents a heuristic approach for optimizing the critical lattice parameters of gold-coated nanopillar arrays of four different pillar geometries engineered to yield peak $E$-field enhancement at the surfaces of these sub-micron pillars. The research effort described herein explores the phenomena associated with the interaction of light with nanopillar arrays for optimizing optical transduction methods in modern plasmon-enhanced biosensor technologies.

\subsection{Research Goals}

The goals of this research are to use FDTD simulation software to extract the optimal lattice parameters of select gold NP lattice geometries yielding peak $E$-field enhancement, compare the resulting $E$-field enhancement factors of each optimized plasmonic lattice geometry at their respective resonance conditions, directly relate these simulated $E$-field enhancement factors to fluorescence enhancement, and select label(s) (if available/where applicable) whose emission spectra overlaps with and/or whose peak lies within the resonance condition of each optimized lattice geometry, respectively. 
The remainder of this thesis is arranged as follows: Chapter 2 details the theory behind the scope of the field of plasmonics including SPR, surface plasmon polaritons (SPP), LSPR, Mie Theory, Gans Theory, the field of PEF biosensors and their functionalization as well as the various metal architectures explored throughout plasmonics literature (i.e., thin metal films, single metal NPs, and arrays of metal NPs). In conjunction, Chapter 2 details the stimulated and spontaneous fluorescence emission enhancement (i.e., the Purcell factor) of single metal NPs and arrays of metal NPs. Finally, applicable labels such as QDs and commercially available fluorophores will be analyzed for potential integration with the optimized plasmonic lattices. Chapter 3 chronicles the FDTD method including specific software settings pertaining to the simulation region, mesh refinement, source specifications, and why it is widely used to model nanophotonic interactions. Additionally, metal skin depth and the Poynting vector is discussed and related to the Fabry- Pérot interferometer that was modeled as an introductory FDTD simulation problem with the goal of observing the cavity's resonance condition using a transverse electric (TE)-polarized dipole source. A comparison of TE and transverse magnetic (TM) modes, metal skin depth, and resonance conditions of real metals vs. a perfect electrical conductor (PEC) was performed with a presentation of the obtained cavity simulation results. Finally, the methodology detailing how the plasmonic lattice parameters were optimized is outlined. Chapter 4 presents the parametric optimization simulation results of multiple square plasmonic lattices comprised of select pillar geometries comparing the resulting $E$-field enhancement factors and intensities. The schematic view of a potential biosensor architecture incorporating an optimized plasmonic transducer can be seen in Figure 1.6. Chapter 5 outlines the conclusion of this thesis and future work including a fabrication process for the construction of the optimized plasmonic lattices with an accompanying method for characterizing NP size, morphology, and overall process flow for physical experimentation. 


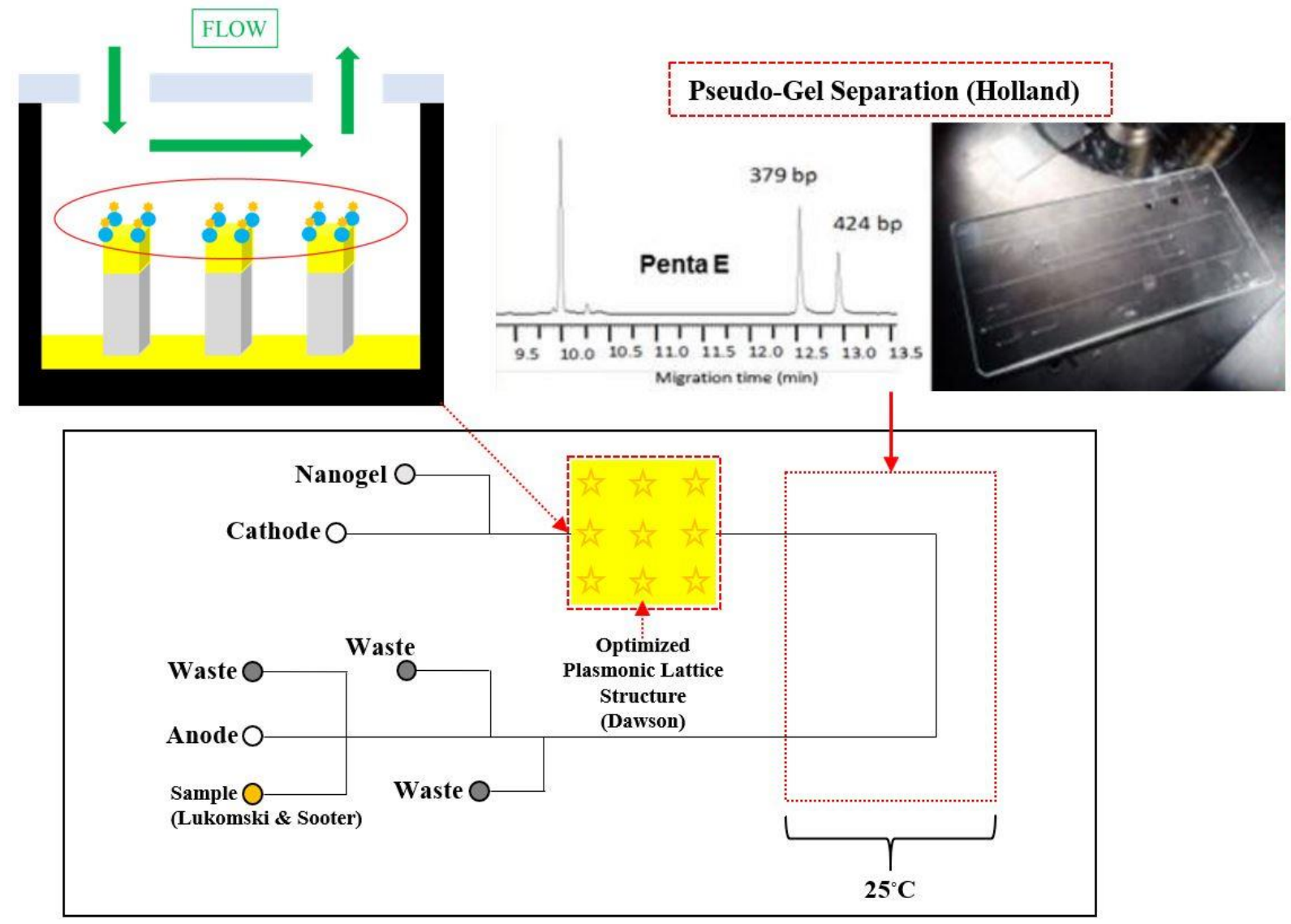

Figure 1.6 Device schematic highlighting the functional regions for portable and rapid biosensing involving microfluidic flow channels. The entire sample is pumped in, the analyte DNA undergoes polymerase chain reaction (PCR) for amplification and is then separated from other biologicals present in the sample via hydrogel, the specific bonding regions of the analyte DNA are separated from the rest of the analyte DNA strand (i.e., electrophoresis), these separated bonding regions are then conjugated with fluorescing labels engineered to bind to these regions, then passed over a plasmonic lattice structure for fluorescence enhancement (adapted from [252]). An overview of the free flow process is also included showing the labeled sample passing over the localized enhancement regions of the plasmonic lattice. 


\section{Chapter 2}

\section{Theoretical Background}

Chapter 2 delves into the theory governing the field of plasmonics including SPR, SPP, and LSPR, Mie theory, Gans theory PEF, fluorescence quenching, PEF biosensor functionalization, the Purcell factor, various metal nanostructure morphologies such as continuous metal films, single metal NPs, and arrays of metal NPs, fluorescent molecules like fluorophores and QDs, and the history of FDTD simulation software as well as why it is used in modeling the plasmonic phenomenon by its efficiency in solving Maxwell's equations. 


\subsection{Plasmonics}

\subsubsection{Surface Plasmon Resonance}

As previously mentioned, the field of plasmonics seeks to gain control over light absorption and emission by enhancing optically transduced signals (via LSPR, SPP, and/or SPR) for analysis across a vast range of applications including BFC, enhanced bioluminescence, enhanced chemiluminescence, FCS, FRET, shell-isolated nanoparticle-enhanced fluorescence (SHINEF), shell-isolated nanoparticle-enhanced Raman spectroscopy (SHINERS), SERS, surface-enhanced resonance Raman scattering (SERRS), tip-enhanced Raman spectroscopy (TERS), etc. [48, 59-73, 70, 76-87, 90-108, 126]. The high sensitivity of LSPR and SPR to refractive index changes via biomolecular interactions provide a powerful means for improving label-free and labeled biodetection modalities previously discussed in Section 1.4.1 and 1.4.2. Since its commercial launch in 1990, SPR sensing has proven to be key apparatus in the research of clinical analyses, environmental monitoring, food quality, and pharmaceutical development $[11,59-73,80$, 178180]. SPR occurs when the frequency of an evanescent EM wave propagating incident on a metaldielectric interface is in resonance with the collective oscillation frequency of a metal's conduction band electrons [70, 80, 93-95, 103]. SPR sensor construction generally utilizes the Kretschmann configuration whereby a high refractive index prism coupler $\left(\eta_{p}\right)$ is used to guide the excited TM nature of the SP wave $(k)$ to a smooth thin metal film [70, 80, 93, 103].

The Kretschmann configuration exploits the higher refractive index of the prism compared to the refractive index of the surrounding medium $\left(\eta_{P}>\eta_{D}\right)$ at a specific incident angle $(\theta)$ and wavelength of incident light $(\lambda)$ [59-73]. By employing a high refractive index material, the Kretschmann configuration permits the wave vector of incident light $(k)$ to couple with the wave vector of the surface plasmon $\left(\beta_{S P}\right)$ at the SPR resonance condition. Resonance at the metaldielectric interface can be optimally coupled by parallel $(p)$-polarized light at a given wavelength and angle of incidence and occurs when the SP wave vector equals the propagation constant of the incident light [59-73, 80, 93-95]. 
The plasmon resonance condition of the Kretschmann configuration is postulated below:

$k_{X}=\beta_{S P}$ (propagation constant)

$\beta_{S P}=\operatorname{Re}\left\{\frac{2 \pi}{\lambda} \sqrt{\frac{\varepsilon_{M} \varepsilon_{D}}{\varepsilon_{M}+\varepsilon_{D}}}\right\}$

Here, $\varepsilon_{M}$ is the real part value of the dielectric constant of the metal and $\varepsilon_{D}$ is the real part value of the dielectric constant of the surrounding medium $[80,103]$.

$\frac{2 \pi}{\lambda} \cdot \eta_{p} \cdot \sin (\theta)=\operatorname{Re}\left\{\frac{2 \pi}{\lambda} \sqrt{\frac{\varepsilon_{M} \varepsilon_{D}}{\varepsilon_{M}+\varepsilon_{D}}}\right\}$

Rearranging (3) solving for the SPR incident angle:

$\theta=\sin ^{-1}\left[\left(\frac{1}{\eta_{p}}\right) \cdot \operatorname{Re} \sqrt{\frac{\varepsilon_{M} \varepsilon_{D}}{\varepsilon_{M}+\varepsilon_{D}}}\right]$

Another factor of importance is the propagation length $\left(L_{X}\right)$ of the SP wave:

$L_{X}=\frac{\pi}{2 \lambda} \frac{\varepsilon_{i M}}{\varepsilon_{M}^{2}}\left(\frac{\varepsilon_{M} \varepsilon_{D}}{\varepsilon_{M}+\varepsilon_{D}}\right)^{3 / 2}$

In (5) above, $\varepsilon_{i M}$ is the imaginary value of the metal-dielectric constant which corresponds with the broadening of the resonance peak (i.e., damping) [80, 103]. In the generation of SP modes, the wavelength of interest requires the configuration be tailored to match the propagation constant of the incident light at a specific angle of incidence [80]. Exploration priority and sensor configuration is determined by the light source preference (i.e., for a polychromatic light source, determining the SPR reflectivity profile requires wavelength interrogation while, for a monochromatic light source, the SPR reflectivity profile requires angular interrogation) [41, 65, 80, 103]. Figure 2.1 delineates the Kretschmann configuration. 


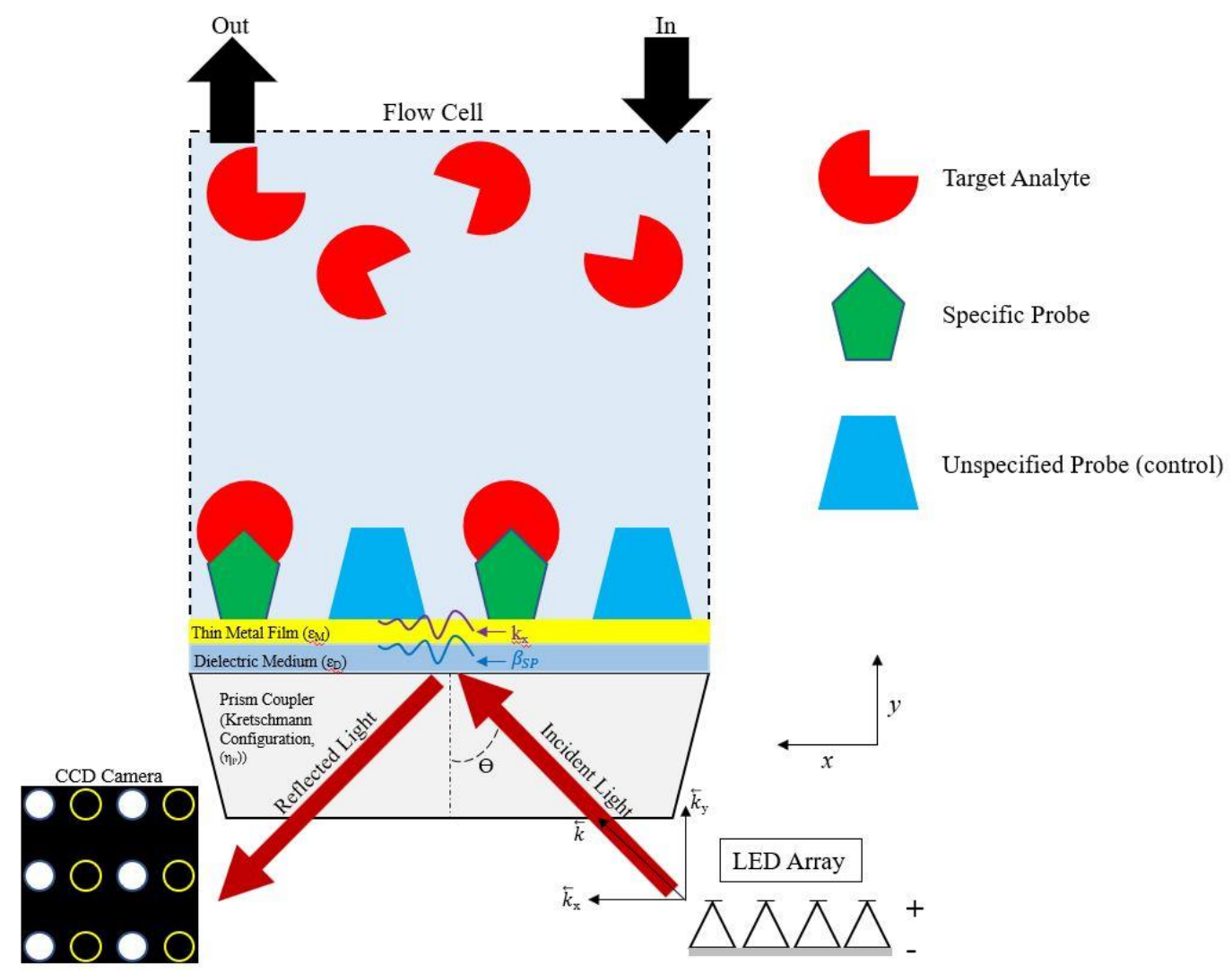

Figure 2.1 Kretschmann configuration for analyte analysis using SPR (adapted from [41], [80], and [103]).

The high refractive index prism is connected to a flow cell where coupling of incident EM radiation to the surface plasmons of a thin metal film by evanescent waves occurs. $p$-polarized light is guided to the prism coupler attached to a flow cell containing the biomolecular probe(s). These reflections are captured by the CCD camera recorded as variations in reflection intensities at a fixed incidence for specified areas of interest. Specific interactions with the analyte of interest causes a reaction from the specific probe resulting in localized changes in the intensity of the reflected light $[41,80]$. These changes in reflection intensities translate into a black-white image contrast that the CCD camera then uses to render a digital image of in real-time [80]. 


\subsubsection{Surface Plasmon Polaritons}

SPPs are TM longitudinal electron density fluctuations (plasma oscillations) propagating along a metal or dielectric surface at an eigenfrequency $\left(\omega_{p}\right)$ caused via optical excitation $[93,95$, 101, 103, 146]. SPPs, specifically, consist of a surface plasmon coupled with a photon (polariton) and can be seen in Figure $2.2[101,103,146]$.
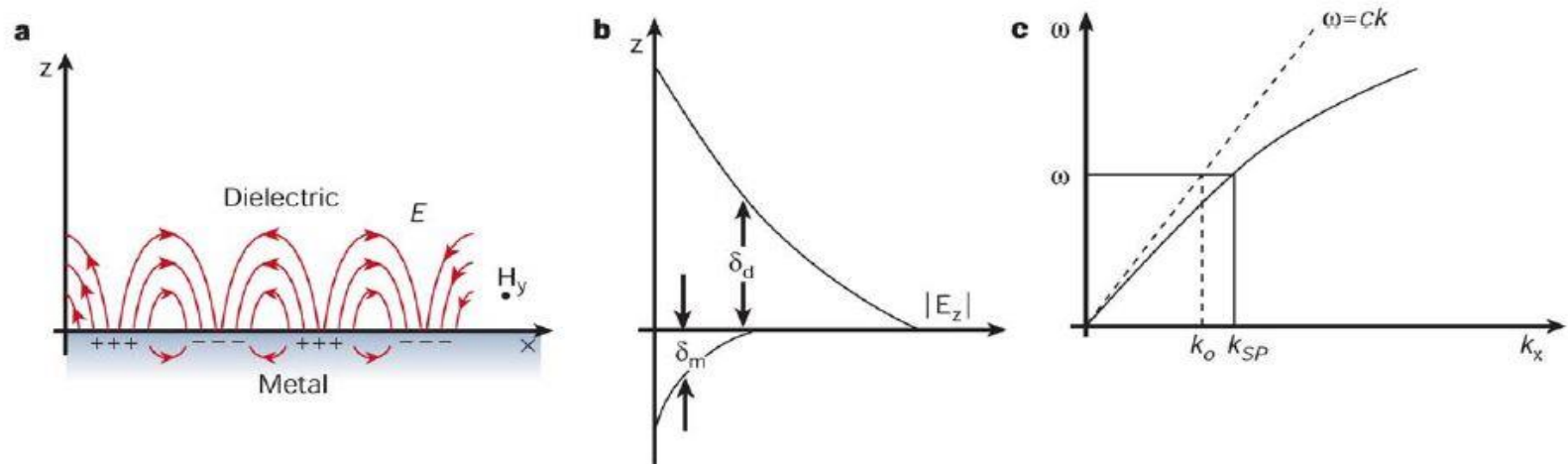

Figure 2.2 SPP wave propagation and field decay length over a given distance [101].

Maxwell's theory of electromagnetism shows that these EM surface waves can propagate along a metal surface across a broad range of eigenfrequencies $\left(0 \leq \omega \geq \omega_{p} \sqrt{2}\right)$ [80, 101, 103]. By combining Maxwell's two curl equations with $J_{\text {ext }}=\rho_{\text {ext }}=0$ :

$\nabla \times \nabla \times \mathrm{E}=-\mu_{0} \frac{\partial^{2}}{\partial t^{2}} \rightarrow \nabla^{2} E-\frac{\varepsilon}{c^{2}} \frac{\partial^{2} D}{\partial t^{2}}=0$

Assuming harmonic time dependence where $k_{0}=\omega / c$ results in the Helmholtz equation :

$E(r, t)=E(r) e^{-i w t} \rightarrow \nabla^{2} E-k_{0}^{2} \varepsilon E=0$

For the defined geometry, $\varepsilon=\varepsilon(\mathrm{z})$ wave propagation can be described as the following:

$E(x, y, z)=E(z) e^{i \beta x}$

The exponential above contains the propagation constant presented in (1). 
Inserting (8) into the Helmholtz equation results in the wave equation:

$\frac{\partial^{2} E(z)}{\partial z^{2}}+\left(k_{0}^{2}-\beta^{2}\right) E=0$

The wave equation above contains two sets of solutions:

$$
\begin{array}{lrl}
E_{x}, E_{Z}, H_{y} \neq 0 & \text { (TM }(p) \text { Mode) } \\
H_{x}, H_{z}, E_{y} \neq 0 & \text { (TE }(s) \text { Mode) }
\end{array}
$$

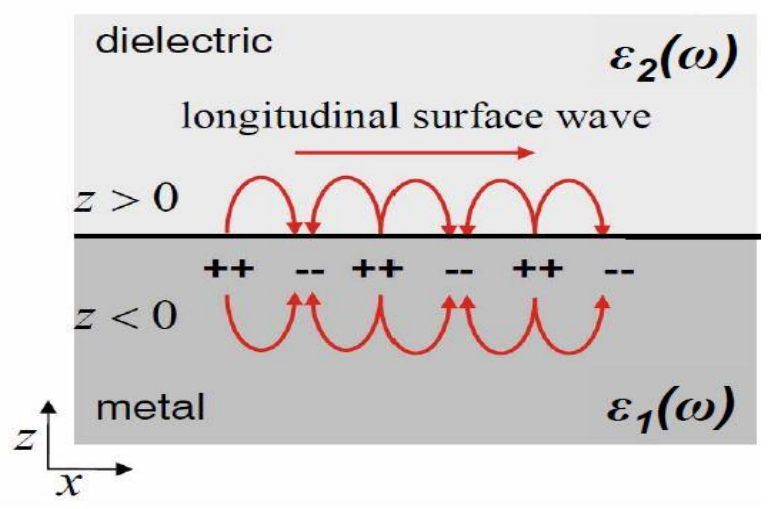

Figure 2.3 Confined longitudinal wave nature of the TM SPP modes [101].

Note that SPPs only exist for the TM polarization mode [101, 103]. For the TM SPP modes at a metal/dielectric interface (Figure 2.3), the longitudinal SPP waves are governed by the following equations:

$$
\begin{aligned}
& H_{y}(z)=A_{2} e^{i \beta x} e^{-i k_{2, z} z} \\
& E_{x}(z)=i A_{2} \frac{1}{\omega \varepsilon_{0} \varepsilon_{2}} k_{2} e^{i \beta x} e^{-k_{2, z} z} \\
& E_{z}(z)=-A_{1} \frac{1}{\omega \varepsilon_{0} \varepsilon_{2}} e^{i \beta x} e^{-k_{2, z} z} \\
& H_{y}(z)=A_{1} e^{i \beta x} e^{k_{1, z} z} \\
& E_{x}(z)=-i A_{1} \frac{1}{\omega \varepsilon_{0} \varepsilon_{1}} k_{1} e^{i \beta x} e^{k_{1, z} z} \\
& E_{z}(z)=-A_{2} \frac{1}{\omega \varepsilon_{0} \varepsilon_{1}} e^{i \beta x} e^{k_{1, z} z}
\end{aligned}
$$


Above, $\operatorname{Re}\left[\varepsilon_{1}\right]<0$ and $1 / k_{z}$ define the evanescent decay length perpendicular to the metal/dielectric interface leading to wave confinement. The dispersion relations (valid for both real and complex values of $\varepsilon$ ) contained in equations (12) - (17) are as follows:

$\beta=k_{0} \sqrt{\frac{\varepsilon_{1} \varepsilon_{2}}{\varepsilon_{1}+\varepsilon_{2}}}$

$k_{i, z}=k_{0} \sqrt{\frac{\varepsilon_{i}^{2}}{\varepsilon_{1}+\varepsilon_{2}}}$

Figure 2.4 details the confined SPP wave vector and charge oscillation across a metal/dielectric interface.
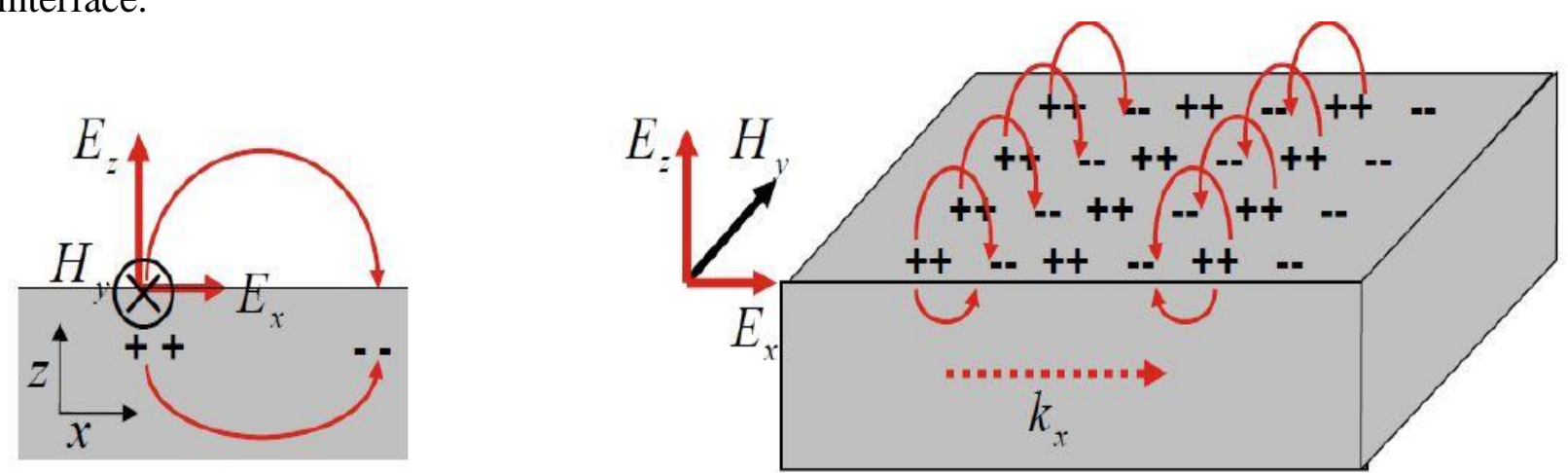

Figure 2.4 Confined SPP wave vector and charge oscillation across a metal/dielectric interface in accordance with Helmholtz [101].

Figure 2.5 delineates the locations of plasmon resonance conditions for various metal surfaces in a vacuum. 


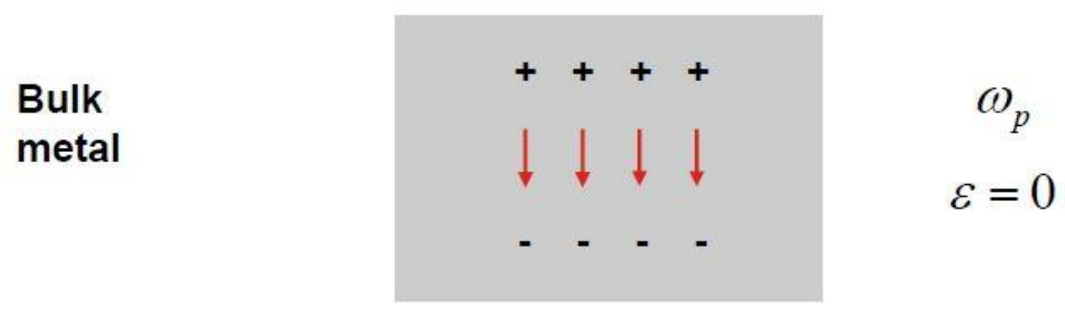

Metal

surface

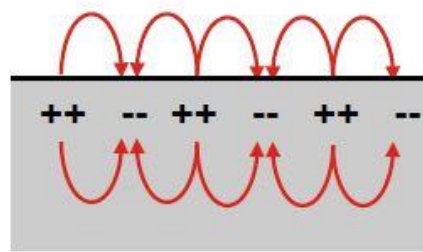

$$
\varepsilon=-1 \underset{\substack{\text { drude } \\ \text { model }}}{\longrightarrow} \omega_{p} / \sqrt{2}
$$

\section{Metal sphere localized SPPs}

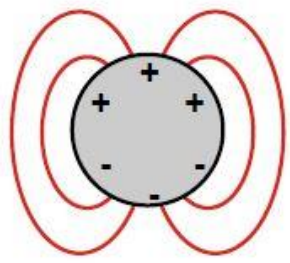

$$
\varepsilon=-2 \underset{\begin{array}{c}
\text { drude } \\
\text { model }
\end{array}}{\longrightarrow} \omega_{p} / \sqrt{3}
$$

\section{Figure 2.5 Regions of SPP resonance via irradiation for bulk materials, thin metal films, and spherical metal NPs [101].}

Along with plasmon polariton generation, other elementary excitations including lattice vibrations (phonons) and bound states between excited electron-hole pairs (excitons) also arise from incident EM radiation [95, 101, 103]. The amplitude of the propagating SPP wave decays exponentially increasing with distance and can be analogized to the ripple effect observed in a body of water. As shown, the SPPs are tightly confined to the metal surface or metal-dielectric interface leading to an enhancement of the EM fields at the surface or interface $[95,101,103]$. The application of devices employing the high sensitivity of SPR and/or SPPs are widely used across bio- and chemi-sensor modalities including fluorescence spectroscopy, LSPR, SERS, surface adsorbates, surface roughness, TERS, waveguides, etc. and provide significant flexibility in the engineering of optical transducers $[11,59-73,80,101,103,146]$. In the consideration of SPPs, one must first consider the dielectric constant of the metal:

$$
\varepsilon_{m}(\omega)=1-\left(\frac{\omega_{P}}{\omega}\right)^{2}
$$


In the case where $\omega<\omega_{p} \rightarrow \varepsilon_{m}<0$, there are no propagating EM modes since the plane wave vector of light in the medium is imaginary. In the case where $\omega>\omega_{p} \rightarrow \varepsilon_{m} \rightarrow 1$, the SPPs are altered by intraband transitions within the noble metal [101]. Since the oscillating $E$-field of the irradiating plane wave is responsible for the excited SPs, light possessing a high angle of incidence (i.e., where the wave vector $(k)$ is nearly parallel to the surface), coupling between incident light and the SP modes is most efficient $[80,93,95,101,103]$. The wavelength of the propagating SPP along a metal or dielectric surface is the following:

$\lambda_{s p p}=\lambda_{0} \sqrt{\frac{\varepsilon_{M}+\varepsilon_{D}}{\varepsilon_{M} \varepsilon_{D}}}$

Contained in (21) is the wavelength of the incident plane wave $\left(\lambda_{0}\right)$, the dielectric constant of the medium $\left(\varepsilon_{M}\right)$, and the dielectric constant of the metal $\left(\varepsilon_{D}\right)$. When the SP mode is confined to a NP whose size is comparable to the wavelength of incidence (i.e., the free electrons of the NP participate in resonant collective oscillation) localized regions of surface plasmon resonance are produced [61, 68, 87, 93, 95, 101, 115, 131, 137, 172-173].

\subsubsection{Localized Surface Plasmon Resonance}

LSPR occurs when the frequency of an incident EM wave resonates with the collective conduction band electrons of a metallic NP [4-10, 41-55, 61, 68]. The oscillation of the electron cloud causes a charge imbalance and produces localized regions of enhanced $E$-field capable of coupling with an optical wave vector $[9,75,115,132,147,152,172-173]$. The manipulability and predictability of LSPR renders itself useful for applications like SERS and fluorescence spectroscopy because of the strong localized regions of enhanced $E$-field. The overlap between a metal's LSPR condition and the molecular absorption and emission spectra of a fluorescent label has been experimentally shown to yield the highest fluorescence enhancement $[42,61,82,111$, 134, 138, 150-151, 175]. Additionally, metal nanostructures are known for their ability to interact with organic compounds to significantly improve the photostability and fluorescence intensity of many fluorescent dye molecules [12, 127, 129, 135, 174]. 
The coupling of light with LSPs can strongly confine of EM fields capable of interacting with fluorophores at their absorption and emission wavelengths [41-42, 61, 93, 95, 175]. This interaction alters transitions between the ground state and excited sates of a fluorophore with excitation originating with an irradiated incident EM wave possessing an $E$-field at the absorption wavelength $\left(\lambda_{a b}\right)$ of the fluorophore $[12,42,61,68,82,111,134,138,150-151,175]$. Figure 2.6 provides visualization of an irradiated gold NP and the resulting LSPR excitation among other transpiring physical processes. Sensing applications of LSPR-active NPs involve the detection of changes in the bulk refractive index of the experimental environment via shifts in LSPR peak wavelength $[111,134,138,150-151,175]$. Shifts in the LSPR peak are detected through the use of spectral extinction measurements on a dense metal film or a single metal NP. NP features can either be immobilized on the substrate surface or directly patterned via electron beam lithography, micro-contact $(\mu \mathrm{CP})$ printing, photolithography, etc. and then coated with a plasmon-capable metal [34, 82, 154, 187]. Physical LSPR sensor construction of the plasmonic lattices studied within the scope of this thesis will be detailed in Chapter 3.

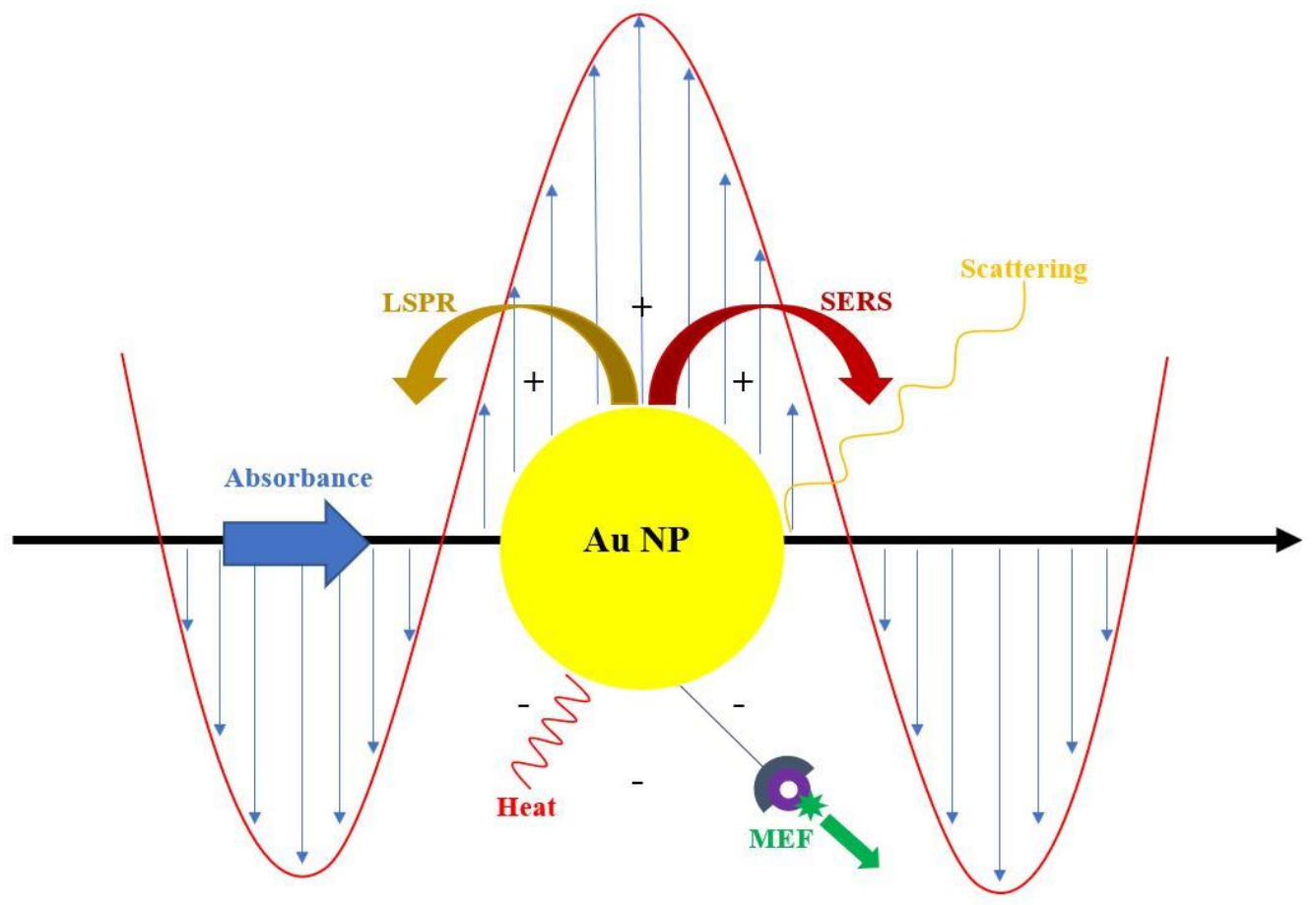

Figure 2.6 Metal NP electron cloud oscillation resulting from incident EM radiation. Accompanying radiative and non-radiative processes arising from an irradiated metal NP including heat dissipation, MEF, LSPR, SERS, and electron scattering are also shown (adapted from [175]). 


\subsubsection{Mie Theory}

In the early portion of the $20^{\text {th }}$ century, Gustav Mie postulated an analytical approach to solving Maxwell's equations governing light absorption and scattering by a spherical NP where the size of the NP is much smaller than the wavelength of light $(d<<\lambda)[61,106]$. Mie found that the scattering fields produced by a plane wave incident on a homogenous conducting sphere behave similar to Rayleigh scattering, and that the total absorption, extinction, and scattering crosssections can be described as:

$$
\begin{aligned}
& \sigma_{a b s}=\sigma_{\text {ext }}-\sigma_{\text {scat }} \\
& \sigma_{s c a t}=\frac{2 \pi}{|k|^{2}} \sum_{L=1}^{\infty}(2 L+1)\left(\left|a_{L}\right|^{2}+\left|b_{L}\right|^{2}\right) \\
& \sigma_{e x t}=\frac{2 \pi}{|k|^{2}} \sum_{L=1}^{\infty}(2 L+1)\left[\operatorname{Re}\left(a_{L}+b_{L}\right)\right]
\end{aligned}
$$

In these expressions, $k$ is the incident wave vector and $L$ are integers values representing scattering dipole, quadrupole, and other higher multipoles [61, 106]. Additionally, the parameters $a_{L}$ and $b_{L}$ are composed of the Riccati-Bessel functions $\psi_{L}$ and $\chi_{L}[61,188]$ :

$$
\begin{aligned}
& a_{L}=\frac{m \psi_{L}(m x) \psi_{L}^{\prime}(x)-\psi_{L}^{\prime}(m x) \psi_{L}(x)}{m \psi_{L}(m x) \chi_{L}^{\prime}(x)-\psi_{L}^{\prime}(m x) \chi_{L}(x)} \\
& b_{L}=\frac{\psi_{L}(m x) \psi_{L}^{\prime}(x)-m \psi_{L}^{\prime}(m x) \psi_{L}(x)}{\psi_{L}(m x) \chi_{L}^{\prime}(x)-m \psi_{L}^{\prime}(m x) \chi_{L}(x)}
\end{aligned}
$$

Above, $m=\tilde{n} / n_{\mathrm{m}}$ where $\tilde{n}=n_{R}+i n_{I}$ is the complex refractive index of the metal, $n_{\mathrm{m}}$ is the real refractive index of the surrounding medium, $x=k_{m} r$ where $r$ is the radius of the sphere, and $k_{m}=$ $2 \pi / \lambda_{m}$ represents the wavenumber of the medium $[61,188]$. In cases where the NP is very small in comparison to wavelength $(x<<1)$, the Riccati-Bessel functions can then be approximated by power series [61, 188]. 
Keeping the terms on the order $x^{3}$, equations (25) and (26) can be simplified to (27) and (28):

$a_{1} \approx-\frac{i 2 x^{3}}{3} \frac{n_{R}^{2}-n_{I}^{2}+i 2 n_{R} n_{I}-n_{m}^{2}}{n_{R}^{2}-n_{I}^{2}+i 2 n_{R} n_{I}+2 n_{m}^{2}}$

$b_{1} \approx 0$

Accounting for the complex dielectric function of the metal:

$\tilde{\varepsilon}=\varepsilon_{1}+i \varepsilon_{2}$

$\varepsilon_{1}=n_{R}^{2}-n_{1}^{2}$

$\varepsilon_{2}=2 n_{R} n_{1}$

Switching back to the dielectric function of the medium from refractive index:

$\varepsilon_{m}=n_{m}^{2}$

Substitution of the above conversion into (25):

$a_{1} \approx \frac{2 x^{3}}{3} \frac{-i \varepsilon_{1}^{2}-i \varepsilon_{1} \varepsilon_{m}+3 \varepsilon_{2} \varepsilon_{m}-i \varepsilon_{2}^{2}+i 2 \varepsilon_{m}^{2}}{\left(\varepsilon_{1}+2 \varepsilon_{m}\right)^{2}+\left(\varepsilon_{2}\right)^{2}}$

Substituting (33) into (24) taking the dipole term exclusively yields the NP plasmon resonance. The extinction cross-section then becomes:

$\sigma_{e x t}=\frac{18 \pi \varepsilon_{m}^{3 / 2} V}{\lambda} \frac{\varepsilon_{2}(\lambda)}{\left[\varepsilon_{1}(\lambda)+2 \varepsilon_{m}\right]^{2}+\varepsilon_{2}(\lambda)^{2}}$

where $V$ is the volume of the NP. 
Through similar substitution, the following scattering cross-section becomes:

$$
\sigma_{s c a t}=\frac{32 \pi^{4} \varepsilon_{m}^{2} V^{2}}{\lambda^{4}} \frac{\left(\varepsilon_{1}-\varepsilon_{m}\right)^{2}+\left(\varepsilon_{2}\right)^{2}}{\left(\varepsilon_{1}+2 \varepsilon_{m}\right)^{2}+\left(\varepsilon_{2}\right)^{2}}
$$

Despite the fact that these approximations strictly apply to very small NP ( $<10 \mathrm{~nm}$ in diameter), the predictions offered on dielectric sensitivity are still accurate in the application to larger NPs [61]. When considering bulk (pertaining to materials with a large extent in all three dimensions compared to the wavelength light) plasmons, these electron oscillations occur at the plasma frequency of the metal with the following energy:

$E_{P}=\hbar \sqrt{\frac{n e^{2}}{m \varepsilon_{0}}}$

In (36), $\varepsilon_{0}$ is free-space permittivity $\left(8.85 \times 10^{-12} \mathrm{~F} / \mathrm{m}\right), n$ is the electron density, $e$ is the electric charge, and $m$ is the mass of an electron $\left(9.10938356 \times 10^{-31} \mathrm{~kg}\right)$ [61]. The plasma frequency of a metal is shown below:

$\omega_{P}=\sqrt{\frac{n e^{2}}{m \varepsilon_{0}}}$

It can be seen that $\omega_{P}$ corresponds to a species' typical electrostatic oscillation in response to a small separation of charge [119]. The observance of plasma oscillations only occurs when studied over periods of time $(\tau)$ longer than the lifetime of the plasmon $\left(1 / \tau_{P}\right)$ and if an external action changes the system at a rate less than or equal to $\omega_{P}$ [119]. The Drude model provides an accurate approximation of noble metal conductivity and dispersion describing the electronic structure of metals accounting for collisions between free-moving electrons and a lattice of heavy, stationary ionic cores. The Drude model:

$$
\varepsilon_{m}=1-\frac{\omega_{P}^{2}}{\omega^{2}+\gamma^{2}}=1-\frac{\omega_{P}^{2}}{\omega^{2}+j \omega \gamma}
$$


Here again, $\omega_{P}$ is the plasma frequency of the metal and $\gamma$ is the damping parameter of the bulk metal [119]. For visible and NIR wavelengths, where $\gamma<<\omega_{P}$, the Drude model can be further simplified:

$\varepsilon_{1}=1-\frac{\omega_{P}^{2}}{\omega^{2}}$

Setting $\varepsilon_{1}$ equal to the resonant condition $\left(-2 \varepsilon_{M}\right)$ where the extinction cross-section of the metal NP is maximized according to Mie theory [61, 119], the following expression for the LSPR peak frequency $\left(\omega_{\max }\right)$ is obtained:

$\omega_{\max }=\frac{\omega_{P}}{\sqrt{2 \varepsilon_{m}+1}}$

Converting angular frequency to wavelength $(\lambda=2 \pi c / \omega)$ and dielectric constant to refractive index $\left(\varepsilon_{M}=n^{2}\right)$ yields:

$\lambda_{\max }=\lambda_{P} \sqrt{2 n_{m}^{2}+1}$

Again, $\lambda_{\max }$ is the LSPR peak wavelength with $\lambda_{\mathrm{P}}$ is the equivalent wavelength of the bulk metal's plasma frequency. Referencing experimental values determined by [176] and [177] it can be seen that the dependence of $\lambda_{\max }$ on $n_{m}$ should be linear in approximation at optical frequencies [61].

\subsubsection{Gans Theory}

Mie's formulations are only applicable to spherical metal NPs $[61,107]$. In an attempt to

apply Mie's theoretical model to small spheroidal particle geometries, Richard Gans in 1912 proposed a new theory applicable to prolate spheroids of any aspect ratio (AR). 
Analogous to the absorption cross-section put forth by Mie, Gans found that, for a prolate (i.e., generated by the revolution of an ellipse about its longer axis) spheroid is:

$\sigma_{a b s}=\frac{\omega}{3 c} \varepsilon_{m}^{3 / 2} V \sum_{j} \frac{\left(1 / P_{j}^{2}\right) \varepsilon_{2}}{\left\{\varepsilon_{1}+\left[\frac{1-P_{j}}{P_{j}}\right] \varepsilon_{m}\right\}^{2}+\varepsilon_{2}^{2}}$

Three dimensions of the NP are considered here and account for the sum over $j . P_{j}$ includes $P_{A}$, $P_{B}$, and $P_{C}$ (depolarization factors) for each of the particle's three axes where $\mathrm{A}>\mathrm{B}=\mathrm{C}$ in the case of the aforementioned prolate spheroid $[61,107]$. Anisotropic alteration of $\varepsilon_{1}$ and $\varepsilon_{2}$ via these depolarization factors occurs resulting in LSPR peaks:

$P_{A}=\frac{1-e^{2}}{e^{2}}\left[\frac{1}{2 e} \ln \left(\frac{1+e}{1-e}\right)-1\right]$

$P_{B}=P_{C}=\frac{1-P_{A}}{2}$

$e$ is a factor that includes NP AR and is expressed as:

$e=\left[1-\left(\frac{B}{A}\right)^{2}\right]^{1 / 2}=\left(1-\frac{1}{R^{2}}\right)^{1 / 2}$

The resulting spectrum from (42) contains two peaks - one corresponding to the $x$ and $y$ contributions of the transverse plasmon modes to the total sum and the other corresponding to the longitudinal plasmon sum from the z-direction [61]. Equation (42) also provides insight into the role AR plays on the LSPR peak wavelength. The factor $\varepsilon_{m}$ is equal to 2 for spherical particles. However, this quantity equals $\left[\left(1-P_{j}\right) / P_{j}\right]$ for prolate spheroids and increases with AR (i.e., this weighting factor can be significantly larger than 2) [61]. As a result, red shifting of the LSPR peak occurs with an increase in particle AR coupled with an increased sensitivity to the surrounding medium, specifically the dielectric constant of that medium [61, 77-78]. 
Particle morphology also plays a substantial role in the determination of the LSPR condition for metal NPs but, contrary to those of spheres (Mie) and prolate spheroids (Gans), the LSPR spectrum of other NP geometries must be explored numerically [61]. Numerical methods available for approaching non-spheroid geometries include the discrete dipole approximation (DDA), finite element method (FEM), and FDTD with the latter discussed in further detail in Chapter 3. The coupling between the frequency of fluorescence emission and NP resonance can cause the metal to radiate light with enhanced intensity at the same frequency (i.e., elastic scattering) as the frequency of fluorescence emission [4-10, 12-13, 42, 62, 72, 82, 96, 110-111, 131-134, 138-140]. This phenomenon is often referred to as metal-enhanced fluorescence (MEF), a sub-section of PEF [8-9, 42, 66, 91, 143, 149, 164, 171].

\subsubsection{Plasmon-Enhanced Fluorescence}

The first PEF biosensor was implemented in the early 1990s reintroduced less than a decade later as surface plasmon-enhanced fluorescence spectroscopy (SPFS) [42]. PEF pertains to the fabrication of specific nanostructures tailored to enhance the fluorescence emission process aimed at understanding the interactions or coupling between plasmonic nanomaterials and individual (or an ensemble of) emitters $[42,62,72,82,96]$. The PEF approach takes advantage of fluorescence enhancement by probing a metal sensor surface with SPPs that are excited in resonance with the absorption wavelength of the fluorophore used and then utilizing these SPPs to collect fluorescent light at the emission wavelength of the fluorophore (i.e., surface plasmoncoupled emission (SPCE) $[42,161]$. This aforementioned methodology is rooted in ab initio electronic structures, quantum electrodynamics, and quantum plasmonics [42]. PEF, originally referred to as surface-enhanced fluorescence (SEF), is a spectroscopy technique that has had a profound impact in the stimulation of new analytical detection, single molecule detection and imaging, diagnostics, and nanoplasmonic fabrication protocols [8-9, 42, 66, 91]. The obtainable single-molecule sensitivity allows for the detection of weakly emitting low QE labels otherwise undetectable by more conventional fluorescence detection methods [12-13, 110-111, 131-134, 138-140]. PEF possesses a wide scope of applications including biomedical diagnostics, enhanced solar cell and organic photovoltaic performance, and an expansion of bio- and chemosensors [8, 41-42, 61, 105]. 
Recent reviews of plasmonic biosensor applications have been presented by [37], [41-42], and [91]. One of the strategies to improve the sensitivity of fluorescence-based biosensors is by placing the fluorophore in the high $E$-field generated by light confinement within metallic NPs, therefore improving the detection sensitivity by enhancing fluorescence emission. The coupling between the emitted frequencies of a fluorophore and the plasma resonance of the metal nanostructure can cause light to be radiated by the metal with an enhanced intensity at the same frequency of the fluorescence emission $[8,41-42,61,105]$. This experimental and theoretical study of PEF is commonly known as metal-enhanced fluorescence (MEF) [42, 62].

MEF is a special case of plasmonic biosensors involving the study of fluorophores near metal nanostructures. The increased $E$-field around the nano-patterned plasmonic lattice directly improves the excitation and emission enhancement of the fluorophores, which in turn reduces the LOD $[6,8,41-42,62,110,158,162]$. Fluorescence enhancement is due to the localized surface plasmon polaritons associated with metal nanostructures, which increases the $E$-field at and around the nanostructure surface. The fluorescent molecules are modified by the increased $E$-field around the nanostructures, resulting in an enhanced excitation rate and subsequent fluorescence emission enhancment. As a result, MEF is widely investigated for labeled biosensor applications, particularly for fluorescent dyes with low quantum yield $[6,42,62,110,158,162]$. The PEF experimental process attempts to correlate the role external excitation frequency, source polarization, optical properties of the emitter (e.g., extinction coefficient, lifetime, and quantum yield), spatial orientation, and coupling to LSPR 'hotspots' play on the fluorescence emission process $[42,51,61-62]$. Surface plasmon-induced augmentation of a fluorophore's excitation and decay rates can be classically illustrated by Maxwell's equations by using the absorption $\left(\mu_{a b}\right)$ and emission $\left(\mu_{e m}\right)$ electric dipole moments of the fluorophore [42]. The excitation rate of a fluorophore $\left(\gamma_{e}\right)$ irradiated by an incident EM wave possessing an $E$-field $(E)$ at the fluorophore's $\lambda_{a b}$ can be expressed as the following:

$\gamma_{e} \propto\left|E \times \mu_{a b}\right|^{2}$

Fluorescence yield is a key parameter of an elementary emitter and is the product of two factors: 1) $E$-field intensity enhancement factor $(G)$ for the excitation light source, and 2) the quantum yield $(Q)$ of the emitter near metallic NPs $[42,62]$. 
This relationship is presented below:

$F=G Q=\left|\frac{E}{E_{0}}\right|^{2} \times\left(\frac{\gamma_{r}}{\gamma_{r}+\gamma_{n r}}=\frac{\gamma_{r}}{\gamma_{\text {total }}}\right)$

Here, $F$ is the fluorescence enhancement factor, $\gamma_{r}$ is the radiative decay rate of the emitter, $\gamma_{n r}$ is the non-radiative decay rate of the emitter, $\gamma_{\text {total }}$ is the total decay rate (the sum of the radiative and non-radiative decay rates) of the emitter, $E$ is the amplitude of the enhanced $E$-field in the presence of metal $\mathrm{NP}(\mathrm{s})$, and $E_{0}$ is the default source amplitude of the incident radiation (typically $1 \mathrm{~V} / \mathrm{m}$ ) [62]. The increased $E$-field creates an additional radiative decay source when the fluorescent molecule is near metallic nanostructures. Expanding upon (47) and accounting for this produces a modified quantum yield $(\eta)[42,129-133]$ :

$\eta=\frac{\frac{\gamma_{r}}{\gamma_{r}^{0}}}{\left(\frac{\gamma_{r}}{\gamma_{r}^{0}}\right)+\left(\frac{\gamma_{a b s}}{\gamma_{r}^{0}}\right)+\left(\frac{\left(1-\eta_{0}\right)}{\eta_{0}}\right)}$

$\gamma_{r}+\gamma_{n r}=\gamma_{a b s}+\gamma_{n r}^{0}$

$\gamma_{a b s}$ is the metal-enhanced absorption decay rate that directly competes with the NP-enhanced radiative decay rate, shortens the excited lifetime of the fluorophore, and reduces the amount of optical energy stored vs. the amount of optical energy lost to the surrounding environment (i.e., the quality factor $(Q)$ ). Taking into consideration the directionality of surface-plasmon coupled emission $(f)$, the metal NP-modified radiative $\left(\gamma_{r}\right)$ and non-radiative decay rates $\left(\gamma_{n r}\right)$ resulting from an increased local density of optical states (LDOS) at the emission wavelength of the fluorophore results in:

$$
\begin{aligned}
& F_{E}=\left|\frac{E}{E_{0}}\right|^{2} \times\left\{\frac{\frac{\gamma_{r}}{\gamma_{r}^{0}}}{\left(\frac{\gamma_{r}}{\gamma_{r}^{0}}\right)+\left(\frac{\gamma_{a b s}}{\gamma_{r}^{0}}\right)+\left(\frac{\left(1-\eta_{0}\right)}{\eta_{0}}\right)}\right\} \times f \\
& f=\max \left\{\frac{4 \pi \gamma_{r}(\theta, \phi)}{\iint \gamma_{r}(\theta, \phi) \sin (\theta) d \theta d \phi}\right\}
\end{aligned}
$$


Denoted in (50) are the intrinsic radiative and non-radiative decay rates of the fluorophore superscripted with ' 0 ' as well as the intrinsic quantum yield of the fluorophore $\left(\eta_{0}\right)$. Note that $\gamma_{r}$ and $\gamma_{n r}$ are associated with the plasmon-enhanced $E$-field intensity [42, 129-133]. Some examples of $\gamma_{n r}$ include excimer formation, exciplex formation, electron transfer, FRET, intramolecular charge transfer, internal conversion, photochemical transformation, and proton transfer [127, 129, $174,183,185]$. Here, $\gamma_{r}(\theta, \phi)$ is the radiative decay rate density at the emission wavelength integrated over all polar $(\theta)$ and azimuthal $(\phi)$ angles, and $\gamma_{\mathrm{e}}$ is the plasmon-enhanced excitation rate [129-133]. Encompassing (42), (43), and (45) yields the enhancement factor of detected fluorescence intensity with respect to the measured fluorescence intensity of a free fluorophore in a homogeneous aqueous environment:

$E F \propto \frac{\gamma_{e}}{\gamma_{e}^{0}} \times \frac{\eta}{\eta_{0}} \times f$

Although the above equations accurately describe fluorescence intensity, there are various other factors on which fluorescence enhancement depends such as the intrinsic quantum yield of the emitter, its concentration in solution, the surrounding medium, etc. [42, 174, 183, 185]. The lifetime a fluorophore's excited state is:

$\tau=\frac{1}{\gamma_{r}+\gamma_{n r}}$

Figure 2.7 depicts an excited fluorophore with a visualization of the electronic and vibrational energy levels of the QDs used in experimentation within the scope of this thesis. 


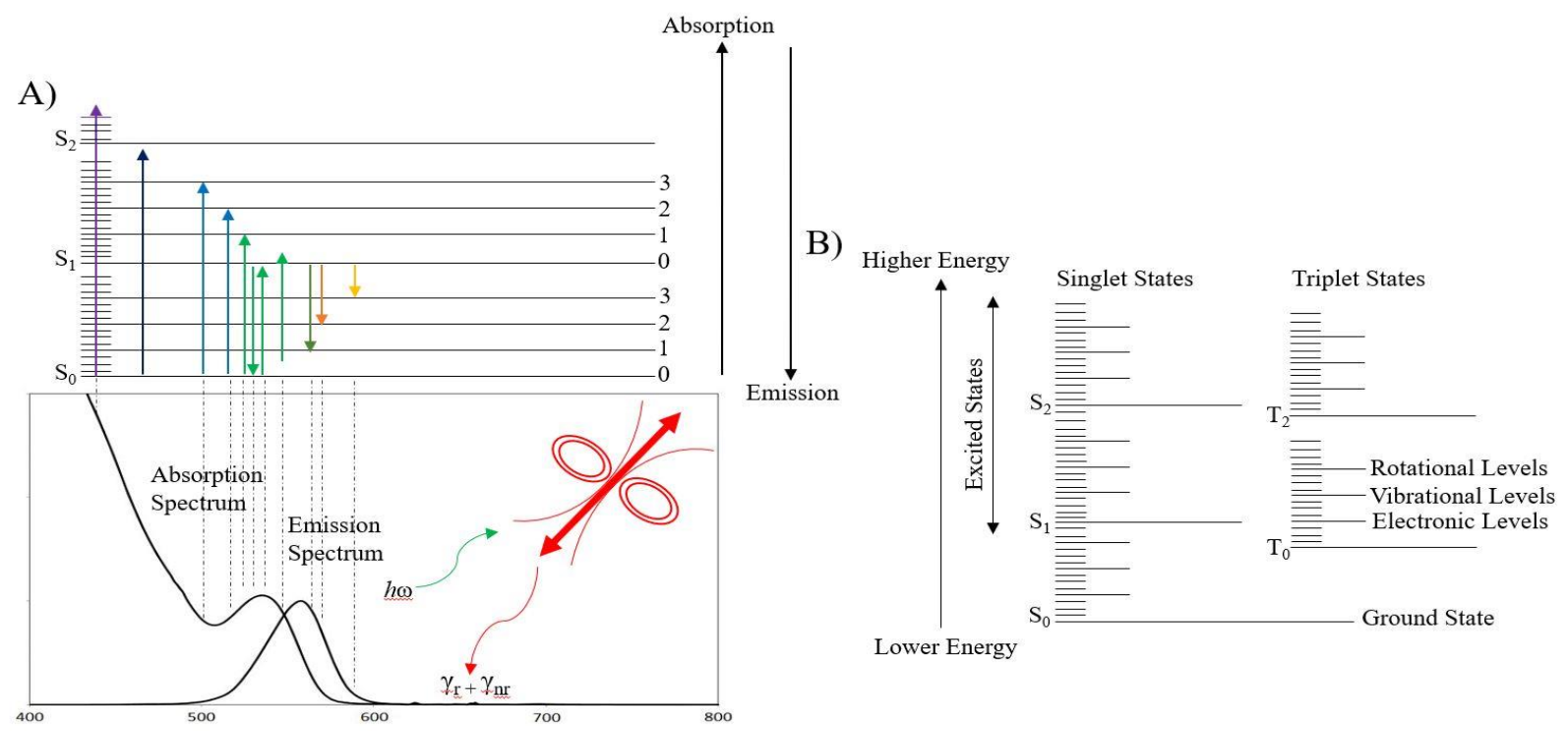

Figure 2.7 Jablonski diagram representing A) the energy levels of an excited cadmium-selenium zinc/sulfide (CdSe/ZnS) QD (as used in experimentation) of this label (a TE dipole behaving as an oscillating point charge) inlaid, and B) the electronic and vibrational energy levels resulting from excitation of the single quantum emitter and the subsequent radiative and non-radiative decay processes (adapted from [8]).

The singlet and triplet states shown in Figure 2.7 are split into vibrational and rotational sub-levels. The process of fluorescence emission generally results from a thermally equilibrated excited state (i.e., the lowest vibrational state of $S_{1}$ ) [8]. Optical absorption accounts for the largest cross-section of the excitation process and is typically on the femtosecond (fs) timescale [8]. The emission of an isolated fluorophore is characterized by its quantum yield and lifetime previously stated in (48) and (53). The relaxation of the fluorescent molecule (typically in picoseconds (ps)), involves internal conversion from higher vibrational states to the level vibrational level of the first excited state [127, 174]. Relaxation is immediately followed by radiative decay (typically in nanoseconds (ns)) with the emission of light embodying a mirror image of $\mathrm{S}_{0}$ to $\mathrm{S}_{1}$ absorption and not that of the total absorption spectrum $[8,127,174]$. Table 2.1 summarizes the timescale range for the transitional states characteristic of the fluorescence process presented in Figure 2.7. 
Table 2.1 Timescale ranges for the various processes in fluorescence [127, 174].

\begin{tabular}{|c|c|c|c|}
\hline Transition & Process & Rate Constant & Timescale (s) \\
\hline $\mathrm{S}(0) \rightarrow \mathrm{S}(1)$ or $\mathrm{S}(\mathrm{n})$ & Absorption (Excitation) & Instantaneous & $10^{-15}$ \\
\hline $\mathrm{S}(\mathrm{n}) \rightarrow \mathrm{S}(1)$ & Internal Conversion & $k(i c)$ & $10^{-14}$ to $10^{-10}$ \\
\hline $\mathrm{S}(1) \rightarrow \mathrm{S}(1)$ & Vibrational Relaxation & $k(v r)$ & $10^{-12}$ to $10^{-10}$ \\
\hline $\mathrm{S}(1) \rightarrow \mathrm{S}(0)$ & Fluorescence & $k(f)$ or $\Gamma$ & $10^{-9}$ to $10^{-7}$ \\
\hline $\mathrm{S}(1) \rightarrow \mathrm{T}(1)$ & Intersystem Crossing & $k(p T)$ & $10^{-10}$ to $10^{-8}$ \\
\hline $\mathrm{S}(1) \rightarrow \mathrm{S}(0)$ & Non-Radiative Relaxation/Quenching & $k(n r), k(q)$ & $10^{-7}$ to $10^{-5}$ \\
\hline $\mathrm{T}(1) \rightarrow \mathrm{S}(0)$ & Phosphorescence & $k(p)$ & $10^{-3}$ to 100 \\
\hline $\mathrm{T}(1) \rightarrow \mathrm{S}(0)$ & Non-Radiative Relaxation/Quenching & $k(n r), k(q T)$ & $10^{-3}$ to 100 \\
\hline
\end{tabular}

As stated earlier, single-molecule detection and imaging is possible due to highly sensitive nature of fluorescence. The coupling of fluorophores with plasmons on a metal surface allows for the amplification of the intensity of detected fluorescence. Fluorescence emission intensity can be enhanced through three main avenues: 1) increasing the excitation rate via plasmon-enhanced field intensity at the absorption wavelength of the fluorophore, 2) enhancing the quantum yield of the fluorophore, and 3) a higher directionality of plasmon-coupled emission at the emission wavelength of the fluorophore $[8,41-42,62,129-133]$. It is important to note that the fluorescence enhancement factor strongly depends on the orientation of the fluorophore due to the sensitivity of LSPR to polarization [42]. Since fluorophore orientation is typically random, the enhancement for an ensemble of emitters is averaged across all possible orientations of the absorption and emission dipole moments $\left(\mu_{a b}\right.$ and $\mu_{e m}$ ), respectively. Additionally, PEF amplification is a highly surfacesensitive mechanism only occurring at distances below the surface plasmon probing depth $\left(L_{p}\right)$ [42, 62]. PEF can therefore provide a means for better distinguishing between the specific fluorescence signal from background noise that originates from bulk effects like auto-fluorescence and/or scattering [8, 42]. Common fluorophores employed in PEF are organic dye molecules characterized by aromatic rings or conjugated carbon chains; however, QDs have also seen extensive study in PEF experimentation [66, 79, 114-116, 118, 127, 174, 181-185]. Fluorescence emission quantum yield is defined as the following:

$\phi_{\text {em }}=\frac{\# \text { photons emitted }}{\# \text { photons absorbed }}$ 
Fluorescent labels, such as rhodamines, display some of the highest emission intensities due to their incredibly high, near unity quantum yields [42, 114, 174, 183-185]. The rate equation governing the rate at which these emitters go from their ground states to higher energy excited states is shown below:

$\frac{d n_{s_{1}}}{d t}=k_{A} n_{s_{0}}-\left(k_{r}+k_{n r}\right) n_{s_{1}}$

For an emitter concentration at constant optical excitation:

$\frac{d n_{s_{1}}}{d t}=0$

Combing (57) and (58) results in the expression for the number of emitters in an excited state:

$n_{s_{1}}=\frac{n_{s_{0}} k_{A}}{\left(k_{r}+k_{n r}\right)}$

In equations (57)-(59), $n_{s_{1}}$ is the number of emitters at higher energy excited states for a given concentration, $\frac{d n_{s_{1}}}{d t}$ is the rate per unit time these emitters reach their excited states, $n_{s_{0}}$ is the number of emitters at grounded states at the same concentration, $k_{A}$ is the excitation rate enhancement $\left(\left|\frac{E}{E_{0}}\right|^{2}\right), k_{r}$ is the radiative decay rate of the emitters, and $k_{n r}$ is the non-radiative decay rate of the emitters [114]. 


\subsubsection{Fluorescence Quenching}

Fluorophore emission can be directly influenced by the interaction of a fluorophore with other fluorescent or non-fluorescent molecules. These intramolecular interactions can quench or prevent emitted fluorescence from the excited fluorophore [15, 18, 72, 114, 127, 183]. Fluorescence quenching is often used to determine the activation status of proteins or identify gene expression that can be designed for introduction into, or removal from, a particular biological response [183]. Methods of fluorescent quenching include FRET, collision quenching, and contact quenching. In the case of FRET, incident excitation light causes a fluorophore to reach an excited state resulting in the release of a photon (i.e., a "donor" molecule) [183]. Another fluorophore or a non-fluorescent molecule is placed in proximity to the donor fluorophore to absorb the emitted photon (i.e., an "acceptor" molecule) effectively quenching, or stifling, the emitted light in the case of non-fluorescent molecules [183]. Using a fluorophore to quench the photonic emission of another fluorophore is often used to detect the quenched state of the donor fluorophore whereby, instead of detecting fluorescence at a known wavelength in response to the excitation of the donor fluorophore, photonic emission of the quenched fluorophore would occur at the wavelength of the quenching fluorophore [183]. Figure 2.8 shows the FRET spectral overlap and with the types of quenching visualized in Figure 2.9.

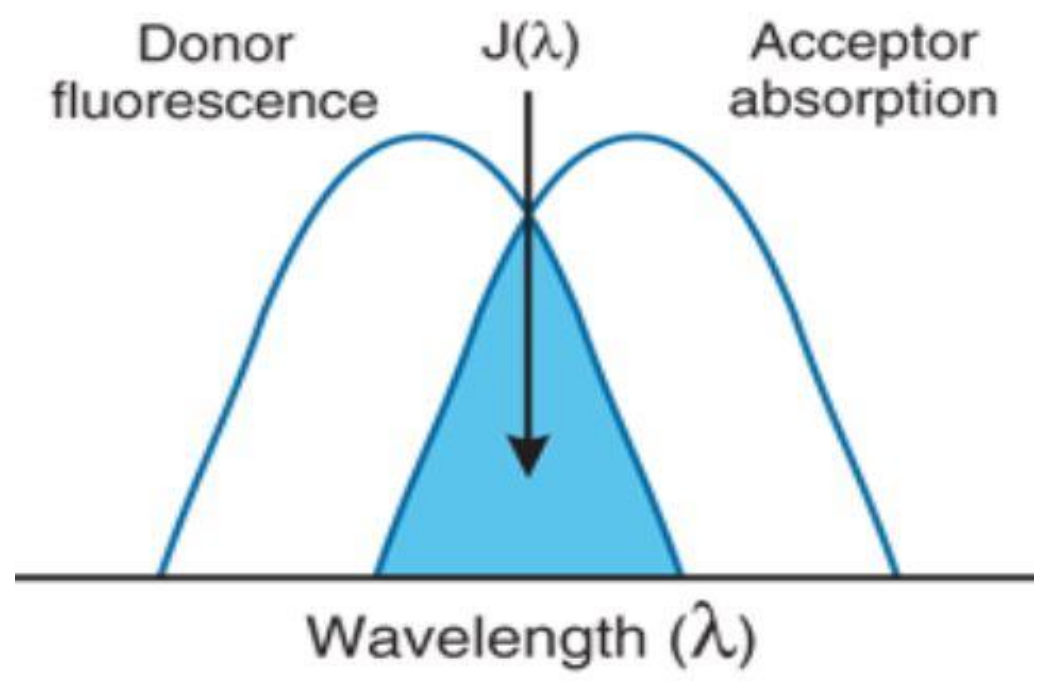

Figure 2.8 FRET spectral overlap [183]. 


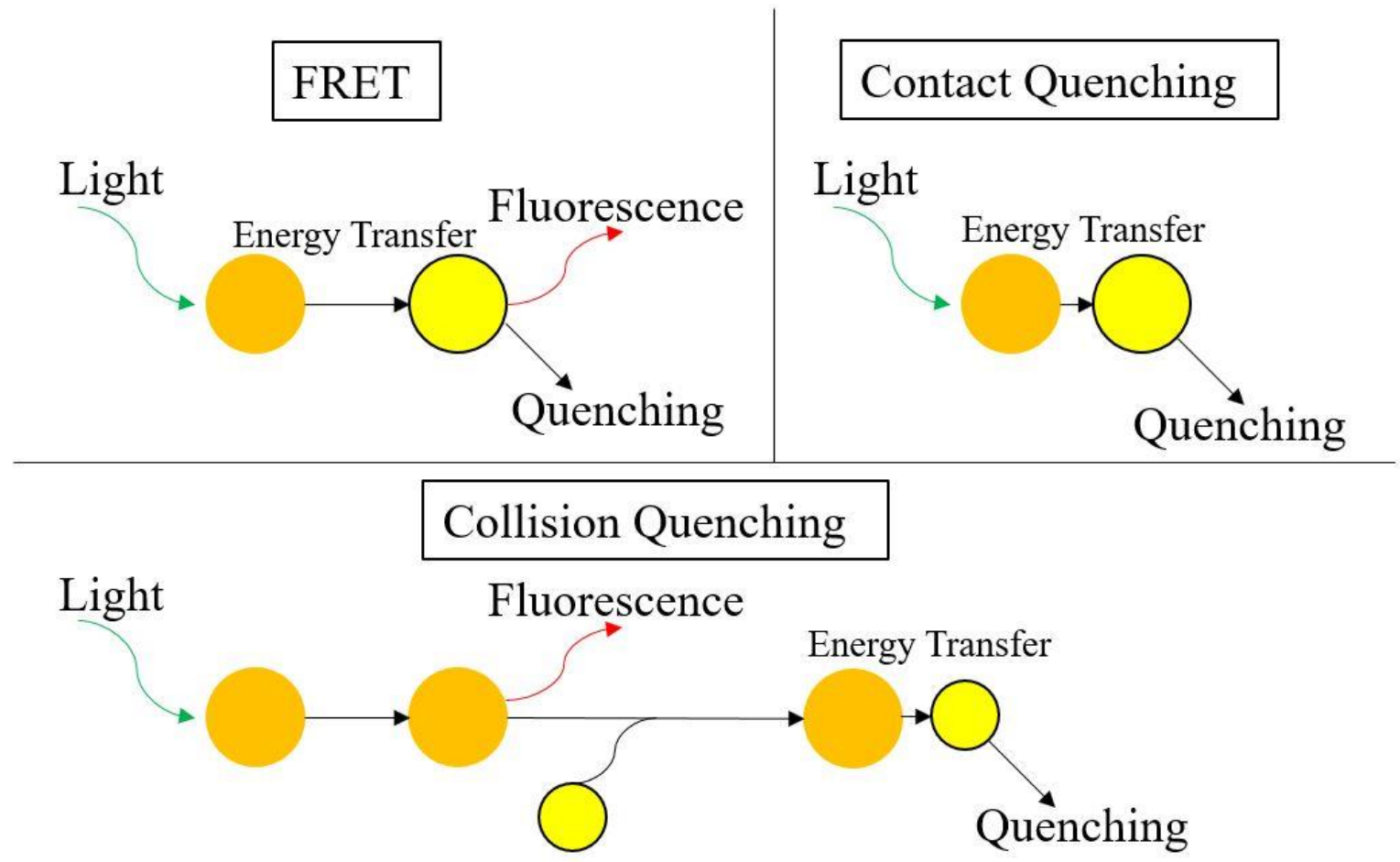

Figure 2.9 Types of fluorescence quenching (adapted from [183]).

Fluorescence quenching is directly related with the distance-dependent decay rate of an emitter and occurs when an excited quantum emitter transfers its energy to a metal or dielectric surface (typically when $\mathrm{d}<15 \mathrm{~nm}$ ) $[15,18,72,114,127,183]$. Quenching, therefore, is simply the reduction in the quantum yield of an emitter exacerbated by the non-radiative energy transfer from the emitter to a nearby NP preventing radiative decay, or emission $[15,127]$. The signature of the EM origin of the plasmonic component, this metal-molecule distance dependence has been both demonstrated and explored throughout PEF biosensor literature by many research groups [15, 17-18, 66, 72]. A continuous transition from fluorescence enhancement to quenching is observed by varying the molecule-NP distance. One of the main ways to overcome the real issue of fluorescence quenching is to deposit a $\mathrm{SiO}_{\mathrm{x}}$ spacer layer atop the plasmonic nanostructure(s) introducing separation between the emitter and the metal surface. It has been shown that, when spacer layer thickness increases from $d=0$, luminescence intensity of the emitter is observed [15, 18, 127, 174]. The extent of quenching depends on the nature of the quencher molecule (fluorophore or non-fluorophore), the type of interaction, and the wavelength of energy emitted by the fluorophore. 
Amplifying the fluorescence signal for detection requires functionalized sensors containing biomolecular recognition elements (BREs) capable of capturing specific analytes from solution $[42,179-180]$.

\subsubsection{PEF Biosensor Functionalization}

Since surface chemistries have been extensively subjected to reviews in [179] and [180], the focus of this subsection is on bio-interfacial systems adopted for local and selective attachment of analytes at plasmonic hotspots (i.e., LSP regions) [12, 42, 51, 61, 68, 82, 111, 134, 138, 150151]. Local functionalization of these plasmonic hotspots is favorable because of the high fluorescence signal is associated with the binding event. However, there is an accompanied lower concentration, or average density, of BREs present on the surface of the sensor leading to a lower probability of analyte capture [42]. These conflicting effects inherent in PEF biosensor technologies can stymie their overall sensitivity [8, 41-42]. A popular class of materials for use in biosensing applications are self-assembled monolayers (SAMs) due to their ability to tailor transducer/liquid interfacial properties [42, 178-180]. Alkanethiol SAMs reliably conjugate analytes to the surface of a noble metal through amine coupling, his-tag, and biotin-streptavidin interactions $[42,178-180]$. Silane-based surface chemistries are preferred for use with oxide layers (i.e., spacer films) that provide for distance control between the fluorophore and metal surface [15, 18, 127, 174]. S-layer protein SAMs have been employed for modifying the surface of plasmonic biosensors incorporating specific fusion proteins bearing biotin tag- or immunoglobulin (IgG)reacting functional groups [161-163]. Metallic surface functionalization can also exploit natural or synthetic polymers that, when attached to the metal surface, provide an open 3D structure accommodating larger quantities of biomolecules compared to 2D SAM systems [42, 179]. Precise attachment of BREs to plasmonic hotspots is crucial for witnessing the large fluorescence signal amplification endowed by PEF on metallic nanostructures since only the molecular binding events occurring in plasmonic hotspots are capable of producing a strongly amplified fluorescence signal $[4-10,12-13,42,66,110,146-148,158-171,178]$.

In PEF biosensors the measured sensor(s) signal is calibrated against the concentration of a target analyte being analyzed in a sample $\left(c_{\alpha}\right)$. In the case of fluorescence-based heterogenous assays, the measured fluorescence signal $(F)$ is proportional to the product of the enhancement factor and number of molecules captured on specific sensing spots [42]. 
The relationship between the number of captured molecules and depends on $c_{\alpha}$ depends on the means analyte transfer from sample to surface, the density of the BREs $\left(c_{\beta}\right)$, dissociation affinity binding constant $\left(K_{d}\right)$, and the reaction time. Via the Langmuir isotherm, the equation describing the fluorescence signal is:

$F \propto E F \propto \cdot S \frac{c_{\alpha}}{\frac{K_{d}}{\xi c_{\beta}}+\frac{S}{V}}$

Equation (60) holds for analyte concentrations smaller than the dissociation affinity binding constant $\left(c_{\alpha} \ll<K_{d}\right)$ and surface reaction at equilibrium [42]. $V$ represents the volume of the sample analyzed with $c_{\alpha}, S$ is the surface area of the sensing spot, and $\xi$ is the fraction of the total surface area occupied by plasmonic hotspots functionalized by $c_{\beta}$. For a larger $V$ and small $S$, the response of the sensor is proportional to $E F \xi S c_{\beta} c_{\alpha}$. The effect of strong PEF amplification in this limit at sparsely distributed plasmonic hotspots will not yield a substantial improvement in detection sensitivity due to the fact that large enhancement factors are typically associated with the strong EM confinement occurring for low $\xi$ [42]. Equation (60) indicates that PEF is highly favorable on the local functionalized hotspots for small $V$ when high affinity BREs are present. For the case where $c_{\beta} S \gg K_{d} V$ [42], equation (60) translates to a situation where nearly all molecules present are captured at the plasmonic hotspots and all contribute to an amplified fluorescence signal:

$F \propto E F V c_{\alpha}$

Tables 2.2-2.4 summarize select biological and chemical compounds used in PEF biosensing including information on analysis time, analyzed matrix, assay format, and LOD.

Table 2.2 Select model analytes analyzed in PEF including analysis time, analyzed matrix, assay format, and LOD.

\begin{tabular}{|c|c|c|c|c|c|}
\hline Model Analytes (SP Mode) & Matrix & LOD & Detection Time & Assay Type & Reference \\
\hline DNA (SPP) & Buffer & $30 \mathrm{pM}$ & $10 \mathrm{~min}$ & Direct & {$[156]$} \\
\hline DNA (SPP) & Buffer & $1.57 \mathrm{pM}$ & $30 \mathrm{~min}$ & Direct & {$[157]$} \\
\hline RNA (LSP) & Buffer & $25 \mathrm{pM}$ & $30 \mathrm{~min}$ & Sandwich & {$[158]$} \\
\hline Streptavidin (SPP) & Buffer & $50 \mathrm{pM}$ & $10 \mathrm{~min}$ & Direct & {$[160]$} \\
\hline Human IgG (SPP) & Buffer & $1 \mathrm{pg} / \mathrm{ml}(6.7 \mathrm{fM})$ & $1 \mathrm{hr}$ & Sandwich & {$[161]$} \\
\hline Human IgG (LSP) & Buffer & $86 \mathrm{pg} / \mathrm{ml}(0.57 \mathrm{pM})$ & $1 \mathrm{hr}$ & Direct & {$[163]$} \\
\hline Human IgG (LSP) & Buffer & $0.3 \mathrm{fM}$ & $1 \mathrm{hr}$ & & \\
\hline
\end{tabular}


Table 2.3 Select biomarkers analyzed in PEF biosensing including information on analysis time, analyzed matrix, assay format, and LOD.

\begin{tabular}{|c|c|c|c|c|c|}
\hline Biomarkers (SP Mode) & Matrix & LOD & $\begin{array}{c}\text { Detection } \\
\text { Time }\end{array}$ & Assay Type & Reference \\
\hline f-PSA (LRSPP) & Buffer/Serum & $34 / 330 \mathrm{pM}$ & $35 \mathrm{~min}$ & Sandwich & [164] \\
\hline Total PSA (LSP) & Buffer/Serum & $0.4 / 1.8 \mathrm{pg} / \mathrm{ml}(12 / 52 \mathrm{fM})$ & $1 \mathrm{hr}$ & Sandwich & {$[165]$} \\
\hline TNF- $\alpha$ (LSP) & N/A & $3 \mathrm{pM}$ & $2 \mathrm{hr}$ & Sandwich & {$[166]$} \\
\hline Troponin I (LSP) & Buffer/Blood & $5 / 50 \mathrm{pg} / \mathrm{ml}(0.22 / 4.3 \mathrm{pM})$ & $1 \mathrm{~min}$ & Sandwich & {$[167]$} \\
\hline C-Reactive Protein (SPP) & Buffer/Serum & $16 / 26 \mathrm{ng} / \mathrm{ml}(0.15 / 0.25 \mathrm{nM})$ & $30 \mathrm{~min}$ & Sandwich & {$[168]$} \\
\hline UL16-Binding Protein 2 (LSP) & Serum & $18 \mathrm{pg} / \mathrm{ml}(0.75 \mathrm{fM})$ & $4.3 \mathrm{hr}$ & Sandwich & [169] \\
\hline
\end{tabular}

Table 2.4 Select pathogens and toxins analyzed in PEF biosensing including information on analysis time, analyzed matrix, assay format, and

\begin{tabular}{|c|c|c|c|c|c|}
\hline Pathogens and Toxins (SP Mode) & Matrix & LOD & $\begin{array}{c}\text { Detection } \\
\text { Time }\end{array}$ & Assay Type & Reference \\
\hline Aflatoxin M1 (LRSPP) & Buffer/Milk & $0.6 \mathrm{pg} / \mathrm{ml}(1.8 \mathrm{pM})$ & $53 \mathrm{~min}$ & Inhibition & {$[170]$} \\
\hline E. coli 157 (LRSSP) & Buffer & $6 \mathrm{cfu} / \mathrm{ml}$ & $20 \min$ & Sandwich & {$[171]$} \\
\hline SARS-CoV (LSP) & Buffer & $13.9 \mathrm{pg} / \mathrm{ml}$ & N/A & Sandwich & {$[172]$} \\
\hline S-OIV (LSP) & Buffer/Serum & $0.1 / 1 \mathrm{pg} / \mathrm{ml}$ & N/A & Sandwich & {$[173]$} \\
\hline Anthrax Protective Antigen (LSP) & Buffer & $0.1 \mathrm{pg} / \mathrm{ml}$ & $40 \mathrm{~min}$ & Inhibition & {$[178]$} \\
\hline
\end{tabular}

Controlling the emission rate of the fluorescent label is vital in fluorescence emission enhancement and will be discussed in Section 2.1.5.

\subsubsection{Purcell Factor}

In 1946, E. M. Purcell found that the spontaneous emission rate of a single emitter (e.g., an atom, molecule, or QD) can be amplified through the resonant coupling of an emitter to its surrounding environment (i.e., the Purcell Effect). The lifetime of an excited emitter is governed by both the radiative decay rate relating to photon emission as well as the non-radiative decay rate relating to energy dissipation into the surrounding environment [15, 62, 74-75, 79, 109-110, 123]. The spontaneous emission rate of a single fluorescent emitter can be significantly enhanced when coupled to metallic NPs, multilayer or surface metal films, PhCs, and resonant cavities [62, 74-75, 79, 109-110]. For a system to be effectively coupled to an EM resonator, the spontaneous emission probability is increased over its bulk value yielding a recombination time reduced by the following factor:

$F_{P}=\frac{3}{4 \pi^{2}}\left(\frac{Q}{V}\right)\left(\frac{\lambda}{\eta}\right)^{3}$ 
The parameters contained in (62) are the quality factor $(Q)$, the mode volume $(V)$, and the wavelength in the material $(\lambda)[62,74-75,79]$. The quality factor of the cavity mode is defined as:

$Q=\frac{f_{c}}{\Delta \mathrm{f}}=\frac{2 \pi f_{c} E}{\rho}=\frac{\tau \omega_{0}}{2}$

$f_{c}$ is the resonant frequency of the cavity mode and $\Delta \mathrm{f}$ is the full-width half-maximum, or bandwidth of the frequency response, $E$ is the total energy stored in the cavity mode, $\tau$ is the average lifetime of a resonant photon in the cavity mode (directly proportional to $Q$ ), and the expression for power dissipation $\rho=-d E / d t$. $Q$ pertains to the ratio of energy stored to the energy dissipation per optical cycle. Additionally, $Q$ represents the maximum amount of energy stored vs. power loss at an angular frequency $\left(\omega_{0}\right)$ at which stored energy and power loss are measured. Note that a low $Q$ delineates an overdamped system with a high $Q$ delineating an underdamped system $[79,109]$. Furthermore, $\tau$ is directly proportional to $Q$. Expanding on (62):

$F=\frac{R_{s p, c a v}}{R_{\text {bulk }}}$

$R_{s p, c a v}$ is the spontaneous emission rate into the resonant cavity mode per unit time and $R_{b u l k}$ is the total spontaneous rate per unit time in the bulk medium absent of the resonant cavity [79]. The Purcell factor shown in (62), in the case of an ideally matched single quantum emitter, is equal to the Purcell factor shown in (64) [79].

The spontaneous emission coupling factor $(\beta)$ is explicitly defined as the fraction of photonic emission coupled to the cavity mode:

$\beta=\frac{R_{s p, c a v}}{R_{s p, c a v}+R_{l}}$

Above, $R_{l}$ is the emission rate per unit time into other modes [79]. It can be observed that that $\beta$ can be enhanced substantially by ensuring $R_{l}$ is suppressed, even in the case where $R_{s p, c a v}=R_{b u l k}$ (without any Purcell enhancmenet). 
The photonic emission rate for a homogenously broadened two-level atom in a resonant cavity can be directly derived from Fermi's golden rule:

$R_{e m}=\frac{2 \pi}{\hbar^{2}} \int_{0}^{\infty}|\langle f|H| i\rangle|^{2} \rho(\omega) L(\omega) d \omega$

$\rho(\omega)$ is the LDOS per unit of angular frequency $(\omega), L(\omega)$ is the homogeneous broadening line shape, $H$ is the atom-field Hamiltonian, $i$ represents the initial transition states, and $f$ represents the final transition states [79]. The cavity line shapes for the cavity and emitter are both typically Lorentzian. Considering an electric dipole transition and a single cavity mode taking a density matrix approach with artificial discretization of the LDOS yields the following matrix element:

$$
\begin{aligned}
& |\langle f|H| i\rangle|=E_{0}\left(\vec{r}_{e m}\right) \hat{e} \cdot \vec{d}_{i f} \sqrt{N_{p h}+1} \\
& E_{0}\left(\vec{r}_{e m}\right)=\sqrt{\left(\hbar \omega / 2 \varepsilon_{0} \varepsilon_{r a} V\right)} e\left(\vec{r}_{e m}\right)
\end{aligned}
$$

$N_{p h}$ is the number of photons within the mode, $E_{0}\left(\vec{r}_{e m}\right)$ is the field magnitude per photon at the emitter's position $\left(\vec{r}_{e m}\right), \hat{e}$ is a unit vector inidicating polarization, here again $\varepsilon_{0}$ is the free-space permittivity, $\varepsilon_{r a}$ is the relative dielectric constant in the active material, and $V$, again, is the mode volume [79]. Equation (67) denotes stimulated emission while equation (62) denotes spontaneous emission. The dimension-less mode function $(e(\vec{r}))$ is then normalized to $|e(\vec{r})|_{\max }=1$. For an optimally positioned point-like emitter:

$|\langle f|H| i\rangle|=\sqrt{\frac{\hbar \omega}{2 \varepsilon_{0} \varepsilon_{r a} V}} \hat{e} \cdot \vec{d}_{i f} \sqrt{N_{p h}+1}$

Defining the atomic dipole moment:

$$
\vec{d}_{i f}=\left|\left\langle\psi_{i}|\vec{d}| \psi_{f}\right\rangle\right|
$$

The parameter $\vec{d}$ is the dipole operator, $\psi_{i}$ is the upper level wavefunction of the atom, and $\psi_{f}$ is the lower level wavefunction of the atom [79]. 
The mode volume $(V)$ is designated by the energy normalization condition of the field per photon $\left(E_{0}\right)$ and, in the case of a non-dispersive dielectric cavity, produces:

$\int 2 \varepsilon(\vec{r})\left|E_{0}(\vec{r})\right|^{2} d^{3} \vec{r}=\hbar \omega$

Therefore, the expression for the cavity mode volume becomes:

$V=\frac{\int_{V} \varepsilon(r)|E(r)|^{2} d^{3} r}{\max \left(\varepsilon(r)|E(r)|^{2}\right)}$

$\varepsilon(r)$ is the electric permittivity of the material at position $r$ [79, 124]. Based on the discussion presented in [124], (72) assumes the case of an ideally oriented and positioned dipole yielding the highest Purcell factor. The Purcell factor in (62) considers a cavity whose fundamental mode is in resonance with the transition frequency of a dipole in alignment with the polarization of the cavity mode at a position of maximum field [74]. Consequently, experimental observation of the Purcell effect is relatively challenging in the field of optics because a significant emission increase requires specialized optical resonators capable of confining and storing light comparable to the wavelength [79]. Due to the wider cavity resonance line widths (i.e., a wider frequency response or spectrum) of the plasmonic lattice geometries modeled using FDTD (Chapter 3) compared to the line width of applicable fluorescent labels, the case championed in Figure 2.10 will now be explored. 


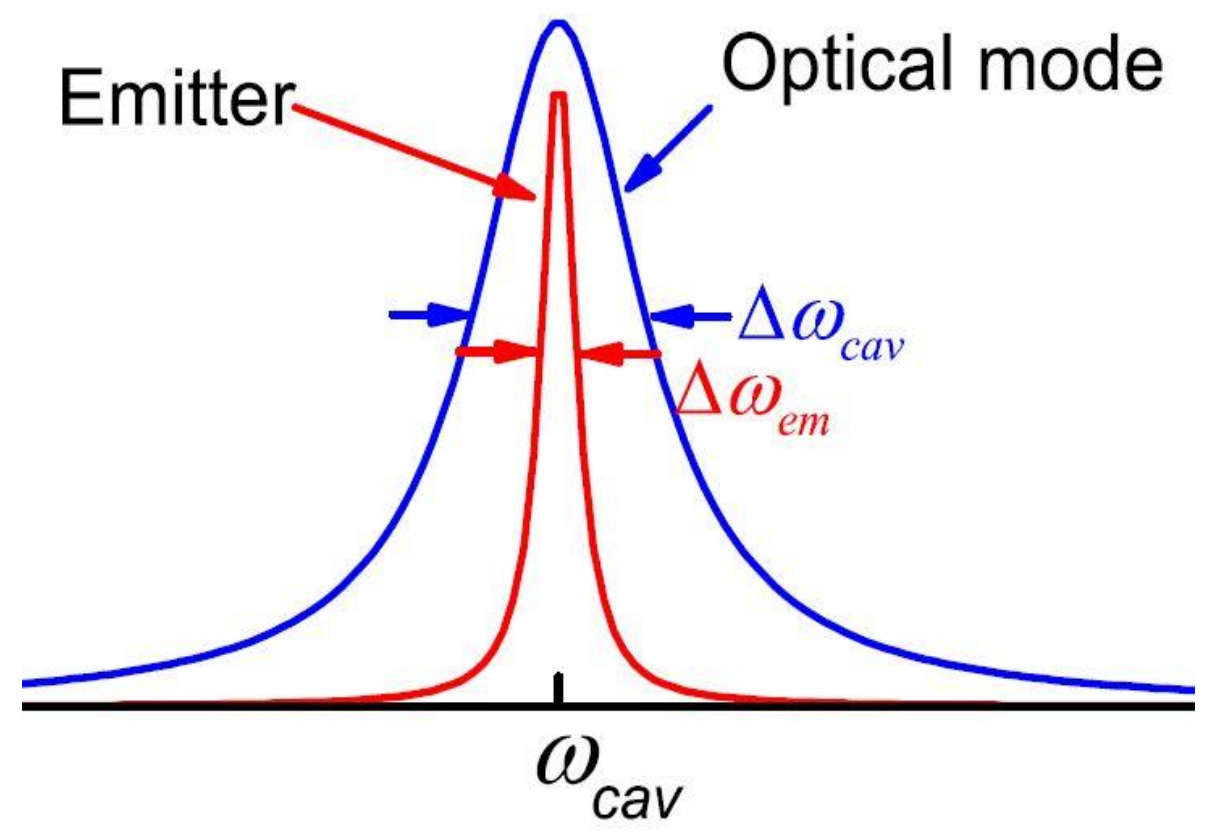

Figure 2.10 Relationship between cavity mode resonance and the transition spectrum of the emitter in the case where the line width of the cavity mode is broader than the transition line width of the emitter [79].

With a uniform dielectric constant throughout the entirety of the cavity:

$V=\int|e(\vec{r})|^{2} d^{3} \vec{r}$

Spontaneous and stimulated emission processes will be analyzed using equations (66), (67), and (69) considering the case where $\rho(\omega)$ is nonzero within a narrow line width (i.e., a single optical mode in the gain spectrum depicted in Figure 2.10) where the matrix element is frequencyindependent and can therefore be removed from equation (66) $[79,124]$. An average dipole moment $\left(d_{i f}=\left\langle\hat{e} \cdot \vec{d}_{i f}\right\rangle\right)$ is assumed for emitters possessing dipole moments oriented along different directions [79]. The photon creation rate via spontaneous and stimulated emission in the cavity mode for a point-like optimally positioned emitter, maintaining the assumption $\left|e\left(\vec{r}_{e m}\right)\right|=$ 1, becomes:

$R_{s p, s t, c a v}=\frac{\pi}{\hbar \varepsilon_{0} \varepsilon_{r a}} \frac{d_{i f}^{2}}{V}\left(N_{p h}+1\right) \int_{0}^{\infty} \rho(\omega) L(\omega) d \omega$ 
For a narrow emitter $\left(\Delta \omega_{e m} \ll \Delta \omega_{\text {cav }}\right.$ pictured in Figure 2.10) and assuming the emitter is in resonance with the cavity mode $\left(\omega=\omega_{\text {cav }}\right)$, the Lorentzian optical mode densities at the emission frequency:

$\rho\left(\omega_{e m}\right) \cong \frac{2}{\pi \Delta \omega_{c a v}}$

Approximating the spontaneous and stimulated emission rates in the cavity mode (i.e., $L(\omega)$ as a Dirac delta function):

$R_{s p, s t, c a v} \cong \frac{2}{\hbar \varepsilon_{0} \varepsilon_{r a}} d_{i f}^{2} \frac{Q}{V}\left(N_{p h}+1\right)$

As previously mentioned in (63), $Q=\omega_{\text {cav }} / \Delta \omega_{c a v}$, and $\omega_{c a v}$ is the resonant frequency of the cavity. Dividing equation (76) by the bulk emission $\left(R_{b u l k}\right)$ for the case of spontaneous emission $\left(N_{p h}=0\right)$, the originally postulated Purcell factor in (62) is obtained [74, 79]. The factor $Q / V$ holds true only in specific cases where the transition frequency of the emitter is narrow [79]. The photon creation rate via spontaneous and stimulated emission of a single QD operating at cryogenic temperature directly depends on the ratio $Q / V$. This ratio essentially states that the spontaneous emission rate can be Purcell-enhanced [74, 79, 109]. A prime example of the Purcell-enhanced photonic emission of a single emitter has been exemplified in the Fabry-Pérot interferometer and will be probed in Section 3.3.

\subsubsection{Metallic Nanostructures}

The subsequent subsections discuss the Purcell factors (where appropriate) and plasmon resonances of the following metallic nanostructures: thin metal films, single metal NPs, and NP arrays. FDTD simulation analysis of metal NP arrays will be presented in the latter portion of Chapter 3. Table 2.5 compares field intensity enhancement for small distances from select plasmon-supporting metallic surfaces $(d \sim 10-20 \mathrm{~nm})$ as well as the associated fabrication techniques and resonance conditions, respectively. 
Despite the stronger field intensity enhancements obtainable using silver, especially for wavelengths less than $600 \mathrm{~nm}$ [42], this thesis will delve into the slightly weaker plasmonenhanced field intensities of gold nanostructures due to gold's biocompatibility and chemically stability.

Table 2.5 Comparison of respective field intensity enhancements, fabrication techniques, and resonance conditions of select SPP- and LSPsupporting metal nanostructures [42], [131], [133-140].

\begin{tabular}{|c|c|c|c|}
\hline Plasmonic Mode and Structure & $\begin{array}{c}\text { Intensity } \\
\text { Enhancement } \\
\left(|\mathbf{E}|^{2} /\left|\mathbf{E}_{0}\right|^{2}\right) \\
\end{array}$ & Wavelength & Reference \\
\hline SPP: Flat and Corrugated Au Films & $\sim 90$ & $\lambda=630 \mathrm{~nm}$ & [42] \\
\hline SPP: Flat and Corrugated Au Films & $\sim 45$ & $\lambda=630 \mathrm{~nm}$ & [42] \\
\hline LSPR: Au Nanoshell and Spherical NPs & $\begin{array}{l}\sim 10 \\
181\end{array}$ & $\begin{array}{l}\lambda=617 \mathrm{~nm} \\
\lambda=780 \mathrm{~nm}\end{array}$ & [42] \\
\hline LSPR: Au NP Dimers & $\begin{array}{l}\sim 350 \\
\sim 100\end{array}$ & $\begin{array}{l}\lambda=780 \mathrm{~nm} \\
\lambda=630 \mathrm{~nm}\end{array}$ & [42] \\
\hline $\begin{array}{l}\text { SPP and LSPR: Au Nanodisk and Nanohole } \\
\text { Arrays }\end{array}$ & $\sim 10$ & $\lambda \sim 630 \mathrm{~nm}$ & [42] \\
\hline LSPR: Ag Bow Tie NP Arrays & $\sim 100$ & $\lambda \sim 780 \mathrm{~nm}$ & {$[42]$} \\
\hline $\begin{array}{c}\text { SPP and LSPR: Concentric Gratings with } \\
\text { Nanoholes }\end{array}$ & $\sim 10$ & $\lambda \sim 633 \mathrm{~nm}$ & [42] \\
\hline $\begin{array}{c}\text { LSPR and SPP: Diffractive Arrays of } \\
\text { Cylindrical NPs } \\
\end{array}$ & $\sim 200$ & $\lambda=630 \mathrm{~nm}-670 \mathrm{~nm}$ & [42] \\
\hline SPR: Ag Cylindrical Channel Nanopillars & 52,900 & $\lambda \sim 1,015 \mathrm{~nm}$ & [76] \\
\hline LSPR: Arrays of Au Square Nanopillars & $\sim 13.69$ & $\lambda=560 \mathrm{~nm}-565 \mathrm{~nm}$ & [240] \\
\hline LSPR: Au Nanospheres & $\sim 6.25$ & $\lambda \sim 525 \mathrm{~nm}$ & {$[241]$} \\
\hline LSPR: Au Nanostars & $\sim 36-256$ & $\lambda \sim 575 \mathrm{~nm}-750 \mathrm{~nm}$ & [241] \\
\hline LSPR: Au Nanospheres & $\sim 1,600$ & $\lambda \sim 505 \mathrm{~nm}$ & [242] \\
\hline LSPR: Au Nanostars & $\sim 133,225$ & $\lambda \sim 625 \mathrm{~nm}$ & [242] \\
\hline LSPR: Au Nanotriangles & $\sim 8,100$ & $\lambda \sim 525 \mathrm{~nm}$ & [242] \\
\hline LSPR: Au Nanotriangles & 10,000 & $\lambda \sim 649 \mathrm{~nm}$ & [243] \\
\hline LSPR: Au Nanostars & $\begin{array}{l}21,025 \\
27,225 \\
62,001 \\
\end{array}$ & $\begin{array}{l}\lambda=594 \mathrm{~nm} \\
\lambda=717 \mathrm{~nm} \\
\lambda=804 \mathrm{~nm}\end{array}$ & [244] \\
\hline LSPR: Au Nanorods & 1,000 & $\lambda=650 \mathrm{~nm}$ & [245] \\
\hline LSPR: Au Nanorods & $\begin{array}{l}324 \\
324 \\
\end{array}$ & $\begin{array}{l}\lambda=526 \mathrm{~nm} \\
\lambda=630 \mathrm{~nm}\end{array}$ & [246] \\
\hline LSPR: Au Nanotriangle & 400 & $\lambda=803 \mathrm{~nm}$ & {$[247]$} \\
\hline LSPR: Au Nanocone Arrays & $\begin{array}{l}25 \\
25 \\
25 \\
25 \\
\end{array}$ & $\begin{array}{l}\lambda=395 \mathrm{~nm} \\
\lambda=406 \mathrm{~nm} \\
\lambda=411 \mathrm{~nm} \\
\lambda=430 \mathrm{~nm}\end{array}$ & [248] \\
\hline LSPR: Au Nanospheres & 2.56 & $\lambda=520 \mathrm{~nm}$ & [249] \\
\hline LSPR: Au Nanorods & 1.44 & $\lambda=925 \mathrm{~nm}$ & [249] \\
\hline LSPR: Au Nanostars & 256 & $\lambda=720 \mathrm{~nm}$ & [249] \\
\hline
\end{tabular}

\subsubsection{Continuous Metallic Films}

SPPs on continuous metal surfaces (films) were traditionally used for enhancing the excitation field strength at the absorption wavelength of the metal $\left(\lambda_{a b}\right)$ and exploiting the resulting SP-driven emission $\left(\lambda_{e m}\right)$ [42, 70, 93-95]. SPPs have been demonstrated as effective collectors of fluorescence light (more than $50 \%$ photons) at $\lambda_{e m}$ when in close proximity to a metallic surface $[42,70,93-95$, 101-103]. 
Figure 2.11 presents a reverse Kretschmann configuration showing how SPPs can tunnel through a thin metal film into the neighboring dielectric substrate whereby the light forms a conically shaped, highly directional far-field propagation (i.e., SPCE) [42, 161].
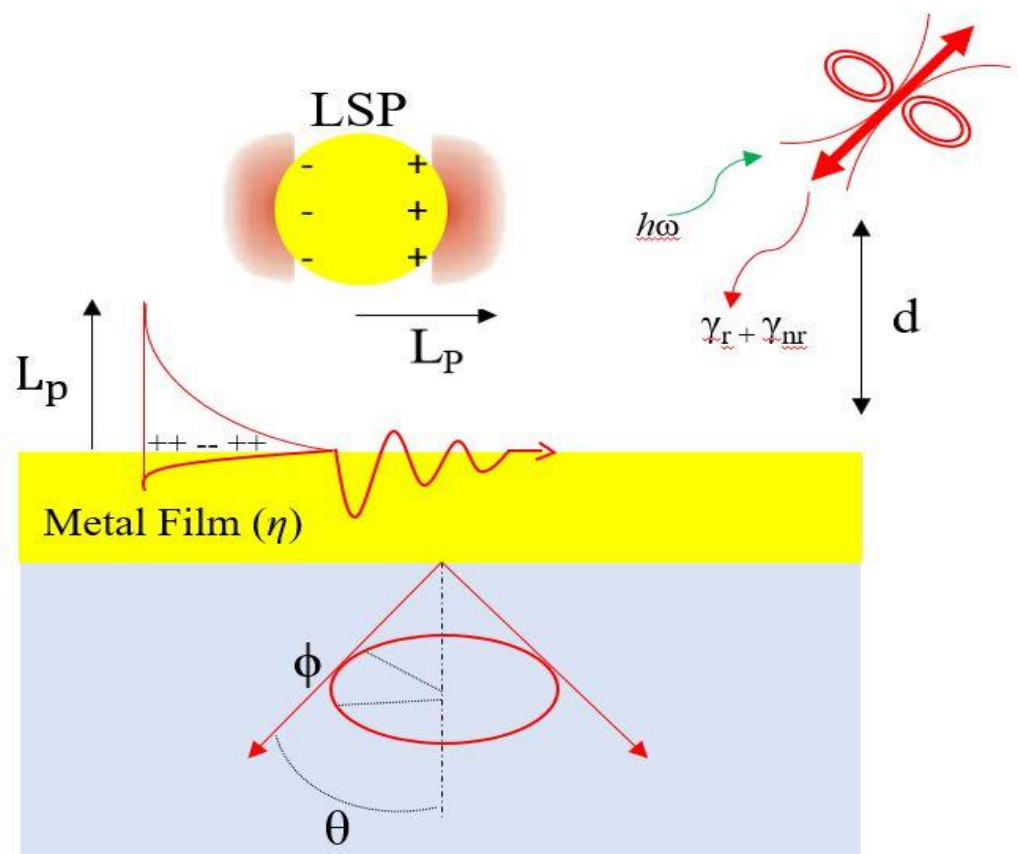

Figure 2.11 Reverse Kretschmann device architectures employ attenuated total internal reflection (ATIR) methodologies showing polar $(\theta)$ and azimuthal $(\phi)$ angles. Confined fields SPP and LSP modes coupled with a nearby fluorophore at $\lambda_{e m}$ (adapted from [42]).

This particular type of emission at $\lambda_{e m}$ can be combined with the SPP excitation at $\lambda_{a b}$ that occurs at a slightly different angle [42, 93-95, 101-103]. A thin continuous metal film supports SP modes on both of its surfaces allowing for tunability in these SP modes traveling along the surface [9395, 101-103]. These SP modes become coupled with the thickness of the metal film $\left(d_{m}\right)$ when $d_{m}$ is comparable to the approximately $10 \mathrm{~nm}$ plasmon penetration depth into the metal and when the metal is surrounded by a dielectric material(s) or medium(s) of a similar refractive index [101103]. Note that coupled symmetric and antisymmetric SPP modes are established by the spatial overlap and phase matching between SPPs [42]. Similar to SPR spectrometers, the reverse Kretschmann device architecture shown in Figure 2.11 can utilize ATIR or periodically grated metallic surfaces to extract emitted radiation [42, 93, 95, 101-103]. Grated, sub-diffractive metallic surfaces can also excite coupled SPP modes [42, 102-103]. 
Diffraction on a periodically corrugated metal surface presents an alternative methodology for simultaneous SPP-enhanced excitation at $\lambda_{a b}$ and the subsequent extraction of SPP-driven emission at $\lambda_{e m}[42,93,95,101-103]$.

Such periodicity permits the interaction between counterpropagating SPPs opening an SPP dispersion relation bandgap [42, 132, 164, 170-171]. At the edges of the bandgap, two Braggscattered surface plasmon polariton (BSSPP) modes manifest with localized field intensities present in the valleys and/or peaks of the periodic modulation [42]. To further amplify this field intensity, arrays of nanoholes can be perforated into the film allowing one to confine the SPP fields to the metal surface. These perforations act as a diffractive grating for SPP excitation simultaneously supporting laterally confined LSPs [42, 93, 95, 101-103]. Long range surface plasmon polaritons (LRSPPs) specifically pertain to SPP modes with an antisymmetric profile of the parallel $E$-field component $\left(E_{\|}\right)$with short range surface plasmon polaritons (SRSPP) relating to symmetric SPP modes [132, 164, 170-171]. The weak-natured LRSPPs are guided by the metal film and exhibit decreased Ohmic losses allowing them to propagate to longer distances [42, 93, 95, 101-103]. Additionally, their fields are capable of probing to longer distances $\left(L_{p}\right)$ from the surface of the metal [42, 101-103]. The expressions governing SPP and SPR field propagation and enhancement have been previously elaborated on in Section 2.1.1 and Section 2.1.2. Table 2.6 presents experimental fluorescence enhancement factors of certain fluorescent labels and their intrinsic quantum yields (where available) used in conjunction with continuous metallic films and the type of SP modes exploited through the use of these films, respectively.

Table 2.6 Experimental fluorescence enhancement factors of certain fluorescent labels used in conjunction with continuous metallic films, the intrinsic quantum yields of the fluorophores (where available), and the type of SP modes supported by these nanostructures, respectively.

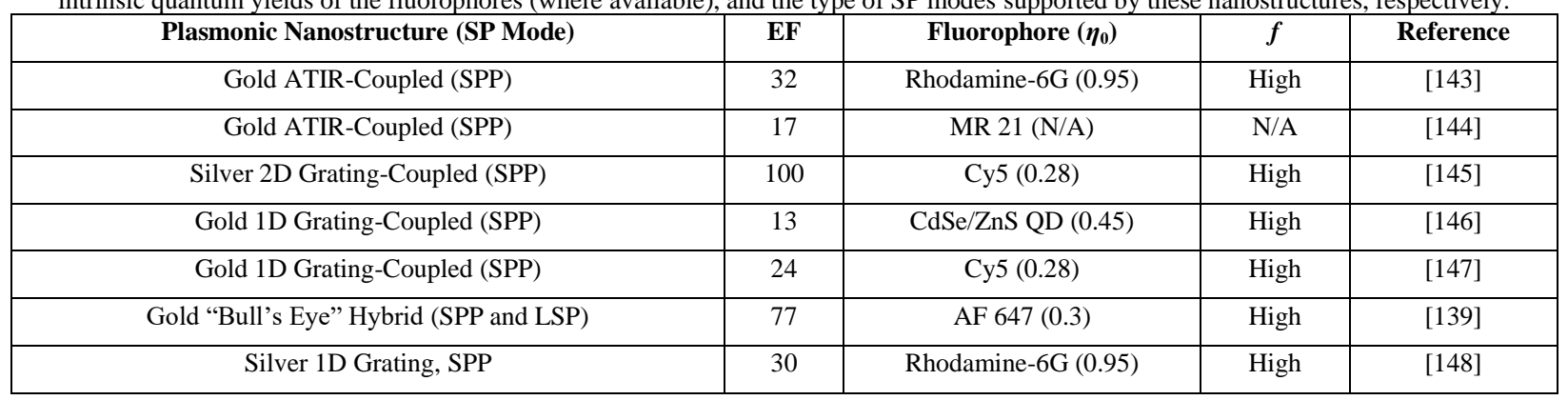




\subsubsection{Single Metallic Nanoparticles}

Arguably one of the most heavily investigated plasmonic nanostructures is the spherical metal NP $[41-43,73,75,91,95,100]$. If the diameter of the metal sphere $(D)$ is much smaller than the resonance condition, only a dipole LSP mode is supported. Consequently, the plasmonenhanced field intensity decreases as distance from the NP $(d)$ increases:

$\left|\frac{E}{E_{0}}\right|^{2} \sim \frac{D}{[0.5 D+d]^{3}}$

(77) provides an estimation of the probing depth of the LSP field that roughly scales with NP diameter $\left(L_{p} \sim D\right)$ [42]. LSPR occurs at longer wavelengths on spherical NPs possessing a dielectric core and a thin metal layer shell cap (nanoshell). The interaction between LSP modes at the inner metal-dielectric interface and the outer shell cause a red-shift in the LSPR wavelength and a higher field intensity [100-104]. Asymmetrical NPs support multiple LSP modes at varying wavelengths. For instance, elongated gold nanorods support LSP modes with a dipole moment oscillating both parallel and perpendicular to the NP axis [77-78, 100].

For small gold nanorods, the ensemble extinction has two strong peaks corresponding to the transverse and longitudinal modes of the NP, but the single-particle scattering is too weak to be seen as the extinction is dominated by absorption [75, 77-78]. However, for larger nanorods, scattering makes up a larger portion of the extinction and can be measured experimentally [77-78]. Introducing asymmetry in a single metal NP results in a significant red-shift of the LSP resonant wavelength $[77-78,100]$. The case of a single spherical metal NP placed in the vicinity of an $\mathrm{InGaN} / \mathrm{GaN}$ quantum well $(\mathrm{QW})$ as pictured in Figure 2.12 will now be explored. 


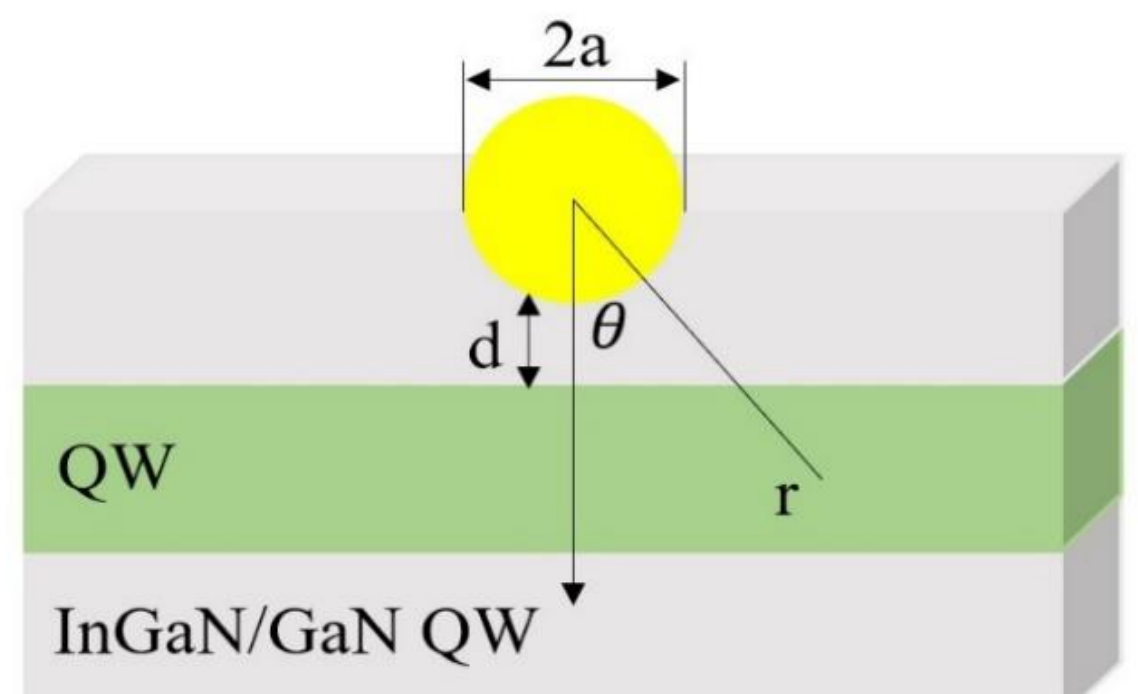

Figure 2.12 Isolated nanosphere (NS) in the vicinity of an active InGaN QW is GaN (adapted from [75]).

Determining the effective SP eigenmode volume supported by the metal NS with the $z$-axis perpendicular to the sample plane first requires one to obtain the dipole field inside and outside of the metal sphere with radius $a$ [75]:

$$
\begin{array}{ll}
E(r, \theta)=\left\{\frac{3 \varepsilon_{D}}{\varepsilon_{m}+2 \varepsilon_{D}} E_{0} \hat{z}\right\} & r<a \\
E(r, \theta)=\left\{E_{0}\left(\frac{\varepsilon_{m}-\varepsilon_{D}}{\varepsilon_{m}+2 \varepsilon_{D}}\right) \frac{a^{3}}{r^{3}}[2 \cos (\theta) \hat{r}-\sin (\theta) \hat{\theta}]\right\} & r>a
\end{array}
$$

Recalling the Drude model:

$$
\varepsilon_{M}=\frac{\omega_{p}^{2}}{\omega^{2}+j \omega \gamma}
$$

Where $\omega_{p}$ is the plasmon frequency, $\gamma$ is the metal loss, and $\varepsilon_{D}$ is the dielectric constant of the medium surrounding the metal NS. The field inside the NS remains despite the absence of the outside $z$-polarized driving $E$-field when the following condition is met:

$$
\varepsilon_{M}\left(\omega_{0}\right)+2 \varepsilon_{D}=0
$$


The expression shown in (81) details and unambiguous indication of an eigenmode oscillating at resonance:

$\omega_{0}=\frac{\omega_{p}}{\left(1+2 \varepsilon_{D}\right)^{1 / 2}}$

In the event where $r=a$, the field surrounding the surface of the NS is at its maximum [75]:

$E_{\max }=\frac{6 \varepsilon_{D} E_{0}}{\varepsilon_{M}+2 \varepsilon_{D}}$

The emission process (pictured in Figure 2.13) of a single quantum emitter is treated as a two-step process: 1) material polarization coupling into a closely confined SP eigenmode with a Purcellenhanced radiative rate $\left(1 / \tau_{\text {rad }}\right)$ that competes with the non-radiative decay rate of the emitter $\left(1 / \tau_{\text {nrad }}\right)$, and 2$)$ energy coupling from the SP mode into the radiation continuum rate $\left(\gamma_{\mathrm{rad}}\right)$ [75]. This coupling competes with the non-radiative loss $\left(\gamma_{n r a d}\right)$ because of the absorption in the metal $[42,75]$, and can be defined as:

$\eta_{p r}=\frac{\gamma_{\text {rad }}}{\left(\gamma_{\text {rad }}+\gamma_{\text {nrad }}\right)}$ 

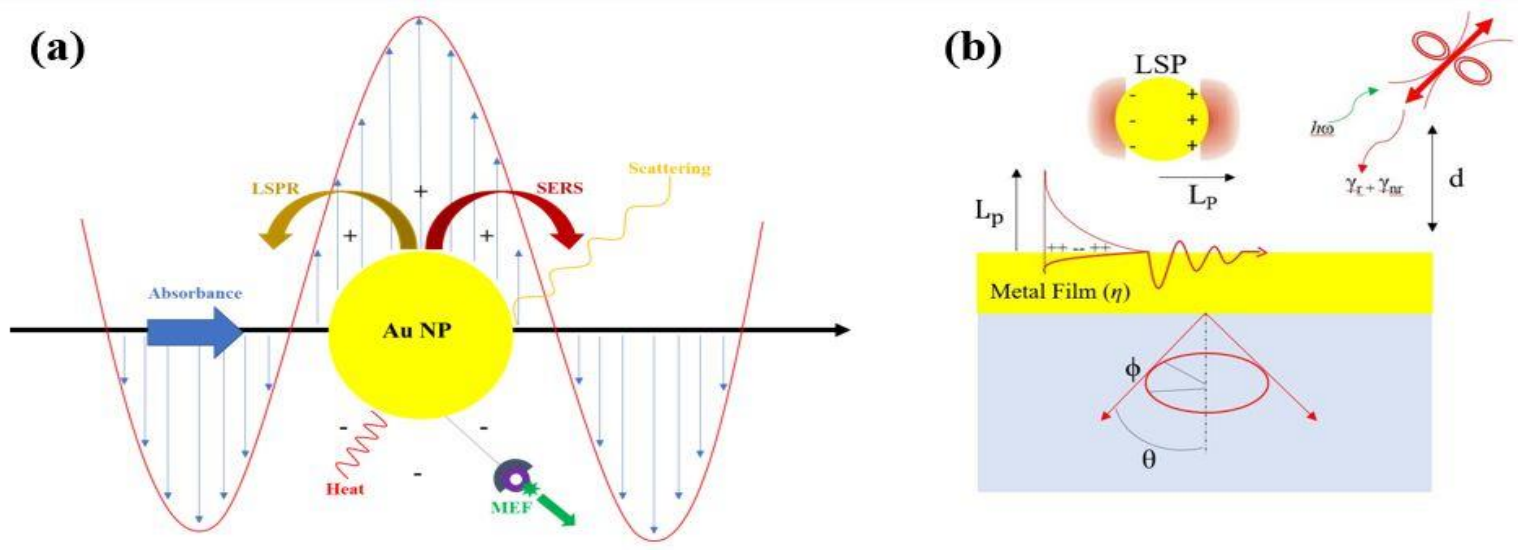

(c) Emission Process

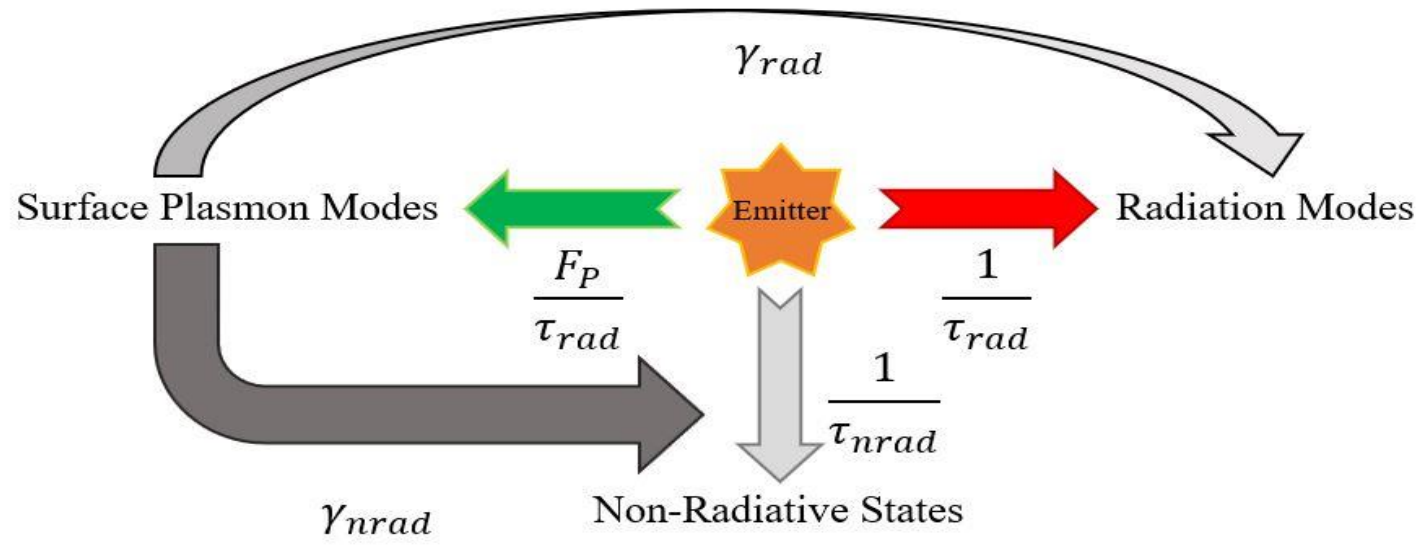

Figure 2.13 An illustration of the two-step emission process and coupling rates: 1) energy transfer from the emitter into SP modes, and 2) the radiative coupling of those SP modes into radiation modes (adapted from [75]).

In general, the more tightly confined the modes with larger Purcell enhancements have a relatively large non-radiative loss and, based on this tradeoff, the greatest overall enhancement can be realized through the optimization of nanostructure parameters [75]. If the emission frequency is assumed to be close to the SPR of the metal NS:

$\varepsilon_{M}\left(\omega_{0}\right) \approx-2 \varepsilon_{D}$

The effective volume $\left(V_{\text {eff }}\right)$ of the eigenmode via the total energy can be calculated:

$U=\int_{r<a} \frac{1}{2} \varepsilon_{0} \frac{\partial\left(\omega \varepsilon_{M}\right)}{\partial \omega} E^{2} d^{3} r+\int_{r>a} \frac{1}{2} \varepsilon_{0} \varepsilon_{D} E^{2} d^{3} r=\frac{1}{2} \varepsilon_{0} \varepsilon_{D} E_{\max }^{2} V_{e f f}$ 
Solving for $V_{\text {eff: }}$

$V_{e f f}=\frac{4}{3} \pi a^{3}\left(1+\frac{1}{2 \varepsilon_{D}}\right)$

A noteworthy observation is the effective volume found in (87) is very close to the volume of a sphere [75]. Considering a dipole oriented normal to the surface in the $z$-direction and positioned a distance $(d)$ from the particle surface, the effective SP mode densities are:

$\rho_{S P}=\frac{L(\omega)}{V_{e f f}}\left(\frac{a}{a+d}\right)^{6}$

The normalized Lorentzian line shape of the dipole oscillation is:

$L(\omega)=\frac{\gamma_{d} / 2 \pi}{\left(\omega-\omega_{0}\right)^{2}+\gamma_{d}^{2} / 4}$

The SP frequency contained above in (89) is given in (82). As shown in equation (48), the decay rate of the dipole combines the radiative and non-radiative components. The non-radiative decay rate can be estimated as the SP mode energy decay due to metal absorption:

$\left(\frac{d U}{d t}\right)_{n r a d}=\int_{r<a} \frac{1}{2} \varepsilon_{0} \operatorname{Im}\left[\frac{\partial\left(\omega \varepsilon_{M}\right)}{\partial \omega}\right] \omega E^{2} d^{3} r=\left(\frac{4}{3} \pi a^{3}\right)\left\{\frac{1}{2} \varepsilon_{0} \operatorname{Im}\left[\frac{\partial\left(\omega \varepsilon_{M}\right)}{\partial \omega}\right] \omega E^{2}\right\}$

$\left(\frac{d U}{d t}\right)_{n r a d}=-\frac{\gamma}{2} U$

$\therefore \gamma_{\text {nrad }}=\frac{\gamma}{2}$ 
One can infer from (92) that essentially half of the total energy is contained within the metal [75]. Using the standard dipole radiation formula, the radiative decay rate becomes:

$$
p=4 \pi \varepsilon_{0} \frac{\varepsilon_{M}-\varepsilon_{D}}{3 \varepsilon_{D}} a^{3}\left(\frac{3 \varepsilon_{D}}{\varepsilon_{M}+2 \varepsilon_{D}} E_{0}\right) \approx-2 \pi \varepsilon_{0} a^{3} E_{\max }
$$

The radiating power of the dipole at the SPR frequency is:

$$
P_{r a d}=-\left(\frac{d U}{d t}\right)_{r a d}=\frac{\omega_{0}^{4} \eta^{3}}{12 \pi \varepsilon_{0} c^{3}} p^{2}=\left(\frac{2 \pi a}{\lambda_{d}}\right)^{3} \frac{\omega_{0}}{1+2 \varepsilon_{D}} U
$$

Above, $c$ is the free-space speed of light, $\eta$ is the refractive index of the dielectric, and $\lambda_{D}=\lambda / \eta$ is the wavelength of emission in the dielectric. (94) holds true only for small NP sizes relative to the wavelength [75]. This leads to the following simplification:

$\gamma_{r a d}=\left(\frac{2 \pi a}{\lambda_{d}}\right)^{3} \frac{\omega_{0}}{1+2 \varepsilon_{D}}$

which then results in the radiative coupling efficiency of the SP mode:

$\eta_{p r}=\frac{\gamma_{r a d}}{\gamma_{r a d}+\gamma_{n r a d}}=\frac{Q \chi^{3}}{1+Q \chi^{3}}$

$\chi$ is the normalized sphere radius $\left(2 \pi a / \lambda_{D}\right)$ and $Q$ is the effective quality factor:

$$
Q=\frac{2 \omega_{0}}{\left(1+2 \varepsilon_{D}\right) \gamma}
$$

For an $\mathrm{Au} / \mathrm{GaN}$ system, $Q=0.38$ at an SPR frequency ( $\hbar \omega)$ equal to $1.95 \mathrm{eV}$ [75]. Radiative coupling efficiency increases as NP volume increases and is due to the increase in the number of free electrons present in the particle (i.e., an increased collective effect of the electrons in the metal emitting in phase) [75]. 
Assuming a $z$-polarized SP dipole, the mode densities of the radiation continuum becomes:

$\rho_{\text {rad }}=\frac{1}{3 \pi^{2}}\left(\frac{2 \pi}{\lambda_{D}}\right) \frac{1}{\omega_{0}}$

The Purcell factor at resonance can thus be represented as:

$F_{P}\left(\omega_{0}\right)=\frac{\rho_{S P}}{\rho_{\text {rad }}}=\left[V_{e f f}^{-1} L\left(\omega_{0}\right)\left(\frac{a}{a+d}\right)^{6}\right]\left[\frac{1}{3 \pi^{2}}\left(\frac{2 \pi}{\lambda_{D}}\right)^{3} \frac{1}{\omega_{0}}\right]^{-1}=\frac{9 \varepsilon_{D} Q}{\chi^{3}\left(1+Q \chi^{3}\right)}\left(\frac{\chi}{\chi+\chi_{d}}\right)^{6}$

where $\chi_{d}=2 \pi d / \lambda_{D}$. The Purcell factor above exhibits a strong dependence on the size of the metal NP and diminishes rapidly as the separation distance $(d)$ between the NP and the emitter increases [75]. In conjunction, the Purcell factor undergoes an initial increase with the radius of the sphere (adhering to the factor $\left(\chi \mid \chi+\chi_{d}\right)^{6}$ contained in (99)), but then decreases with an increase in sphere volume $\left(\chi^{3}\right)$ [75]. For emitters close to the sphere's surface $\left(\chi_{d}<<\chi\right)$, the Purcell factor decreases with sphere volume. This aforementioned trend between the Purcell factor and sphere volume is fundamentally opposite of that of the coupling efficiency laid out in (96). If the radiative decay rate of the SP mode contained in the Lorentzian shape denominator in (89) is set to $\gamma_{d}=\gamma_{\text {nrad }}=\gamma / 2$ (as in (92)), these opposing trends cancel [75]. This negation effectively means that the overall enhancement efficiency is independent of the size of the NP with the answer derivable from a quasi-static model [75]. Consideration of radiative decay revises the overall picture of enhancement resulting in a strong size-enhancement factor dependence. Thus, the enhancement factor calculation must be transformed to the ratio of luminescence efficiency while in the presence of SP modes:

$\eta_{S P}=\frac{\tau_{r a d}^{-1}+\tau_{r a d}^{-1} F_{p} \eta_{p r}}{\tau_{n r a d}^{-1}+\tau_{r a d}^{-1}\left(F_{p}+1\right)}$

The original radiative efficiency:

$\eta_{\text {rad }}=\frac{\tau_{\text {rad }}^{-1}}{\tau_{\text {rad }}^{-1}+\tau_{\text {rad }}^{-1}}$ 
Therefore, the enhancement factor arising due to a single metal NP:

$F_{S P}=\frac{\eta_{S P}}{\eta_{p r}}=\frac{1+F_{P} \eta_{p r}}{1+F_{P} \eta_{\text {rad }}}$

Equation (102) suggests that, for any enhancement via a single metal NP to occur (i.e., $F_{\text {single }}>1$ ), the SP mode's radiative coupling efficiency must be of greater magnitude than the original radiative efficiency of the quantum emitter [75]. Considering quantum emitters of low intrinsic radiative efficiency (i.e., $F_{p} \eta_{r a d}<<1$ ):

$F_{\text {single }} \approx 1+F_{P} \eta_{p r}=1+\frac{9 \varepsilon_{D} Q^{2}}{\left(1+Q \chi^{3}\right)^{2}}\left(\frac{\chi}{\chi+\chi_{d}}\right)^{6}$

In the case of small NPs $\left(Q \chi^{3}\right)$ where the non-radiative decay rate of the SP modes dominates, the result is virtually identical to the quasi-static approximation where the enhancement factor is weakly dependent upon NP size [74-75]. However, when the emitter is in close proximity to the NP surface (i.e., $\chi_{d} \approx 0$ ), the resulting enhancement shows close to zero dependence on NP size [75]. Interpretation of this observation leads one to conclude that, for an emitter with a small original radiative efficiency $\left(\eta_{\text {rad }}<<1\right)$, the foremost concern is the energy transfer from the emitter into SP modes prior to any non-radiative processes [74-75, 174]. This process overwhelmingly favors small NP sizes for a small effective SP mode volume, but small effective mode volumes result in small radiative coupling efficiency [75]. Consequently, the enhancement factor increase due to a reduction in NP volume rapidly saturates for emitters with small intrinsic radiative efficiencies, but changes when examining emitters with nonzero radiative decay rates - a strong dependence on NP size [75]. The enhancement factor therefore exhibits a strong dependence on NP dimensions with peak enhancement occurring where particle radius is still small enough to furnish smaller $V_{\text {eff }}$ and an enhanced Purcell factor, yet sufficiently large enough to ensure strong radiative coupling of the SP mode [75]. For a higher original radiative efficiency, energy transfer (like FRET) from the emitter to SP modes becomes less of a concern while the concern of how efficient energy transfer from the SP modes into free-space radiation modes grows [75]. 
Table 2.7 presents experimental fluorescence enhancement factors of certain fluorescent labels and their intrinsic quantum yields (where available) used in conjunction with chemically synthesized metallic NPs and the type of SP modes supported by these nanostructures, respectively. Table 2.8 lists the same, but for NPs fabricated using lithography.

Table 2.7 Experimental fluorescence enhancement factors (EF) of certain fluorescent labels used in conjunction with chemically synthesized metallic NPs, the intrinsic quantum yields of the fluorophores (where available), and the type of SP modes supported by these nanostructures,

\begin{tabular}{|c|c|c|c|c|}
\hline Chemically Synthesized Single NPs (SP Mode) & EF & Fluorophore $\left(\eta_{0}\right)$ & $f$ & Reference \\
\hline Gold Core Dielectric Shell (LSP) & 40 & IR800 (0.07) & & [142] \\
\hline Silver NS (LSP) & $13-15$ & AF488 (0.92) & & [111] \\
\hline Gold NS (LSP) & $8-10$ & Nile Blue $(0.8)$ & & [149] \\
\hline Aggregated Silver NSs (LSP) & 170 & Atto $655(0.13)$ & & {$[150]$} \\
\hline Silver NS on Silver Film (SPP coupled with LSP) & 1,000 & Perylene Diimide & High & [151] \\
\hline Gold Nanorod (LSP) & 20.8 & Oxazine-725 & & {$[12]$} \\
\hline Hollow Silver NS (LSP) & 300 & Cy5 (0.28) & & {$[152]$} \\
\hline Silver Core Dielectric Shell (LSP) & 94 & Octadecyl Rhodamine B (R18) & & [153] \\
\hline
\end{tabular}

Table 2.8 Experimental fluorescence enhancement factors (EF) of certain fluorescent labels used in conjunction with metallic NPs fabricated using lithography, the intrinsic quantum yields of the fluorophores (where available), and the type of SP modes supported by these

\begin{tabular}{|c|c|c|c|}
\hline Lithography Fabricated Single NPs & $\mathbf{E F}$ & Fluorophore $\left(\eta_{0}\right)$ & Reference \\
\hline Bow-Tie NP (LSP) & 1,340 & TPQDI (0.025) & {$[134]$} \\
\hline Gold Gap Antenna (LSP) & 1,100 & Alexa Fluor 647 with Quencher (0.08) & {$[136]$} \\
\hline Silver Nanodisks (LSP) & 15 & Сy3 (0.04) & {$[154]$} \\
\hline Gold Nanodisks (LSP) & 15 & CdSe/ZnS QDs (0.3-0.5) & {$[66]$} \\
\hline Gold Nanoholes (Hybrid LSP and SPP) & 82 & Oxazine-720 (0.6) & [4] \\
\hline Silver Nanoholes (Hybrid LSP and SPP) & 110 & Cy5 (0.28) & {$[145]$} \\
\hline Gold Nanodisks on Metal Film (LSP) & 600 & IR800 (0.07) & [155] \\
\hline Gold Nanotriangle (LSP) & 83 & Alexa Fluor $790(0.04)$ & {$[138]$} \\
\hline
\end{tabular}

NP arrays must then be considered for applications involving both efficient energy transfer with specific power and/or wavelength requirements (e.g., larger area devices like LEDs, PhCs, etc.) $[4,14,45,48,50,66,69,75,83-84,86,97,113,130,137,157,163,186]$. 


\subsubsection{Arrays of Metallic Nanoparticles}

Periodic 2D metal NP arrays provide a mechanism for field intensity enhancement through long- and short-distance LSP-coupling [42]. For distances between the NPs similar to the incident wavelength, diffraction (i.e., long-distance) interactions dominate with a narrower LSPR absorption band manifesting itself [43, 61, 68, 87, 115, 131, 137, 172-173]. Conversely, for shorter distances comparable to the LSP decay length $\left(L_{p}\right)$, near-field LSP interactions grow and exhibit an associated LSPR wavelength shift and altered NP-enhanced field intensity profile [42]. Moderate enhancement can be observed for near-field (>10 nm) interactions between plasmoncapable NPs. Diffractive coupling between metal NPs within the array are capable of achieving larger LSPR field enhancement [41-42, 61]. Collective LSPs (cLSPs) arise from this interaction and are particularly strong for symmetrical NP geometries where the refractive index above is equivalent to the refractive index below the array (i.e., phase matching of LSPs at wavelengths coinciding with the LSPR band(s) of the individual NPs comprising the array) [42]. Compared to the LSPs of single NPs, cLSPs trap light at the surfaces of the NPs more efficiently and show strong field enhancement due to the decreased radiative damping [42]. Pictured in Figure 2.14 is the angular dependence as expressed in (46) of SP-coupled emission from SPPs and LRSPPs of an array of gold nanodisks in the reverse-Kretschmann configuration.

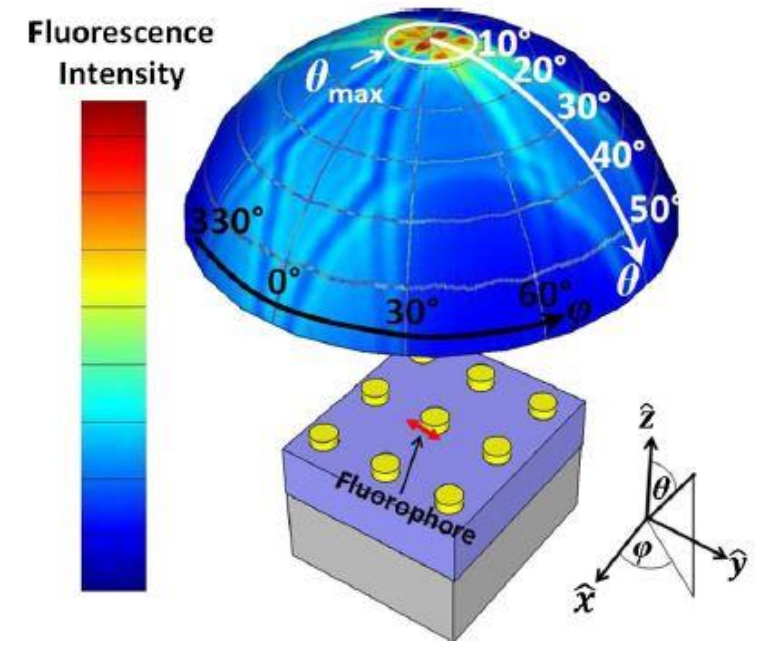

Figure 2.14 Angular distribution of SP-coupled emission resulting from SPPs and LRSPPs observed in the simulation of a reverse-Kretschmann system architecture comprised of an LSPR-supporting array of metal NPs coupled with a dipole point-source showing polar $(\theta)$ and azimuthal $(\phi)$ angle directionality $(f)[42,128-132,139-140,159]$. 
As stated in Section 2.1.3.1 and Section 2.1.3.2, physical models governing light absorption and scattering are only applicable to spherical (Mie) and prolate spheroid (Gans) NPs. A 2D array of NSs separated by distance $R$ atop an active QW emitter (Figure 2.15) similar to the case presented in Figure 2.12 of Section 2.1.6.2 will now be explored.

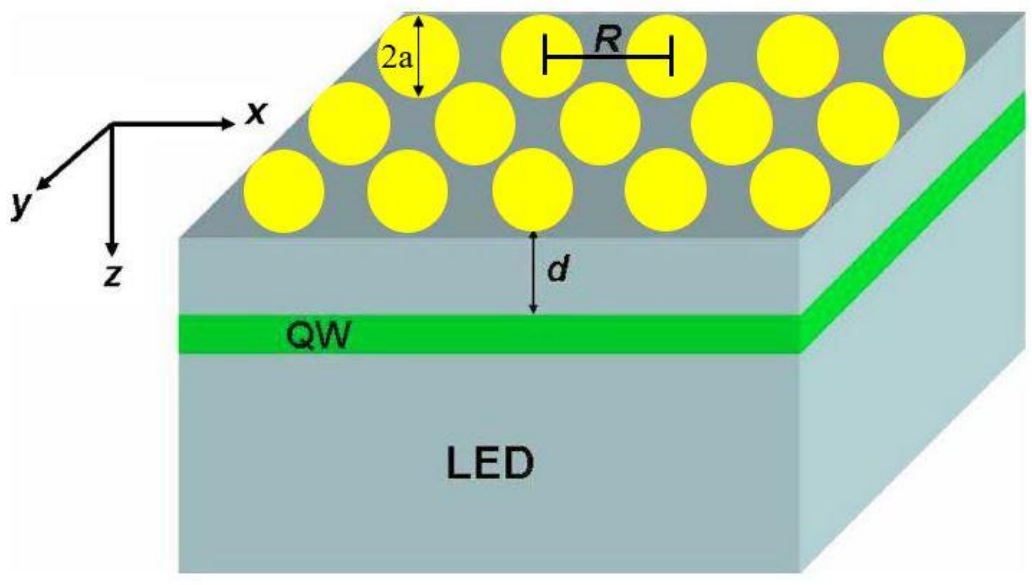

Figure 2.15 2D array of metal NPs atop the active QW region of a LED (adapted from [75]).

SP modes will propagate in $x$ and $y$ as a result of the coupling between the dipoles of neighboring metal NPs characterized by the relationship between frequency and the 2D SP mode wave vectors (i.e., 2D dispersion) [75]. Accounting for the interactions between only the closest neighboring NPs, this dispersion can be mathematically obtained considering the motion for the $m^{\text {th }}, n^{\text {th }}$ dipole $\left(p_{m, n}\right)$ :

$\frac{\partial^{2} p_{m, n}}{d t^{2}}=-\omega_{0}^{2} p_{m, n}-\omega_{0}^{2} p_{m-1, n} \frac{E_{\text {out }}(R, \theta)}{E_{\text {in }}}-\omega_{0}^{2} p_{m+1, n} \frac{E_{\text {out }}(R, \theta)}{E_{\text {in }}}-$

$\omega_{0}^{2} p_{m, n-1} \frac{E_{\text {out }}(R, \theta)}{E_{\text {in }}}-\omega_{0}^{2} p_{m, n+1} \frac{E_{\text {out }}(R, \theta)}{E_{\text {in }}}$

$E_{\text {in }}$ and $E_{\text {out }}$ are the dipole field magnitudes inside $(r<a)$ and outside $(r>a)$ of the sphere as exhibited in equations (78) and (79), respectively [75]. 
These fields are related at near resonance (shown in (85)) by:

$E_{\text {out }}(R, \theta)=E_{\text {in }} \frac{a^{3}}{R^{3}}\left[1+3 \cos ^{2}(\theta)\right]$

For TM-polarized dipole sources where $\theta=\pi / 2$ :

$E_{\text {out }}(R, \theta)=E_{\text {in }} \frac{a^{3}}{R^{3}}$

Taking the above case and applying it to the dipole motion equation (104) yields:

$\frac{\partial^{2} p_{m, n}}{d t^{2}}=-\omega_{0}^{2} p_{m, n}-\omega_{0}^{2} \frac{a^{3}}{R^{3}}\left(p_{m-1, n}+p_{m+1, n}+p_{m, n-1}+p_{m, n+1}\right)$

Substituting:

$p_{m, n}=p_{0} e^{i\left(q_{x} m R+q_{y} n R-\omega_{q} t\right)}$

into equation (107) produces the dispersion relationship in the following tight-band approximation:

$\omega_{q}^{2}=\omega_{0}^{2}\left(1+2 \frac{a^{3}}{R^{3}}\left[\cos \left(q_{x}, R\right)+\cos \left(q_{y}, R\right)\right]\right)$

Presented in (109) is the broad SP band in which each mode is characterized by a wave vector:

$q=q_{x} \hat{x}+q_{y} \hat{y}$ 
The wave vector (i.e., the SP mode dispersion relationship) above can be visually interpreted below in Figure 2.16.

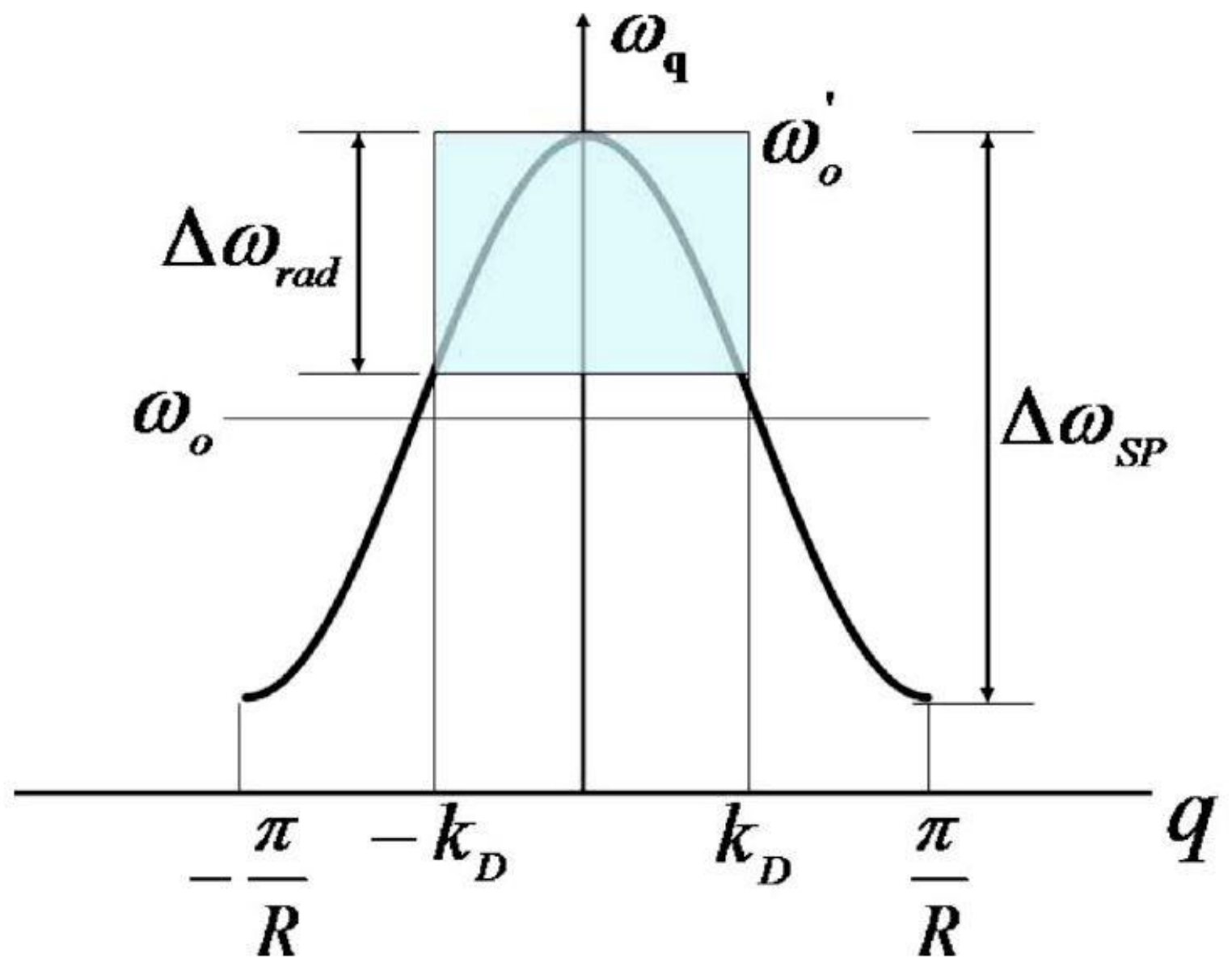

Figure 2.16 2D array SP mode dispersion relationship [75].

The corresponding luminescence then becomes efficiently coupled into the multiple modes inside the broad SP band:

$\Delta \omega_{S P} \approx 4\left(\frac{a}{R}\right)^{3} \omega_{0}$

As an aside, only modes with a small wave vector $\left(|q|<k_{D}=2 \pi / \lambda_{D}\right)$ within the narrower band are capable of coupling into free-propagating dielectric modes [75]. 
These free-propagating dielectric modes are confined within a narrow bandwidth of frequencies near the top of the band (as shown in Figure 2.16):

$\omega_{0}^{\prime}=\left(1+4 \frac{a^{3}}{R^{3}}\right)^{1 / 2} \omega_{0}$

It can be seen that these radiative modes only account for a small fraction of all of the SP modes:

$g_{\text {rad }}=\frac{\pi k_{D}}{(2 \pi / R)^{2}}=\frac{\pi R^{2}}{\lambda_{D}^{2}}$

The quantity above equals unity for sparsely spaced metal arrays where $R>\lambda_{D} / \sqrt{\pi}$ and, since each radiative SP mode contained in the radiative band $\left(\Delta \omega_{\mathrm{rad}}\right)$ is a collective oscillation of $g_{\text {rad }}^{-1} \mathrm{SP}$ dipoles nearly in phase, the radiative decay time should be then be reduced by roughly the same factor $g_{\text {rad }}$ [75]. The radiative out-coupling efficiency can be modified to:

$\eta_{p r, a r r a y}=\frac{\gamma_{r a d} g_{r a d}^{-1}}{\gamma_{r a d} g_{r a d}^{-1}+\frac{\gamma}{2}}=\frac{Q \chi^{3}}{g_{r a d}+Q \chi^{3}}$

For densely packed NSs with small radiative SP modes $\left(g_{\text {rad }}<<1\right)$, the coupling efficiency approaches unity [75]. These broadening radiative modes then become:

$\gamma_{d, \text { array }}=\left(\frac{\lambda_{D}^{2}}{\pi R^{2}}\right) \gamma_{r a d}+\frac{\gamma}{2}$

The normalized line shape undergoes transformation to:

$L_{r a d}(\omega)=\frac{\gamma_{d, a r r a y} / 2 \pi}{\left(\omega-\omega_{0}^{\prime}\right)^{2}+\gamma_{d, \text { array }}^{2} / 4}$ 
The resulting combined Purcell factor:

$F_{P, r a d}=g_{r a d} \frac{9 \pi \varepsilon_{D} \gamma Q}{4 \chi^{3}} \bar{L}_{r a d}\left(\omega_{0}^{\prime}\right) \frac{a^{6}}{\left[(a+d)^{2}+\frac{R^{2}}{6}\right]^{3}}$

Accounting for the average separation between the emitters and the 2D metal NSs:

$\bar{d}^{2}=(a+d)^{2}+\frac{R^{2}}{6}$

Taking the average of the normalized Lorentzian line shape within $\Delta \omega_{\text {rad }}$ results in:

$\bar{L}_{\text {rad }}\left(\omega_{0}^{\prime}\right)=\frac{\int_{0}^{k} d L_{r a d}(2 \pi q) d q}{\int_{0}^{k} d(2 \pi q) d q}$

The non-radiative modes remaining of a larger wave vector $\left(|q|>k_{D}=2 \pi / \lambda_{D}\right)$ within $\Delta \omega_{S P}$ have a broadening of $\gamma / 2$ with a normalized Lorentzian line shape:

$L_{n r a d}(\omega)=\frac{\gamma / 2 \pi}{\left(\omega-\omega_{0}^{\prime}\right)^{2}+\gamma^{2} / 4}$

The combined Purcell factor of the electroluminescent coupling into these modes is:

$F_{P, n r a d}=\left(1-g_{r a d}\right) \frac{9 \pi \varepsilon_{D} \gamma Q}{4 \chi^{3}} \bar{L}_{n r a d}\left(\omega_{0}^{\prime}\right) \frac{a^{6}}{\left[(a+d)^{2}+\frac{R^{2}}{6}\right]^{3}}$

No emission at all will occur in the case put forth in equation (121) [75]. Describing the nonradiative band $\left(\Delta \omega_{\text {nrad }}\right)$ :

$\Delta \omega_{\text {nrad }}=\Delta \omega_{S P}-\Delta \omega_{\text {rad }}$ 
The average of the normalized Lorentzian line shape $\left(\bar{L}_{\text {nrad }}\left(\omega_{0}^{\prime}\right)\right)$ within $\Delta \omega_{\text {nrad }}$ can be numerically derived similar to (116) only taking the average within the first Brillouin zone where $|q|>k_{D}$ [75]. The Purcell factor obtained from a 2D array of metal NSs is at least one order of magnitude less than that of a single NS having equal separation between the emitter and the metal NS [75]. This disparity in Purcell factors has two origins: 1) the fact that energy transfer from the emitter into the SP modes of the 2D NS array can no longer be resonant since the SP modes of the array spread into $\Delta \omega_{S P}$, and 2) the average separation between the emitter and metal NSs is effectively larger [75]. Finally, the fluorescence enhancement factor of a 2D array of metal NSs reads:

$F_{\text {array }}=\frac{1+F_{P, \text { rad }} \eta_{\text {pr,array }}}{1+\left(F_{P, \text { rad }}+F_{P, \text { nrad }}\right) \eta_{\text {rad }}}$

Compared to the enhancement factor due to a single metal NS in equation (102), the enhancement granted from a 2D array of metal NSs is significantly reduced by fluorescent coupling to the non-radiative modes within $\Delta \omega_{S P}$ via the Purcell factor $F_{P, \text { nrad }}$ [72-75]. The enhancement of the 2D metal NS array depends on both the radii of the spheres and the spacing between them [75]. In summary, a 2D array of smaller NSs provide better fluorescent enhancement for inefficient, lower quantum yield emitters, while an array of larger 2D NSs can be utilized to improve the enhancement of emitters that are intrinsically more efficient. This trend is explained by the fact that $F_{P}$ is larger for smaller metal NPs while $\eta_{p r, a r r a y}$ is larger for larger metal NPs. In cases of smaller $\eta_{r a d}, F_{P}$ is of greater importance while $\eta_{\text {prarray }}$ tends to dominate for higher $\eta_{\text {rad }}$ [75].

\subsection{Fluorescent Molecules}

\subsubsection{Fluorophores}

Fluorescent molecules, fluorophores, or fluor are particles that respond distinctly to light compared to other molecules [127, 183]. An incident excitation photo is absorbed by an electron of the fluorophore causing the fluorophore to go from equilibrium (i.e., ground) to a higher energy excited state [114]. During this excitation, some energy is dissipated by intramolecular collisions, or transferred to a molecule in proximity to the fluorophore (i.e., quenching) $[15,18,72,127,183]$. 


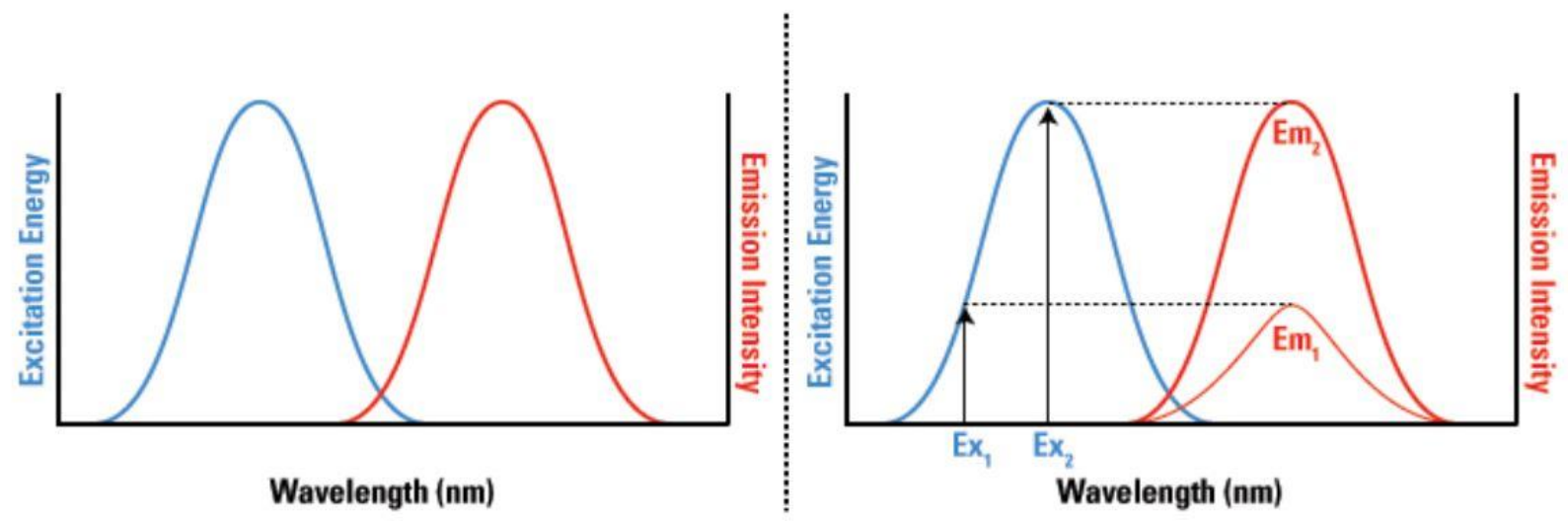

Figure 2.17 Excitation and emission spectra of a fluorophore including the correlation between the amplitude of photonic excitation and the intensity of photonic emission [183].

Presented above in Figure 2.17 is the spectra for both mono- and polyatomic fluorophores indicating the wavelengths corresponding to the excitation and emission minimum and maximum signal intensities [183]. An important point to note is that, while the emission wavelength is independent of the excitation due to the partial energy loss prior to emission, the intensity of the emission is directly proportional to the amplitude of the excitation wavelength $\left(\mathrm{Ex}_{1}\right.$ and $\mathrm{Ex}_{2}$ and the respective emission intensities they correspond to $-\mathrm{Em}_{1}$ and $\mathrm{Em}_{2}$ ) [183]. Fluorophore brightness is a quantity determined by two factors: 1) molar extinction coefficient $(\varepsilon)$ and quantum yield. $\varepsilon$ is defined as the amount of light that a fluorophore is capable of absorbing at a given wavelength $\left(\mathrm{M}^{-1} \mathrm{~cm}^{-1}\right)$ while the quantum yield is the ratio of photons emitted by the fluorophore to the number of photons absorbed (equation (56)) [114, 127, 183, 185].

The energy remaining causes photon emission (i.e., fluorescence) as the fluorophore's electron settles down to its ground state. The emitted photon resulting from the ground-excitationground process usually carries less energy than the excitation photon, resulting in a longer, redshifted wavelength distinguishable from the wavelength of excitation $[127,183]$. The fluorescence process is cyclical meaning that, unless the fluorophore itself becomes irreversibly damaged (i. e., photobleached), it can be excited repeatedly [183]. As long as this case holds true, the fluorophore is capable of emitting numerous photons. Photobleaching is the irreversible damage sustained by a fluorophore due to either prolonged exposure to the excitation source or to high-intensity light [183]. The experimental catastrophe of photobleaching can be minimized or avoided entirely by exposing the fluorophore(s) to the lowest possible excitation intensity for the shortest period of time still allowing for signal detection. 
Optimal detection methods to combat photobleaching include the use of high sensitivity CCD cameras, high numerical aperture objective, and/or the wide bandpass emission filters [183]. Other approaches include using fluorophores of higher photostability and/or using antifade reagents to protect the fluor(s) against photo bleaching [183]. Similar to QDs, fluorophores possess excitation and emission spectra unique to the label itself $[114,127,183,185]$. These wavelengths are discrete for monoatomic fluorophores; however, for polyatomic fluorophores, these spectra are much broader [183].

Quantum yield, as previously mentioned in Section 2.1.4, describes the optical efficiency of the fluorophore with a maximum obtainable value of unity $[8,62,127,183]$. Fluorophores have seen extensive use in biological research over the past century undergoing significant development courtesy of advancements in fluorescence chemistry and other technical discoveries [183]. The seemingly boundless selection of fluorophores available today provides tremendous flexibility, performance, and variation across a plethora of research and medical applications [127, 174, 183]. Selection criteria for a fluorophore include its initial brightness, photostability in buffer, stain index, laser line, common filter set, excitation and emission maxima, and performance in superresolution microscopy (SRM) [183]. Buffer photostability is a relative measurement of the percentage of initial fluorescence remaining after 30 seconds of continuous illumination using a 40x/1.4NA objective with a $100 \mathrm{~W}$ mercury $(\mathrm{Hg})$ arc lamp light source using a phosphate buffer to mount the samples. Stain index is a normalized value derived by subtracting the mean fluorescence intensity (MFI) of the negative population from the MFI of the positive population and then dividing that result by twice the standard deviation of the negative population; this allows the user to compare the brightness of various fluorescent dyes in cytometry applications. Laser line denotes the commonly used laser line(s) for cytometry flow detection. Common filter set represents the standard microscope filter set that produces the best or ideal imaging results. The excitation and emission maxima are the excitation and emission peaks of the fluorophore. Finally, SRM performance is a normalized value that allow for the comparison of various fluorophore brightness in SRM applications determined within the optimum buffer type for each fluorophore, respectively [183]. Fluorophores can be categorized into three main groups: 1) organic dye molecules, 2) biological fluorophores, and 3) QDs. 
Fluorescent compounds first saw use in biological research through the introduction of the synthetic organic dye molecule fluorescein [127, 174, 183]. Multiple derivatives of fluorescein have been produced to improve the photostability and solubility in bioconjugation techniques, namely fluorescein isothiocyanate (FITC), rhodamine (tetramethyl rhodamine isothiocyanate (TRITC)) and other commercial variants [183]. Advantages inherent to organic dye molecules compared to other biological fluorophores include their visible wavelength excitation and emission spectra, macromolecule (i.e., antibodies, Avidin, and biotin) crosslinking biocompatibility, optimal quantum yield, and smaller size [183].

The first biological fluorophore employed in research applications occurred in the 1990s with the cloning of the green fluorescent protein (GFP) from the naturally bioluminescent jelly fish Aequorea victoria [183]. This clone fluorophore was initially a gene expression reporter later used to derive other proteins including phycobiliproteins (allophycocyanin), phycocyanin, phycoerythrin, phycoerythrocyanin, among many others designed specifically for biological expression strategies. A glaring advantage of biological fluorophores and fluorescent proteins lies in the use of expression plasmids. Expression plasmids can be introduced into either bacteria, cells, organs or whole organisms to drive the expression of the fluorophore either alone or fused to a protein of interest depending on application [183]. However, the use of fluorescent proteins can lead to the expression of large amounts of light-producing, reactive oxygen $\left(\mathrm{O}_{2}\right)$ species producing proteins that introduce spurious artifacts or toxicity and can be quite time consuming [183]. Additionally, the larger size of the fluorescent protein can modify the normal biological function of the fluorophore-conjugated cellular protein(s) and lacks the level of photostability, and sensitivity offered by synthetic fluorescent dyes [183]. General fluorescence detection involves the use of an excitation source (i.e., a lamp (xenon (Xe) arc and mercury $(\mathrm{Hg}$ ) vapor) laser, or photodiode), a selected fluorophore, a filter for the isolation of specific wavelengths used in the excitation of the fluorophore, and a detector used for recording the electronic output of the fluorescence signal [183]. Common fluorescence instrumentation includes the following configurations: 1) a fluorescent microscope that detects localized fluorophores both two and three dimensional samples, 2) a fluorescence scanner like a microarray reader that detects fluorophores in a two dimensional sample space, 3) a spectrofluorometer and microplate reader used to record the average fluorescence intensity in a sample, and 4) flow cytometers used to analyze the fluorescence of individual cells in a sample [183]. 
Advancements in fluorescence signal quantification allow for the measurement of a broad range of parameters of interest including the amount of DNA, RNA, or protein present in a sample, the amount of fluorophore localized cells or discrete cellular compartments, cell number, specific DNA, RNA or protein sequences, enzyme activity, rate of cell movement in intracellular components, rate of gene expression in protein synthesis, and viability [183]. Specialized software is required for quantization of the fluorescence signal and, depending on experimental approach, fluorescent standards may be required to calibrate the instrumentation used [183]. Additionally, covalent attachment (i.e., conjugation) of a fluorophore to a functional group present in another molecule (antibody, nucleic acid, protein, etc.) is necessary for successful labeling in order for the fluorescence process to occur [174, 183]. Tables 2.9-2.10 summarize some commercially available fluorophores/fluorescent compounds including their excitation and emission maxima, quantum yields, excited state lifetimes (where available), molar mass (MM) (where available), and extinction coefficients $(\varepsilon)$ (where available).

Table 2.9 Other commonly used fluorescent labels showing label name, excitation and emission peak wavelengths, and reported quantum yield

\begin{tabular}{|c|c|c|c|}
\hline Fluorescent Label & Excitation Peak $(\lambda)$ & Emission Peak $(\lambda)$ & Quantum Yield \\
\hline Acridine Orange & $493 \mathrm{~nm}$ & $535 \mathrm{~nm}$ & 0.46 \\
\hline Benzene & $248 \mathrm{~nm}$ & $300 \mathrm{~nm}-350 \mathrm{~nm}$ & 0.04 \\
\hline Chlorophyll-A & $440 \mathrm{~nm}$ & $685 \mathrm{~nm}$ & 0.23 \\
\hline CFP & $433 \mathrm{~nm}$ & $475 \mathrm{~nm}$ & 0.4 \\
\hline Cy3 & $554 \mathrm{~nm}$ & $568 \mathrm{~nm}$ & 0.14 \\
\hline Cy5 & $652 \mathrm{~nm}$ & $672 \mathrm{~nm}$ & 0.58 \\
\hline DAPI & $350 \mathrm{~nm}$ & $470 \mathrm{~nm}$ & 0.16 \\
\hline Eosin & $521 \mathrm{~nm}$ & $544 \mathrm{~nm}$ & 0.92 \\
\hline Fluorescein & $437 \mathrm{~nm}$ & $515 \mathrm{~nm}$ & 0.6 \\
\hline GFP & $488 \mathrm{~nm}$ & $507 \mathrm{~nm}$ & 0.22 \\
\hline mCherry & $587 \mathrm{~nm}$ & $610 \mathrm{~nm}$ & 0.97 \\
\hline Rhodamine-B & $555 \mathrm{~nm}$ & $627 \mathrm{~nm}$ & 0.84 \\
\hline R-Phycoerythrin & $565 \mathrm{~nm}$ & $573 \mathrm{~nm}$ & 0.76 \\
\hline YFP & $516 \mathrm{~nm}$ & $529 \mathrm{~nm}$ & \\
\hline
\end{tabular}


Table 2.10 Alexa fluorophores (fluor) available from ThermoFisher Scientific showing label name, excitation and emission peak wavelengths, reported quantum yield, lifetime of excited state, molar mass (MM), and extinction coefficient $(\varepsilon)$ [182].

\begin{tabular}{|c|c|c|c|c|c|c|}
\hline Fluorophore & Excitation Peak $(\lambda)$ & Emission Peak $(\lambda)$ & $\begin{array}{c}\text { Quantum } \\
\text { Yield }\end{array}$ & $\begin{array}{c}\text { Lifetime } \\
\text { (ns) }\end{array}$ & $\begin{array}{c}\text { MM } \\
\text { (g/mol) }\end{array}$ & $\varepsilon\left(\mathbf{M}^{-1} \mathrm{~cm}^{-1}\right)$ \\
\hline Alexa Fluor 350 & $346 \mathrm{~nm}$ & $442 \mathrm{~nm}$ & - & - & 410 & 19,000 \\
\hline Alexa Fluor 405 & $401 \mathrm{~nm}$ & $421 \mathrm{~nm}$ & - & - & 1,028 & 35,000 \\
\hline Alexa Fluor 488 & $490 \mathrm{~nm}$ & $525 \mathrm{~nm}$ & 0.92 & 4.1 & 643 & 73,000 \\
\hline Alexa Fluor 532 & $532 \mathrm{~nm}$ & $554 \mathrm{~nm}$ & 0.61 & 2.5 & 721 & 81,000 \\
\hline Alexa Fluor 546 & $556 \mathrm{~nm}$ & $573 \mathrm{~nm}$ & 0.79 & 4.1 & 1,079 & 112,000 \\
\hline Alexa Fluor 555 & $555 \mathrm{~nm}$ & $580 \mathrm{~nm}$ & 0.10 & 0.3 & $\sim 1,250$ & 155,000 \\
\hline Alexa Fluor 568 & $578 \mathrm{~nm}$ & $603 \mathrm{~nm}$ & 0.69 & 3.6 & 792 & 88,000 \\
\hline Alexa Fluor 594 & $590 \mathrm{~nm}$ & $617 \mathrm{~nm}$ & 0.66 & 3.9 & 820 & 92,000 \\
\hline Alexa Fluor 647 & $650 \mathrm{~nm}$ & $665 \mathrm{~nm}$ & 0.33 & 1.0 & $1,155.06$ & 270,000 \\
\hline Alexa Fluor 680 & $679 \mathrm{~nm}$ & $702 \mathrm{~nm}$ & 0.36 & 1.2 & $\sim 1,150$ & 183,000 \\
\hline Alexa Fluor 750 & $749 \mathrm{~nm}$ & $775 \mathrm{~nm}$ & 0.12 & 0.7 & $\sim 1,300$ & 290,000 \\
\hline
\end{tabular}

\subsubsection{Quantum Dots}

QDs, also known as nanoparticle semiconductors or fluorescent nanocrystals, are a specific type of fluorophore that were theorized in the 1970s and created in the early 1980s [181-182]. When these semiconducting nanoparticles are sufficiently small, quantum effects become important and limit the energies at which electrons and holes can exist within the particles [66, $115-118,146,181]$. The optical properties of QDs can be acutely tuned depending on their composition, morphology, and size allowing for tight control over the spectral characteristics of fluorescence (i.e., their absorbance and emission at specific wavelengths) [66, 115-118, 146, 181]. The aforementioned tunability of QDs render them useful across a broad range of applications such as catalysis, various electronic display and lighting systems, information storage, medical research, and photovoltaics [66, 115-118, 146, 181]. QDs are reliable in fluorescence biosensors because of their atom-like energy states that contribute to special optical properties such as a particle-size dependent fluorescence.

To date, colloidal QDs provide the widest range of applications across bioanalytics and biolabeling [181-182]. Research throughout bioengineering and medical literature has allowed for the observation and study of cell process at the single molecule level which has resulted in improved diagnoses and treatment options for life-threatening diseases like breast cancer, leukemia, prostate cancer, etc. [181-182]. 
The early detection, diagnosis, and subsequent treatment and therapy of cancer is achieved by utilizing QDs as active sensor elements in high-resolution cellular imaging devices whereby the fluorescence properties of the QDs are changed as a result of their interaction with an analyte. Additionally, QDs can be employed in passive biosensor architectures where select receptor molecules are physically conjugated to the QD surface [181-182].

The primary concern facing QD usage in medical applications is their toxicity due to the existence of heavy metals like cadmium $(\mathrm{Cd})$ and lead $(\mathrm{Pb})$ present in their composition; thus, prohibiting their direct injection into the human body as well as introducing potential environmental hazards [181]. To combat the rise of nanomaterial incorporation in biomedical applications, the above issues must be addressed. Ideally, biocompatible QDs will need to be developed if they are to truly revolutionize medical detection and diagnostics [181]. Figure 2.18 shows the overall structure of a QD probe conjugate including structural elements drawn roughly to scale. Table 2.11 contains manufacturer provided specifications of $\mathrm{CdSe} / \mathrm{ZnS}$ core-shell QDs. More commercially available QDs and their specifications can be found in the Appendix.

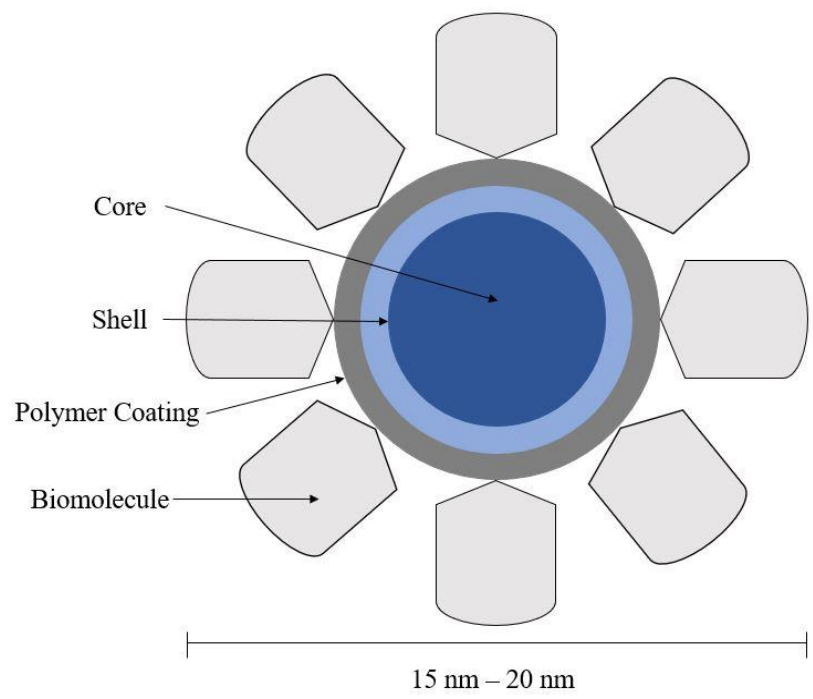

Figure 2.18 Structure of a QD probe conjugated with a biomolecule including structural elements, roughly drawn to scale (adapted from [182]). 
Table 2.11 Cadmium-selenide/zinc-sulfide (CdSe/ZnS) core-shell QDs coated with octadecylamine (ODA) stabilizing ligand in toluene available from NNCrystal, US Corporation showing label product number, absorbance (excitation) and emission peaks, reported quantum yield, and concentration in solution [184].

\begin{tabular}{|c|c|c|c|c|}
\hline Label & Excitation Peak Range $(\boldsymbol{\lambda})$ & Emission Peak Range $(\boldsymbol{\lambda})$ & Quantum Yield & $\begin{array}{c}\text { Concentration } \\
(\mathbf{n m o l} / \mathbf{m g})\end{array}$ \\
\hline CZ500 & $500 \pm 10 \mathrm{~nm}$ & $535 \mathrm{~nm}-550 \mathrm{~nm}$ & $>0.40$ & 2.5 \\
\hline CZ520 & $520 \pm 10 \mathrm{~nm}$ & $530 \mathrm{~nm}-545 \mathrm{~nm}$ & $>0.40$ & 2.0 \\
\hline CZ540 & $540 \pm 10 \mathrm{~nm}$ & $545 \mathrm{~nm}-565 \mathrm{~nm}$ & $>0.40$ & 2.0 \\
\hline CZ560 & $560 \pm 10 \mathrm{~nm}$ & $565 \mathrm{~nm}-580 \mathrm{~nm}$ & $>0.40$ & 1.0 \\
\hline CZ580 & $580 \pm 10 \mathrm{~nm}$ & $585 \mathrm{~nm}-600 \mathrm{~nm}$ & $>0.40$ & 1.0 \\
\hline CZ600 & $600 \pm 10 \mathrm{~nm}$ & $600 \mathrm{~nm}-620 \mathrm{~nm}$ & $>0.40$ & 1.0 \\
\hline CZ620 & $620 \pm 10 \mathrm{~nm}$ & $620 \mathrm{~nm}-640 \mathrm{~nm}$ & $>0.40$ & 1.0 \\
\hline CZ640 & $640 \pm 10 \mathrm{~nm}$ & $640 \mathrm{~nm}-660 \mathrm{~nm}$ & $>0.40$ & 1.0 \\
\hline
\end{tabular}




\subsubsection{History of the FDTD Method}

Table 2.12 Timeline of significant events in the history of FDTD [190]. Continues.

\begin{tabular}{|c|c|}
\hline Year & Event \\
\hline 1966 & Kane S. Yee's initial proposal - the beginning of what would become the FDTD method \\
\hline 1975 & Taflove and Brodwin first apply Yee's method to biological heating and EM scattering \\
\hline 1977 & Holland introduces THREDE - first software program based on Yee's algorithm for transient EMP simulation \\
\hline 1977 & Weiland pioneers the Finite-Integration Technique (FIT) \\
\hline 1980 & Taflove introduces the FDTD acronym \\
\hline 1981 & Holland and Simpson develop a sub-cell model for modeling thin wires \\
\hline 1981 & Mur introduces first second-order absorbing boundary for FDTD \\
\hline 1981 & Gilbert and Holland propose thin-cell sub-cell model \\
\hline $1982-1983$ & $\begin{array}{l}\text { Umashankar and Taflove introduce the TF/SF plane-wave boundary and the near-field to far-field transformation in two and } \\
\text { three dimensions }\end{array}$ \\
\hline 1983 & Holland derives first non-orthogonal FDTD (N-FDTD) method \\
\hline 1986 & Higdon's Absorbing Boundary Operators \\
\hline 1987 & Umashankar, et al., develop sub-cell thin-wire model for FDTD \\
\hline 1988 & Sullivan, Gandhi and Taflove - first full human model via the FDTD method \\
\hline 1989 & Chu and Chadhuri apply FDTD to optical structures \\
\hline 1990 & $\begin{array}{l}\text { Sheen, et al., compute the scattering-parameters of printed circuits using the FDTD } \\
\text { method }\end{array}$ \\
\hline 1990 & $\begin{array}{l}\text { Shankar, et al., and Madsen and Ziolkowski introduce Finite-Volume Time-Domain } \\
\qquad \text { (FVTD) methods }\end{array}$ \\
\hline 1990 & Sano and Shibata are the first to apply FDTD to opto-electronics \\
\hline 1990-1991 & $\begin{array}{l}\text { Luebbers, et al., and Joseph, et al., develop stable and accurate FDTD models for } \\
\text { frequency-dependent linear dispersive media }\end{array}$ \\
\hline 1991 & Maloney, et al., apply the FDTD method to antenna modeling \\
\hline 1992 & $\begin{array}{l}\text { Computer Simulation Technology (CST) markets the first FDTD-based commercial software MAFIA based on FIT form of } \\
\text { FDTD }\end{array}$ \\
\hline 1992 & $\begin{array}{c}\text { Maloney and Smith develop FDTD method to treat frequency dependent surface } \\
\text { impedance boundary conditions and thin-material sheets }\end{array}$ \\
\hline 1992 & Goorjian, et al., apply FDTD to solve wave propagation in non-linear media \\
\hline 1992 & Jurgens, et al., introduce the Conformal Patch FDTD method \\
\hline 1992 & Betz and Mittra introduce an $\mathrm{ABC}$ that absorbs evanescent waves \\
\hline $1992-1993$ & $\begin{array}{l}\text { Sui, et al.s and Toland, et al, first to model non-linear circuit devices within the } \\
\text { FDTD method }\end{array}$ \\
\hline 1993 & Schneider and Hudson apply FDTD to anisotropic media \\
\hline 1993 & Kunz and Luebbers publish the first textbook on FDTD \\
\hline 1994 & Luebbers, Langdon and Penney form Remcom, Inc., and market XFDTD \\
\hline 1994 & Thomas, et al., couple FDTD model with SPICE for high-speed circuit simulation \\
\hline
\end{tabular}


Table 2.13 Continued. Timeline of significant events in the history of FDTD [190].

\begin{tabular}{|c|c|}
\hline Year & Event \\
\hline 1994 & $\begin{array}{l}\text { J.-P. Berenger pioneers the Perfectly Matched Layer (PML) absorbing media for } \\
\text { simulating unbounded regions with the FDTD method }\end{array}$ \\
\hline 1994 & Chew and Weedon derive a stretched-coordinate form of the PML \\
\hline 1995 & FDTD algorithm for massively parallel computers \\
\hline 1995 & $\begin{array}{l}\text { Madsen introduces the discrete surface integral (DSI) method applying an FDTD-like } \\
\text { algorithm to unstructured grids }\end{array}$ \\
\hline 1995 & Taflove publishes textbook on FDTD (2nd edition in 2000, and $3^{\text {rd }}$ in 2005) \\
\hline $1995-1996$ & $\begin{array}{c}\text { Sacks, Kingsland, Lee, and Lee and Gedney develop an anisotropic media form of } \\
\text { the PML }\end{array}$ \\
\hline 1996 & Bourgeois and Smith develop a full three-dimensional model of a ground penetrating radar \\
\hline 1996 & Dunn, et al., apply FDTD to study the scattering of light by biological cells \\
\hline 1997 & Dey-Mittra conformal-FDTD method for PEC boundaries \\
\hline 1997 & $\begin{array}{c}\text { Hagness, et al, apply FDTD in the area of photonics and model an optical } \\
\text { directional coupler }\end{array}$ \\
\hline 1998 & $\begin{array}{c}\text { Roden, et al., develops a stable FDTD analysis of periodic structures with oblique } \\
\text { incidence }\end{array}$ \\
\hline 1998 & $\begin{array}{c}\text { Hagness, et al., apply FDTD method for microwave imaging for breast cancer } \\
\text { detection }\end{array}$ \\
\hline 2000 & $\begin{array}{l}\text { Zheng, Chen and Zhang develop the first unconditionally stable 3D alternating } \\
\text { direction implicit (ADI) FDTD method }\end{array}$ \\
\hline 2000 & $\begin{array}{l}\text { Roden and Gedney develop the Convolutional PML method for the complex frequency } \\
\text { shifted PML parameters }\end{array}$ \\
\hline 2001 & Teixeira, et al., introduce a conformal PML for general coordinate frames \\
\hline 2001 & Ziolkowski and Heyman develop FDTD models for negative metamaterials \\
\hline 2002 & Chavannes develops stable multi-nested sub-grid modeling method \\
\hline 2004 & Akyurtlu and Werner develop an FDTD model for bi-anisotropic media \\
\hline 2004 & $\begin{array}{c}\text { Chang and Taflove combine FDTD with a quantum mechanical atomic model to } \\
\text { simulate a four-level atomic system }\end{array}$ \\
\hline 2005 & FDTD modeling of photonic nanojets from nanoparticles $(\mathrm{Li}$, et al) \\
\hline $2006-2007$ & Ong, et al., use FDTD to simulate solar cells with nanotube array cathodes \\
\hline 2007 & Zhao, et al., use FDTD to model "spatially" dispersive media \\
\hline 2008,2009 & $\begin{array}{l}\text { Using the FDTD method, Kong, et al., showed that subwavelength pits in a metal } \\
\text { substrate can be used for high-density optical data storage }\end{array}$ \\
\hline 2010 & Argyropoulos, et al., use FDTD to model the "optical black hole" \\
\hline
\end{tabular}

\subsubsection{Maxwell's Equations}

Table 2.14 Quantities and coefficients contained within Maxwell's equations. Note $\mu_{0}=4 \pi \times 10^{-7} \mathrm{~N} / \mathrm{A}^{2}[194]$.

\begin{tabular}{|l|l|}
\hline$E=$ electric field & $M=$ magnetization \\
\hline$B=$ magnetic induction intensity & $i=$ electric current \\
\hline$H=$ magnetic field strength & $J=$ current density \\
\hline$D=$ electric displacement & $c=$ speed of light \\
\hline$\rho=$ charge density & $P=$ polarization \\
\hline$\varepsilon_{0}=$ free-space electric permittivity & $\sigma=$ electrical conductivity \\
\hline$\mu_{0}=$ free-space magnetic permeability & \\
\hline
\end{tabular}


Maxwell's equations in integral form in the absence of magnetic or polarizable media:

$\oint \vec{E} \cdot d \vec{A}=\frac{q}{\varepsilon_{0}}$

Gauss' Law for Electricity

$\oint \vec{B} \cdot d \vec{A}=0$

Gauss' Law for Magnetism

$\oint \vec{E} \cdot \vec{d} s=-\frac{d \phi_{B}}{d t}$

Faraday's Law of Induction

$\oint \vec{B} \cdot \vec{d} s=\mu_{0} i+\frac{1}{c^{2}} \frac{\partial}{\partial t} \int \vec{E} \cdot d \vec{A}$

Ampere's Law

Maxwell's equations in differential form in the absence of magnetic or polarizable media:

$\nabla \cdot \mathrm{E}=\frac{\rho}{\varepsilon_{0}}=4 \pi k \rho$

Gauss' Law for Electricity

$\nabla \cdot \mathrm{B}=0$

Gauss' Law for Magnetism

$\nabla \times \mathrm{E}=-\frac{\partial B}{\partial t}$

Faraday's Law of Induction

$\nabla \times \mathrm{B}=\frac{4 \pi k}{c^{2}} J+\frac{1}{c^{2}} \frac{\partial E}{\partial t}=\frac{J}{\varepsilon_{0} c^{2}}+\frac{1}{c^{2}} \frac{\partial E}{\partial t}$

Ampere's Law

where $k=1 / 4 \pi \varepsilon_{0}$ (i.e., Coulomb's constant) and $c^{2}=1 / \mu_{0} \varepsilon_{0}$. Note that $\nabla \cdot$ and $\nabla \times$ represent the vector operations divergence and curl, respectively [194]. Maxwell's equations in differential form in the presence of magnetic or polarizable media:

$\nabla \cdot \mathrm{D}=\rho$

Gauss' Law for Electricity 
where $D=\varepsilon_{0} E+P$ (general case), $D=\varepsilon_{0} E$ (free space), and $D=\varepsilon E$ (isotropic linear dielectric) [194].

$\nabla \cdot \mathrm{B}=0$

Gauss’ Law for Magnetism

$\nabla \times \mathrm{E}=-\frac{\partial B}{\partial t}$

Faraday's Law of Induction

$\nabla \times \mathrm{H}=J+\frac{\partial D}{\partial t}$

Ampere's Law

where $B=\mu_{0}(H+M)$ (general case), $B=\mu_{0} H$ (free space), and $B=\mu H$ (isotropic linear magnetic medium) [194]. Here again, $\nabla \cdot$ and $\nabla \times$ represent the vector operations divergence and curl, respectively [194]. The basic principle of FDTD is to numerically solve Maxwell's differential equations:

$\nabla \times \overrightarrow{\mathrm{E}}=-\frac{\partial \vec{B}}{\partial t}$

$\nabla \times \overrightarrow{\mathrm{H}}=\vec{J}+\frac{\partial \vec{D}}{\partial t}$

where:

$\vec{D}=\varepsilon \vec{E}$

$\vec{B}=\mu \vec{H}$

$\vec{J}=\sigma \vec{E}$ 


\section{Chapter 3}

\section{FDTD Simulation Methodology}

Chapter 3 summarizes the background of the FDTD method and each of the critical software settings including the simulation region settings, boundary conditions, mesh refinement options, excitation source settings and specifications, monitor types and configurations. Additionally, background on the Fabry- Pérot interferometer simulated as an introductory problem using FDTD is discussed as well as supplementary information such as dipole source polarization, the skin depths of metals, the Poynting vector, and select cavity simulation results. Following the Fabry- Pérot simulation results is the presentation of the initial plasmonic lattice parameter optimization methodology. 


\subsection{FDTD Background}

The FDTD method was proposed by Kane S. Yee in 1966 [190, 193]. Yee proposed discrete solutions to Maxwell's equations based on central difference approximations of spatial and temporal derivatives of the curl equations. In his work, Yee staggered the $E$ - and $H$-fields in both space and time in order to obtain second order accuracy [190, 193]. Subsequently, Yee derived a three-dimensional formulation that he later used to validate two-dimensional problems [190, 193]. For nearly a decade Yee's method went unnoticed until Taflove and Brodwin in 1975 used his approach to simulate biological heating and light scattering by dielectric cylinders [190]. Due to continuous advances in computer technology, FDTD has seen persistent growth since its founding in the late 1970s [190]. Factors driving the significant growth of FDTD in both academia and industry include its fast algorithm and overall simplicity [190]. The fast algorithm of FDTD lies in the following: if there are $N$ degrees of freedom in a three-dimensional space, each iteration in time only requires $0(N)$ floating-point operations. This introduces a caveat whereby the discrete mesh must fill the full three-dimensional space and, therefore, $N$ grows cubically with linear dimension(s) within the domain of interest [190]. Despite this aforementioned caveat, the method is extremely useful in solving sophisticated engineering problems.

FDTD is currently the most popular method in computing EM phenomena seeing extensive use in modeling, solving, and studying of Maxwell's equations across multiple complex geometries [189-192]. Due to its accuracy, simplicity, and versatility, FDTD has been successfully applied to a broad scope of simulation applications such as antennas, CMOS image sensors, diffractive optics, PhCs, graphene, integrated photonic components, liquid crystals and organic light-emitting diodes (OLEDs), metal and/or dielectric-induced light scattering, metamaterials, microstrip circuits, solar cells/photovoltaics, surface metrology, and plasmonics [190-192, 196]. Computational electromagnetics can be divided based on the domain (either frequency or time) in which Maxwell's equations are being explored. Time domain computational methods include FDTD and transmission line matrix (TLM) while frequency domain methods include finite element method (FEM), finite difference frequency domain (FDFD), method of moments (MoM), and the fast multipole method (FMM) [191]. FDTD has been commonly used as an optical solver of Maxwell's equations specifically regarding dipole point sources, the Gaussian beam, wave optics, and ray optics for feature sizes on the order of wavelength [192]. 
In FDTD, both space and time are divided into discrete segments. Space is divided up into box-shaped cells (i.e., the Yee cell - the basis for any FDTD simulation) with the E-field components located at the edges of the cell with the $H$-field components positioned on the faces [192-193, 195-196]. The field orientation of the Yee cell is such that every $E$ component is surrounded by four $H$ components and every $H$ component is surrounded by four $E$ components [192-193, 195-196]. Figure 3.1 delineates the Yee cell showing the locations of the $E$ and $H$-field components.

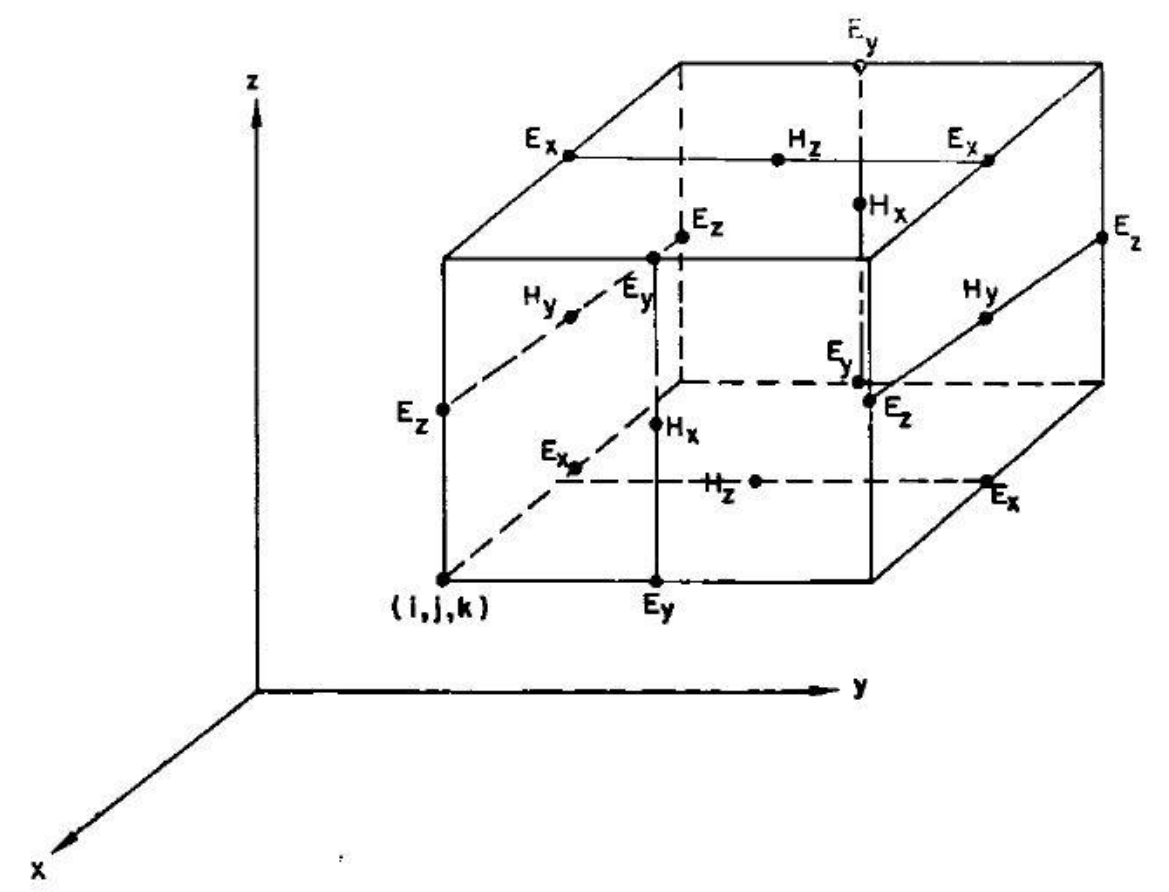

Figure 3.1 The Yee cell showing the positions of the various field components with the $E$-field components located at the points of the cell with the $H$-field components located in the center of each face of the cell [193].

Time is quantized into small steps (i.e., the time-step setting in FDTD simulations) representing the amount of time required for the field to travel from one Yee cell to the next, referred to as the Courant stability criterion [195-197]. The Courant stability criterion states that, for stability reasons, a field component cannot propagate more than one cell size in the time-step $(\Delta t)$ :

$\Delta t \leq \frac{\Delta z}{c_{0}}$ 
since the wave travels at the speed of light $\left(c_{0}\right)$. The above stability condition solely applies to onedimensional problems [197]. It can be proven that, in general, the Courant condition is given by:

$\Delta t \leq \frac{\Delta}{c \sqrt{d}}$

with $d=1,2,3$ for one-, two-, or three-dimensional problems, respectively, and $\Delta$ is the smallest Yee cell size [197]. A common choice for the time step in one-, two-, and three-dimensional cases:

$\Delta t=\frac{\Delta}{2 c_{0}}$

The FDTD method discretizes Maxwell's equations in both space and time in order to find the $E$ and $H$ fields at different locations in space and steps in time [195]. Furthermore, FDTD can be conveniently applied to simulating EM scattering and radiation from a particle of complex shape as well as non-uniform dielectric objects by adjusting the number, size, and other properties of the Yee cell [195]. In a Cartesian coordinate system, (136) and (137) are equivalent to the following scalar equations:

$$
\begin{aligned}
& \frac{\partial E_{z}}{\partial y}-\frac{\partial E_{y}}{\partial z}=-\mu \frac{\partial H_{x}}{\partial t} \\
& \frac{\partial E_{x}}{\partial z}-\frac{\partial E_{z}}{\partial x}=-\mu \frac{\partial H_{y}}{\partial t} \\
& \frac{\partial E_{y}}{\partial x}-\frac{\partial E_{x}}{\partial y}=-\mu \frac{\partial H_{z}}{\partial t} \\
& \frac{\partial H_{z}}{\partial y}-\frac{\partial H_{y}}{\partial z}=\varepsilon \frac{\partial E_{x}}{\partial t}+\sigma E_{x} \\
& \frac{\partial H_{x}}{\partial z}-\frac{\partial H_{z}}{\partial x}=\varepsilon \frac{\partial E_{y}}{\partial t}+\sigma E_{y} \\
& \frac{\partial H_{y}}{\partial x}-\frac{\partial H_{x}}{\partial y}=\varepsilon \frac{\partial E_{z}}{\partial t}+\sigma E_{z}
\end{aligned}
$$


Using the function $f(x, y, z, t)$ to denote the $E$ or $H$ field in the Cartesian coordinate system, the following form can be obtained:

$f(x, y, z, t)=f(\mathrm{i} \Delta x, \mathrm{j} \Delta y, \mathrm{k} \Delta z, \mathrm{n} \Delta t)=f^{n}(i, j, k)$

Then, $f(x, y, z, t)$ can be discretized via the central difference approximation in both space and time yielding the following expressions:

$\frac{\partial f(x, y, z, t)}{\partial x} \approx \frac{f^{n}\left(i+\frac{1}{2}, j, k\right)-f^{n}\left(i-\frac{1}{2}, j, k\right)}{\Delta x}$

for $x=i \Delta x$

$\frac{\partial f(x, y, z, t)}{\partial y} \approx \frac{f^{n}\left(i, j+\frac{1}{2}, k\right)-f^{n}\left(i, j-\frac{1}{2}, k\right)}{\Delta y}$

for $y=j \Delta y$

$\frac{\partial f(x, y, z, t)}{\partial z} \approx \frac{f^{n}\left(i, j, k+\frac{1}{2}\right)-f^{n}\left(i, j, k-\frac{1}{2}\right)}{\Delta z}$

for $z=k \Delta z$

$\frac{\partial f(x, y, z, t)}{\partial t} \approx \frac{f^{n+\frac{1}{2}}(i, j, k)-f^{n-\frac{1}{2}}(i, j, k)}{\Delta t}$

for $t=n \Delta t$

The above sampling method of the EM field components contained within the Yee cell is most favorable to the differential calculation of Maxwell's equations and is consistent with Faraday's law of magnetic induction [195]. The size of the Yee cell is the most important constraint for obtaining an accurate field distribution in an FDTD simulation and must be significantly smaller than the smallest excitation wavelength [195]. A general consideration in specifying size Yee cell size is typically 10 cells per wavelength, or cells $\leq 1 / 10 \lambda$ of the shortest excitation wavelength of interest [195]. Due to computational limitations, the size of the cell cannot be infinitely small. Therefore, the cell size selected must consider computational resources available and the requisite calculation accuracy [195]. Section 3.2 details the simulation settings of Lumerical, Inc.'s FDTD simulation software used within the scope of this research. 


\subsection{FDTD Software Settings}

Lumerical's FDTD Solutions contains a wide array of settings spanning every aspect of the simulation environment. The following subsections detail noteworthy software settings that must be properly configured for obtaining the most accurate results possible. All subsequent information regarding Lumerical FDTD software settings is referenced directly from Lumerical's Knowledge Base [192].

\subsubsection{Simulation Region}

The simulation region contains three general settings: 1) dimension - two- or threedimensional simulation environment, 2) background index - the refractive index of the surrounding medium of the simulation region, and 3) simulation time - the maximum duration of the simulation being performed. Additionally, the size of the simulation region can be specified geometrically in $x, y$, and $z$ including center position, minimum and maximum position, and span (minimum position to maximum position). Within the specified geometric constraints of the simulation region exist supported boundary conditions that define the properties of the simulation region at its boundaries.

\subsubsection{Boundary Conditions}

Boundary conditions supported by Lumerical's FDTD software will now be discussed.

1. Perfectly Matched Layer (PML): PML boundaries absorb EM waves incident upon them effectively simulating an "open", reflection-less boundary. Moreover, the PML is an artificial material which attempts to match the impedance of the adjacent materials to prevent reflection while also absorbing any light that is incident. Lumerical FDTD allows the user to specify all of the parameters that control the absorption properties of the PML including the number of PML layers and PML profile (custom, stabilized, standard, or steep angle). The custom PML profile allows the user to specify every setting of the PML, the stabilized PML profile contains 42 layers, the standard PML profile contains 8 layers, and the steep angle profile contains 12 layers. Typically, the standard PML profile is sufficient for use in most simulations. The stabilized PML profile is used primarily as a troubleshooting option for simulations that diverge (i.e., simulation instability either due to the $d t$ stability factor or the PML itself). 
The steep angle PML profile is best suited for use in cavity problems where light can hit the PML boundaries at very sharp, or steep, angles. Unlike conventional boundary conditions, PML boundaries have a finite thickness (i.e., they occupy a finite volume surrounding simulation region). It is within this volume where light absorption happens.

- LAYERS: For discretization purposes, the PML regions are divided into layers.

- KAPPA, SIGMA, ALPHA: The absorption properties of the PML regions are controlled by three parameters. Kappa is unitless by definition, but sigma and alpha must be entered into the PML settings table as normalized unitless values. Kappa, sigma and alpha are all graded inside the PML regions using polynomial functions. Parameter alpha is sometimes described as a complex frequency shift (CFS) in the literature whose primary function is to improve numerical stability. Increasing the ratio alpha/sigma will make a PML boundary more stable but reduces its absorption effectiveness and is why the stabilized profile requires a greater number of layers. To recover the SI unit values of alpha and sigma, it is necessary to multiply by twice the permittivity of free space and divide by the time-step employed in the simulation.

- POLYNOMIAL: Specifies the order of the polynomial used to grade kappa and sigma.

- ALPHA POLYNOMIAL: Specifies the order of the polynomial used to grade alpha.

- MIN LAYERS, MAX LAYERS: Enforces a sensible range of values for the number of PML layers. During a given simulation, the EM fields are calculated both within the simulation area and within the boundary condition area. Since the fields are still being propagated in the boundary conditions, it is important that the material properties are also defined here (i.e., the reason why, by default, structures are extended through the PML). If the "extend structure through PML" option is selected, it will extend any structures that touch the inner PML boundary in the direction normal to the boundary. An interface in this area will act like any other interface and cause reflections. 
All structures should extend completely through the boundary condition area to minimize these reflections and is especially important when using many PML layers. As more layers of PML are added, the boundary condition area will become larger.

2. Metal: metal boundary conditions are used to specify boundaries that behave as a perfect electric conductor (PEC). In this boundary condition, the component of the $E$ field parallel to the metal boundary is zero and the component of the $H$-field perpendicular to the metal boundary is zero. Metal boundaries are perfectly reflecting allowing for no energy to escape the simulation volume along the boundary.

3. Perfect Magnetic Conductor (PMC): PMC boundary conditions are the magnetic equivalent to metal (PEC) boundary conditions. The component of the $H$-field parallel to a PMC boundary is zero and the component of the $E$-field perpendicular to the PMC is zero.

4. Periodic: periodic boundary conditions are used when both the fields and the structures are periodic. This boundary condition can be employed in one or more directions to simulate a structure(s) periodic in one direction, but not necessarily in other $\operatorname{direction}(\mathrm{s})$.

5. Antisymmetric/Symmetric: antisymmetric/symmetric boundary conditions are used in simulations that exhibit one or more planes of symmetry - both the structure and source must be symmetric. Antisymmetric boundary conditions are anti-mirrors for the $E$-field and mirrors for the $H$-field. Symmetric boundary conditions act as mirrors for the $E$ field and anti-mirrors for the $H$-field.

The boundary condition tab contains the following options:

- $\quad X M I N$, XMAX, YMIN, YMAX, ZMIN, ZMAX BOUNDARIES: These fields describe the boundary conditions to be applied along the perimeter of the simulation region. Symmetric and asymmetric boundary conditions should be applied to the lower boundary conditions. 
- ALLOW SYMMETRY ON ALL BOUNDARIES: By default, symmetric and antisymmetric conditions can only be used on the lower boundaries ( $x$ min, $y$ min and $z$ min). This box allows you to also use symmetry and anti-symmetric conditions on the upper boundaries in order to simulate periodic structures that exhibit symmetry.

- PML SETTINGS - TYPE: Sets the type of PML boundary formulation to be used. The options are a PML based on a stretched coordinate formulation or a PML based on a uniaxial anisotropic material formulation (i.e., the legacy option).

- PML SETTINGS - SAME SETTINGS ON ALL BOUNDARIES: When unchecked, this option allows users to set different PML settings for the XMAX, YMIN, YMAX, ZMIN and ZMAX boundaries. When checked, the same PML settings are used for all boundary conditions.

- PML SETTINGS - TABLE: Sets the PML profile to be used on each boundary.

\subsubsection{Simulation Region Mesh Settings}

By default, an automatically generated, graded mesh is used in regions where no mesh overrides are present. The mesh step size of the automatic mesh is based on the mesh accuracy setting specified under the Boundary Conditions tab of the FDTD solver region object. Three types of mesh generation algorithms are available in Lumerical FDTD:

1. Auto Non-Uniform: default setting where a non-uniform mesh is automatically generated based on the mesh accuracy slider bar. It is strongly recommended to start with a mesh accuracy of 1-2 for faster initial run times.

Higher mesh accuracies can be used for convergence testing if necessary. The MESH ACCURACY parameter is an integer from 1-8, where 1 is the lowest accuracy and 8 is highest accuracy (i.e., a finer mesh). Many factors go into the meshing algorithm, including source wavelength, material properties and structure geometry. The number of mesh points per wavelength (ppw) is a major consideration for the meshing algorithm. Accuracy 1 corresponds to a target of $6 \mathrm{ppw}$. Acc 2 corresponds to $10 \mathrm{ppw}$, Acc 3 corresponds to $14 \mathrm{ppw}$, and so on in increments of $4 \mathrm{ppw}$ per point on the slider bar. 
It is important to remember that wavelength is inversely proportional to the refractive index. In high index materials, the effective wavelength is smaller, meaning that the meshing algorithm will use a smaller mesh for higher index materials.

2. Custom Non-Uniform: this setting allows additional options for customizing how the non-uniform mesh is generated. If setting the mesh cells using wavelength, the default setting of 10 is generally sufficient, but may be reduced to 6-8 for coarse simulations. The grading factor determines the maximum rate at which the mesh can be modified. The grading factor should be between 1 and 2 and is $\sqrt{2}$ by default.

3. Uniform: a uniform mesh is applied to the entire simulation volume regardless of any material properties. If a mesh override region is used in conjunction with this option, the override region will force the mesh size everywhere not just within the override region (since the mesh is uniform).

The following time-step settings are also contained within the simulation region mesh settings:

- DT STABILITY FACTOR: A setting which determines the size of the time-step used during the simulation, defined as a fraction of the Courant numerical stability limit. A larger number will result in faster simulation times, and a smaller number will result in slower simulation times. The Courant stability condition requires that this setting must be less than 1 for the FDTD algorithm to remain numerically stable.

- DT: The time-step of the FDTD/Propagator simulation determined by the values of the spatial grid to ensure numerical stability (not user definable).

\subsubsection{Mesh Refinement Options}

Mesh override regions can be used in regions where a finer mesh than the automatically generated mesh is desired such as when attempting to accurately resolve the thickness of a layer. Lumerical's Conformal Mesh Technology (CMT) employs a rigorous physical description of Maxwell's integral equations near the interface between two materials capable of incorporating Lumerical's Multi-Coefficient Materials (MCMs). At optical frequencies, the dispersive nature of commonly used materials must be considered. Lumerical's MCMs allows for the simulation of highly dispersive materials used in applications including biosensors, CMOS image sensors, and solar cells. 
Lumerical's CMT can handle interfaces between arbitrary dispersive media generally providing greater accuracy for a given mesh size making it possible to run simulations much faster without sacrificing accuracy. Due to the $1 / d x^{4}$ dependence of the simulation time on the mesh size, results can often be achieved in roughly 1/10 the time. While FDTD is ideally suited in principle to provide broadband performance data for nanoscale photonic devices, the standard Yee cell FDTD algorithm relies on discretizing the underlying structure onto a Cartesian mesh. The resulting discretized structure is unable to account for structure variations that occur within any single Yee cell, resulting in a "staircase" permittivity mesh coinciding with the Cartesian mesh. In general, conformal mesh methods try to account for sub-cell features by solving Maxwell's integral equations near structure boundaries. As an extension of general conformal mesh algorithms to handle dispersive materials, it is easily possible to generate more simple conformal mesh models like the Yu-Mittra model from Lumerical's CMT. Lumerical's CMT is capable of generating significant accuracy improvements relative to staircasing results that can be illustrated by applying the CMT to a multilayer stack - a common element incorporated within various photonic designs. Also, the CMT provides sub-mesh sensitivity to changes in geometrical parameters greatly facilitating design optimization. Owing to its inherent compatibility with Lumerical's MCMs, CMT allows designers to more efficiently prototype broadband nano-scale photonic design concepts in high index contrast and dispersive materials. Mesh refinement options contained within Lumerical FDTD are as follows:

1. Conformal Variant 0: CMT is not applied to interfaces involving metals or PECs.

2. Conformal Variant 1: CMT is applied to all materials including metals and PECs.

3. Conformal Variant 2: The Yu-Mittra method 1 is applied to interfaces involving metals and PECs with CMT applied to all other interfaces.

4. Yu-Mittra Method 1: Introduced by Yu and Mittra to provide greater accuracy when modeling PEC/dielectric interfaces. Lumerical's formulation is a slight extension of the original Yu-Mittra formulation that can be used with arbitrary dispersive media. In Figure 3.2 below, the original formulation where the presence of a PEC is considered by reducing the contour integral $C$ to include only the region outside the PEC where the $E$-field is non-zero $(C 1)$. In Lumerical's implementation, when updating the $B$-field, the contour $C 1$ is evaluated in material 1 , while the contour $C 2=(C-C 1)$ is evaluated in material 2. 
In the event that one of the materials is a PEC (where $E=0$ ), this is identical to the original $\mathrm{Yu}$-Mittra formulation for PEC. If more than two materials are found in a single cell, the method reverts to Staircasing for that cell.

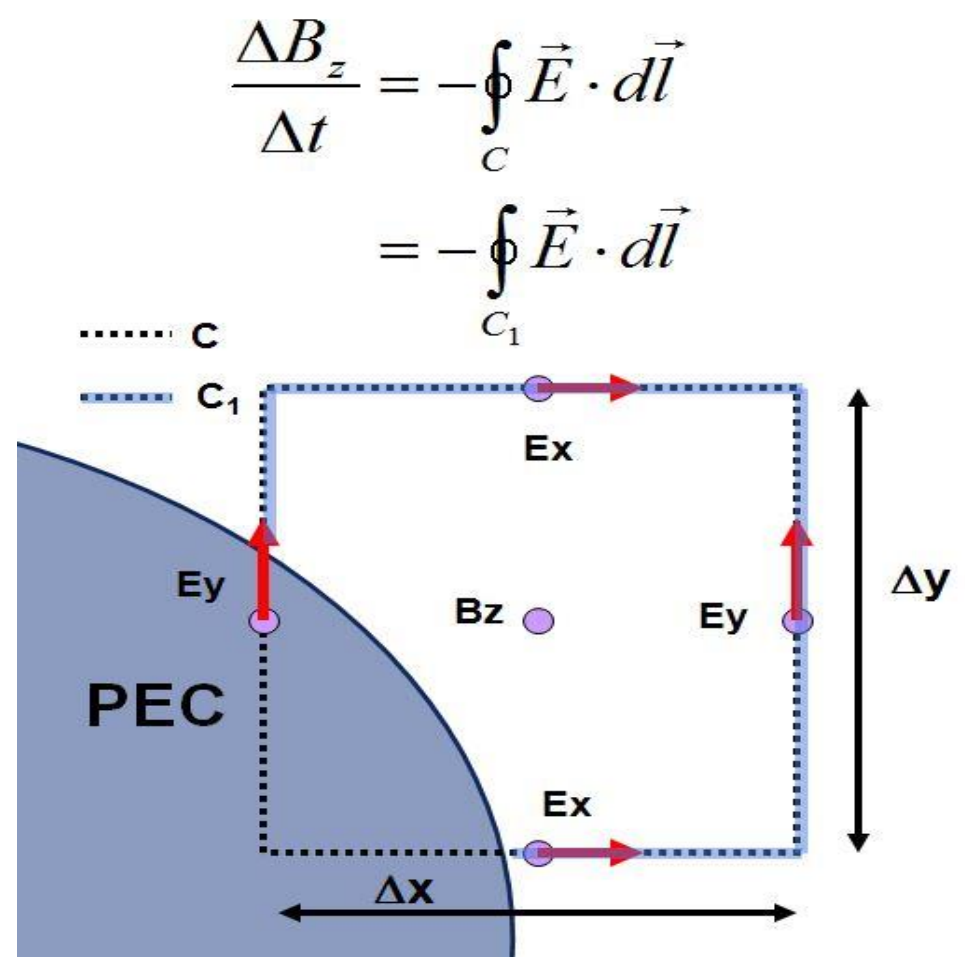

Figure 3.2 Yu-Mittra method one. The presence of a PEC is accounted for by reducing the contour integral $C$ to include only the region outside the PEC where the $E$-field in non-zero [192].

5. Yu-Mittra Method 2: Introduced by Yu and Mittra to provide greater accuracy when modeling dielectric interfaces. Lumerical's formulation is a slight extension of the original Yu-Mittra method that can be used with arbitrary dispersive media. An effective permittivity is assigned to each permittivity component in the Yee cell weighted by the fraction of the mesh step that is inside material one or material two. If more than two materials are found in a single cell, the method reverts to Staircasing for that cell.

6. Staircasing: The material at each position of the Yee cell is evaluated to determine which material it is in, and the $E$-field at that location uses only that single material property. 
The resulting discretized structure is unable to account for structure variations that occur within any single Yee cell, resulting in a "staircase" permittivity mesh that coincides with the Cartesian mesh. Furthermore, any layers are effectively moved to the nearest $E$-field position on the Yee cell, meaning that layer thickness cannot be resolved to better than $d x$.

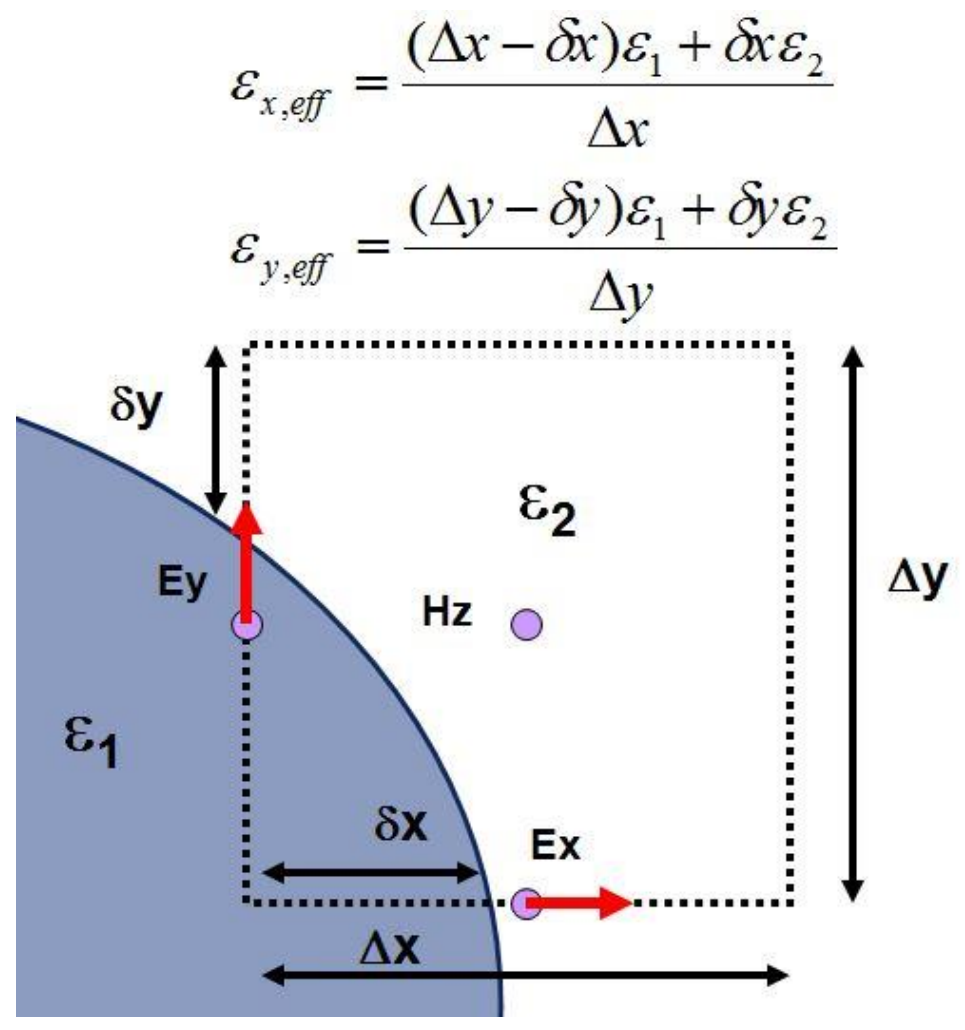

Figure 3.3 Yu-Mittra method two. An effective permittivity is assigned to each permittivity component in the Yee cell weighted by the fraction of the mesh step inside material one or material two. If more than two materials are found in a single cell, the method reverts to Staircasing for that cell [192].

The Yu-Mittra method is illustrative of the approach used by all conformal meshing methods that can be used to improve accuracy when modeling curved PEC surfaces. While this method is simple to implement and accurately describes the interaction of EM radiation at microwave and radio frequencies with most metallic materials it does not provide accurate results for many types of simulations conducted at optical frequencies. In addition to the user-definable simulation region and mesh refinement settings, an appropriate EM source must be chosen and properly configured. 


\subsubsection{Source Types and Specifications}

Lumerical FDTD software is capable of incorporating multiple EM sources into the simulation environment. The following explores common EM sources relevant to the nanophotonic simulations performed in this work:

1. Dipole (Point) Sources - Oscillating dipoles act as sources in Maxwell's equations for producing EM fields. Their position and direction are both specified in terms of their center position and orientation via the angles theta $(\theta)$ and phi $(\phi)$. The orientation of the dipole source is partially dependent upon the polarization of the propagator simulation (i.e., TE or TM). General and frequency/wavelength dipole source specifications are detailed below:

- DIPOLE TYPE: A pull-down menu in which the point source can be configured as an oscillating point charge (electric dipole) or a current loop (magnetic dipole). The radiation patterns of these dipoles are similar, but not identical.

- AMPLITUDE: Source amplitude whose units depend on the type of dipole source.

- BASE AMPLITUDE: This is the amplitude that will generate a radiated CW power of $10 \mathrm{nW} / \mathrm{m}$ in two-dimensional simulations and $1 \mathrm{fW}$ in three-dimensional simulations.

- TOTAL AMPLITUDE: The product of the AMPLITUDE and the BASE AMPLITUDE - this is the amplitude actually used in simulation.

- PHASE: The phase of the point source measured in degrees. This setting is only useful for setting relative phase delays between multiple radiation sources.

- THETA $(\theta)$ : The angle with respect to the $z$-axis of the dipole vector

- $P H I(\phi)$ : Angle with respect to positive $x$-axis of the dipole vector.

The Frequency/Wavelength tab can be accessed through the individual source properties or the global source properties. Note that the plots on the right-hand side of the window update with updates to the parameters. The window contains three figures: 1) the top figure shows wavelength content, 2) the middle figure shows frequency content, and 3) the bottom figure shows the temporal content of the source settings. 
The time domain settings can be modified with full consideration of the following temporal attributes:

- PULSE DURATION: Choose a pulse duration that can accurately span your frequency or wavelength range of interest. Note that very short pulses contain many frequency components and, therefore, rapidly disperse. Consequently, short pulses require more points per wavelength for an accurate simulation.

- PULSE OFFSET: This parameter defines the temporal separation between the start of the simulation and the center of the input pulse. To ensure that the input pulse is not truncated, the pulse offset should be at least two times the duration of the pulse. Doing this will ensure that the frequency distribution around the center frequency of the source is close to symmetrical, and the initial fields are close to zero at the beginning of the simulation.

- SOURCE TYPE: In general, standard and broadband source types can be selected. Standard sources consist of a Gaussian pulse at a fixed optical carrier, while the broadband sources consist of a Gaussian pulse with a varying optical carrier across the pulse envelope. Broadband sources can be used to perform simulations in which wideband frequency data is required; this type of frequency range cannot be accurately simulated using the standard source type.

- BANDWIDTH: The FWHM frequency width of the time domain pulse.

- ELIMINATE DISCONTINUITY: Enabled by default, ensures the function has a continuous derivative (i.e., smooth transitions from/to zero) at the start and end of a user defined source time signal.

- OPTIMIZE FOR SHORT PULSE: Use the shortest possible source pulse. This option is enabled by default that should only be disabled when it is necessary to minimize the power injected by the source that is outside of the source range (e.g., convergence problems related to broadband steep angled injection).

The following results are returned after a successful simulation run:

- DIPOLEPOWER: Returns the power injected into the simulation region by a dipole. The units will be in $\mathrm{W}$ if continuous wave $(\mathrm{CW})$ normalization is used and $\mathrm{W} / \mathrm{Hz}^{2}$ if no norm is used. CW normalization will be discussed later. 
- PURCELL: Returns the Purcell factor by utilizing the power measurement and the emission rate enhancement of a spontaneous emitter inside a cavity or resonator.

- TIME SIGNAL: Time domain signal of the source pulse.

- SPECTRUM: The Fourier transform of the time signal.

2. Plane Wave Sources - Plane wave sources are used to inject laterally uniform EM energy from one side of the source region. In two-dimensional simulations, the plane wave source injects along a line and injects along a plane in three-dimensional simulations. It is also possible to inject a plane wave at an angle. The plane wave source is identical to the Gaussian source, with the only difference being the SOURCE SHAPE setting.

Sources in FDTD Solutions always inject a pulse (i.e., broadband), even when the user is only interested in collecting data at a single frequency. Pulsed sources are more efficient for collecting broadband information and are at least as efficient when collecting information at a single frequency. Therefore, there is no reason to use $\mathrm{CW}$ sources. The $\mathrm{CW}$ response of a structure can easily be obtained from a simulation that used a pulse source. To understand CW normalization, one must remember that FDTD is a time domain method whereby the EM fields are calculated as a function of time. In an FDTD simulation, the system is excited by some EM source (dipole, Cauchy/Lorentzian beam, Gaussian beam, plane wave, etc.) and the time signal of the source $(s(t))$ is a pulse. As an example, this source pulse could be:

$s(t)=\sin \left(\omega_{0}\left(t-t_{0}\right)\right) e^{\left(-\frac{\left(t-t_{0}\right)^{2}}{2(\Delta t)^{2}}\right)}$

whose Fourier transform is:

$s(\omega)=\int e^{(i \omega t)} s(t) d t$

Ideally, $s(t)$ would be a dirac delta function where $s(\omega)=1$ allowing one to obtain the response of the system at all frequencies by running a single simulation. Consequently, it is more efficient and numerically accurate to excite the system with a short, chirped pulse such that the spectrum $\left(|s(\omega)|^{2}\right)$ is reasonably large in value over all frequencies of importance. 
Without CW normalization (i.e., "nonorm"), the power and profile monitors return the response of the system to the simulated input pulse $s(t)$ :

$$
\vec{E}_{\text {sim }}(\omega)=\int e^{(i \omega t)} \vec{E}(t) d t
$$

The simulated $E$-field as a function of angular frequency $\left(E_{\text {sim }}(\omega)\right)$ depends on both the source pulse used $(s(t))$ and the system being studied. With CW normalization, the power and profile monitors return the impulse response of the system:

$$
\vec{E}_{i m p}(\omega)=\frac{\vec{E}_{s i m}(\omega)}{s(\omega)}
$$

The impulse response of the system is of greater significance because it is completely independent of the source pulse used in the excitation of the simulated system. Exemplified below is a beam source injected into free space at $z=z_{0}$ with the source time signal from (155). The $E$-field at the source injection plane takes the following form:

$$
E\left(x, y, z_{0}, t\right)=E_{0}\left(x, y, z_{0}\right) s(t)
$$

The field returned via CW normalization is the field that would exist if a CW source of amplitude $E_{0}$ had been used at the angular frequency $(\omega)$. Any frequency dependence is removed due to the finite pulse length of the excitation source possessing returned fields in the same units as the time domain fields:

$E(x, y, z, \omega)=E_{0}(x, y, z)$

By default, the simulation run time is set to $1,000 \mathrm{fs}$. In order to get accurate frequency domain monitor results, enough optical cycles are required for the full source pulse to be injected, for the fields to propagate through the simulation region, and for those fields to fully decay. If the fields in the time signal are truncated suddenly when the simulation ends early, artifacts in the Fourier transform used to obtain frequency domain results can result. 


\subsubsection{Monitor Types and Data Returned}

Lumerical FDTD has three monitors available for collecting simulation data: 1) time domain monitors, 2) frequency domain EM field monitors, and 3) absorption monitors.

1. Time Domain Monitors - Provide time domain information for fields components over the course of the simulation run-time. Time domain monitors can consist of point, surface, or volume geometries to capture temporal information over different spatial extents contained within the simulation region.

2. Frequency Domain EM Field Monitors - Collect CW, steady-state EM field data in the frequency domain within the simulation region.

3. Absorption Monitors - Directly return information about total absorption and absorption distribution within the monitor volume. The data collected is normalized to the power injected into the simulation region (i.e., source power).

Another useful feature in Lumerical FDTD is monitor apodization. Monitor apodization allows for the exclusion of effects that occur near the start and/or end of a simulation run from the monitor's Fourier transform. Any three settings of this option can be useful in cavity resonator problems where resonance takes a long time to decay (i.e., filtering away short-lived transients that occur when the system is excited with a dipole source). Monitor apodization applies a window function to simulation fields $(E(t))$ before the monitor performs its Fourier transform to obtain $E(\omega)$; this makes it possible to calculate $E(\omega)$ from a portion of the time signal. In order to avoid the sudden truncation of the fields in the time signal, monitor apodization can be utilized. Apodization, in general, will invalidate any source normalization performed and is therefore not suitable for accurate power or field intensity measurement. This setting applies a filter in the time domain to gradually ramp down the amplitude of the fields removing the need to run the simulation for the full time that it takes for the fields to completely decay from the simulation volume. Figure 3.4 details all three apodization settings available in Lumerical's FDTD. 


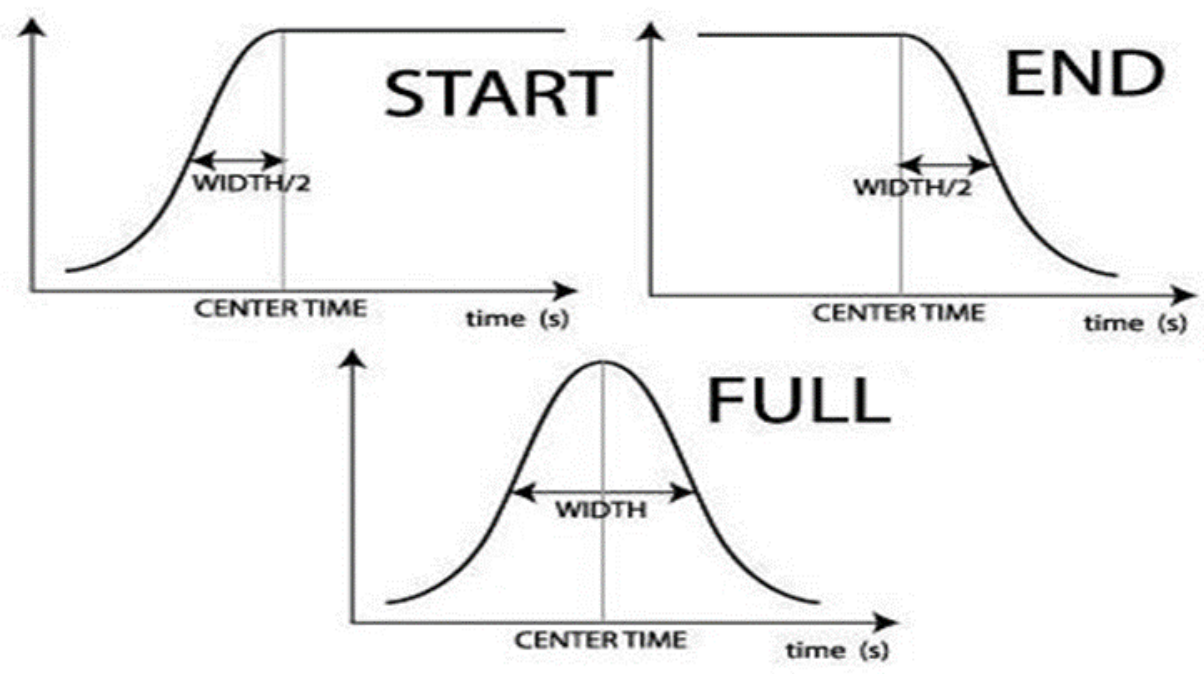

Figure 3.4 Start (left), End (right), and Full (center bottom) monitor apodization options in Lumerical FDTD [192].

Section 3.3 presents the usage of Lumerical FDTD to model the Fabry-Pérot interferometer, or etalon - a 'simple' cavity problem initially undertaken in this research with the goal of gaining a more in-depth understanding of the software, its settings, and the physics behind the software results. Note, the knowledge obtained from the modeling and simulation of the FabryPérot interferometer were applied to the plasmonic lattice simulations performed later in Section 3.4 .

\subsection{The Fabry-Pérot Interferometer}

A Fabry- Pérot interferometer was first modeled in this research as a simple problem with the goal of comparing the resonance conditions of the cavities constructed with various materials aimed at understanding the physics of FDTD and interpreting the returned results.

\subsubsection{Theoretical Background}

Interferometry is a family of techniques based on the superposition of waves. Developed in 1899, the Fabry-Pérot interferometer makes use of multiple reflections between two closely spaced reflective surfaces applicable in astronomy, spectroscopy, and telecommunication [222]. A portion of the light is transmitted each time the light reaches the second surface which results in offset beams that can interfere with one another. 
The large number of interfering light rays produces an interferometer of high resolution similar to multiple slits of a diffractive grating that, when focused, forms an interference pattern of concentric circles [221-222]. According to Snell's law, when light travels from a less optically dense medium into a more optically dense medium (i.e., from air to glass or a metallic mirror), it undergoes refraction:

$n_{1} \sin \left(\theta_{1}\right)=n_{2} \sin \left(\theta_{2}\right)$

In the Fabry-Pérot interferometer, once the light reaches the second mirror it splits into two fractions: 1) a smaller fraction transmitted through the mirror, and 2) a larger fraction reflected within the cavity region between the two mirrors [220-222]. During this process, two changes occur: 1) an amplitude change resulting from a split in the wave at the boundary whereby the amplitude is distributed between the reflected and transmitted waves $\left(A_{\text {incident }}>A_{\text {reflected }}>\right.$ $A_{\text {transmitted }}$, and 2) a phase change of $\lambda / 2$ of the reflected wave resulting from the incident wave reflecting off of the more optically dense medium (mirror) compared to the medium through which the light travels. Most of the light is reflected back toward the first mirror with only a small fraction being transmitted through the second mirror. This allows for a large quantity of subsequent reflections and transmissions between the two mirrors resulting in multiple interfering waves being focused onto a screen [220-222]. The focused light enables high resolutions in the interferometer whose resolution is:

$\frac{\lambda}{\Delta \lambda}=\frac{m \pi \sqrt{r}}{1-r}$

where $m$ is the order of interference (approximately $2 d / \lambda$ for small angles). Expanding on (162) shows the least separation of two spectral lines is:

$\Delta \lambda=\frac{\lambda(1-r)}{m \pi \sqrt{r}}$ 
This separation means that the Rayleigh criterion is satisfied by the two wavelengths [222]. The interferometer can also be characterized by its free spectral range, or the change in wavelength necessary to shift the fringe system by one fringe:

$\partial \lambda=\frac{\lambda^{2}}{2 d}$

The purpose of the mirrors, with surfaces characterized by a reflection coefficient $(r)$, is to create pathlength differences between the subsequently transmitted waves equal to:

$2 \overline{\mathrm{AB}}-\overline{\mathrm{CD}}=2 \mathrm{~d} \cos \alpha$

The multiple transmitted waves are focused through a lens onto a screen where they interfere. Interference occurs when two or more waves superimpose, and the amplitude of the waves and their corresponding positions at each point in space combine to form a singular wave. As previously mentioned, the interference (fringe) pattern is one of concentric circles comprised of light and dark bands. The intensity of the light bands is determined by the extent to which the waves constructively interfere. Complete constructive interference produces the brightest bands with partial constructive interference producing the darker bands [220-222]. A varying distance $(d)$ between the mirrors alters the observed fringe pattern. Relating the pathlength difference to the constructive interference patterns, one observes an intensity maximum of the reflecting light when the pathlength equals the integer number of wavelengths:

$2 d \cos (\alpha)=m \lambda$

Interpretation of (163) shows that the interferometer can be used to determine unknown wavelengths with a known gap distance and/or incident angle, or vice-versa, as well as whether or not the light source is monochromatic [222]. In summary, interferometers see use in industry and research for determining changes in refractive index, measuring small distances, and surface irregularities [221-222]. 
The Fabry-Pérot interferometer was recently used by the Laser Interferometer Gravitational-Wave Observatory (LIGO) to detect and measure gravitational waves produced by the merging of two black holes billions of years ago [221]. Small holes, of dimensions $l$, have been known as poor transmitters of acoustic, EM, or other disturbances in cases where $\lambda<<l$ [198]. Defined as the ratio between power transmitted and that incident upon the aperture, hole, or slit has a normalized transmittance $\left(T_{N}\right)$ that is, at best, of the order $l^{4} / \lambda^{4}$. It has been shown that periodic arrays of small apertures in a metallic film can lead to extraordinary enhancement of optical transmission arising from the excitation of particular modes of the metallic structures [223]. This aforementioned discovery has led to advances in applications including light harvesting, near-field microscopy, and sensing that directly benefit from concurrent enhancement of the $E$-field in the vicinity of the apertures [198, 223].

The physical mechanisms underlying the extraordinary transmission granted by periodic arrays of small apertures, particularly for EM waves, distinct and cooperative roles played by surface modes (i.e., SPPs), and Fabry-Pérot resonances have been well established. Conversely, the mechanisms behind enhanced optical transmission through single apertures, however, have not [198]. While utilization of geometric and plasmon resonances for enhancing optical transmission through small, isolated apertures has been considered extensively, a comprehensive physical framework connecting the various approaches has yet to emerge [224-232]. Proposed is a model coupled to an EM oscillator yielding perfect transmission $\left(T_{N} \sim \lambda^{2 /} l^{2}\right)$ in the absence of all radiative losses. Applied in this model are openings in the resonance cavity and aperture-induced localized states in the waveguide in conjunction with approaches to geometric resonances for which the resonant condition decreases with aperture size [198]. The model extends to open-boundary apertures by specifically considering optical transmission through a pair of pinholes in a twodimensional waveguide where a localized mode bound to the pinholes whose frequency is below that of the onset of the extended-mode continuum exists. When resonance between the incident wave and the localized mode occurs, perfect transmission results [198]. As is the case with funneling, the key resonance is of the Fabry-Pérot type. However, unlike funneling, transmittance does not decrease with a decrease in slit width but is identical to the incident power for arbitrarily small hole sizes [198]. 
The model considers a small aperture defined by a closed boundary contained within a conductive wall with an accompanying resonant system with natural frequency $(\Omega)$ to which the aperture is coupled. Focusing on EM waves, $m$ is taken to be the induced effective magnetic dipole of the opening and $H_{0}$ to be the tangential component of the time-harmonic magnetic field existing in the absence of the opening. Note, a similar criterion applies to the effective electric dipole arising when the boundary problem involves the normal $E$-field component [198]. Assuming a diagonal magnetic polarizability tensor and that the only effect of the coupling is an introduction of poles at $\omega \approx \pm \Omega$ :

$m=\frac{\Omega^{2} l^{3} H_{0}}{\omega^{2}-\Omega^{2}-i \omega / \tau}$

where $l$ is the length of the aperture, $\omega=2 \pi \mathrm{c} / \lambda$ is the angular frequency, and $\tau<\infty$ accounts for losses. Understood here is a time dependence $e^{-i \omega t}$ [198]. In the absence of other dissipation sources, the lifetime $(\tau)$ can be obtained by equating the power delivered via the magnetic field:

$P_{D}(\omega)=\frac{\operatorname{Re}\left(i \omega m^{*} H_{0}\right)}{2}$

to the power radiated by the dipole:

$P_{R}(\omega)=\frac{\omega^{4}|m|^{2}}{3 \pi c^{3}}$

Consistent with the general theory of small apertures [233], (169) gives $m \approx-l^{3} H_{0}$ for $\omega<<\Omega$ and, therefore, $P_{R} \propto l^{6} / \lambda^{4}[198]$. Thus, the known off-resonance expression, $T_{N} \sim \lambda^{4 /} l^{4}$, mentioned earlier is obtained. In the case where:

$\left|\omega^{2}-\Omega^{2}\right| \ll \frac{\omega}{\tau}$ 
the following quantities become:

$|m| \approx \Omega \tau l^{3} H_{0}$

and:

$\tau=\frac{3 \pi c^{3}}{2 \Omega^{4} l^{3}}$

Using the above values, the resulting radiated power is:

$P_{R}(\Omega)=\frac{3 c \lambda^{2} H_{0}^{2}}{16 \pi}$

which is comparable to that of a focused incident beam down to a spot size on the order of the wavelength [198]. The anticipated result $T_{N}(\Omega) \sim \lambda^{2 /} l^{2}$ is returned setting a fundamental transmittance boundary. As per Babinet's principle [234], it can be seen that the transmission enhancement and the enhanced cross-section of resonant antennas are closely related phenomena [198]. Similar to antennas, the ideal $(\lambda / l)^{6}$ enhancement over the condition $\omega \ll<$ will be significantly reduced due to conduction losses inherent to real metals [198].

The analysis done above for (173) accounts for the interaction between single openings and single plasmons and geometric resonances and, with a select few adjustments, for transmission through a pair of holes in a resonance cavity [198]. However, (173) is not applicable to cases involving extended waveguided modes that do not directly interact with the external field. Another interesting observation is that when $|m|$ doubles in value, $P_{R}$ increases by a factor of four if the aperture radiates into half space causing the reflected beam of a tightly focused EM wave to change its phase by a factor of $\pi$ [198]. The above arguments can be extended to transmission through a slotted parallel-plate waveguide. 
Considering wave transmission for slotted parallel plates where $d$ is the gap distance between the two plates and $\Delta$ is the half-width of the slots, the two-dimensional Helmholtz equation needed to be solved:

$$
\left(\frac{\partial^{2}}{\partial x^{2}}+\frac{\partial^{2}}{\partial z^{2}}+k^{2}\right) \phi=0
$$

In (174), $\phi$ is a physically representation of either one of the Cartesian components of the fields in Maxwell's equations, or the free-space quantum wave function in the Schrödinger equation where $k=2 \pi / \lambda$ where $\lambda$ is the vacuum or deBroglie wavelength [198]. Noting that the condition for perfect transmission requires $T_{N} \sim \lambda / \Delta$ the waveguide walls are assumed to be infinitesimally thin and made of a perfect electrical conductor (PEC) such that the components of the magnetic (electric) field perpendicular (parallel) to the plates boundary condition at the walls is $\phi=0$. Furthermore, $\phi$ is taken to embody the $y$ component of the $E$-field $\left(E_{y}\right)$. It has been shown in [235] that the TM case where the $E$-field is perpendicular to the axis of interest does not result in perfect transmission. The following subsections detail the modeling and simulation of the Fabry-Pérot interferometer using Lumerical FDTD in an attempt to reproduce the results presented in [198]. Materials considered for the waveguide walls in the simulation of the Fabry-Pérot interferometer discussed later in Section 3.3.5 were real metals, specifically aluminum (Al), silver (Ag), and gold (Au), as well as a PEC. Additionally, the EM source used was a TE-polarized dipole source.

\subsubsection{Dipole Sources and Polarizability}

A dipole moment is defined as the following:

$$
\vec{p}=\delta \vec{x}
$$

Dipoles sources can be classified as either electric or magnetic.

- Electric Dipole - an electric dipole moment, or electric dipole, is a measure of the polarity of a system of electric charges. In (175), $x$ is the displacement vector pointing from the negative charge(s) to the positive charge(s). This fact implies that the electric dipole moment vector also points from the same negative charge(s) to the positive charge(s). 
The $E$-field lines run opposite of the displacement and dipole moment vectors running away from the positive charge(s) to the negative charge(s). Despite these differences, there is no confliction here since the electric dipole moment pertains to charge position, not field lines [236]. Furthermore, an electric dipole can be conceptualized as an oscillating point charge. The polarization, or net electric dipole density $(\vec{P})$, is given by:

$\vec{P}=N \vec{p}$

with the polarization being in units of Coulombs per square meter $\left(\mathrm{C} / \mathrm{m}^{2}\right), N$ is the number of dipoles per unit volume $\left(\mathrm{m}^{-3}\right)$, and $\vec{p}$ is the average electric dipole moment $(\mathrm{C} \cdot \mathrm{m})$ [236]. Presented in Figure 3.5 is the polarization, or net electric dipole moment density.

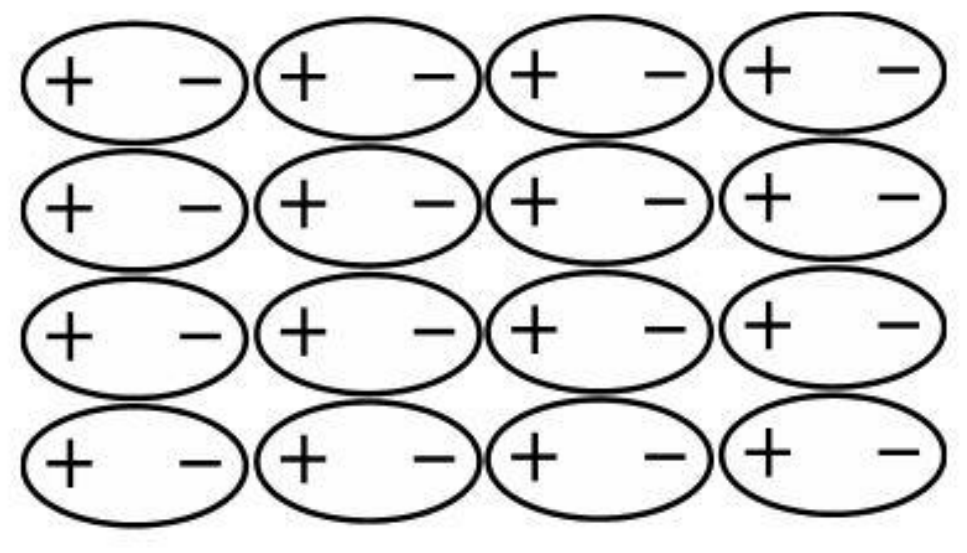

Figure 3.5 Polarization, or net electric dipole density [236].

- Magnetic Dipole - a magnetic dipole moment, or magnetic dipole, originates from a circulating charge. The magnetic dipole moment is a quantity that determines the torque it will experience in the presence of an external $H$-field. A magnetic dipole can be considered to be a vector quantity with a magnitude and direction where the magnetic moment points from its south pole to its north pole. Furthermore, a magnetic dipole can be conceptualized as a current loop. The effect of an applied magnetic field on a magnetic material is to create a net magnetic dipole moment per unit volume. The magnetization or net magnetic dipole moment density is given by:

$\vec{M}=N \vec{m}$ 
with the magnetization being in units of Amperes per meter $(\mathrm{A} / \mathrm{m}), N$ is the number of dipoles per unit volume $\left(\mathrm{m}^{-3}\right)$, and $\vec{m}$ is the average magnetic dipole moment $\left(\mathrm{A} \cdot \mathrm{m}^{2}\right)$ [236]. For some materials, the $H$-field produced is proportional to its magnetic dipole moment per unit volume:

$\bar{M}=\chi_{m} \bar{H}$

where $\bar{M}$ is the induced magnetization, $\chi_{m}$ refers to magnetic susceptibility, and $\bar{H}$ is the magnetic field (both $\bar{M}$ and $\bar{H}$ are in units of $\mathrm{A} / \mathrm{m}$ ) [236]. Presented in Figure 3.6 is the magnetization, or net magnetic dipole moment density.

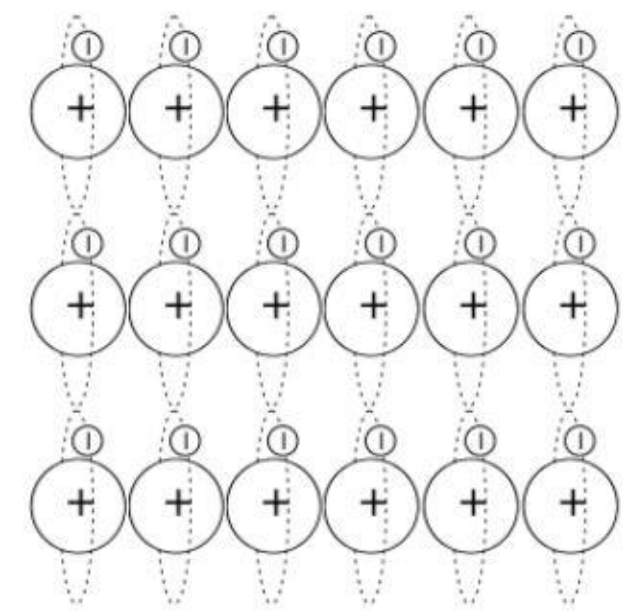

Figure 3.6 Magnetization, or net magnetic dipole moment density [236].

- $\quad$ Polarization Density - also known as electric polarization or just polarization, is the density of permanent or induced electric dipole moments in a dielectric material measured in units of $\mathrm{C} / \mathrm{m}^{2}$ [236]. The density of dipoles is given by:

$\vec{P}=N \vec{p}=N \delta \vec{x}=N \alpha \vec{E}$

The $E$-field polarizes molecules in or around the field itself:

$\vec{P}=\varepsilon_{0} \chi_{e} \vec{E}$ 
where $\chi_{e}$ is the electric susceptibility of a dielectric material - a measure of how easily the electric dipole polarizes in response to an external $E$-field [236]. Polarizability, therefore, provides information on how easily charge distribution can be disturbed like the electron cloud of an atom or molecule from its normal shape by an external $E$-field [236]. Polarizability can be classified into four categories: 1) electronic polarizability $\left(\alpha_{e}\right)-$ polarization of localized electrons, 2) ionic polarizability $\left(\alpha_{i}\right)$ - displacement of ions, 3) dipolar polarizability $\left(\alpha_{d}\right)$ - reorientation of polar molecules, and 4) space charge polarizability $\left(\alpha_{s}\right)$ - long range charge migration [236]. Figure 3.7 contrasts the differences between low and high dipole densities.

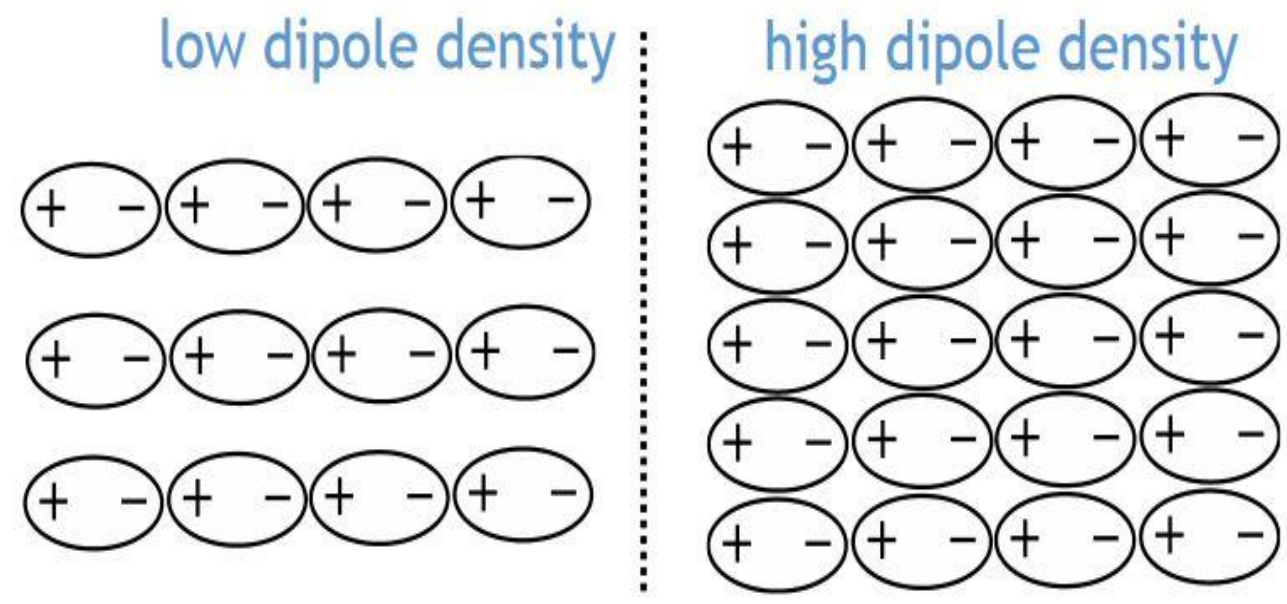

Figure 3.7 Differences between low and high dipole densities [236].

\subsection{TE vs. TM Modes}

The technical definition of polarization conventions will now be discussed. The polarization of incident light upon a grating is defined by the orientation of the $E$ and $H$-fields relative to the plane of incidence. The plane of incidence is defined by the two vectors being the vector of propagation of the illumination and the grating normal [237]. The three conventions used to describe light polarization are transverse electric (TE), transverse magnetic (TM), and unpolarized.

1. TE - polarized light characterized by its $E$-field being perpendicular to the plane of incidence. For TE-polarized light, the $H$-field is always perpendicular to the $E$-field in isotropic materials, and thus lies within the plane of incidence. 
2. TM - polarized light characterized by its $H$-field being perpendicular to the plane of incidence. For TM-polarized light, the $E$-field is always perpendicular to the $H$ field in isotropic materials, and thus lies within the plane of incidence.

3. Unpolarized - unpolarized light equivalent to the average of TE and TM efficiency for the purpose of defining the diffraction efficiency of a grating.

\subsubsection{Skin Depth of Real Metals}

The classical theory of absorption in dielectrics is due to Lorentz and in metals to Drude. Both models treat the optically active electrons in a material as classical oscillators. In the Lorentz model, the electron is considered to be bound to the nucleus by a harmonic restoring force. In this manner, Lorentz's picture is that of the nonconductive dielectric. Drude considered the electrons to be free and set the restoring force in the Lorentz model equal to zero. Both models include a damping term in the electron's equation of motion that, in more modern terms, is recognized as a result of electron-phonon collisions [238]. These models solve for the electron's motion in the presence of the EM field as a driving force. From this, it is possible to write an expression for the polarization induced in the medium and from that to derive the dielectric constant [238]. The Lorentz model for dielectrics gives the relative real and imaginary parts of the dielectric constant $\varepsilon_{1 R}$ and $\varepsilon_{2 R}$ in terms of $N$, the number of dipoles per unit volume, $e$ and $m$, charge and mass of the electron, $\Gamma$, the damping constant, $\omega$ and $\omega_{0}$, the radian frequencies of the field and the harmonically bound electron, and $\varepsilon_{0}$, the permittivity of free space [238].

The range of frequencies where $\varepsilon_{1}$ increases with frequency is referred to as the range of normal dispersion, and the region near where $\omega=\omega_{0}$, it decreases with frequency is called the range of anomalous dispersion. Since the ionic polarizability is much smaller than the electronic polarizability at optical frequencies, only the electronic terms are considered when evaluating optical absorption using the Lorentz model for dielectrics. The Drude model for metals assumes that the electrons are free to move. This effectively means the Drude model is identical to the Lorentz model, except that is $\omega_{0}$ equals zero in the Drude model [238]. The real and imaginary parts of the dielectric constant are given by:

$$
\varepsilon_{1 R}=1-\left(N e^{2} \varepsilon_{0} m\right) \frac{1}{\omega^{2}+\Gamma^{2}}
$$




$$
\varepsilon_{2 R}=\left(N e^{2} \varepsilon_{0} m\right) \frac{\Gamma}{\omega\left(\omega^{2}+\Gamma^{2}\right)}
$$

The quantity $\Gamma$ is related to the mean time between electron collisions with lattice vibrations, and by considering electronic motion in an $E$-field possessing a radian frequency $(\omega)$, an expression for the average velocity can be obtained. An expression for the conductivity $(\sigma)$ and the parts of the dielectric constant can then be restated. At low EM frequencies, it can be shown that $\varepsilon_{2} \gg \varepsilon_{1}$, and therefore follows:

$$
\alpha=\left(\frac{\omega \mu \sigma}{2}\right)^{1 / 2}
$$

Based on (183), optical properties and the conductivity of a metal are related by the motion of free electrons [238]. At high frequencies, transitions involving conduction or valence band electrons are possible that will cause a noticeable deviation from the Drude model; however, experimental data reported for most metals are in congruence with Drude predictions at wavelengths as short as $1 \mu \mathrm{m}$ [238]. Taking (183) further, it is clearly observable that an EM field propagating in a metal will attenuate by a factor of 1/e when it has traveled a distance:

$$
\delta=(2 / \omega \mu \sigma)^{1 / 2}
$$

The above quantity is known as skin depth, or the skin effect, and is approximately $50 \mathrm{~nm}$ for most metals at optical frequencies. Additionally, after light has propagated one skin depth into a metal, its intensity reduces to $1 / e$ of its value at the surface of the metal [238].

\subsubsection{Poynting Vector}

EM waves possess energy densities associated with the $E$ - and $H$-fields as the waves pass through space [239]. Per unit area, the rate of transport, known as the Poynting vector, is defined as:

$$
\vec{S}=\frac{1}{\mu_{0}} \vec{E} \times \vec{B}
$$


Since the $H$-field is perpendicular to the $E$-field, the magnitude of the Poynting vector can be modified:

$$
S=\frac{1}{\mu_{0}} E B
$$

$S$ is perpendicular to both $E$ and $B$ following the direction of propagation of the wave [239]. A solution to the plane wave equation given $B_{\mathrm{m}}=E_{\mathrm{m}} / c$ is:

$$
S=\frac{1}{c \mu_{0}} E_{m}^{2} \overline{\sin ^{2}(k x-\omega t)}=\frac{1}{c \mu_{0}} \frac{E_{m}^{2}}{2}
$$

(187) above relies on the fact that the average of the square of a sinusoidal function over a whole number of periods is equal to $1 / 2$ [239].

\subsubsection{Fabry-Pérot Cavity Resonance Comparisons}

A Fabry-Pérot cavity mode was modeled and simulated using FDTD to determine cavity resonance and power transmission through the cavity itself. The cavity dimensions as shown in Figure 3.8 were as follows: four PEC plates 65-nm thick and 1,000-nm long, $d=500 \mathrm{~nm}$, and gap widths of $100 \mathrm{~nm}$. PEC plates were initially used in modeling and determining resonance, however real metal plates such as aluminum $(\mathrm{Al})$, gold $(\mathrm{Au})$, and silver $(\mathrm{Ag})$ were also investigated for comparison (Figure 3.9). Figure 3.10 shows power recorded as a function of monitor distance from the cavity opening opposite of the dipole source.

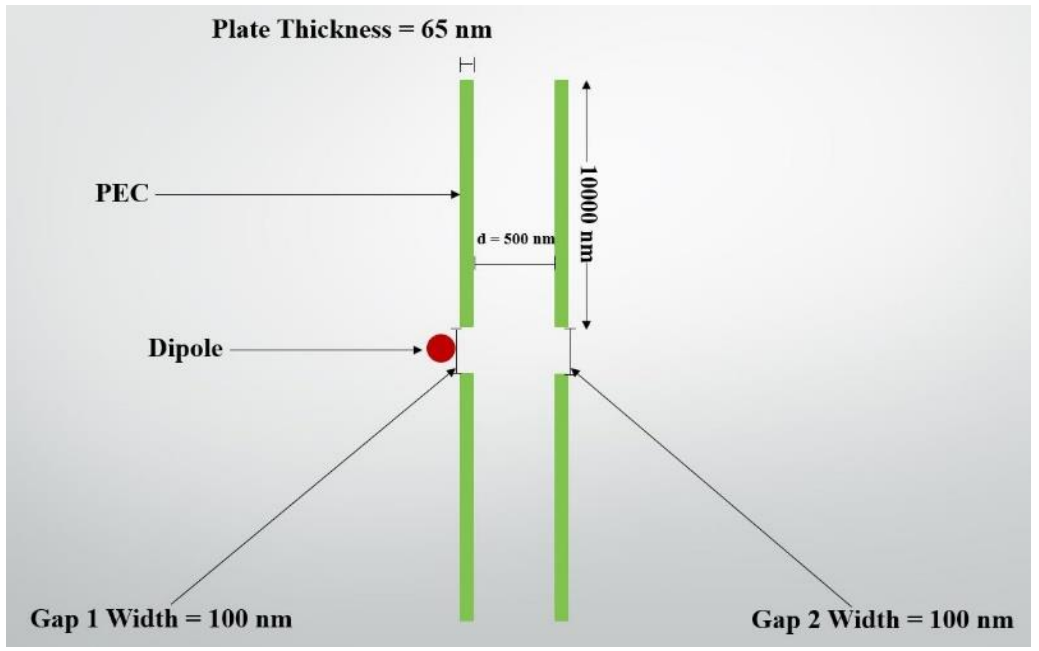

Figure 3.8 Cavity geometry. Fabry-Pérot interferometer simulation layout with dimensions similar to the cavity geometry presented in [198]. 


\subsubsection{Cavity Mode Simulation Results}

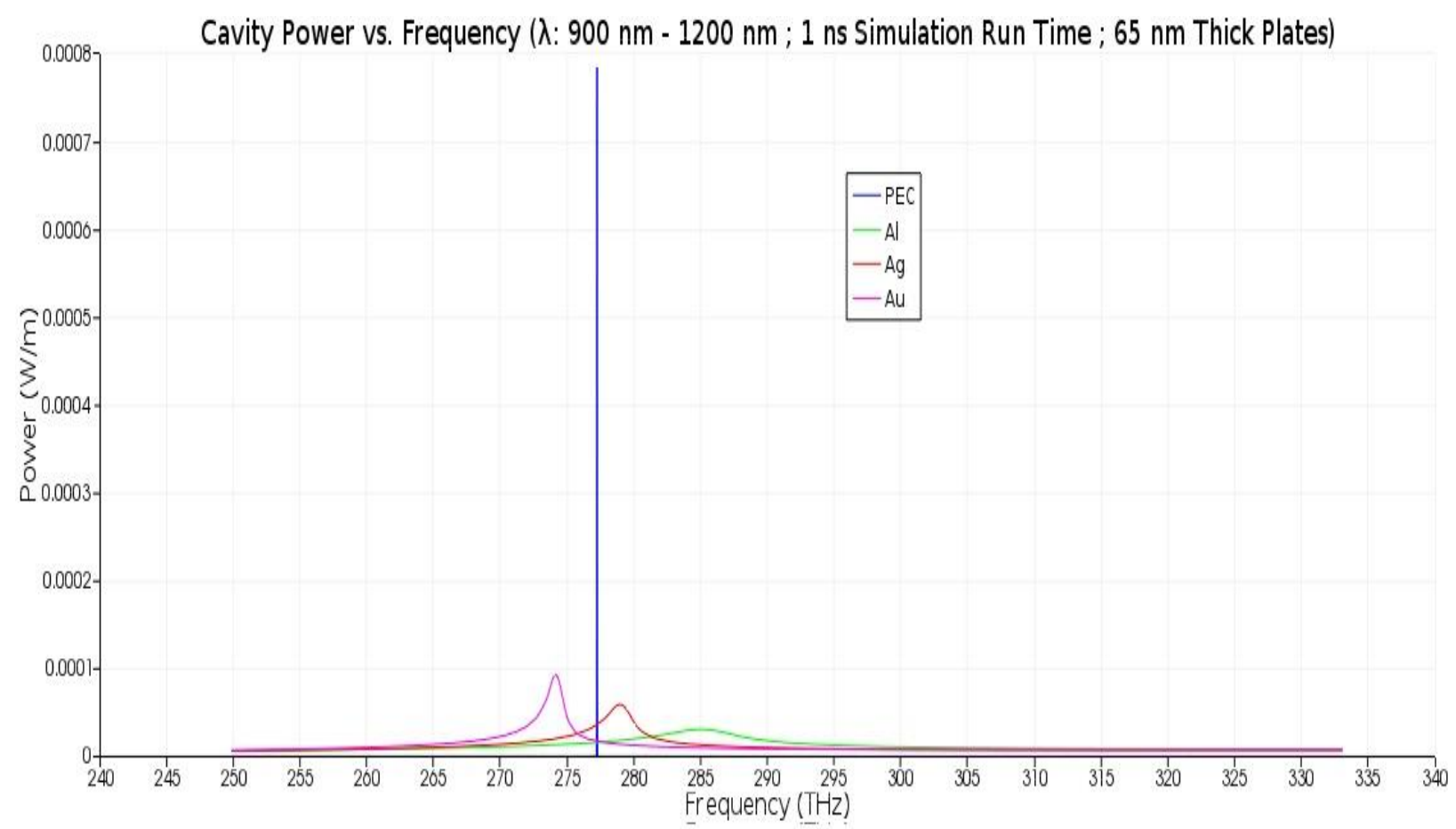

Figure 3.9 Resonance comparison of the Fabry-Pérot cavity mode for $\mathrm{Al}, \mathrm{Ag}, \mathrm{Au}$, and PEC plates.

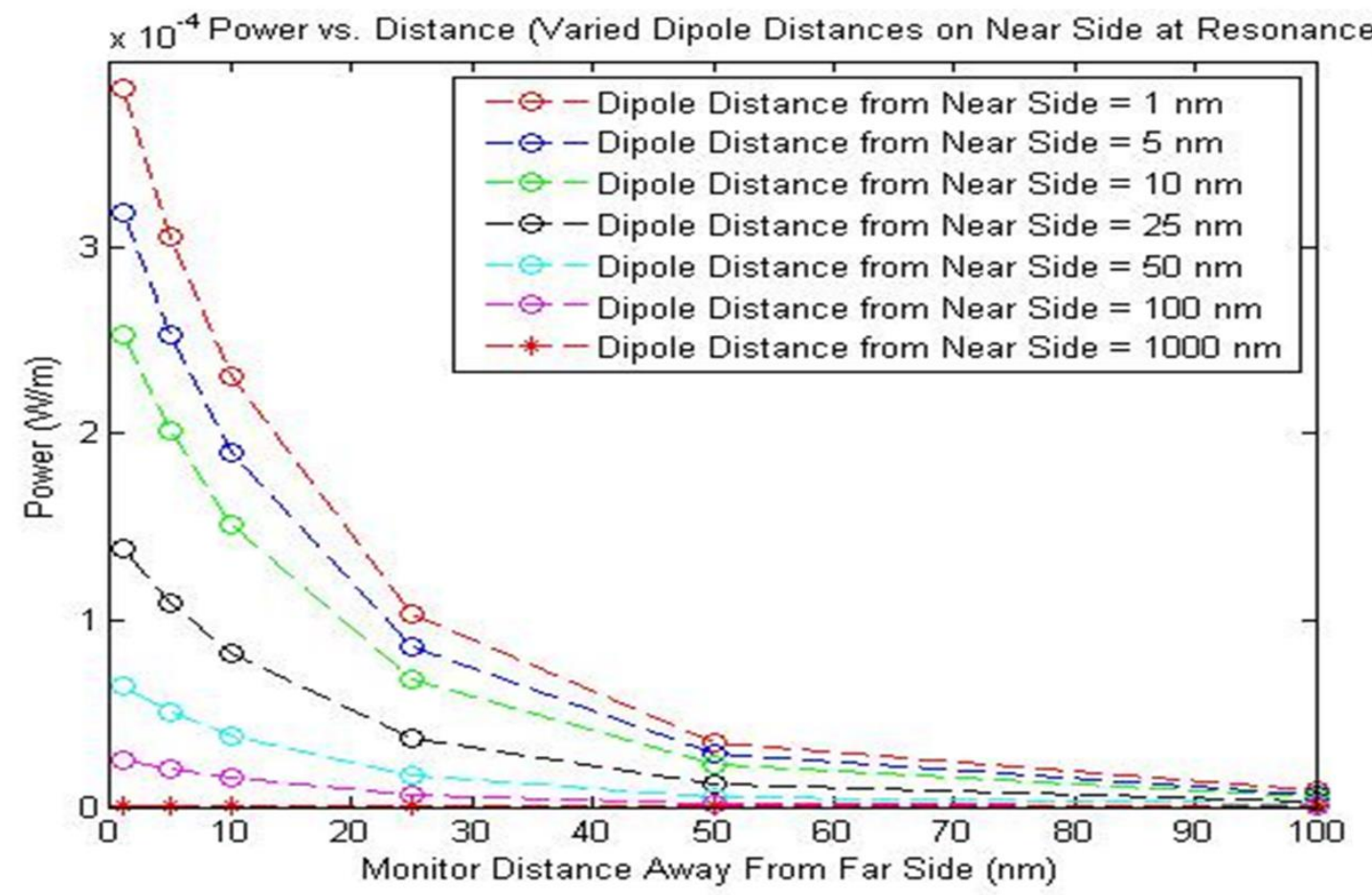

Figure 3.10 Recorded power on far side of cavity (opposite of the dipole source) as a function of dipole distance from the near side of the cavity. 


\subsection{Plasmonic Lattices of Arrays of Gold Nanoparticles}

The purpose of the various parametric analyses conducted initially in this thesis was to find a set of lattice parameters that will optimize the emission enhancement of cadmium selenide/zinc sulfide $(\mathrm{CdSe} / \mathrm{ZnS})$ QDs suspended in toluene possessing an emission spectrum centered at approximately $565 \mathrm{~nm}$. Specifically, this optimization effort began with the exploitation of surface plasmons across a periodic plasmonic lattice of square pillar geometries to optimize the excitation and, ideally, the emission rate enhancement of an ensemble of fluorophores for LOC fluorescence biosensing as presented in [187]. Although these simulations were engineered for enhancing the excitation rate of $\mathrm{CdSe} / \mathrm{ZnS}$ QDs suspended in toluene, these optimized plasmonic lattice parameters are applicable to other commercially available QDs and fluorophores possessing emission spectra that overlap with the resonance condition of the lattice.

\subsubsection{Parametric Sweeps of Critical Lattice Parameters}

The design layout of the original periodic structure constructed in the simulation environment based on [187] can be seen in Figure 3.11 with the initial composition and dimensionality provided in Figure 3.12. Contained in Section 4.1.1 to Section 4.1.4 are the initial $E$-field enhancements found square, cylindrical, equilateral star, and equilateral triangle nanopillar geometries. Detailed here is the methodology behind the initial parameter optimization effort for a plasmonic lattice comprised of arrays of square nanopillars as presented in [187].

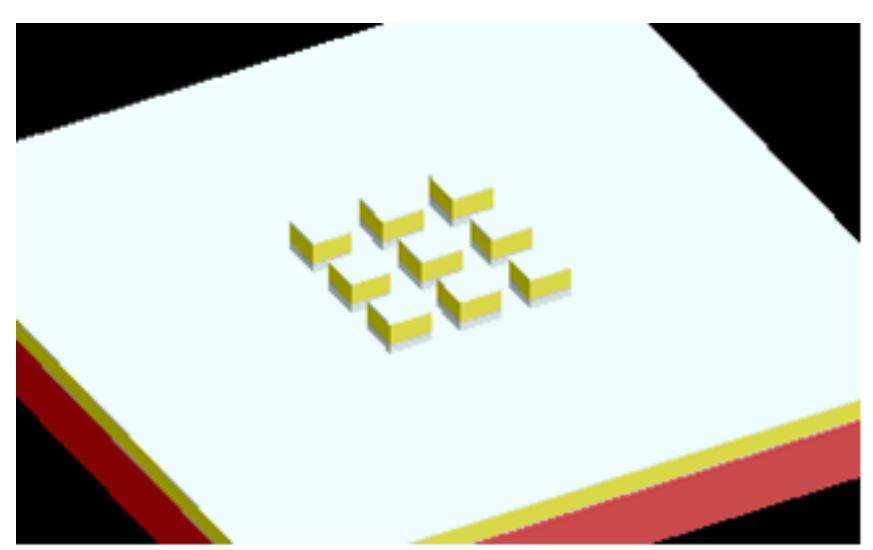

Figure 3.11 3D Perspective Simulation View of Initial Square Lattice Comprised of Square Pillars. 


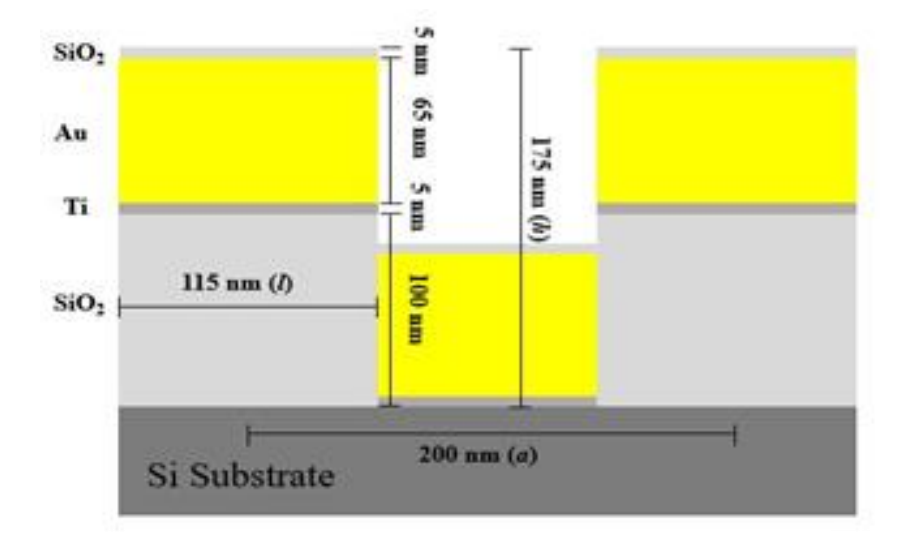

Figure 3.12 Cross-Sectional View of Initial Pillar Composition and Dimensions.

The QDs were modeled in the software as a pulsed plane wave source for determining the resonance of the plasmonic lattice. A 200-nm thick silicon ( $\mathrm{Si}$ ) base was used as the substrate upon which the periodic plasmonic lattice was built. A 3x3 array of square silicon dioxide $\left(\mathrm{SiO}_{2}\right)$ nanopillars was created atop the Si substrate each with the following dimensions: $115 \mathrm{~nm}$ in length $(l), 100 \mathrm{~nm}$ in height $(h)$, and a lattice constant (a) of $200 \mathrm{~nm}$. A 5-nm thick layer of titanium (Ti) was added over the entire substrate and each pillar to serve as the adhesive layer between the $\mathrm{SiO}_{2}$ and Au. A 65-nm thick Au layer was then layered atop the Ti-covered substrate and pillars. Finally, a 5-nm thick $\mathrm{SiO}_{2}$ spacer layer was inserted atop the lattice structure to reduce the effects of quenching. The boundary conditions of the simulation were configured as follows: periodic in the $x$ and $y$-directions, and perfectly matched layer (PML) in the $z$-directions.

Periodic boundary conditions are utilized when both the physical structures and EM fields are repeated throughout the system, allowing the user to accurately observe the entire response of the system by simulating a single unit cell [192]. In the software tool, the PML forces the boundary conditions to match the impedance of the adjacent materials to prevent reflection while also absorbing any incident light; essentially simulating an open boundary [192]. The refractive index (n) of the simulation region was set to 1.497 corresponding to the $n$ of the toluene solution in which the QDs are suspended. The simulation run time was set to 1,000 fs to allow for enough optical cycles respective to the pulse length of the plane wave source injected into the simulation region. For mesh refinement, the 'conformal variant 1' option was selected because of the Au layers present in the plasmonic device design. The parameters investigated for the square pillars comprising the array were as follows: $a, l$, and $h$. The first parameter $a$ directly impacts the other geometric parameters and fabrication options (i.e., $a$ restricts $l$, and $h$ ). 
This parametric analysis was prefaced with an original set of features corresponding to the emission $\lambda$ of the $\mathrm{CdSe} / \mathrm{ZnS}$ QDs as previously discussed in [187]. More explicitly, prior to carrying out the parametric sweep of $a, l$ was kept at $115 \mathrm{~nm}, h$ was set to $170 \mathrm{~nm}$, comprised of a 100-nm thick $\mathrm{SiO}_{2}$ layer, 5-nm thick Ti layer, and a 65-nm thick Au layer, with a pulsed plane wave source ranging from $300 \mathrm{~nm}$ to $800 \mathrm{~nm}$ positioned $1 \mu \mathrm{m}$ above the array. A point monitor was placed at the surface of the center pillar within the $3 \times 3$ array configured to record the $E$-field at that location. 


\section{Chapter 4}

\section{Simulation Results}

Chapter 4 contains all of the simulation results obtained from both the initial parametric optimizations and the final parametric optimizations. The initial lattice parametric sweeps were based on those presented in [187] where the optimization began with a given parameter set with the final optimization effort evaluating a much larger parameter space by treating each critical lattice parameter independently. 


\subsection{Initial Critical Lattice Parameter Optimizations}

\subsubsection{Square Nanopillars}

The $E$-field data obtained from this point monitor were compared at each $a$ ranging from $150 \mathrm{~nm}$ to $400 \mathrm{~nm}$ in 5-nm increments. The data was recorded and analyzed to observe which $a$ yielded the greatest $E$-field enhancement at the surface of the structure given the initial parameters from [187]. Figure 4.1 shows the $E$ vs. $a$ vs. $\lambda$ data obtained used for the comparison of the $E$-field enhancement for determining the optimal value of $a$ ( $\left.a_{\text {optimal }}\right)$ that yielded the greatest $E$-field enhancement at the structure surface. Upon analysis of the data, it was found that $a_{\text {optimal was }} 270$ $\mathrm{nm}$ for the square nanopillars.

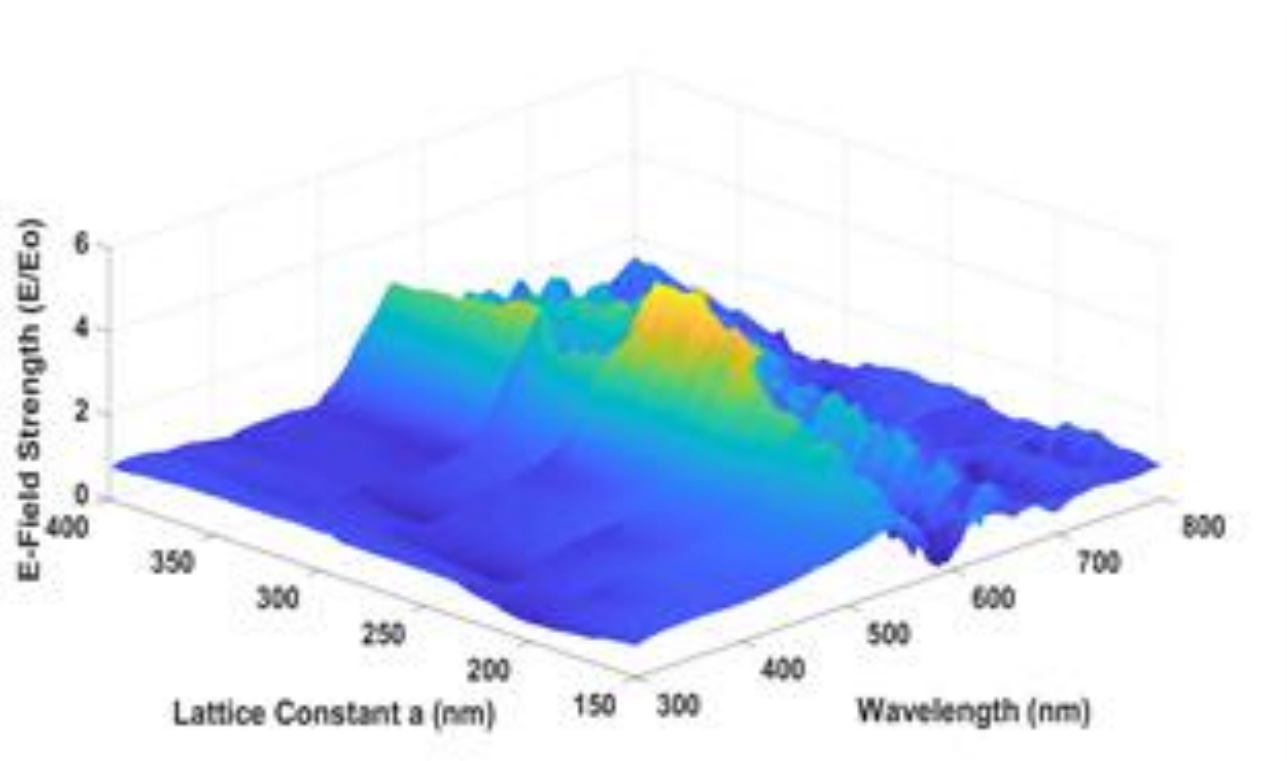

Figure 4.1 $E$ vs. $a$ vs. $\lambda$ parametric sweep data obtained from the point monitor showing peak $E$-Field occurred at $a=270 \mathrm{~nm}$.

After optimizing $a, l$ was varied from $50 \mathrm{~nm}$ to $215 \mathrm{~nm}$ in 5 -nm increments with $a=270$ nm. Figure 4.2 shows the $E$ vs. $l$ vs. $\lambda$ data from which the optimal value of $l\left(l_{\text {optimal }}\right)$ was determined. Here, the point monitor data obtained from the parametric sweep of $l$ delineated that the greatest $E$-field enhancement occurred at $l=50 \mathrm{~nm}$. 


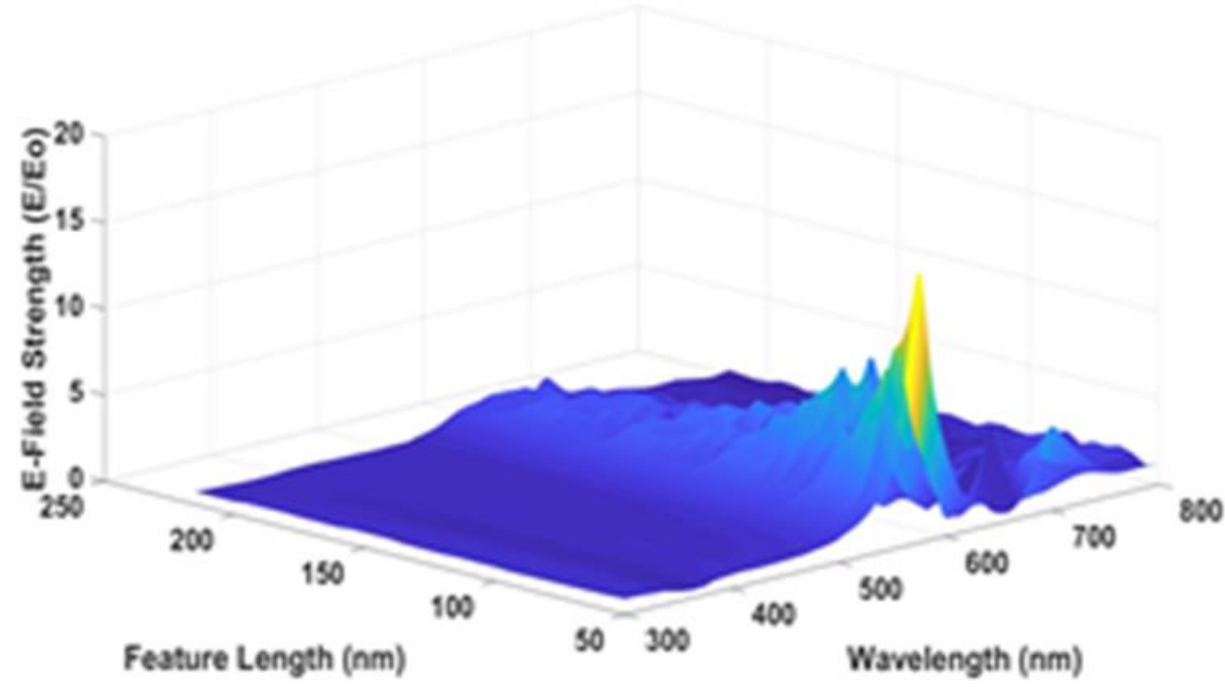

Figure 4.2 $E$ vs. $l$ vs. $\lambda$ parametric sweep data obtained from the point monitor showing peak $E$-Field occurred at $l=50 \mathrm{~nm}$.

Next, $h$ was parametrically swept from $115 \mathrm{~nm}$ to $210 \mathrm{~nm}$ in 5-nm increments incorporating $a_{\text {optimal }}$ and $l_{\text {optimal }}$ into the sweep. Note that $h$ was varied by sweeping the height, or thickness $(t)$, of the Au layer atop the square nanopillars. This was done because of the nature of the fabrication process where the $\mathrm{SiO}_{2}$ layer and the Ti adhesion layer remained constant at $h=100 \mathrm{~nm}$ and $h=$ $5 \mathrm{~nm}$, respectively. Figure 4.3 shows the $E$ vs. $h$ vs. $\lambda$ data from which the optimal value of $h$ ( $\left.h_{\text {optimal }}\right)$ was determined. The data showed that the greatest $E$-field enhancement occurred at $h=$ $175 \mathrm{~nm}$, or a 70-nm thick Au layer. 


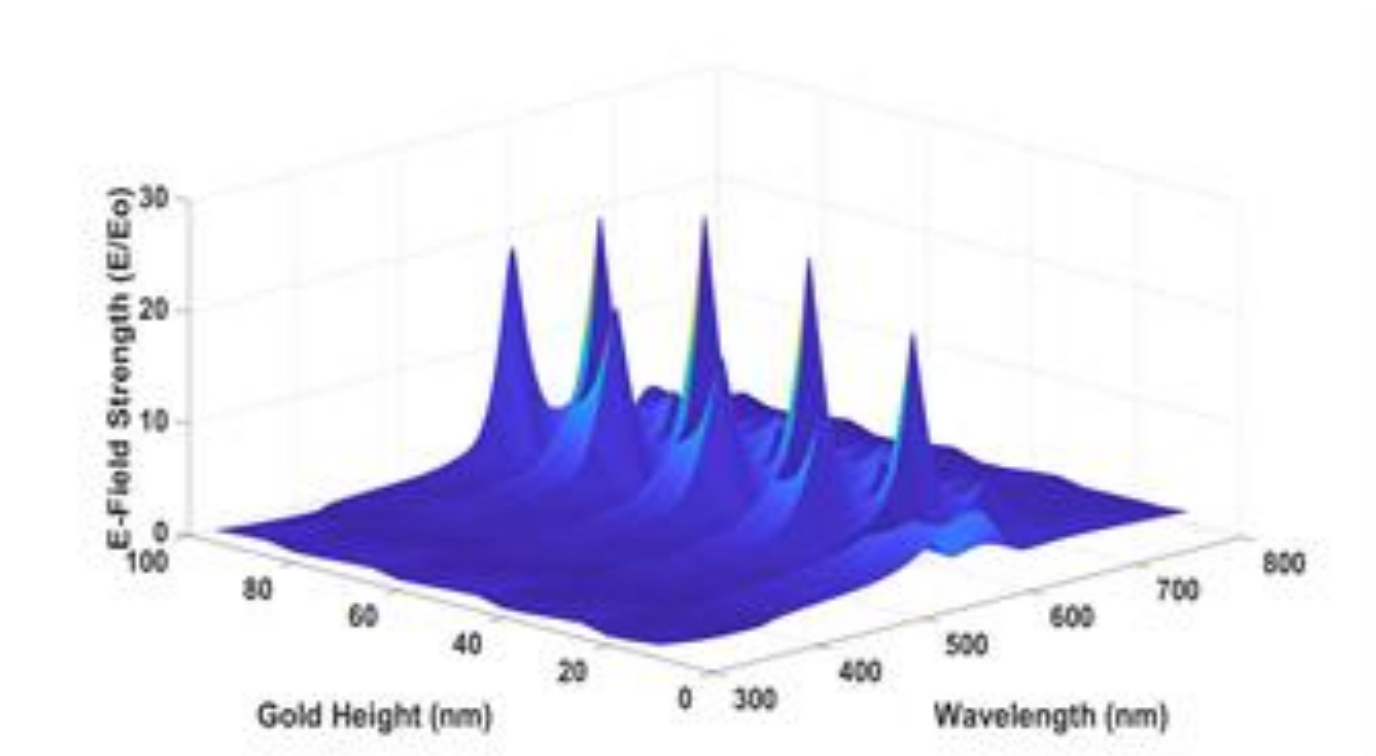

Figure 4.3 $E$ vs. $h$ vs. $\lambda$ parametric sweep data obtained from the point monitor. The data shows the peak $E$-Field occurred at $h=175$ nm (70-nm thick Au Layer)

Upon completion of the parametric optimizations, a final simulation was performed with each of the optimized parameters incorporated into the device design. Like each of the previous parametric sweeps, the plane wave source pulse ranged from $300 \mathrm{~nm}$ to $800 \mathrm{~nm}$ to determine resonance. Data analysis of the optimized plasmonic lattice structure indicated that the peak $E$ field at the structure surface occurred where $\lambda=596.593 \mathrm{~nm}$. While not at the peak emission $\lambda$ of the CdSe/ZnS QDs, this $\lambda$ does lie within their emission spectrum ( 500 nm - 610 nm) [187]. Finally, the same simulation was repeated with a CW plane wave emitting at $\lambda=596.593 \mathrm{~nm}$. Figure 4.4 shows a top-down view of the plasmonic lattice excited at this $\lambda$. Multiple peaks within the $500 \mathrm{~nm}$ to $600 \mathrm{~nm}$ range of the visible regime were observed throughout each sweep for each geometry, consistent with the Au NP plasmonic range. Also visualized in Figure 4.4 is the optimized lattice structure exposed to a plane wave source at $\lambda=565 \mathrm{~nm}$ for comparison against the results obtained from the initial structure design in [187]. Table 4.1 catalogues each of the optimal lattice parameters found for the square nanopillar geometry. 

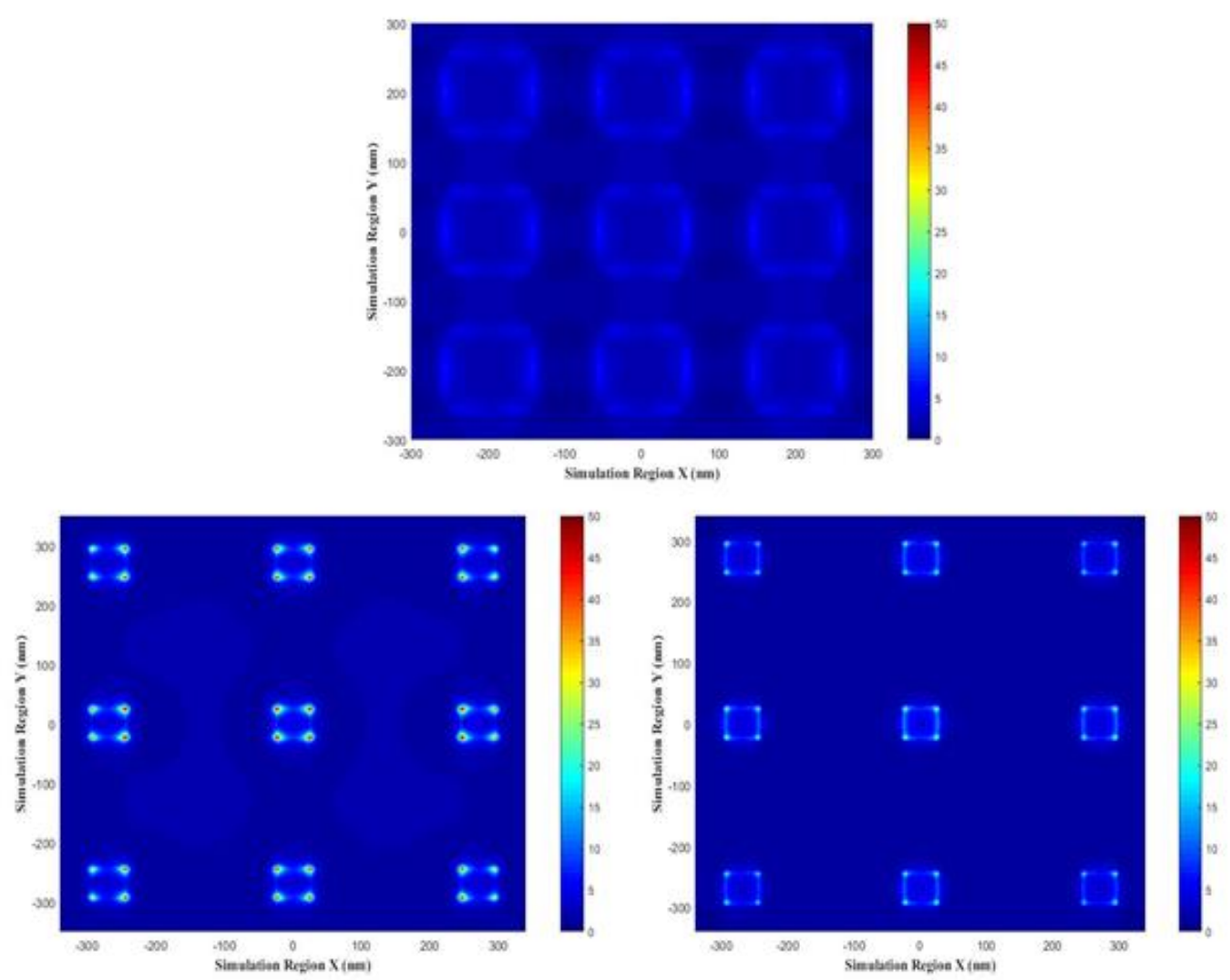

Figure 4.4 (Top Center) surface-enhanced $E$-field enhancement $\left(|E| /\left|\mathrm{E}_{0}\right|\right)$ of 6 reproduced from [240] with a plane wave source emitting at $\lambda=565$ $\mathrm{nm}$, (Bottom Left) surface-enhanced $E$-field enhancement $\left(|\mathrm{E}| / \mathrm{E}_{0} \mid\right)$ of 61 of the parametrically optimized lattice with a plane wave source emitting at $\lambda=596.593 \mathrm{~nm}$, and (Bottom Right), surface-enhanced $E$-field enhancement $\left(|\mathrm{E}| / \mathrm{E}_{0} \mid\right)$ of 30 of the parametrically optimized lattice with a plane wave source emitting at $\lambda=565 \mathrm{~nm}$. The same dynamic range of 0 (dark blue) to 50 (red) was used for direct comparison.

Table 4.1 Parameter ranges swept for the square nanopillar geometry.

\begin{tabular}{|c|c|c|}
\hline Parameter & Sweep Range (nm) & Optimal (nm) \\
\hline$a$ & $150-400$ & 270 \\
\hline$l$ & $50-215$ & 50 \\
\hline$h$ & $115-210$ & 175 \\
\hline
\end{tabular}

As visualized in Figure 4.4, the optimization effort based on the nanostructure dimensions presented produced $E$-field enhancements over an entire order of magnitude than that reported in [240] (61 at $\lambda=596.593 \mathrm{~nm}$ and 30 at $\lambda=565 \mathrm{~nm}$ from this optimization versus $\sim 4$ at $\lambda=565 \mathrm{~nm}$ ). The subsequent parametric analyses performed followed a very similar approach to that of the square pillars except the feature size sweeps began at $l=50 \mathrm{~nm}$ (triangular pillars), $r=25 \mathrm{~nm}$ (cylinders), and $l=50 \mathrm{~nm}$ (nanostars) based on the square $l_{\text {optimal }}$. 
Basing the feature length and radii sweeps on the results obtained from the square pillar parameter sweeps allowed for a greater range in the sweep of $a$. Additionally, the same square lattice configuration pictured in Figure 3.11 was used for the other three feature geometries analyzed. The following subsections provide the contour plots for the other three aforementioned pillar geometries showing the parameter sweeps as well as the top-down and cross-sectional $E$-field intensity plots incorporating the optimal parameters observed.

\subsubsection{Cylindrical Nanopillars}

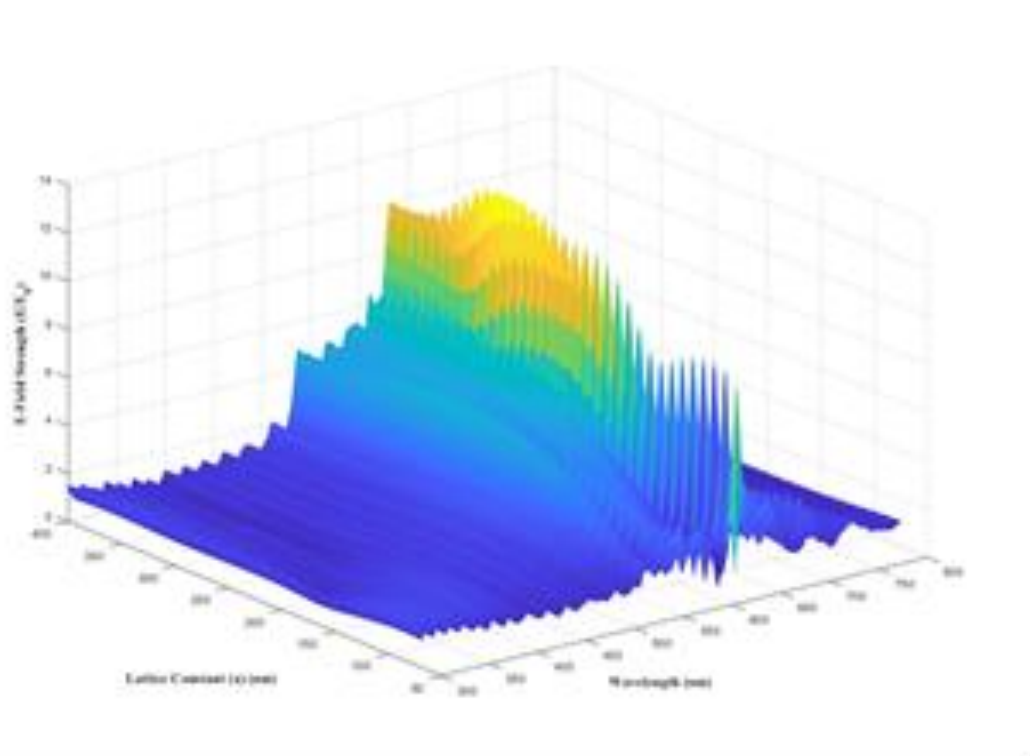

Figure 4.5 $E$ vs. $a$ vs. $\lambda$ parametric sweep data obtained from the point monitor. The data shows the peak $E$-field occurred at $a=275 \mathrm{~nm}$.

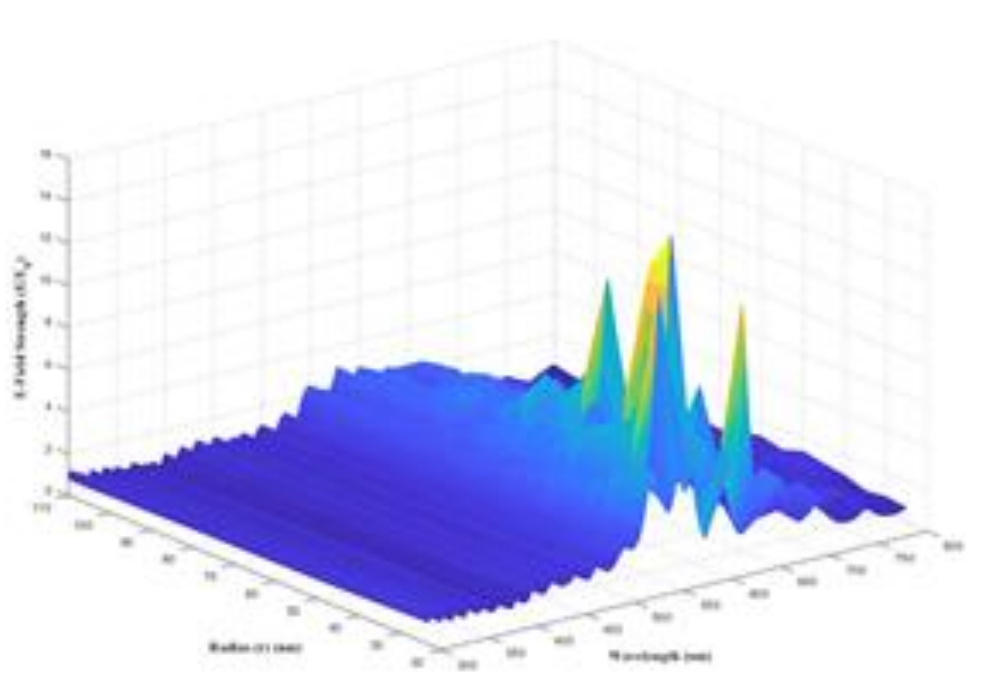

Figure 4.6 $E$ vs. $r$ vs. $\lambda$ parametric sweep data obtained from the point monitor. The data shows the peak $E$-field occurred at $r=35 \mathrm{~nm}$. 


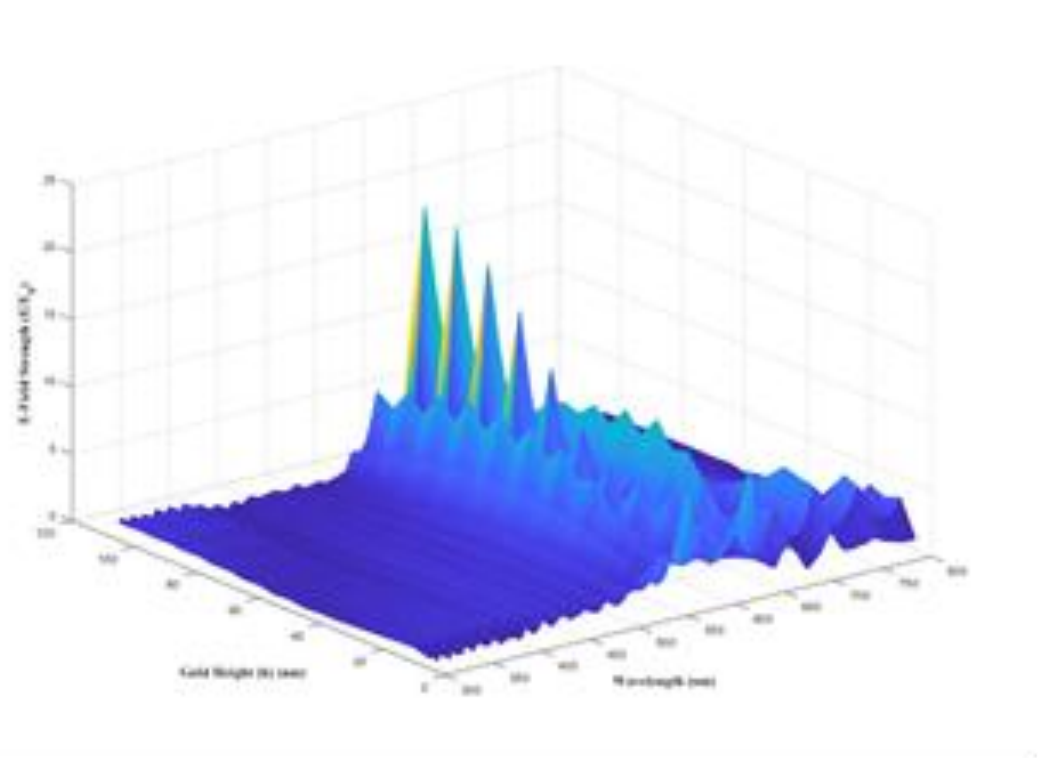

Figure 4.7 $E$ vs. $h$ vs. $\lambda$ parametric sweep data obtained from the point monitor. The data shows the peak $E$-Field occurred at $h=205 \mathrm{~nm}(100-$ nm thick Au Layer)

Table 4.2 summarizes the optimal quantities observed for each of the critical parameters for the cylindrical pillars with Figure 4.8 delineating the simulated lattice of cylindrical nanopillars at resonance incorporating the optimal parameters.

Table 4.2 Parameter ranges swept for the cylinder nanopillar geometry.

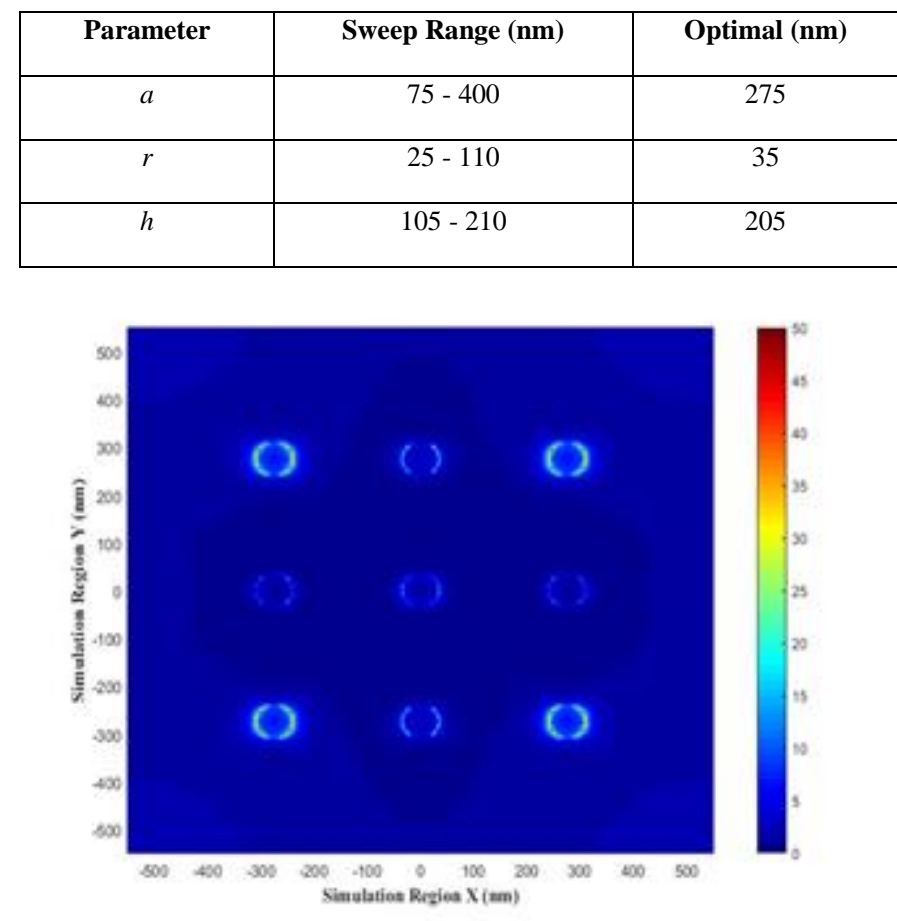

Figure 4.8 Surface-enhanced $E$-field $\left(|\mathrm{E}| /\left|\mathrm{E}_{0}\right|\right)$ of the parametrically optimized lattice with a plane wave source emitting at $\lambda=599.7 \mathrm{~nm}$. 


\subsubsection{Equilateral Star Nanopillars}

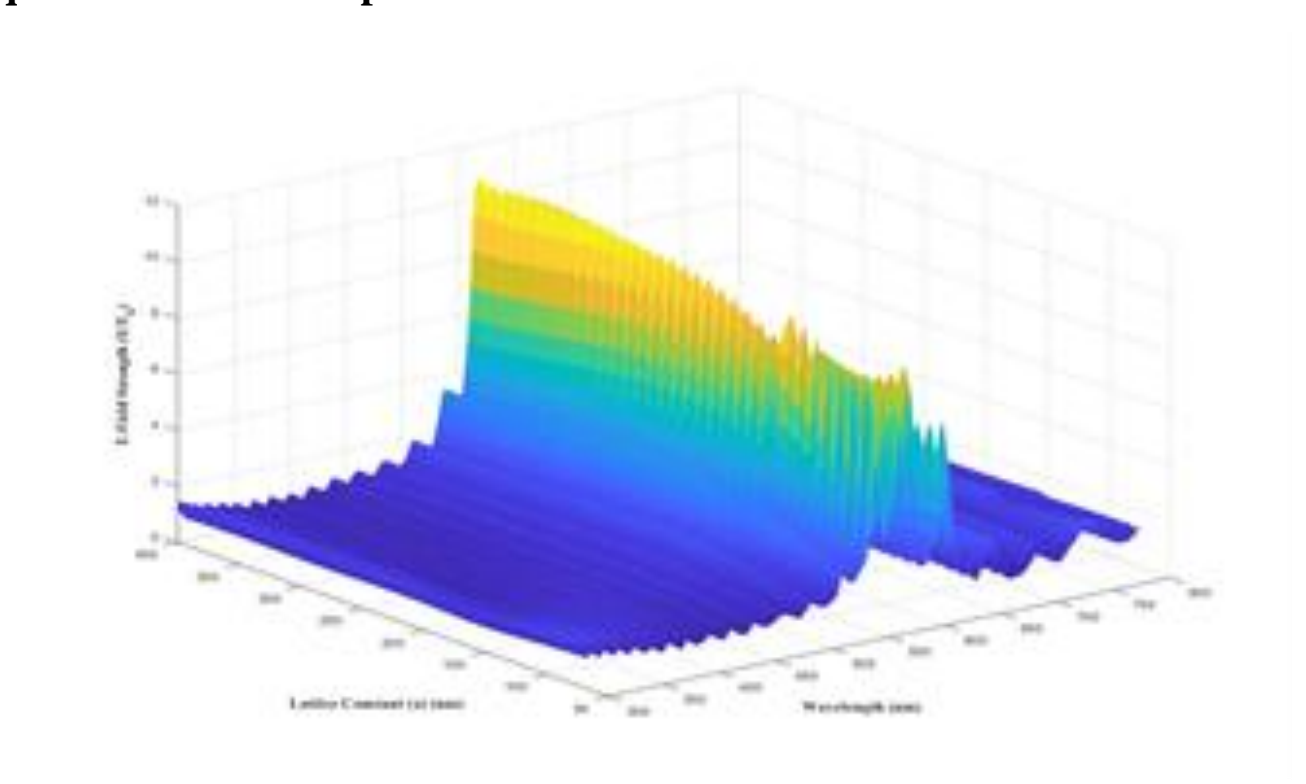

Figure 4.9 $E$ vs. $a$ vs. $\lambda$ parametric sweep data obtained from the point monitor. The data shows the peak $E$-field occurred at $a=400$ nm.

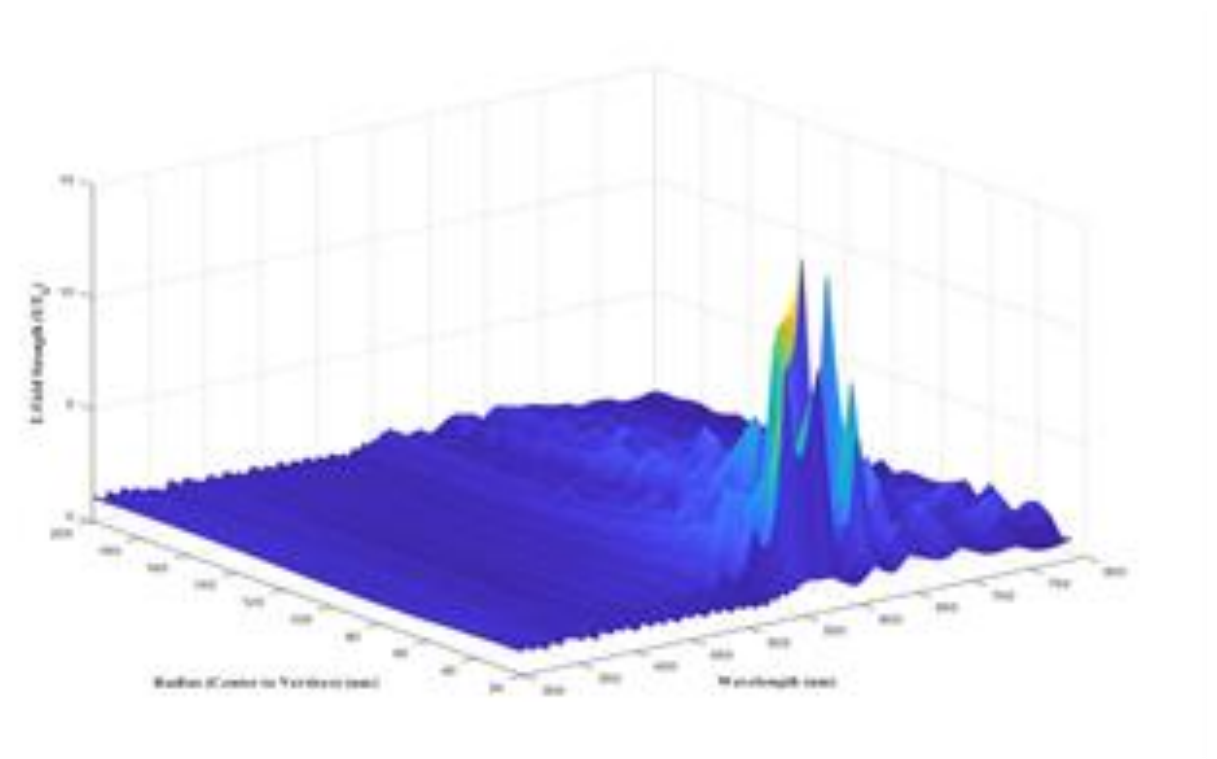

Figure 4.10 $E$ vs. $r$ vs. $\lambda$ parametric sweep data obtained from the point monitor. The data shows the peak $E$-Field occurred at $r_{\text {center-to-vertices }}=45$ $\mathrm{nm}$, rinner-points $=22.5 \mathrm{~nm}$. 


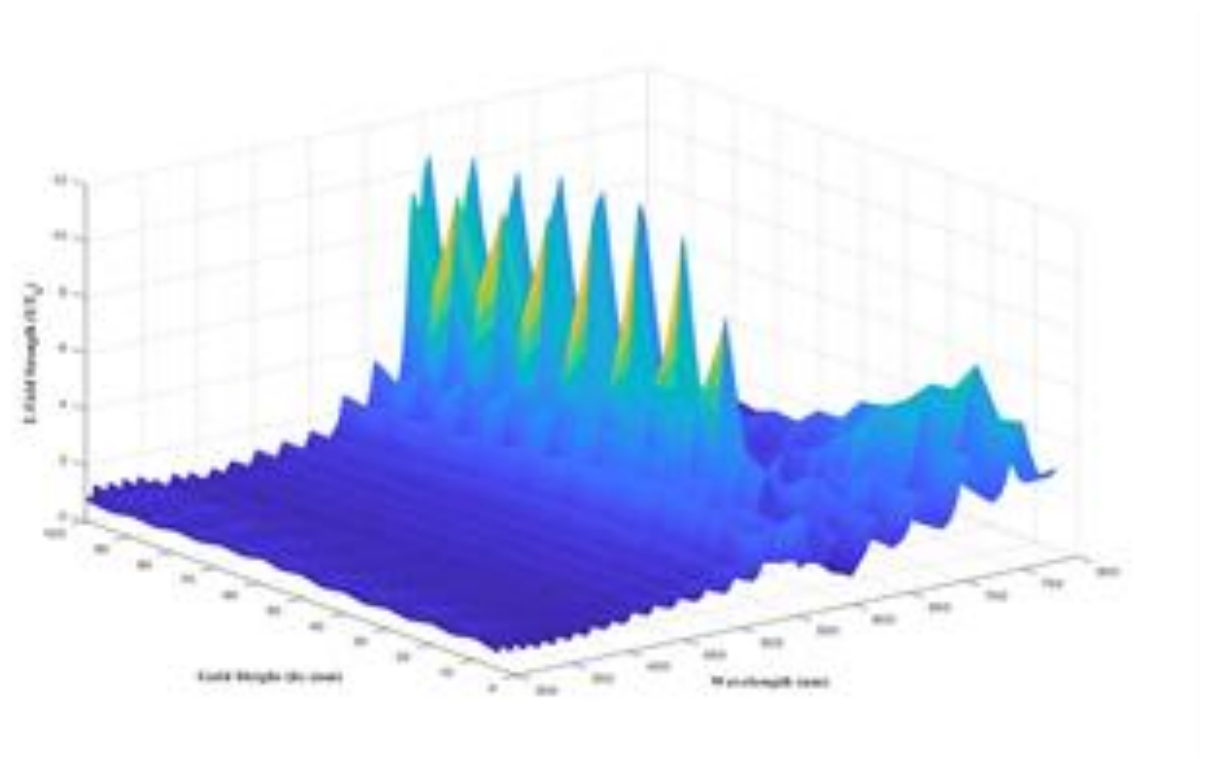

Figure 4.11 $E$ vs. $h$ vs. $\lambda$ parametric sweep data obtained from the point monitor. The data shows the peak $E$-field occurred at $h=155 \mathrm{~nm}(50-\mathrm{nm}$ thick Au Layer).

Table 4.3 summarizes the optimal quantities observed for each of the critical parameters for the equilateral nanostar pillars with the simulated lattice of equilateral nanostar nanopillars at resonance incorporating the optimal parameters shown in Figure 4.12.

Table 4.3 Parameter ranges swept for the equilateral star nanopillar geometry.

\begin{tabular}{|c|c|c|}
\hline Parameter & Sweep Range (nm) & Optimal (nm) \\
\hline$a$ & $75-400$ & 400 \\
\hline$l$ & $25-100$ & 90 \\
\hline$h$ & $105-205$ & 155 \\
\hline
\end{tabular}




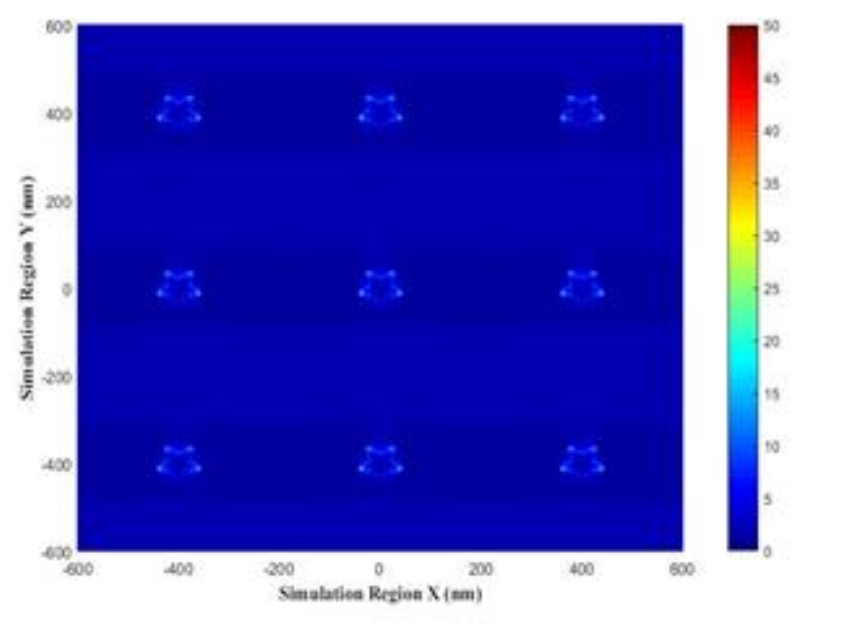

Figure 4.12 Surface-enhanced $E$-field $\left(|\mathrm{E}| /\left|\mathrm{E}_{0}\right|\right)$ of the parametrically optimized lattice with a plane wave source emitting at $\lambda=601.205 \mathrm{~nm}$.

\subsubsection{Equilateral Triangular Nanopillars}

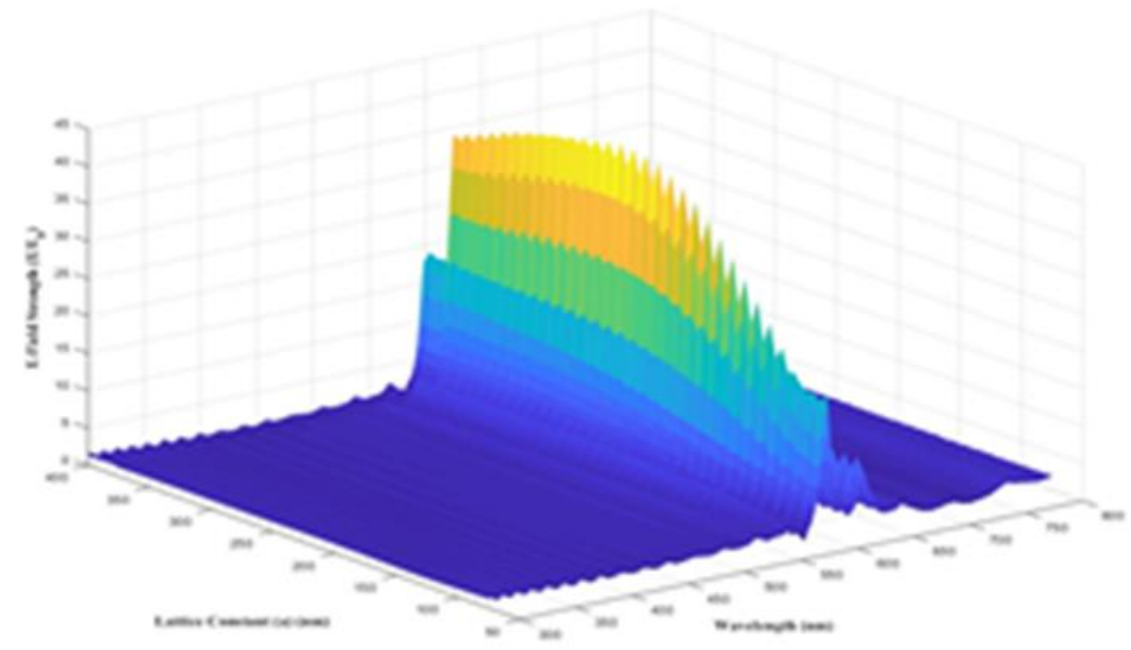

Figure 4.13 $E$ vs. $a$ vs. $\lambda$ parametric sweep data obtained from the point monitor. The data shows the peak $E$-field occurred at $a=265 \mathrm{~nm}$. 


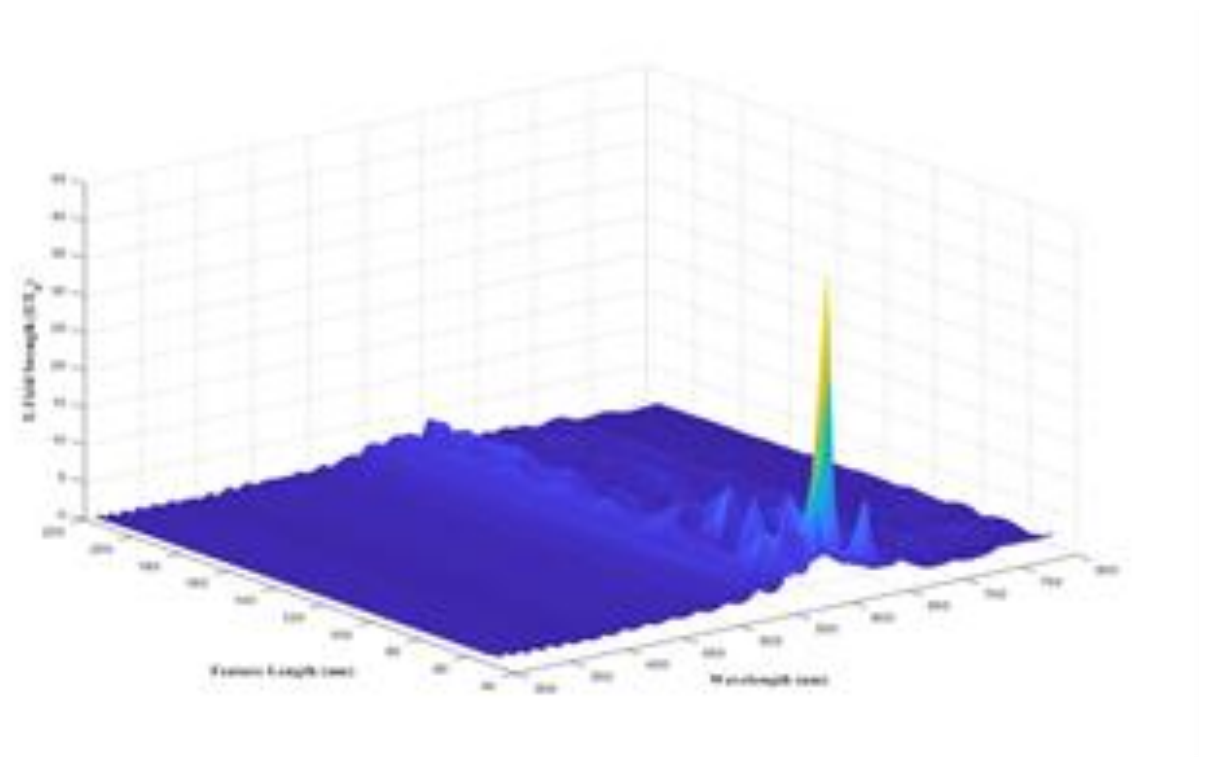

Figure 4.14 $E$ vs. $l$ vs. $\lambda$ parametric sweep data obtained from the point monitor. The data shows the peak $E$-field occurred at $l=50 \mathrm{~nm}$.

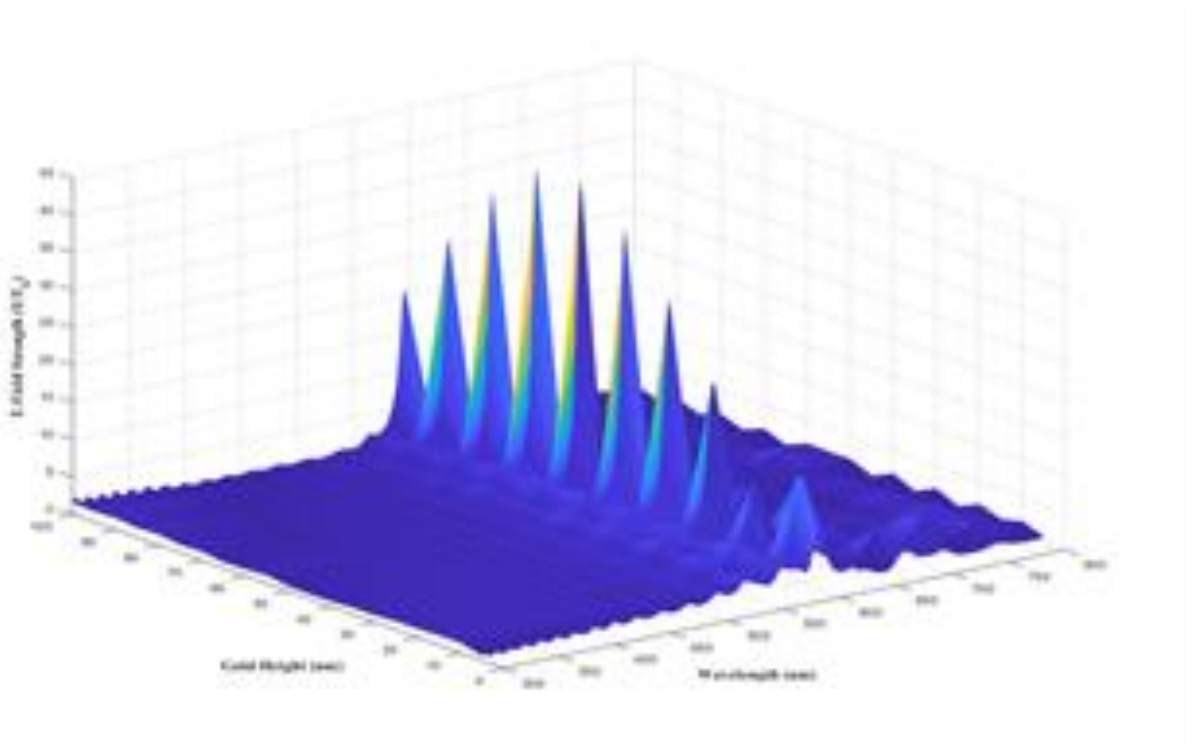

Figure 4.15 $E$ vs. $h$ vs. $\lambda$ parametric sweep data obtained from the point monitor. The data shows the peak $E$-Field occurred at $h=165 \mathrm{~nm}(60-$ nm thick Au Layer).

Table 4.4 summarizes the optimal quantities observed for each of the critical parameters for the equilateral triangular pillars with the simulated lattice of the equilateral triangular nanopillars at resonance incorporating the optimal parameters shown in Figure 4.16. Table 4.5 lists all enhancements and respective resonance conditions found for each lattice feature geometry. 
Table 4.4 Parameter ranges swept for the equilateral triangle nanopillar geometry.

\begin{tabular}{|c|c|c|}
\hline Parameter & Sweep Range (nm) & Optimal (nm) \\
\hline$a$ & $75-400$ & 265 \\
\hline$l$ & $50-215$ & 50 \\
\hline$h$ & $105-205$ & 165 \\
\hline
\end{tabular}

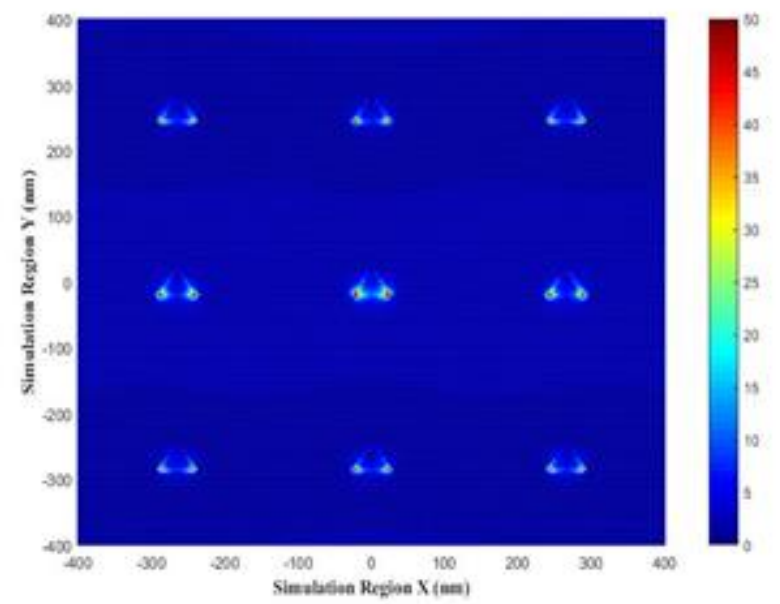

Figure 4.16 Surface-enhanced $E$-field $\left(\left|\mathrm{E} / / \mathrm{E}_{0}\right|\right)$ of the parametrically optimized lattice with a plane wave source emitting at $\lambda=598.202 \mathrm{~nm}$.

Table 4.5 Optimized surface $E$-Field enhancements observed for each pillar geometry.

\begin{tabular}{|c|c|c|}
\hline Pillar Geometry & Surface $\boldsymbol{E}$-Field Enhancement & $\lambda(\mathbf{n m})$ \\
\hline $\begin{array}{c}\text { Non-Optimized } \\
\text { Squares }\end{array}$ & 6 & 565 \\
\hline Optimized Squares & $61 / 30$ & $596.593 / 565$ \\
\hline Cylinders & 63 & 599.7 \\
\hline Triangles & 77 & 598.202 \\
\hline Stars & 24 & 601.205 \\
\hline
\end{tabular}

Presented above was the initial methodology of optimizing various plasmonic lattice feature geometries yielding maximum surface $E$-field enhancement for labeled fluorescence-based biosensing applications. Upon analysis of the surface enhancements of the various plasmonic lattice feature geometries, it was discovered that the equilateral triangular pillar geometry yielded the greatest enhancement observed among all the pillar geometries inspected with an $E$-field enhancement of 77 at $\lambda=598.202 \mathrm{~nm}$. Every optimized plasmonic lattice exhibited a resonant condition within $\pm 5 \mathrm{~nm}$ of $600 \mathrm{~nm}$, irrespective of pillar geometry. 


\subsection{Final Critical Lattice Parameter Optimizations}

Similar to the metal nanostructures catalogued in Table 2.5, the periodic plasmonic lattice features optimized in this section were designed for integration into two biosensor device architectures: 1) free-flow microfluidic systems with analytes suspended in solution over the plasmonic lattice, and 2) systems incorporating antibody affinity sites attached to the nanopillar surfaces where regions of highest $E$-field enhancement exist. Furthermore, the described parametric optimization methodology is extensible to any fixed lattice structure engineered for PEF. Parametric analyses of a periodic plasmonic lattice comprised of cylindrical, equilateral star, square, and equilateral triangular nanopillars using Lumerical's FDTD Solutions were performed with the goal of determining the optimal lattice parameters yielding peak surface $E$-field enhancement. The parameters investigated for the four nanopillars were the following: $a, l$ (or $d$ in the case of the cylinder nanopillars), and $t$ where, as previously mentioned, $a$ is lattice constant, $l$ $(d)$ is feature length (diameter), and $t$ is the Au thickness at the top of the nanopillars (previously referred to as $h$ in the initial parameter optimizations described in Section 4.1). The first parameter a directly impacts the other geometric parameters and fabrication options (i.e., $a$ restricts $l, d$, and $t$ ). Consequently, $l, d$, and $t$ must be based on $a$. The feature basis of the plasmonic lattices constructed and simulated using FDTD were based on the fabrication methodology presented in [187]. In the aforementioned paper, a hydrogen silsesquioxane (HSQ)-based electron beam resist was used due to its chemical composition whereby, after exposure to oxygen plasma, the patterned resist cures into silicon dioxide $\left(\mathrm{SiO}_{2}\right)$. The HSQ-based resist was then spun onto the surface of the silicon ( $\mathrm{Si}$ ) substrate, patterned using EBL with nanometer pattern generation system (NPGS) software, and then cured into $\mathrm{SiO}_{2}$ nanopillars approximately $100 \mathrm{~nm}$ in height. After curing, a 5$\mathrm{nm}$ layer of titanium ( $\mathrm{Ti}$ ) was deposited to ensure the successful bonding of Au with the top of the nanopillars. The material properties and optical constants of the Au and Ti layers used in simulation followed those described in [177].

Following the above fabrication constraints, the FDTD simulation architecture was the following for all four nanopillar geometries: The refractive index $(n)$ of the simulation region was set to 1.497 corresponding to the $n$ of the toluene solution in which the QDs are suspended, a $1 \mu \mathrm{m}$ x $1 \mu \mathrm{m}$ Si base used as the substrate upon which a 3x3 periodic array of $\mathrm{SiO}_{2}$ nanopillars $100 \mathrm{~nm}$ in height each capped with a 5-nm Ti layer were constructed. 
Atop of each 5-nm Ti layer, $t$ was added and swept ranging from $5 \mathrm{~nm}$ up to $105 \mathrm{~nm}$ in $25-\mathrm{nm}$ increments. $l$ and $d$ were swept over a range beginning at $50 \mathrm{~nm}$ up to $200 \mathrm{~nm}$ in $25-\mathrm{nm}$ increments. Finally, $a$ was swept over a range beginning $25 \mathrm{~nm}$ greater than the smallest $l$ and $d$ (i.e., $75 \mathrm{~nm}$ ) up to a maximum value of $400 \mathrm{~nm}\left(\lambda_{\max } / 2\right)$. A TE-polarized plane wave source at normal incidence $(\theta=0 \circ)$ was used to model a uniform ensemble of emitters possessing a 500 -nm bandwidth $(\Delta \lambda)$ ranging from $300 \mathrm{~nm}$ to $800 \mathrm{~nm}$ sampled at a rate of one point per wavelength $\left(S_{\mathrm{R}}\right)$. The parameter sweeps for all four nanopillar geometries began at $t=5 \mathrm{~nm}$ and $a=75 \mathrm{~nm}$. A point monitor was positioned at the top center of $\mathrm{t}\left(t_{z \max }\right)$ of the centermost nanopillar set to record $E$-field data at each wavelength (i.e., 501 frequency points). The simulation environment was discretized in space by establishing a mesh step size $(\mathrm{d} x, \mathrm{~d} y, \mathrm{~d} z)$ of $10 \mathrm{~nm}$ to avoid lengthier run times upwards of 24 hours for each of the 385 simulations per feature geometry. Time was discretized $(\mathrm{d} t)$ in 0.99 fs increments with each simulation set to run for 1,000 fs. The boundary conditions of the simulation region were set to periodic in the positive and negative $x$ - and $y$-directions since both the physical structures and electromagnetic fields were repeated in those directions [192] and PML in the positive and negative $z$-directions. An $X Z$ cross-sectional perspective of the device architecture modeled in FDTD can be seen in Figure 4.17. Table 4.6 details the ranges of all lattice feature parameters swept.

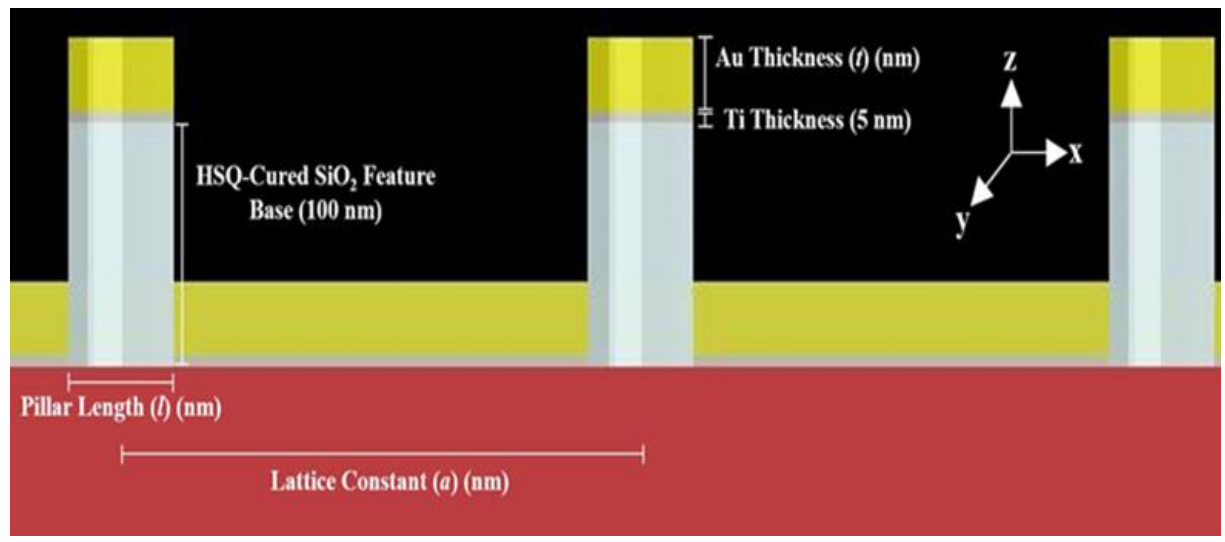

Figure 4.17 XZ cross-sectional perspective of the lattice structure including nanopillar composition and labeled parameters simulated in FDTD.

Table 4.6 Plasmonic lattice parameters swept. Shown in parentheses are the number of data sets recorded from each $a$ and $t$ sweep for each $l$.

\begin{tabular}{|c|c|c|}
\hline Length or Diameter $(\boldsymbol{l}, \boldsymbol{d})(\mathbf{n m})$ & Lattice Constant Sweep $(\boldsymbol{a})(\mathbf{n m})$ & Au Thickness $(\boldsymbol{t})(\mathbf{n m})$ \\
\hline$l_{1}, d_{1}-50$ & $a_{1}: 75-400(14)$ & $t_{1}: 5-105(5)$ \\
\hline$l_{2}, d_{2}-75$ & $a_{2}: 100-400(13)$ & $t_{2}: 5-105(5)$ \\
\hline$l_{3}, d_{3}-100$ & $a_{3}: 125-400(12)$ & $t_{3}: 5-105(5)$ \\
\hline$l_{4}, d_{4}-125$ & $a_{4}: 150-400(11)$ & $t_{4}: 5-105(5)$ \\
\hline$l_{5}, d_{5}-150$ & $a_{5}: 175-400(10)$ & $t_{5}: 5-105(5)$ \\
\hline$l_{6}, d_{6}-175$ & $a_{6}: 200-400(9)$ & $t_{6}: 5-105(5)$ \\
\hline$l_{7}, d_{7}-200$ & $a_{7}: 225-400(8)$ & $t_{7}: 5-105(5)$ \\
\hline
\end{tabular}


Contrary to the initial parametric sweeps described in Section 3.4.1, the following parameter optimization effort did not follow the fixed parameter set outlined in [240]. Instead, each of the investigated parameters were treated independently. This independence resulted in a total of 192,885 total combinations of parameters for the same four nanopillar geometries examined following:

$C_{P}=\left(\sum_{i=1}^{7} a_{l} t_{l}\right) \times S_{R}$

Figures 3.30 through 3.33 show every parameter combination following (188) for all four pillar geometries explored. Shown in Table 3.9 are $a_{l}$ and $t_{l}$ representing the number of datasets recorded for each $l$ shown in parentheses, respectively.

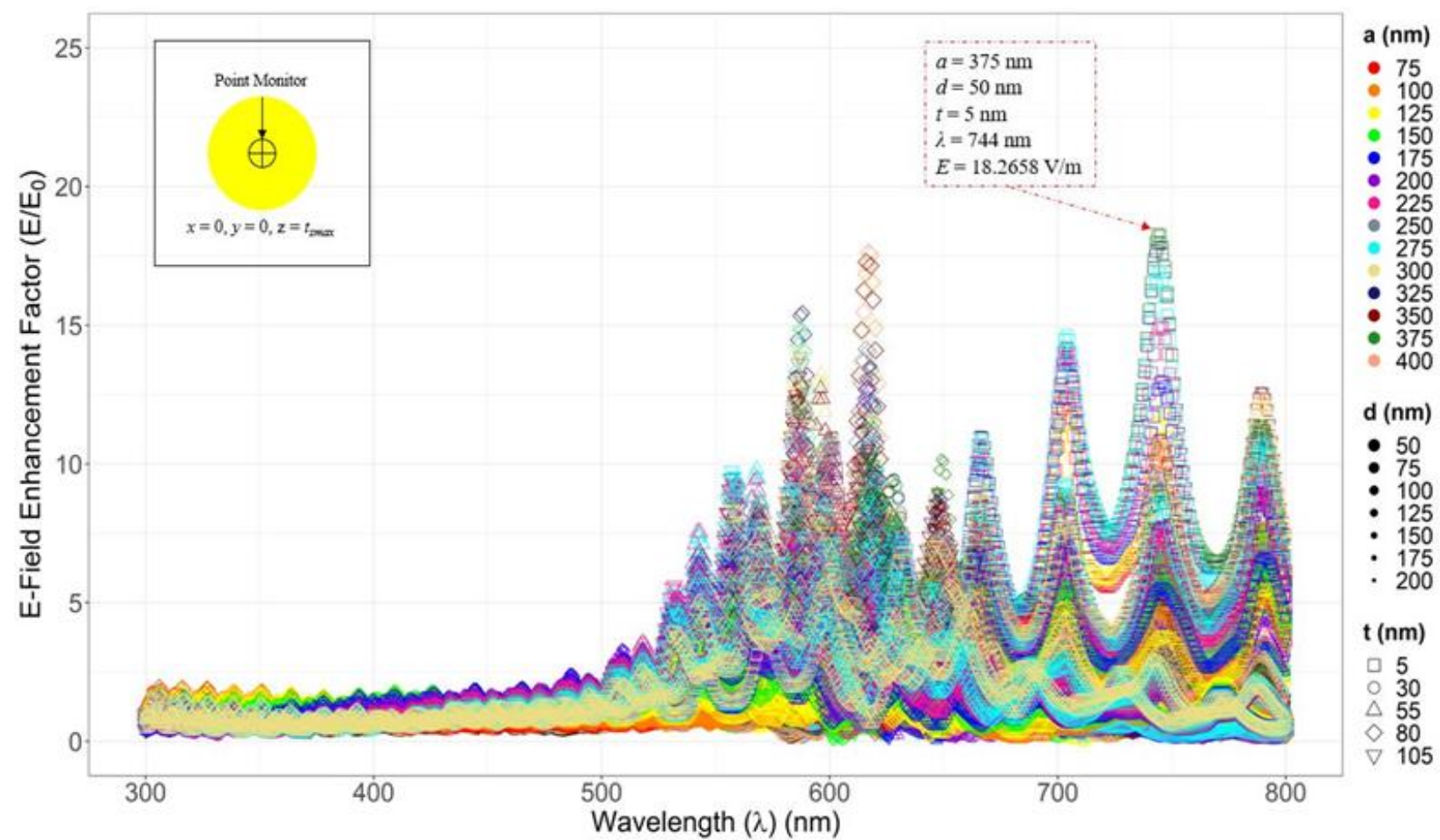

Figure 4.18 Simulated $E$-field enhancement as a function of $\lambda, a, d$, and $t$ from parameter sweeps for the cylinder nanopillar geometry with $\mathrm{d} x$, $\mathrm{d} y$, and $\mathrm{d} z=10 \mathrm{~nm}$. Highlighted in red is the maximum $E$-field enhancement factor of 18.27 found at $\lambda=744 \mathrm{~nm}, a=375 \mathrm{~nm}, d=50 \mathrm{~nm}$, and $t=$ $5 \mathrm{~nm}$. Inlaid is the point monitor located at $t_{\mathrm{zmax}}$ of the center nanopillar in the $3 \times 3$ array where the data was collected. 


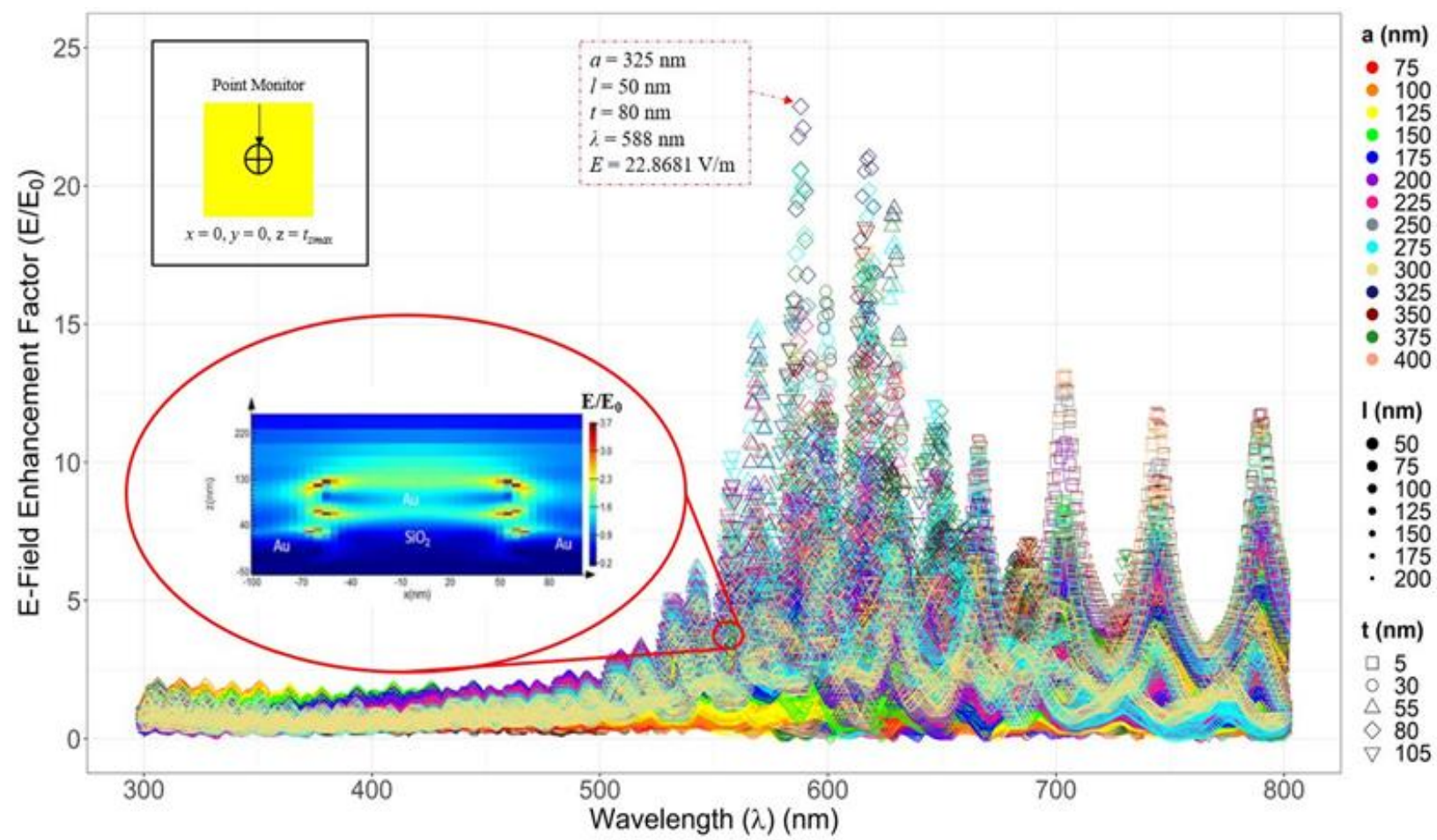

Figure 4.19 Simulated $E$-field enhancement as a function of $\lambda, a, l$, and $t$ from parameter sweeps for the square nanopillar geometry with $\mathrm{d} x$, d $y$, and $\mathrm{d} z=10 \mathrm{~nm}$. Highlighted in red is the maximum $E$-field enhancement factor of 22.87 found at $\lambda=588 \mathrm{~nm}, a=325 \mathrm{~nm}, l=50 \mathrm{~nm}$, and $t=80$ $\mathrm{nm}$. Inlaid is the point monitor located at $t_{\mathrm{zmax}}$ of the center nanopillar in the $3 \times 3$ array where the data was collected. Also included is the result found in [187] verified by these data.

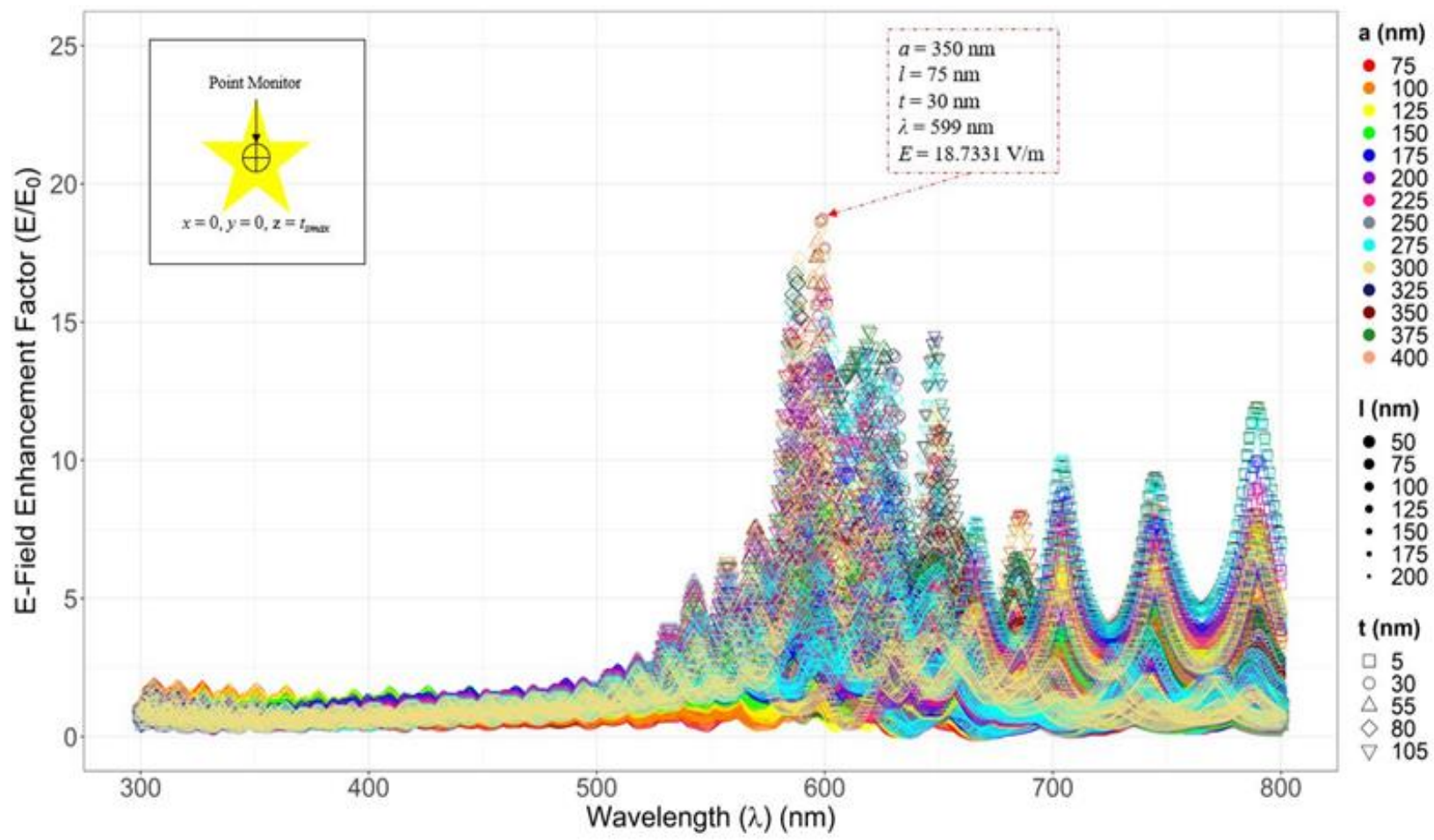

Figure 4.20 Simulated $E$-field enhancement as a function of $\lambda, a, l$, and $t$ from parameter sweeps for the equilateral star nanopillar geometry with $\mathrm{d} x, \mathrm{~d} y$, and $\mathrm{d} z=10 \mathrm{~nm}$. Highlighted in red is the maximum $E$-field enhancement factor of 18.73 found at $\lambda=599 \mathrm{~nm}, a=350 \mathrm{~nm}, l=75 \mathrm{~nm}$, and $t=30 \mathrm{~nm}$. Inlaid is the point monitor located at $t_{\mathrm{zmax}}$ of the center nanopillar in the $3 \times 3$ array where the data was collected. 


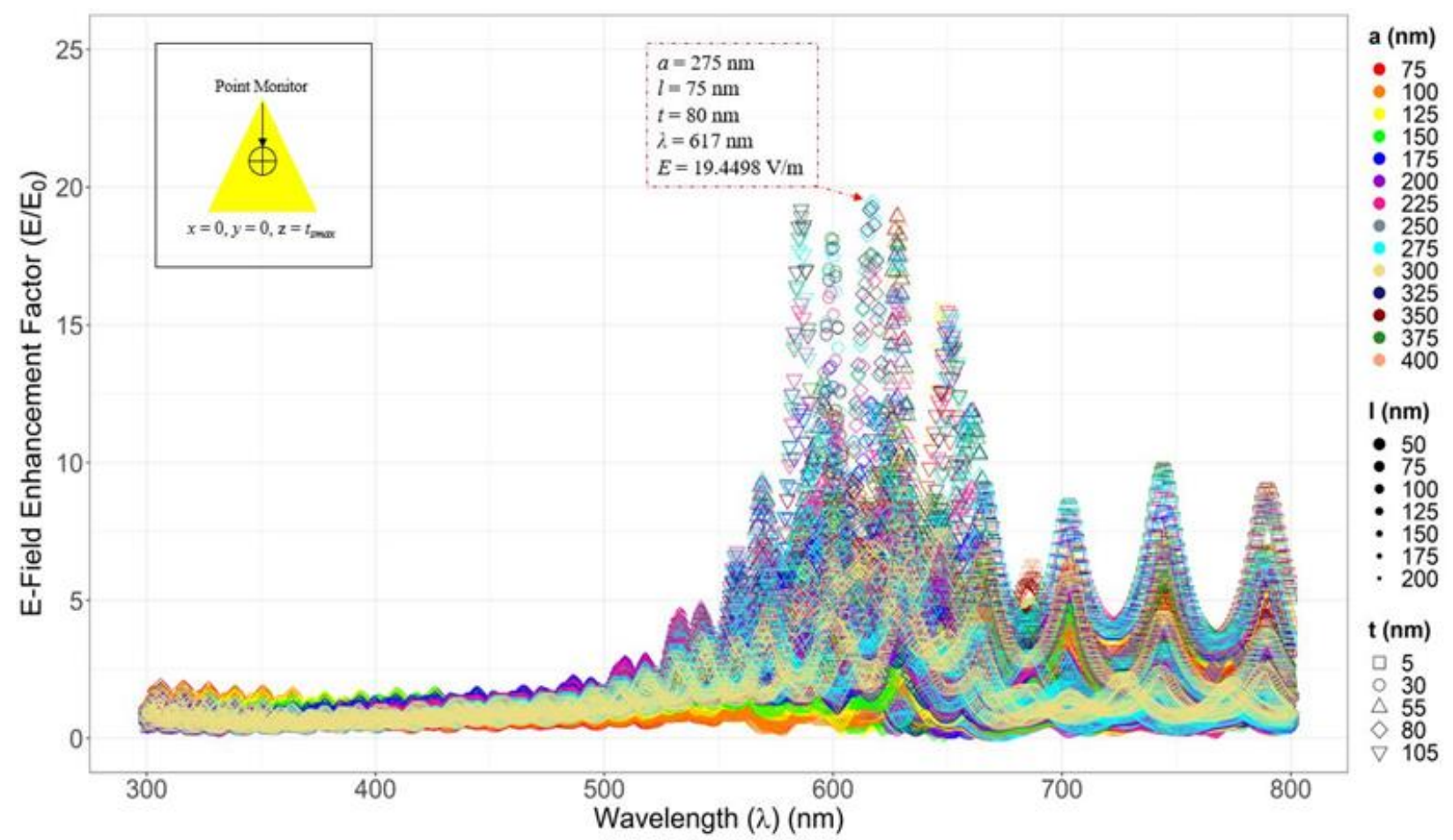

Figure 4.21 Simulated $E$-field enhancement as a function of $\lambda, a, l$, and $t$ from parameter sweeps for the equilateral triangle nanopillar geometry with $\mathrm{d} x, \mathrm{~d} y$, and $\mathrm{d} z=10 \mathrm{~nm}$. Highlighted in red is the maximum $E$-field enhancement factor of 19.45 found at $\lambda=617 \mathrm{~nm}, a=275 \mathrm{~nm}, l=75 \mathrm{~nm}$, and $t=80 \mathrm{~nm}$. Inlaid is the point monitor located at $t_{\mathrm{zmax}}$ of the center nanopillar in the $3 \times 3$ array where the data was collected.

Parametric sweeps of the lattice of cylindrical nanopillars (Figure 4.18) produced an enhancement factor of 18.27 and intensity of 333.79 at $d=50 \mathrm{~nm}, a=375 \mathrm{~nm}, t=5 \mathrm{~nm}$, and $\lambda=$ $744 \mathrm{~nm}$. Parametric sweeps of the lattice of square nanopillars (Figure 4.19) resulted in an enhancement factor of 22.87 and intensity of 523.04 at $l=50 \mathrm{~nm}, a=325 \mathrm{~nm}, t=80 \mathrm{~nm}$, and $\lambda=$ $588 \mathrm{~nm}$. Highlighted within Figure 3.31 is the enhancement factor of approximately 3.7 and intensity of 13.69 at $l=115 \mathrm{~nm}, a=200 \mathrm{~nm}, t=60 \mathrm{~nm}$, and $\lambda=560 \mathrm{~nm}$ previously obtained via FDTD simulation by the Dawson Research Group at West Virginia University [187]. Parametric sweeps of the lattice of star nanopillars (Figure 4.20) saw an enhancement factor of 18.73 and intensity of 350.81 at $l=75 \mathrm{~nm}, a=350 \mathrm{~nm}, t=30 \mathrm{~nm}$, and $\lambda=599 \mathrm{~nm}$. Finally, parametric sweeps of the lattice of triangular nanopillars (Figure 4.21) yielded an enhancement factor of 19.45 and intensity of 378.30 at $l=75 \mathrm{~nm}, a=275 \mathrm{~nm}, t=80 \mathrm{~nm}$, and $\lambda=617 \mathrm{~nm}$. Once more, the plane wave source used for all four parametric optimization sweeps possessed the same $\Delta \lambda$ containing all visible wavelengths. All optimized lattice parameters and resulting enhancements displayed in Figure 4.18 through Figure 4.21 are listed in Table 4.7. 
Table 4.7 Optimal parameters determined from the parametric analyses performed including $E$-field enhancement factors, $E$-field intensities, $\Delta \lambda$, and $\lambda$ where peak enhancement was observed for the four nanopillar feature geometries, respectively.

\begin{tabular}{|c|c|c|c|c|c|c|c|}
\hline $\begin{array}{c}\text { Feature } \\
\text { Geometry }\end{array}$ & $\boldsymbol{l}$ or $\boldsymbol{d}(\mathbf{n m})$ & $\boldsymbol{a}(\mathbf{n m})$ & $\boldsymbol{t}(\mathbf{n m})$ & $\begin{array}{c}\text { Enhancement } \\
\left(|\mathbf{E}| /\left|\mathbf{E}_{\mathbf{0}}\right|\right)\end{array}$ & $\begin{array}{c}\text { Intensity } \\
\left(|\mathbf{E}|^{2} /\left|\mathbf{E}_{\mathbf{0}}\right|^{\mathbf{2}}\right)\end{array}$ & $\boldsymbol{\Delta} \boldsymbol{\lambda}(\mathbf{n m})$ & $\boldsymbol{\lambda}(\mathbf{n m})$ \\
\hline Cylinders & 50 & 375 & 5 & 18.27 & 333.79 & $300-800$ & 744 \\
\hline Squares & 50 & 325 & 80 & 22.87 & 523.04 & $300-800$ & 588 \\
\hline Stars & 75 & 350 & 30 & 18.73 & 350.81 & $300-800$ & 599 \\
\hline Triangles & 75 & 275 & 80 & 19.45 & 378.30 & $300-800$ & 617 \\
\hline
\end{tabular}

Based on these results, single simulations of each plasmonic lattice incorporating the optimal parameters found for each nanopillar geometry were performed, respectively. In each of these four simulations, the mesh accuracy was refined by reducing $\mathrm{d} x, \mathrm{~d} y, \mathrm{~d} z$ to $1.5 \mathrm{~nm}$. In conjunction, $\Delta \lambda$ was reduced from $500 \mathrm{~nm}$ down to $100 \mathrm{~nm}$ sampled at the same $S_{\mathrm{R}}$ (i.e., 101 frequency points) set to include the resonant condition for each nanopillar geometry determined from the parameter sweeps shown in Figure 4.18 through Figure 4.21. A planar monitor was configured to include the entire $3 \times 3$ lattice in all four simulations to determine the updated $\lambda$ (i.e., convergence) where peak surface $E$-field enhancement of the entire lattice was observed. The optimized lattice of cylindrical nanopillars resulted in an enhancement factor of 51.98 and intensity of $2,701.92$ at $\lambda=701 \mathrm{~nm}$.

The optimized lattice of square nanopillars resulted in an enhancement factor of 211.10 and intensity of $44,563.21$ at $\lambda=614 \mathrm{~nm}$. The optimized lattice of star nanopillars resulted in an enhancement factor of 335.39 and intensity of $112,486.45$ at $\lambda=625 \mathrm{~nm}$. Finally, the optimized lattice of triangular nanopillars resulted in an enhancement factor of 251.52 and intensity of $63,262.31$ at $\lambda=648 \mathrm{~nm}$. Figure 4.22 shows each optimized $3 \times 3$ plasmonic lattice at their respective $\lambda$. Table 4.8 summarizes the $E$-field enhancements, $E$-field intensities, $\Delta \lambda$, and $\lambda$ for all four parametrically optimized plasmonic lattice feature geometries.

Table 4.8 Peak $E$-field enhancement factors, $E$-field intensities, $\Delta \lambda$, and $\lambda$ for the four optimized nanopillar feature geometries, respectively.

\begin{tabular}{|c|c|c|c|c|c|c|c|}
\hline $\begin{array}{c}\text { Feature } \\
\text { Geometry }\end{array}$ & $\boldsymbol{l}$ or $\boldsymbol{d}(\mathbf{n m})$ & $\boldsymbol{a}(\mathbf{n m})$ & $\boldsymbol{t}(\mathbf{n m})$ & $\begin{array}{c}\text { Enhancement } \\
\left(\left|\mathbf{E} / / \mathbf{E}_{0}\right|\right)\end{array}$ & $\begin{array}{c}\text { Intensity } \\
\left(|\mathbf{E}|^{2} /\left|\mathbf{E}_{0}\right|^{2}\right)\end{array}$ & $\boldsymbol{\Delta} \boldsymbol{\lambda}(\mathbf{n m})$ & $\boldsymbol{\lambda}(\mathbf{n m})$ \\
\hline Cylinders & 50 & 375 & 5 & 51.98 & $2,701.92$ & $700-800$ & 701 \\
\hline Squares & 50 & 325 & 80 & 211.10 & $44,563.21$ & $550-650$ & 614 \\
\hline Stars & 75 & 350 & 30 & 335.39 & $112,486.45$ & $550-650$ & 625 \\
\hline Triangles & 75 & 275 & 80 & 251.52 & $63,262.31$ & $550-650$ & 648 \\
\hline
\end{tabular}


(a)

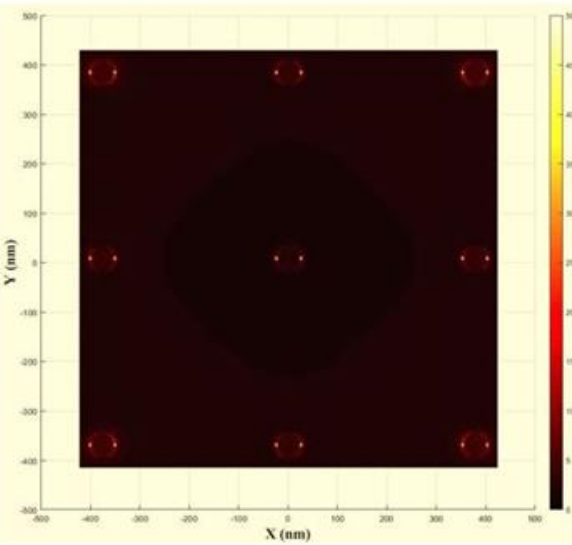

(c)

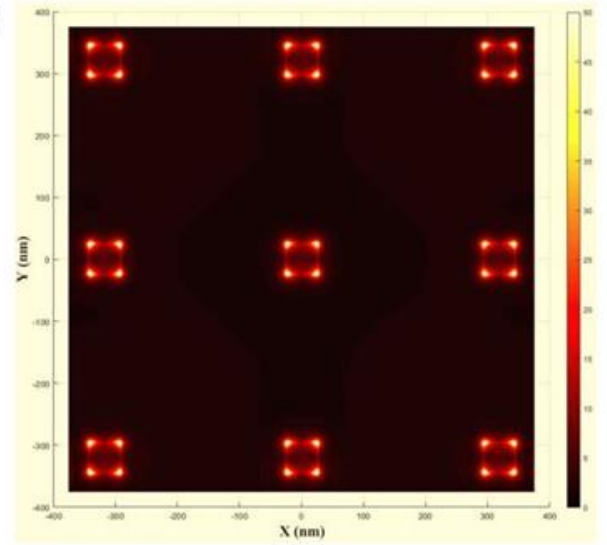

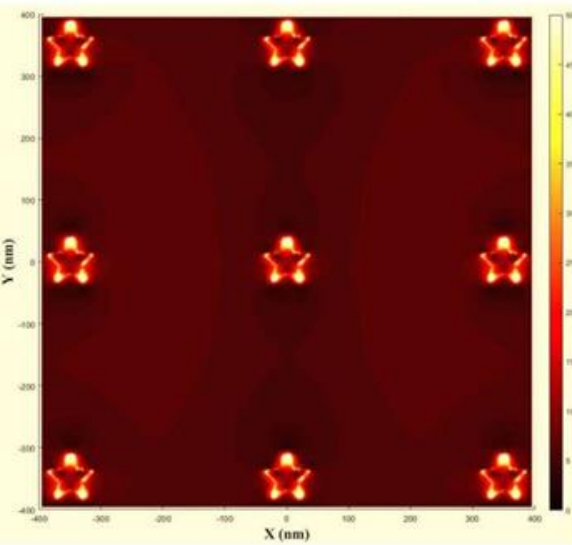

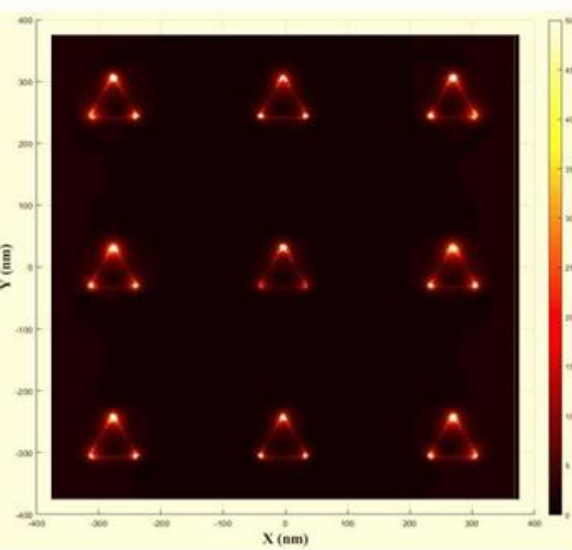

(b)

(d)

Figure 4.22 FDTD planar monitor data $\left(\left|\mathrm{E}_{\mid} / \mathrm{E}_{0}\right|\right)$ for all parametrically optimized lattice structures post-processed using MATLAB: (a) peak surface $E$-field enhancement factor of 51.98 for the lattice of cylindrical nanopillars at $\lambda=701 \mathrm{~nm}$, (b) peak surface $E$-field enhancement factor of 335.39 for the lattice of equilateral star nanopillars at $\lambda=625 \mathrm{~nm}$, (c) peak surface $E$-field enhancement factor of 211.10 for the lattice of square nanopillars at $\lambda=614 \mathrm{~nm}$, and (d) peak surface $E$-field enhancement factor of 251.52 for the lattice of equilateral triangle nanopillars at $\lambda=648$ $\mathrm{nm}$. The same dynamic range of 0 (dark brown) to 50 (white) was used in (a) through (d) for direct comparison. 


\section{Chapter 5}

\section{Conclusion and Future Work}

Chapter 5 outlines a method of fabrication for creating the lattice feature geometries explored in this thesis including the use of a confocal microscope to visualize the fluorescence experimental process shown in Figure 1.6. All findings of this work are summarized, and an applicable fluorophore is selected for use in experimentation involving the optimized plasmonic lattice of star nanopillars since this geometry yielded the highest $E$-field intensity of all lattice geometries investigated. 


\subsection{Conclusion}

The goals of this research were to use FDTD simulation software to extract the optimal lattice parameters of select gold NP lattice geometries yielding peak $E$-field enhancement, compare the resulting $E$-field enhancement factors of each optimized plasmonic lattice geometry at their respective resonance conditions, directly relate these simulated $E$-field enhancement factors to fluorescence enhancement, and select label(s) (if available/where applicable) whose emission spectra overlaps with and/or whose peak lies within the resonance condition of each optimized lattice geometry, respectively.

This thesis presented the parametric optimization of four plasmonic lattice feature geometries using FDTD for integration into visible wavelength fluorescence spectroscopy. It was observed that these $E$-field data resulting from FDTD simulations were localized at the outer edges of the features, consistent with plasmonic literature. Each of the four optimized plasmonic lattice geometries exhibited resonance conditions within the visible regime of the electromagnetic spectrum. The modeled $E$-field intensity of 44,563 at $\lambda=614 \mathrm{~nm}$ for the array of square nanopillars, 63,262 at $\lambda=648 \mathrm{~nm}$ for the array of equilateral triangle nanopillars, and 112,486 at $\lambda=625 \mathrm{~nm}$ for the array of equilateral star nanopillars shown in this paper are greater than all $E$-field intensities outlined in Table 2.5 except for the $E$-field intensity of 133,225 at $\lambda \sim 625 \mathrm{~nm}$ reported in [241] for Au nanostars. Furthermore, the square nanopillar array results previously reported by the Dawson Research Group at West Virginia University in [187] were optimized by over an entire order of magnitude. Although every parameter obtained from this optimization effort adhered to specific dimensional, fabrication, and spectral constraints, the methodology described in this work is extensible to any fixed lattice structure and/or any NP morphology engineered for PEF spectroscopy. The parametric optimization methodology described herein could be furthered improved by incorporating a supercomputer and/or parallel processing to perform all parametric analyses at the highest simulation accuracy currently available in FDTD software (i.e., $\mathrm{d} x, \mathrm{~d} y$, and $\mathrm{d} z=0.25 \mathrm{~nm}$ ) [192]. This higher accuracy feature analysis could then be coupled with machine learning algorithms aimed at discovering other parameter combinations yielding comparable, or greater, $E$-field enhancement factors. The task of obtaining feature geometries for a desired electromagnetic response remains a challenging and time-consuming task [249-251]. In more recent years, nanophotonic devices have been designed via deep-learning and/or neural network algorithms aimed at hyper-parameterization optimization [249-251]. 
Neural networks artificially replicate the way by which the human brain processes different pieces of information and, like the human brain comprised of interconnected neural networks, computers can be programmed to process and learn from a given dataset. Deep-learning algorithms enable the computer to train itself to process and learn from the datasets over which it supervises. The FDTD parametric analyses performed in this work could incorporate such an algorithm to further increase the total number of critical parameter combinations explored. Specifically, a deeplearning and/or neural network algorithm would enable the probing of a much larger parameter space adapting itself as $E$-field simulation data is recorded. Future research efforts will include the fabrication of these optimized plasmonic lattices for physical fluorescence experimentation using commercially available fluorophores possessing emission spectra centered at any of the resulting $\lambda$ depending on the application. Taking the highest intensity of $112,486.45$ at $\lambda=625 \mathrm{~nm}$ yielded by the star nanopillars and referring to Table 2.9, the Rhodamine-B fluorophore would be best suited for use in experimentation since its emission $\lambda$ is closest to the resonance condition of the lattice $(\lambda=627 \mathrm{~nm})$ and possesses a very high $\eta$ of 0.97 .

\subsection{Future Work - Fabrication of the Optimized Plasmonic Lattice}

This section details the equipment and materials needed for fabricating any optimized plasmonic lattice presented in this work.

\subsubsection{Sample Cleaning and Preparation}

Device fabrication begins with the substrate - $100 \mathrm{~mm}$ p-type silicon ( $\mathrm{Si}$ ) wafers from MEMC Electronic Materials Inc. oriented in (100) are cut into $1 \mathrm{~cm} \times 1 \mathrm{~cm}$ squares using a hand diamond scribe. Scribed samples are then degreased in a sonication bath of acetone and methanol separately for five minutes each. Deionized (DI) water is used to rinse the samples of any remaining chemical traces and surface contaminants. Samples are then dried using a nitrogen $(\mathrm{N})$ gun and placed in a $120^{\circ} \mathrm{C}$ oven for 20 minutes to remove any remaining moisture. After baking, samples are removed and allowed to cool to room temperature. 


\subsubsection{Electron Beam Resist}

Hydrogen Silsesquioxane (HSQ), chemical formula $\mathrm{H}_{8} \mathrm{Si}_{8} \mathrm{O}_{12}$, is a spin-on-dielectric material utilized in the electronics industry. Through the recent use of HSQ-based resins, it was discovered that exposure to electron beam energy could cure the material. HSQ-based resins have shown the capability of rendering features below $10 \mathrm{~nm}$. Additionally, subsequent oxygen $\left(\mathrm{O}_{2}\right)$ plasma curing of developed HSQ materials can chemically transform the material to silicon dioxide $\left(\mathrm{SiO}_{2}\right)$. Other electron beam resists require additional steps (e.g., deposition, lift off, etching, etc.) to achieve similar results. As previously described, the chemical properties of HSQ provide an overall reduction in fabrication steps and increase throughput capabilities. Dow Corning's XR-1541-006 HSQ-based electron beam resist is deposited by spin coating with a Laurel Technologies 600 spinner. To obtain the desired 100-nm coating, a spin process of 1200 rpm for 60 seconds at an acceleration of 1740 is required. Immediately after spinning, any remaining solvent is evaporated by placing the coated samples onto a $95^{\circ} \mathrm{C}$ hotplate for four minutes.

\subsubsection{Electron Beam Lithography}

EBL is the process of pattern transfer onto the surface of a substrate by scanning and exposing a layer of electron beam resist via a precisely controlled and focused electron beam. Subsequently, the exposed or non-exposed regions of the resist are removed by a special solvent (i.e., development). EBL grants high-density micro- to nano-scale feature resolution and does not suffer from diffraction limitations characteristic of typical optical lithography methods such as photolithography [199-204, 205-215]. Although EBL does have a wide variety of advantages, it is time consuming for some processes resulting in lower throughput [214-215]. Feature-size variance is one of the highest priorities in using this technique to produce repeatable results; however, critical dimension accuracy is affected by a common issue experienced in the electron beam exposure technique - the proximity effect. During the process of electron beam energy exposure, a number of primary beam electrons contribute to form secondary electrons that are generated by the electron interaction with the materials present [207]. These electrons do not

possess enough energy to completely expose the resist. This leads to the interaction of low-energy secondary electrons with high-energy primary electrons triggering a scattering effect of the primary electrons. 
This scattering phenomenon ultimately results in either the enlargement and/or reduction of the designed feature sizes [208] and affects critical dimension accuracy. The proximity effect is usually observed when writing high-density features or repeating patterns, as reported by several groups [208-213]. The extent of the proximity effect has been shown to relate to the acceleration voltage of the electron beam. At higher acceleration voltages $(\geq 100 \mathrm{kV})$ the effects seem to be worsened. At lower acceleration voltages $(<1 \mathrm{kV})$, the proximity effects are significantly reduced, but with a tradeoff resulting in poor resist sensitivity and longer writing speeds [205-215] (Figure 5.1). The JEOL JSM-7600F SEM system with EBL capabilities was used in conjunction with nanometer pattern generation system (NPGS) software to create the features upon which metal would be deposited.

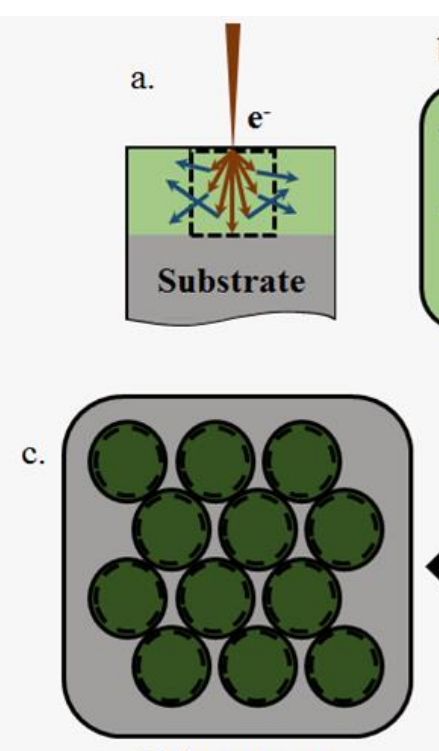

Enlargement

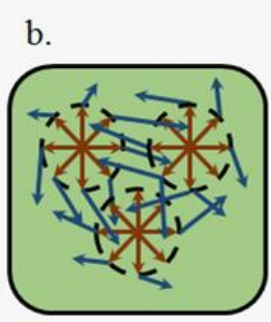

Primary electrons

Second electrons

$\square$ Unexposed resist

I- - $\mathbf{-}$ Designed feature

$\square$ Resolved feature

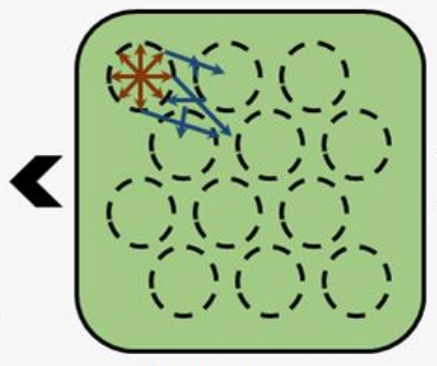

Exposure step

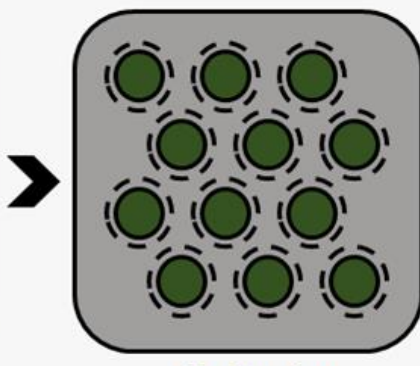

Reduction

Figure 5.1 Illustration of the proximity effect that occurs in EBL a) generation of secondary electrons b) interaction of the secondary electrons with the primary electrons c) graphic of the proximity effect in high-density patterning resulting in either enlargement or reduction of designed features [252].

\subsubsection{Development}

After exposure, resist-coated samples are then placed in a development bath of MF-26A developer heated to $80^{\circ} \mathrm{C}$ for two minutes and 30 seconds. The samples were then lightly rinsed in DI water and dried using an $\mathrm{N}$ gun. Extra caution must be exercised during the moisture removal as features can be blown off by air blasts that are too strong. 


\subsubsection{Oxygen Plasma Ashing}

A March PX-250 plasma asher operating at a power of $300 \mathrm{~W}$ and 300 mTorr for 600 seconds is used to fully expose and cure the patterned features. Developed XR-1541 transforms into $\mathrm{SiO}_{2}$ through $\mathrm{O}_{2}$ plasma exposure. The HSQ-based EBL method performed in this work allows for direct feature curing into $\mathrm{SiO}_{2}$ via $\mathrm{O}_{2}$ plasma which then creates an immediate feature base. Additional processes can be performed on these features for the construction of a wide range of devices with a plethora of applications, namely a plasmonic lattice.

\subsubsection{Metal Deposition}

Titanium (Ti) is first deposited onto the samples using a Kurt J. Leskar electron beam evaporation system to serve as an adhesive between the cured $\mathrm{SiO}_{2}$ patterned features and the gold $\mathrm{Au}$ ) layer. Immediately following $\mathrm{Ti}$ deposition, a layer of Au of a specified thickness is deposited to achieve the plasmonic effect. The full step-by-step metal deposition process is shown below in Figure 5.2.

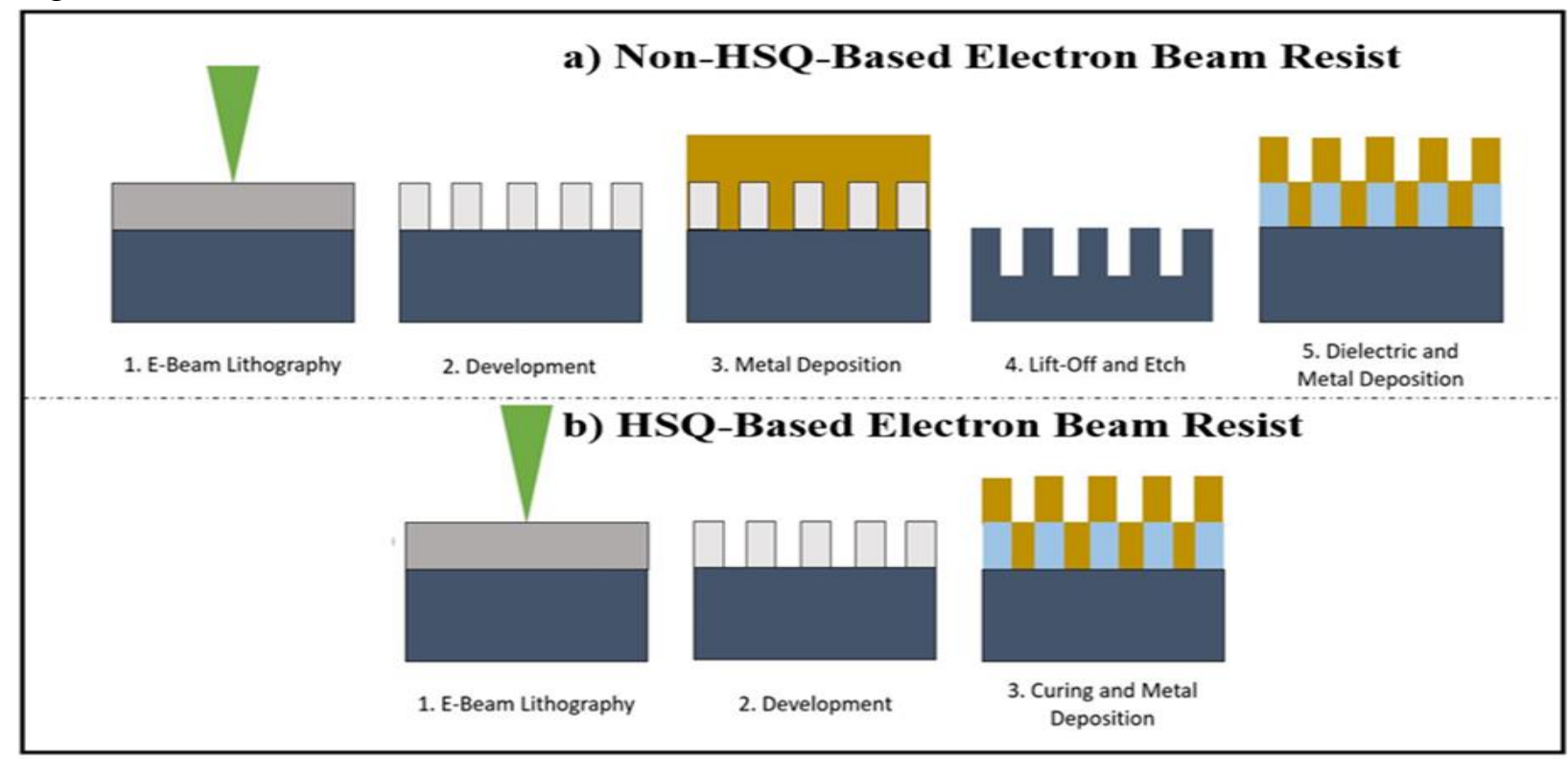

Figure 5.2 Overview of the HSQ- and non-HSQ-based electron beam lithography techniques [252]. 


\subsubsection{Spacer Layer Deposition}

The Temescal BJD 2000 electron beam evaporation system is used for the deposition of a 10 $\mathrm{nm}$ spacer layer of $\mathrm{Si}$ of $\mathrm{SiO}_{2}$ atop the Au layer to reduce quenching. For optimal enhancement, spacer layer thickness should be significantly small (i.e., between $1 \mathrm{~nm}-10 \mathrm{~nm}$ ) [16-17]. Figure 5.3 outlines the entire process flow for both non-HSQ-based and HSQ-based device fabrication.

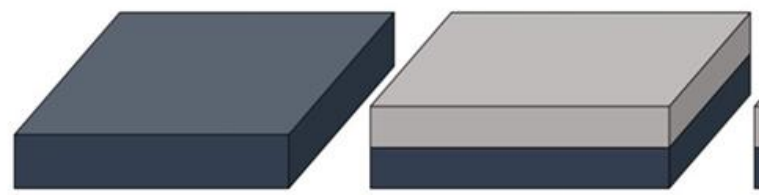

Prep and Clean
Resist Spinning

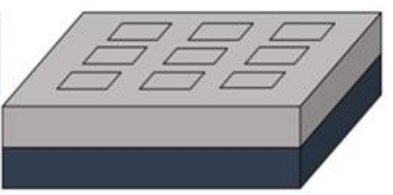

EBL Patterning

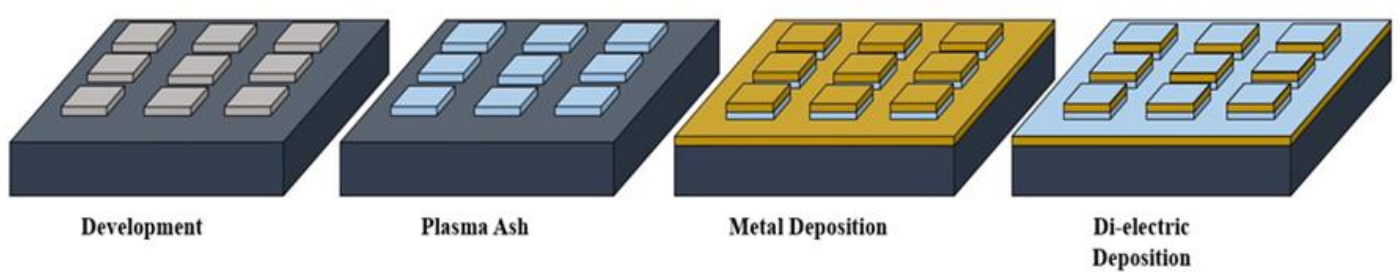

Figure 5.3 Process flow for plasmonic device fabrication using HSQ-based resist including metal and dielectric layer deposition [252].

\subsubsection{Characterization and Experimentation}

Characterization of fluorescence enhancement can be performed using a FITC filtered Ziess optical microscope outfitted with a digital observation camera, lasing source, filter array, and computer imaging software. A diagram of the microscope setup used to visualize a potential biosensor utilizing an optimized plasmonic transducer from this thesis as depicted previously in Figure 1.6 is shown below in Figure 5.4.

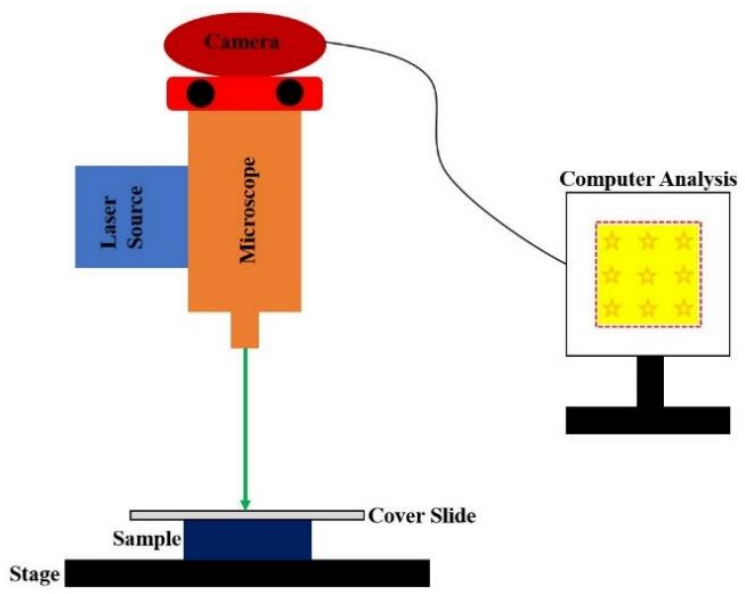

Figure 5.4 Diagram of the characterization set up for testing of the plasmonic lattice (adapted from [252]). 


\section{Chapter 6}

\section{References}

[1] "Biosensor Market is Projected to Touch US\$ 26 Billion by 2022," MarketWatch, 2018. [Online]. Available: https://www.marketwatch.com/press-release/biosensor-market-isprojected-to-touch-us-26-billion-by-2022-2018-11-28. [Accessed 18 July 2019].

[2] "Biosensor Market By Application - Global Forecast by 2018-2024," Market Research Engine, 2018. [Online]. Available: https://www.marketresearchengine.com/biosensormarket-report. [Accessed 18 July 2019].

[3] J. P. Camden, et al., "Controlled Plasmonic Nanostructures for Surface-Enhanced Spectroscopy and Sensing," American Chemical Society, vol. 41, no. 12, pp. 1653-1661, 2008.

[4] A. G. Brolo, et al., "Enhanced Fluorescence from Arrays of Nanoholes in a Gold Film," American Chemical Society, no. 127, pp. 14936-14941, 2005.

[5] S. Das, et al., "Enhancement Mechanism of Fluorescence Intensity in Presence of Plasmonic Nanoparticles," in Proceedings of SPIE, 2015.

[6] E. C. Le Ru, et al., Spectral Profile Modifications In Metal-Enhanced Fluorescence, John Wiley and Sons, Inc, 2010.

[7] J. R. Lakowicz, et al., "Plasmon-controlled fluorescence: A new detection technology," in Proceedings of SPIE, 2006.

[8] J.-F. Li, et al., "Plasmon-enhanced fluorescence spectroscopy," The Royal Society of Chemistry, no. 46, pp. 3962-3979, 2017.

[9] Z. Zhu, et al., "Plasmon-Enhanced Fluorescence in Coupled Nanostructures and Applications in DNA Detection," American Chemical Society Journal of Applied Bio Materials, no. 1, pp. 118-124, 2018.

[10] F. Tam, et al., "Plasmonic Enhancement of Molecular Fluorescence," American Chemical Society Nano Letters, vol. 7, no. 2, pp. 496-501, 2007.

[11] P. Koedrith, et al., "Recent Trends in Rapid Environmental Monitoring of Pathogens and Toxicants: Potential of Nanoparticle-Based Biosensor and Applications," Hindawi Publishing Corporation, vol. 2015, 2014. 
[12] S.-Y. Liu, et al., "Simultaneous Excitation and Emission Enhancement of Fluorescence Assisted by Double Plasmon Modes of Gold Nanorods," Journal of Physical Chemistry, no. 117, pp. 10636-10642, 2013.

[13] P. T. C. So and C. Y. Dong, "Fluorescence Spectrophotometry," in Encyclopedia of Life Sciences, Macmillan Publishers Ltd, Nature Publishing Group, 2002.

[14] S.-H. Chang, et al., "Surface plasmon generation and light transmission by isolated nanoholes and arrays of nanoholes in thin metal films," Optics Express, vol. 13, no. 8, pp. 3150-3165, 2005.

[15] P. Anger, et al., "Enhancement and Quenching of Single-Molecule Fluorescence," Physical Review Letters, vol. 96, no. 11, 2006.

[16] S. Mubeen, et al., "Plasmonic Properties of Gold Nanoparticles Separated from a Gold Mirror by an Ultrathin Oxide," American Chemical Society Nano Letters, no. 12, pp. 20882094, 2012.

[17] N. Ganesh, et al., "Distance dependence of fluorescence enhancement from photonic crystal surfaces," Journal of Applied Physics, vol. 103, no. 8, 2008.

[18] M. Olejnik, et al., "Plasmonic Molecular Nanohybrids-Spectral Dependence of Fluorescence Quenching," International Journal of Molecular Sciences, no. 13, pp. 10181028, 2012.

[19] B. E. A. Saleh and M. C. Teich, Fundamentals of Photonics, John Wiley and Sons Inc., 2007.

[20] M. Born and E. Wolf, Principles of Optics - Electromagnetic Theory of Propagation Interference and Diffraction of Light, Pergamon Press, 1980.

[21] J. Fortin, "Transduction Principles," in Functional Thin Films and Nanostructures for Sensors, Integrated Analytical Systems, Springer Science+Business Media, LLC, 2009, pp. 17-29.

[22] Z. S. Ballard, et al., "Computation Sensing Using Low-Cost and Mobile Plasmonic Readers Designed by Machine Learning," American Chemical Society Nano, no. 11, pp. 2266-2274, 2017.

[23] H. Xu, et al., "Electromagnetic contributions to single-molecule sensitivity in surfaceenhanced Raman scattering," The American Physical Society, vol. 62, no. 3, pp. 4318-4324, 2000 .

[24] K. C. Bantz, et al., "Recent progress in SERS biosensing," Phys. Chem. Chem. Phys, no. 13, pp. 11551-11567, 2011. 
[25] Y. S. Huh, et al., "Surface enhanced Raman spectroscopy and its application to molecular and cellular analysis," Springer Microfluid Nanofluid, no. 6, pp. 285-297, 2009.

[26] D. Graham, "The Next Generation of Advanced Spectroscopy: Surface Enhanced Raman Scattering from Metal Nanoparticles," Angewandte Chemie International Edition, no. 49, pp. 9325-9327, 2010.

[27] S. Pirotta, et al., "Surface-Enhanced Raman Scattering in Purely Dielectric Structures via Bloch Surface Waves," Journal of Physical Chemistry, no. 117, pp. 6821-6825, 2013.

[28] C. L. Haynes, et al., "Surface-Enhanced Raman Spectroscopy," American Chemical Society Journal of Analytical Chemistry, pp. 338-346, 2005.

[29] N. Alfaraj, "A Review of Charge-Coupled Device Image Sensors," ResearchGate, 2017.

[30] M. F. Limonov, et al., "Fano resonances in photonics," Nature Photonics, vol. 11, pp. 543554, 2017.

[31] A. Rosenberg, et al., "Guided resonances in asymmetrical GaN photonic crystal slabs observed in the visible spectrum," Optical Society of America Optics Express, vol. 13, no. 17, pp. 6564-6571, 2005.

[32] K. Li, et al., "High speed e-beam writing for large area photonic nanostructures-a choice of parameters," Nature Scientific Reports, vol. 6, 2016.

[33] Y. Chen and S. Zhang, "Nanofabrication and coloration study of artificial Morpho butterfly wings with aligned lamellae layers," Nature Scientific Reports, no. 5, 2015.

[34] B. M. Hamza, et al., "The Modeling, Fabrication, and Optical Characterization of Silicon and Polymer-based Photonic Crystals for Biosensing Applications," ECS Transactions, vol. 41, no. 38, pp. 1-10, 2012.

[35] S. Dhuey, et al., "Three-dimensional woodpile photonic crystals for visible light applications," Journal of Physics Communications, vol. 1, 2017.

[36] S. G. Johnson, "Photonic Crystals: A Crash Course in Designer Electromagnetism," MIT Applied Mathematics Free Materials Online.

[37] J. Ali, et al., "Biosensors: Their Fundamentals, Designs, Types, and Most Recent Impactful Applications: A Review," Journal of Biosensors and Bioelectronics, vol. 8, no. 1, 2017.

[38] S. GS, et al., "Biosensors: A Modern Day Achievement," Journal of Instrumentation Technology, vol. 2, no. 1, pp. 26-39, 2014.

[39] G. Gauglitz, "Direct optical sensors: principles and selected applications," Journal of Analytical and Bioanalytical Chemistry, vol. 381, pp. 141-155, 2005. 
[40] A. Syahir, et al., "Label and Label-Free Detection Techniques for Protein Microarrays," Microarrays, vol. 4, pp. 228-244, 2015.

[41] B. Spackova, et al., "Optical Biosensors Based on Plasmonic Nanostructures: A Review," in Proceedings of the IEEE, 2016.

[42] M. Bauch, et al., "Plasmon-Enhanced Fluorescence Biosensors: A Review," Springer Plasmonics, vol. 9, no. 4,pp. 781-799, 2013.

[43] A. G. Brolo, "Plasmonics for future biosensors," Nature Photonics, vol. 6, pp. 709-713, 2012.

[44] R. Gordon, et al., "A New Generation of Sensors Based on Extraordinary Optical Transmission," Accounts of Chemical Research, vol. 41, no. 8, pp. 1049-1057, 2008.

[45] S. Kumar, et al., "Nanohole Array-Detected Trapping of Mammalian Mitochondria Enabling Single Organelle Analysis," American Chemical Society Journal of Analytical Chemistry, vol. 87, pp. 11973-11977, 2015.

[46] P. Damborsky, et al., "Optical biosensors," Biochemical Society Essays in Biochemistry, vol. 60, pp. 91-100, 2016.

[47] A. W. Clark, et al., "Plasmonic Split-Ring Resonators as Dichroic Nanophotonic DNA Biosensors," American Chemical Society, vol. 131, pp. 17615-17619, 2009.

[48] H. Im, et al., "Template-Stripped Smooth Ag Nanohole Arrays with Silica Shells for Surface Plasmon Resonance Biosensing," American Chemical Society, vol. 5, no. 8, pp. 6244-6253, 2011.

[49] D. Ramaccia, et al., "Core-Shell Super-Spherical Nanoparticles for LSPR-Based Sensing Platforms," IEEE Journal of Selected Topics in Quantum Electronics, vol. 23, no. 2, 2017.

[50] A. Barik, et al., "Dielectrophoresis-Enhanced Plasmonic Sensing with Gold Nanohole Arrays," American Chemical Society Nano Letters, no. 14, pp. 2006-2012, 2014.

[51] C. T. Ertsgaard, et al., "Dynamic Placement of Plasmonic Hotspots for Super-resolution Surface-Enhanced Raman Scattering," American Chemical Society Nano, vol. 8, no. 10, pp. 10941-10946, 2014.

[52] S. Y. Lee, et al., "High-Fidelity Optofluidic On-Chip Sensors Using Well-Defined Gold Nanowell Crystals," American Chemical Society Journal of Analytical Chemistry, vol. 83, pp. 9174-9180, 2011.

[53] Y.-s. Wu, "Integrated Nanoplasmonic Optical Microfluidics for Label-free Bioassays," California Digital Library UC Berkeley, 2009. 
[54] S. Kumar, et al., "Nanopore-Induced Spontaneous Concentration for Optofluidic Sensing and Particle Assembly," American Chemical Society Journal on Analytical Chemistry, no. 85, pp. 971-977, 2013.

[55] M. E. Stewart, et al., "Nanostructured Plasmonic Sensors," American Chemical Society Chemical Review, vol. 108, no. 2, pp. 494-521, 2008.

[56] Y. Oh, et al., "Recent advances of nanostructure implemented spectroscopic sensors-A brief overview," Applied Spectroscopy Reviews, vol. 51, no. 7-9, pp. 656-668, 2016.

[57] M. Nirshcl, et al., "Review of Transducer Principles for Label-Free Biomolecular Interaction Analysis," Biosensors, vol. 1, pp. 70-92, 2011.

[58] K. De Vos, et al., "Silicon-on-Insulator microring resonator for sensitive and label-free biosensing," Optical Society of America Optics Express, vol. 15, no. 12, pp. 7610-7615, 2007.

[59] A. C. Hillier, et al., "Surface Plasmon Resonance Enhanced Transmission of Light through Gold-Coated Diffraction Gratings," Analytical Chemistry, vol. 80, pp. 3803-3810, 2008.

[60] F. Vollmer and S. Arnold, "Whispering-gallery-mode biosensing: label-free detection down to single molecules," Nature Methods, vol. 5, no. 7, pp. 591-596, 2008.

[61] K. M. Mayer and J. H. Hafner, "Localized Surface Plasmon Resonance Sensors," American Chemcial Society Chemical Reviews, no. 111, pp. 3828-3857, 2011.

[62] K. Q. Le, "Nanoplasmonic Enhancement of Molecular Fluorescence: Theory and Numerical Modeling," Springer Nature, vol. 10, no. 2, pp. 475-482, 2015.

[63] J. Homola, "Present and Future of Surface Plasmon Resonance Biosensors," Analytical and Bioanalytical Chemistry, vol. 377, no. 3, pp. 528-539, 2003.

[64] O. Stranik, et al., "Plasmonic Enhancement of Fluorescence for Sensor Applications," ScienceDirect, vol. 107, pp. 148-153, 2004.

[65] A. J. Tudos, et al., "Handbook of Surface Plasmon Resonance," The Royal Society of Chemistry, 2008, pp. 1-80, pp. 246-394.

[66] M. T. Zin, et al., "Surface-Plasmon-Enhanced Fluorescence from Periodic Quantum Dot Arrays Through Distance Control Using Biomolecular Linkers," Nanotechnology, vol. 20, no. 1, 2008.

[67] W. L. Barnes, et al., "Surface Plasmon Subwavelength Optics," Nature, vol. 424, pp. 824830, 2003. 
[68] S.-H. Yeom, et al., "Highly Sensitive Nano-Porous Lattice Biosensor Based on Localized Surface Plasmon Resonance and Interference," Optics Express, vol. 19, no. 3, pp. 2288222891, 2011.

[69] J. D. Caldwell, et al., "Plasmonic Nanopillar Arrays for Large-Area, High-Enhancement Surface-Enhanced Raman Scattering Sensors," American Chemical Society Nano, vol. 5, no. 5, pp. 4046-4055, 2011.

[70] B. Hecht and L. Novotny, "Surface Plasmons," in Principles of Nano-Optics, Wurzburg, Germany, Cambridge, 2015, pp. 407-450.

[71] E. Ru, et al., "A Quick Overview of Surface-Enhanced Raman Spectroscopy," in Principles of Surface Enhanced Raman Spectroscopy, Wellington, New Zealand, Elsevier, 2008, pp. $1-27$.

[72] S. J. Norton, et al., "Plasmonics Quenching and Enhancement of a Fluorescence Molecule Outside and Inside a Silver Metallic Nanoshell," IEEE Transactions on Nanotechnology, vol. 10, no. 6, pp. 1264-1274, 2011.

[73] L. Yang, et al., "Shell Thickness-Dependent Antibacterial Activity and Biocompatability of Gold@Silver Core-Shell Nanoparticles," The Royal Chemical Society Advances, vol. 7, no. 19, pp. 11355-11361, 2017.

[74] E. M. Purcell, "Spontaneous emission probabilities at radio frequencies," Proceedings of the American Physical Society Physical Review, vol. 69, no. 11 \& 12, p. 681, 1946.

[75] G. Sun, et al., "Plasmonic light-emission enhancement with isolated metal nanoparticles and their coupled arrays," Journal of the Optical Society of America, vol. 25, no. 10, pp. 1748-1755, 2008.

[76] B. Terranova, et al., "Cylindrical channel plasmon resonance for single molecule sensing," Proceedings of SPIE 8994, Photonic and Phononic Properties of Engineered Nanostructures IV, vol. 8994, 2014.

[77] D. A. Boyne, et al., "Plasmonic gold nanostars as optical nano-additives for injection molded polymer composites," IOP Nanotechnology, vol. 28, no. 40, 2017.

[78] D. A. Boyne, et al., "Transverse axis morphological control for tailored gold nanorod (GNR) synthesis," The Royal Chemical Society Advances, vol. 6, no. 63634, 2016.

[79] B. Romeiro and A. Fiore, "Purcell Effect in the Stimulated and Spontaneous Emission Rates of Nanoscale Semiconductor Lasers," IEEE Journal of Quantum Electronics, vol. 54, no. $2,2018$. 
[80] B. Prabowo, et al., "Surface Plasmon Resonance Optical Sensor: A Review on Light Source Technology," Biosensors, vol. 8, no. 80, 2018.

[81] N. A. Cinel, "EBL Fabricated Plasmonic Nanostructures for Sensing Applications," 2013.

[82] T. M. Schmidt, "Engineering of gold and aluminum plasmonic structures: Fabrication and fluorescence enhancement," 2013.

[83] C. Zhang, et al., "Fabricating ordered 2-D nano-structured arrays using nanosphere lithography," Elsevier MethodsX, no. 4, pp. 229-242, 2017.

[84] A. A. Darweesh, et al., "Improved optical enhancement using double-width plasmonic gratings with nanogaps," Chinese Laser Press Photon. Res., vol. 4, no. 5, pp. 173-179, 2016.

[85] M. A. El-Sayed, "Some Interesting Properties of Metals Confined in Time and Nanometer Space of Different Shapes," Accounts of Chemical Research, vol. 34, no. 4, pp. 257-264, 2001.

[86] Y. Xu, et al., "Large scalable and compact hybrid plasmonic nanoantenna array," SPIE Optical Engineering, vol. 57, no. 8, 2018.

[87] J. Cao, et al., "Comparison of Surface Plasmon Resonance and Localized Surface Plasmon Resonance-based optical fibre sensors," Journal of Physics: Conference Series Sensors \& their Applications XVI, no. 307, 2011.

[88] S. T. Kochuveedu, et al., "A study on the mechanism for the interaction of light with noble metal-metal oxide semiconductor nanostructures for various photophysical applications," The Royal Chemical Society Reviews, no. 42, pp. 8467-8493, 2013.

[89] S. Lal, et al., "Nano-optics from sensing to waveguiding," Nature Photonics, vol. 1, pp. 641-648, 2007.

[90] N. Berkovitch, et al., "Nano-plasmonic antennas in the near infrared regime," Journal of Physics: Condensed Matter, no. 24, 2012.

[91] M. Li, et al., "Plasmon-enhanced optical sensors: a review," The Royal Society of Chemistry Analyst, vol. 140, pp. 386-406, 2015.

[92] S. A. O. Olson, et al., "Plasmonic Cup Resonators for Single-Nanohole-Based Sensing and Spectroscopy," The American Chemical Society Photonics, no. 3, pp. 1202-1207, 2016.

[93] S. A. Maier, Plasmonics: Fundamentals and Applications, New York, New York: Springer Science+Business Media LLC, 2007. 
[94] M. Dowran, et al., "Quantum-enhanced plasmonic sensing," Journal of the Optical Society of America Optica, vol. 5, no. 5, pp. 628-633, 2018.

[95] M. I. Stockman, et al., "Roadmap on Plasmonics," IOP Journal of Optics, no. 20, 2018.

[96] I-C. Chen and H.-H. Lin, "Study of the interaction between Gold Nanoparticles and Rose Bengal Fluorophores with Silia Spacers by Time-Resolved Fluorescence Spectroscopy," The Journal of Physical Chemistry, no. 119, pp. 26663-26671, 2015.

[97] P. Zheng, et al., "Tailoring plasmonic properties of gold nanohole arrays for surfaceenhanced Raman scattering," The Royal Society of Chemistry Chem. Phys., no. 17, pp. 21211-21219, 2015.

[98] D. Yoo, et al., "Template-Stripped Tunable Plasmonic Devices on Stretchable and Rollable Substrates," The American Chemical Society Nano, vol. 9, no. 11, pp. 10647-10654, 2015.

[99] M. Tabatabaei, et al., "Tunable 3D Plasmonic Cavity Nanosensors for Surface-Enhanced Raman Spectroscopy with Sub-femtomolar Limit of Detection," American Chemical Society Photonics, no. 2, pp. 752-759, 2015.

[100] D. A. Boyne and M. H. Griep, "Decorated Core-Shell Architectures: Influence of the Dimensional Properties on Hybrid Resonances," Springer Science+Business Media Plasmonics, 2017.

[101] J. Feis, et al., "Surface plasmon polaritons sustained at the interface of a nonlocal metamaterial," American Physical Society Physical Review, vol. B, no. 98, 2018.

[102] H. Raether, Surface Plasmons on Smooth and Rough Surfaces and on Gratings Springer Tracts in Modern Physics, Springer Berlin, 1988.

[103] A. V. Zayats, et al., "Nano-optics of surface plasmon polaritons," in Physics Reports, Elsevier, 2005, pp. 131-314.

[104] A. V. Zayats and I. Smolyaninov, "Pure and Applied Optics," Journal of Optics A, vol. 5, no. S16, 2003.

[105] H. A. Atwater and A. Polman, "Plasmonics for improved photovoltaic devices," Nature Materials, vol. 9, pp. 205-213, 2010.

[106] H. Horvath, "Gustav Mie and the scattering and absorption of light by particles: Historic developments and basics," Elsevier Journal of Quantitative Spectroscopy \& Radiative Transfer, no. 110, pp. 787-799, 2009.

[107] B. Braun, et al., "Cellulosic Nanowhiskers: Theory and Application of Light Scattering from Polydisperse Spheroids in the Rayleigh-Gans-Debeye Reigme," American Chemical Society Biomacromolecules, no. 9, pp. 1255-1263, 2008. 
[108] H. M. G. Wassel, et al., "Opportunities and Challenges of using Plasmonic Components in Nanophotonic Architectures," IEEE, 2012.

[109] E. J. R. Vesseur, et al., "Broadband Purcell enhancement in plasmonic ring cavities," American Physical Society Physical Review B, no. 82, 2010.

[110] J. R. Lakowicz, "Radiative decay engineering 5: metal-enhanced fluorescence and plasmon emission," NIH Analytical Biochemistry, vol. 337, no. 2, pp. 171-194, 2005.

[111] P. Bharadwaj and L. Novotny, "Spectral dependence of single molecule fluorescence enhancement," Optical Society of America Optics Express, vol. 15, no. 21, pp. 1426614274, 2007.

[112] M. G. Williams, et al., "Rapid synthesis of high purity gold nanorods via microwave irradiation," IOP Materials Research Express, no. 4, 2017.

[113] P. Hanarp, et al., "Optical Properties of Short Range Ordered Arrays of Nanometer Gold Disks Prepared by Colloidal Lithography," American Chemical Society Journal of Physical Chemistry, no. 107, pp. 5768-5772, 2003.

[114] K. Hanson, "Emission Quantum Yield".

[115] S. Hughes, et al., "Quantum dynamics of two quantum dots coupled through localized plasmons: An intuitive and accurate quantum optics approach using quasinormal modes," American Physical Society Physical Review, vol. B, no. 92, 2015.

[116] S. Stobbe, et al., "Frequency dependence of the radiative decay rate of excitons in selfassembled quantum dots: Experiment and theory," American Physical Society Physical Review B, no. 80, 2009.

[117] M. K. Dezfouli and S. Hughes, "Regularized quasinormal modes for plasmonic resonators and open cavities," American Physical Society Physical Review B, no. 97, 2018.

[118] A. F. van Driel, et al., "Statistical analysis of time-resolved emission from ensembles of semiconductor quantum dots: interpretation of exponential decay models," 2007.

[119] R. Fitzpatrick, "Plasma Frequency," $31 \quad 3$ 2011. [Online]. Available: http://farside.ph.utexas.edu/teaching/plasma/Plasmahtml/node6.html. [Accessed 26 July 2019].

[120] "Detectors: CCDs for Light-Science Applications," The Marketplace of the Industry Photonics buyers' guide - Photonics Media, 2018. [Online]. Available: https://www.photonics.com/Article.aspx?AID=25141. [Accessed 26 July 2019].

[121] "Advantages of CCD Cameras," Universe Kogaku America Inc., 2019. [Online]. Available: https://www.universeoptics.com/advantages-of-ccd-cameras/. [Accessed 26 July 2019]. 
[122] M. Kuttge, et al., "Ultrasmall Mode Volume Plasmonic Nanodisk Resonators," American Chemical Society Nano Letters, vol. 10, no. 5, pp. 1537-1541, 2010.

[123] P. T. Kristensen, "Modes and Mode Volumes of Leaky Optical Cavities and Plasmonic Nanoresonators," American Chemical Society Photonics, vol. 1, pp. 2-10, 2012.

[124] A. Faraon, et al., "Resonant enhancement of the zero-phonon emission from a colour centre in a diamond cavity," Nature Photonics, vol. 5, pp. 301-305, 2011.

[125] E. Dulkeith, et al., "Gold Nanoparticles Quench Fluorescence by Phase Induced Radiative Rate Suppression," American Chemical Society Nano Letters, vol. 5, no. 4, pp. 585-589, 2005.

[126] A. L. Demming, et al., "Plasmon resonances on metal tips: Understanding tip-enhanced Raman scattering," American Institute of Physics Journal of Chemical Physics, no. 122, 2005.

[127] "Fluorescence Excitation and Emission Fundamentals," UCI Department of Chemistry.

[128] C. A. Balanis, Antenna Theory, Hoboken, NJ: Wiley, 2005.

[129] T. H. Taminiau, et al., "Enhanced directional excitation and emission of single emitter by nano-optical Yagi-Uda antenna," Optics Express, vol. 16, no. 14, pp. 10858-10866, 2008.

[130] D. Dregely, et al., "3D optical Yagi-Uda nanoantenna array," Nature Communications, vol. 2, pp. 1-7, 2011.

[131] M. Bauch and J. Dostalek, "Collective localized surface plasmons for high performance fluorescence biosensing," Optics Express, vol. 21, no. 17, pp. 20470-20483, 2013.

[132] K. Toma, et al., "Long range surface plasmon-coupled fluorescence emission for biosensor applications," Optics Express, vol. 19, no. 12, pp. 11090-11099, 2011.

[133] D. J. Wu, et al., ""Hot spots" induced near-field enhancements in Au nanoshell and Au nanoshell dimer," Applied Physics B-Lasers Opt., vol. 97, no. 2, pp. 497-503, 2009.

[134] A. Kinkhabwala, et al., "Large single-molecule fluorescence enhancements produced by a bowtie nanoantenna," Nature Photonics, vol. 3, no. 11, pp. 654-657, 2009.

[135] A. M. Kern and O. J. F. Martin, "Excitation and reemission of molecules near realistic plasmonic nanostructures," Nano Letters, vol. 11, no. 2, pp. 482-487, 2011.

[136] D. Punj, et al., "A plasmonic 'antenna-in-a-box' platform for enhanced single-molecule analysis at micromolar concentrations," Nature Nanotechnology, vol. 8, no. 7, pp. 512-516, 2013. 
[137] J. Parsons, et al., "Localized surface-plasmon resonances in periodic nondiffracting metallic nanoparticle and nanohole arrays," Physical Reviews B, vol. 79, no. 7, 2009.

[138] F. Xie, et al., "Nanoscale control of Ag nanostructures for plasmonic fluorescence enhancement of near-infrared dyes," Nano Res., vol. 6, no. 7, pp. 496-510, 2013.

[139] H. Aouani, et al., "Bright unidirectional fluorescence emission of molecules in a nanoaperture with plasmonic corrugations," Nano Letters, vol. 11, no. 2, pp. 637-644, 2011.

[140] H. Aouani, et al., "Plasmonic antennas for directional sorting of fluorescence emission," Nano Letters, vol. 11, no. 6, pp. 2400-2406, 2011.

[141] P. G. Etchegoin, et al., "An analytic model for the optical properties of gold," American Institute of Physics Journal of Chemical Physics, vol. 125, no. 189901, pp. 125-127, 2006.

[142] J. A. Fan, et al., "Self-assembled plasmonic nanoparticle clusters," Science, vol. 328, no. 5982, pp. 1135-1138, 2010.

[143] J. W. Attridge, et al., "Sensitivity enhancement of optical immunosensors by the use of surface plasmon-enhanced resonance fluoroimmunoassay," Biosensors Bioelectronics, vol. 6, no. 3, pp. 201-214, 1991.

[144] A. E. Vasdekis and G. P. J. Laporte, "Enhancing single molecule imaging in optofluidics and microfluidics," International Journal of Molecular Science, no. 12, pp. 5135-5156, 2011.

[145] K. Tawa, et al., "Sensitive bioimaging in microfluidic channels on the plasmonic substrate: simple biosensor with extended dynamic range," Sensors Actuators B-Chem., vol. 157, no. 2, pp. 703-709, 2011.

[146] E. Hwang, et al., "Surface plasmon polariton enhanced fluorescence from quantum dots on nanostructured metal surfaces," Nano Letters, vol. 10, no. 3, pp. 813-820, 2010.

[147] K. Tawa, et al., "Optical microscopic observation of fluorescence enhanced by gratingcoupled surface plasmon resonance," Optics Express, vol. 16, no. 13, pp. 9781-9790, 2008.

[148] Y. Jiang, et al., "Surface plasmon enhanced fluorescence of dye molecules on metal grating films," Journal of Physical Chemistry C, vol. 115, no. 25, pp. 12636-12642, 2011.

[149] P. Bharadwaj a. L. Novotny, "Plasmon-enhanced photoemission from a single Y(3)N@C(80) fullerene," Journal of Physical Chemistry C, vol. 114, no. 6, pp. 7444-7447, 2010 .

[150] R. Gill, et al., "Silver nanoparticle aggregates as highly efficient plasmonic antennas for fluorescence enhancement," Journal of Physical Chemistry C, vol. 116, no. 31, pp. 1668716693, 2012. 
[151] M. Schmelzeisen, et al., "Fluorescence enhancement from individual plasmonic gap resonances," American Chemical Society Nano, vol. 4, no. 6, pp. 3309-3317, 2010.

[152] Y. Fu, et al., "Large enhancement of single molecule fluorescence by coupling to hollow silver nanoshells," Chemical Communications, vol. 48, no. 78, pp. 9726-9728, 2012.

[153] A. R. Guerrero, et al., "Experimental confirmation of local field enhancement determining far-field measurements with shell-isolated silver nanoparticles," Small, vol. 8, no. 19, pp. 2964-2967, 2012.

[154] H. W. Yoo, et al., "The fabrication of highly ordered silver nanodot patterns by platinum assisted nanoimprint lithography," Nanotechnology, vol. 22, no. 9, 2011.

[155] W. H. Zhang, et al., "Giant and uniform fluorescence enhancement over large areas using plasmonic nanodots in 3D resonant cavity nanoantenna by nanoimprinting," Nanotechnology, vol. 23, no. 22, 2012.

[156] Y. M. Yu, et al., "The detection of DNA hybridization on phosphorus dendrimer multilayer films by surface plasmon field enhanced-fluorescence spectroscopy," Langmuir, vol. 25, no. 23, pp. 13680-13684, 2009.

[157] C. Huber, et al., "Heterotetramers formed by an S-layer streptavidin fusion protein and corestreptavidin as a nanoarrayed template for biochip development," Small, vol. 2, no. 1, pp. 142-150, 2006.

[158] K. Aslan, et al., "Metal-enhanced fluorescence-based RNA sensing," American Chemical Society, vol. 128, no. 13, pp. 4206-4207, 2006.

[159] J. R. Lakowicz, et al., "Directional surface plasmon-coupled emission: a new method for high sensitivity detection," Biochemical Biophys. Res. Comm., vol. 307, no. 3, pp. 435439, 2003.

[160] K. Tawa, et al., "An application of a plasmonic chip with enhanced fluorescence to a simple biosensor with extended dynamic range," Sensors Actuators B-Chem., vol. 157, no. 2, pp. 703-709, 2011.

[161] J. S. Yuk, et al., "Signal enhancement of surface plasmon-coupled emission (SPCE) with the evanescent field of surface plasmonc on a bimetallic parabaloid biochip," Biosensors Bioelectronics, vol. 26, no. 7, pp. 3213-3218, 2011.

[162] R. Nooney, et al., "Enhancing the analytical performance of immunoassays that employ metal-enhanced fluorescence," Analytical Bioanalytical Chemistry, vol. 396, no. 3, pp. 1127-1134, 2010. 
[163] L. Zhou, et al., "Enhancement of immunoassay's fluorescence and detection sensitivity using three-dimensional plasmonic nano-antenna-dots array," Analytical Chemistry, vol. 84, pp. 4489-4495, 2012.

[164] Y. Wang, et al., "Prostate specific antigen biosensor based on long range surface plasmonenhanced fluorescence spectroscopy and dextran hydrogel binding matrix," Analytical Chemistry, vol. 81, no. 23, pp. 9625-9632, 2009.

[165] Y.-F. Chang, et al., "Discrimination of breast cancer by measuring prostate-specific antigen levels in women's serum," Analytical Chemistry, vol. 83, no. 13, pp. 5324-5328, 2011.

[166] H. Szmacinski, et al., "Time-resolved fluorometric method for one-step immunoassays using plasmonic nanostructures," Journal of Physical Chemistry C Nanomatter Interfaces, vol. 114, no. 16, pp. 7236-7241, 2010.

[167] K. Aslan and T. A. J. Grell, "Rapid and sensitive detection of troponin I in human whole blood samples by using silver nanoparticle films and microwave heating," Clinical Chemistry, vol. 57, no. 5, pp. 746-752, 2011.

[168] A. Scholten, et al., "A mixed alkanethiol based immunosensor for surface plasmon fieldenhanced fluorescence spectroscopy in serum," Analyst, vol. 138, 2013.

[169] Y.-F. Chang, et al., "The utility of a high-throughput scanning biosensor in the detection of the pancreatic cancer marker ULBP2," Biosensors Bioelectronics, vol. 43, pp. 232-237, 2013.

[170] Y. Wang, et al., "Biosensor for the detection of aflatoxin M1 in milk based on long range surface plasmon enhanced fluorescence spectroscopy," Biosensors Bioelectronics, vol. 24, no. 7, pp. 2264-2267, 2009.

[171] C. J. Huang, et al., "Long range surface plasmon-enhanced fluorescence spectroscopy biosensor for ultrasensitive detection of E. coli O157:H7," Analytical Chemistry, vol. 83, no. 3, pp. 674-677, 2011.

[172] J. C. Huang, et al., "Detection of severe acute respiratory syndrome (SARS) coronavirus nucleocapsid protein in human serum using localized surface plasmon coupled fluorescence fiber-optic biosensor," Biosensors Bioelectronics, vol. 25, no. 2, pp. 320-325, 2009.

[173] Y.-F. Chang, et al., "Detection of swine-origin influenza A (H1N1) viruses using localized surface plasmon coupled fluorescence fiber-optic biosensor," Biosensors Bioelectronics, vol. 26, no. 3, pp. 1068-1073, 2010.

[174] "Overview of Fluorescence Excitation and Emission Fundamentals," Olympus, M. Abramowitz and M. W. Davidson, [Online]. Available: https://www.olympuslifescience.com/en/microscope-resource/primer/lightandcolor/fluoroexcitation/. [Accessed 26 July 2019]. 
[175] D. Darvill, et al., "Plasmonic fluorescence enhancement by metal nanostructures: shaping the future of bionanotechnology," Phys. Chem. Chem. Phys, vol. 15, pp. 15709-15726, 2013.

[176] P. B. Johnson and R. W. Christy, "Optical Constants of Noble Metals," Physical Review B, no. 6, pp. 4370-4379, 1972.

[177] W. M. Hanyes, et al., CRC Handbook of Chemistry and Physics, Boca Raton, FL: CRC Press, 2016.

[178] A. I. Dragan, et al., "Ultra-fast pg/ml anthrax toxin (protective antigen) detection assay based on microwave-accelerated metal-enhanced fluorescence," Analytical Biochemistry, no. 425 , pp. 54-61, 2012.

[179] D. Samanta and A. Sarkar, "Immobilization of bio-macromolecules on self-assembled monolayers: methods and sensor applications," The Royal Society of Chemistry Review, no. 40, pp. 2567-2590, 2011.

[180] A. Mateescu, et al., "Thin Hydrogel Films for Optical Biosensor Applications," Membranes, no. 2, pp. 40-69, 2012.

[181] "What are quantum dots?," nanowerk, 2019. [Online]. Available: https://www.nanowerk.com/what_are_quantum_dots.php. [Accessed 29 July 2019].

[182] "Qdot Probes Technology Overview," ThermoFisher Scientific, [Online]. Available: https://www.thermofisher.com/us/en/home/brands/molecular-probes/key-molecularprobes-products/qdot/technology-overview.html. [Accessed 29 July 2019].

[183] Protein Biology Resource Library - Pierce Protein Methods, "Fluorescent Probes," ThermoFisher Scientific, [Online]. Available: https://www.thermofisher.com/us/en/home/life-science/protein-biology/protein-biologylearning-center/protein-biology-resource-library/pierce-protein-methods/fluorescentprobes.html\#4. [Accessed 29 July 2019].

[184] NN-LABS, LLC., NNCrystal, US Corporation, 2014. [Online]. Available: https://nnlabs.com/. [Accessed 30 July 2019].

[185] K. Thorn, "Fluorescence Microscopy: Small Molecule Probes".

[186] A. Kadiyala and J. M. Dawson, "Modeling of Novel Hybrid Photonic Crystal Structures Involving Cured Hydrogen Silsesquioxane Pillars for Improving the Light Extraction in Light-Emitting Diodes," in Proceedings of SPIE, 2016.

[187] A. Gadde, et al., "Fabrication of Two-Dimensional Visible Wavelength Nanoscale Plasmonic Structures Using Hydrogen Silsesquioxane Based Resist," in Proceedings of SPIE, 2015. 
[188] M. Abramowitz and I. A. Stegun, "Riccati-Bessel Functions," in Handbook of Mathematical Functions with Formulas, Graphs, and Mathematical Tables, New York, NY, Dover Books on Mathematics, 1972, p. 445.

[189] A. Taflove, "A Perspective on the 40-Year History of FDTD Computational Electrodynamics," Applied Computational Electromagnetics Society Journal, vol. 22, no. 1, pp. 1-21, 2006.

[190] S. D. Gedney, Introduction to the Finite-Difference Time-Domain (FDTD) Method for Electromagnetics, Morgan \& Claypool Publishers, 2011.

[191] V. Demir, "Simulation of Electromagnetic Fields: The Finite-Difference Time-Domain (FDTD) Method and Its Applications," DeKalb, IL, 2007.

[192] Lumerical FDTD, "Knowledge Base," Lumerical, Inc., 2019. [Online]. Available: https://kb.lumerical.com/solvers_finite_difference_time_domain.html. [Accessed 25 August 2019].

[193] K. S. Yee, "Numerical Solution of Initial Boundary Value Problems Involving Maxwell's Equations in Isotropic Media," IEEE Transactions on Antennas and Propagation, pp. 302307, 1966.

[194] C. R. Nave, "Maxwell's Equations," HyperPhysics, 2017. [Online]. Available: http://hyperphysics.phy-astr.gsu.edu/hbase/electric/maxeq.html. [Accessed 25 August 2019].

[195] Y. ZhiLin, et al., "FDTD for plasmonics: Applications in enhanced Raman spectroscopy," Chinese Science Bulletin Special Topic on Plasmonics, vol. 55, no. 24, pp. 2635-2642, 2010.

[196] A. C. Lesina, et al., "On the convergence and accuracy of the FDTD method for nanoplasmonics," Optical Society of America Optics Express, vol. 23, no. 8, pp. 1048110497, 2015.

[197] R. Courant, et al., "On the partial difference equations of mathematical physics," IBM Journal of Research and Development, vol. 11, no. 2, pp. 215-234, 1967.

[198] R. Merlin, "Pinholes Meet Fabry-Perot: Perfect and Imperfect Transmission of Waves through Small Aperatures," American Physical Society Physical Review X, vol. 2, no. 3, 2012 .

[199] H. Levinson, Principles of Lithography, 3rd Edition, SPIE Press Monograph, Vol. PM198, 2011. 
[200] D. Qin, et al., "Microfabrication, microstructures, and microsystems," Topics in Current Chemistry, no. 194, pp. 1-20, 1998.

[201] S. Campbell, Fabrication Engineering at the Micro- and Nanoscale, New York: Oxford University Press, 2013.

[202] M. K. Kwak, et al., "Phase-Shift Lithography," in Encyclopedia of Nanotechnology, Springer Science + Business Media, 2014, pp. 1-10.

[203] C. Lu, et al., "Interference lithography: a powerful tool for fabricating periodic structures," Laser Photonics Reviews, vol. 4, no. 4, pp. 568-580, 2010.

[204] C. Waits, et al., "MEMS-based gray-scale lithography," IEEE Proceedings of the 2001 Semiconductor Devices Research Symposium, pp. 182-185, 2001.

[205] K. Chen, et al., "Fabrication of 3D polymer microstructures using electron beam lithography and nanoimprinting technologies," Journal of Micromechanics and Microengineering, vol. 15, pp. 1894-1903, 2005.

[206] G. Barbillon, et al., "Electron beam lithography designed chemical nanosensors based on localized surface plasmon resonance," Elsevier Surface Science,, vol. 601, pp. 5057-5061, 2007.

[207] M. McCord, et al., "Electron beam lithography," SPIE Optical Engineering Press. Handbook of Microlithography, Micromachining and Microfabrication, vol. 1, pp. 197201, 1997.

[208] M. Parikh, "Corrections to proximity effects in electron beam lithography. I. Theory," Journal of Applied Physics, vol. 50, no. 6, 1979.

[209] T. R. Groves, "Efficiency of electron-beam proximity effect correction," J. Vac. Sci. Technol. B Microelectron. Nanometer Struct., vol. 11, no. 6, 1993.

[210] J. R. Brickford, et al., "Hydrogen silsesquioxane on SOI proximity and microloading effects correction from a single 1D characterization sample," J. Vac. Sci. Technol. B Nanotechnol. Microelectron. Mater. Process. Meas. Phenom., vol. 32, no. 6, 2014.

[211] J. A. Currivan, et al., "Polymethyl methacrylate/hydrogen silsesquioxane bilayer resist electron beam lithography process for etching $25 \mathrm{~nm}$ wide magnetic wires," J. Vac. Sci. Technol. B Microelectron. Nanometer Struct., vol. 32, no. 2, 2014.

[212] G. Owen, et al., "Proximity effect correction for electron beam lithography by equalization of background dose," Journal of Applied Physics, vol. 54, no. 6, 1983. 
[213] T. Klimpel, et al., "Model based hybrid proximity effect correction scheme combining dose modulation and shape adjustments," J. Vac. Sci. Technol. B Microelectron. Nanometer Struct., vol. 29, no. 6, 2011.

[214] A. Hohenau, et al., "Electron beam lithography, a helpful tool for nanooptics," Microelectronic Engineering, vol. 83, no. 4-9, pp. 1464-1467, 2006.

[215] C. Vieu, et al., "Electron beam lithography: resolution limits and applications," Applied Surface Science, vol. 164, no. 1, pp. 111-117, 2000.

[216] J. Bergstra, et al., "Algorithms for Hyper-Parameter Optimization," Advances in Neural Information Processing Systems 24 (NIPS), 2011.

[217] I. Malkiel, et al., "Plasmonic nanostructure design and characterization via Deep Learning," Light: Science \& Applications, vol. 7, no. 60, 2018.

[218] S. J.-Zanjani, et al., "Adaptive Genetic Algorithm for Optical Metasurfaces Design," Scientific Reports, vol. 8, no. 11040, 2018.

[219] S. Kessentini, et al., "Particle Swarm Optimization and Evolutionary Methods for Plasmonic Biomedical Applications," IEEE Congress of Evolutionary Computation (CEC), pp. 2315-2320, 2011.

[220] C. R. Nave, "Fabry-Perot Interferometer," HyperPhysics, [Online]. Available: http://hyperphysics.phy-astr.gsu.edu/hbase/phyopt/fabry.html. [Accessed 5 October 2019].

[221] California Institute of Technology, "LIGO Laser Interferometer Gravitational-Wave Observatory," $\quad$ Caltech, $\quad$ MIT, [Online]. Available: https://www.ligo.caltech.edu/video/ligo20160211v1. [Accessed 5 October 2019].

[222] JFC UCL, "Fabry Perot Interferometer," Youtube, [Online]. Available: https://www.youtube.com/watch?v=HkjlfCiEqGE. [Accessed 5 October 2019].

[223] T. W. Ebbesen, et al., "Extraordinary Optical Transmission Through Sub-wavelength Hole Arrays," Nature (London), vol. 391, no. 667, 1998.

[224] R. F. Harrington and D. T. Auckland, "Electromagnetic Transmission Through Narrow Slots in Thick Conducting Screens," IEEE Transactions on Antennas and Propagation, vol. 28, no. 616, 1980.

[225] R. F. Harrington, "Resonant Behavior of a Small Aperture Backed by a Conducting Body," IEEE Transactions on Antennas and Propagation, vol. 30, no. 205, 1982.

[226] Y. Leviatan, et al., "Electromagnetic Transmission Through Apertures in a Cavity in a Thick Conductor," IEEE Transactions on Antennas and Propagation, vol. 30, no. 1153, 1982. 
[227] Y. Takakura, "Optical Resonance in a Narrow Slit in a Thick Metallic Screen," American Physical Society Physical Review Letters, vol. 86, no. 5601, 2001.

[228] F. J. Garcia-Vidal, et al., "Multiple Paths to Enhance Optical Transmission Through a Single Subwavelength Slit," American Physical Society Physical Review Letters, vol. 90, no. 213901, 2003.

[229] X. Shi, et al., "Ultrahigh Light Transmission Through a C-Shaped Nanoaperture," The Optical Society Optics Letters, vol. 28, no. 1320, 2003.

[230] J. Wuenschell and H. K. Kim, "Excitation and Propagation of Surface Plasmons in a Metallic Nanoslit Structure," IEEE Transactions on Nanotechnology, vol. 7, no. 229, 2008.

[231] F. Pardo, et al., "Light Funneling Mechanism Explained by Magnetoelectric Interference," American Physical Society Physical Review Letters, vol. 107, no. 093902, 2011.

[232] L. Sahin, et al., "Enhanced Transmission of Electromagnetic Waves Through Split-Ring Resonator-Shaped Apertures," SPIE Journal of Nanophotonics, vol. 5, no. 051812, 2011.

[233] H. A. Bethe, "Theory of Diffraction by Small Holes," American Physical Society Physical Review, vol. 66, no. 163, 1944.

[234] D. G. Smith, Field Guide to Physical Optics, SPIE, 2013.

[235] Y. Leviatan, "Electromagnetic Coupling Between Two Half-Space Regions Separated by Two Slot-Perforated Parallel Conducting Screens," IEEE Transactions on Microwave Theory and Techniques, vol. 36, no. 44, 1988.

[236] "Dielectrics and Dipoles," MIT OpenCourseWare, 2011. [Online]. Available: https://ocw.mit.edu/courses/electrical-engineering-and-computer-science/6-007electromagnetic-energy-from-motors-to-lasers-spring-2011/lecturenotes/MIT6_007S11_lec15.pdf. [Accessed 20 October 2019].

[237] "Polarization definitions," Ibsen Photonics A/S Ryttermarken, 2019. [Online]. Available: https://ibsen.com/technology-2/polarization-definitions/. [Accessed 20 October 2019].

[238] R. A. Paquin, "Properties of Metals," in Handbook of Optics: Fundamentals, techniques, and design, Volume I, McGraw-Hill, 1994.

[239] C. R. Nave, "Electromagnetic Wave Equation," HyperPhysics, 2016. [Online]. Available: http://hyperphysics.phy-astr.gsu.edu/hbase/Waves/emwv.html. [Accessed 26 January 2020].

[240] X.-T. Kong, et al., "Plasmonic Nanostars with Hotspots for Efficient Generation of Hot Electrons under Solar Illumination," Advanced Optical Materials, vol. 5, pp. 1-10, 2016. 
[241] F. Shan and T. Zhang, "Theoretical study on surface plasmon properties of gold nanostars," IOP Conference Series: Material Science and Engineering, vol. 322, 2018.

[242] B. B. Rajeeva, et al., "Regioselective Localization and Tracking of Biomolecules on Single Gold Nanoparticles," Adv. Sci., vol. 2, pp. 1-8, 2015.

[243] F. Hao, et al., "Plasmon Resonances of a Gold Nanostar," Nano Letters, vol. 7, no. 3, pp. 729-732, 2007.

[244] H. Yuan, et al., "Thousand-fold Enhancement of Single-Molecule Fluorescence Near a Single Gold Nanorod," Angew. Chem. Int. Ed., vol. 52, pp. 1217-1221, 2013.

[245] S. Hu, et al., "Surface plasmon resonance enhancement of photoluminescence intensity and bioimaging application of gold nanorod@ $\mathrm{CdSe} / \mathrm{ZnS}$ quantum dots," Beilstein J. Nanotechnol., vol. 10, pp. 22-31, 2019.

[246] B. J. Nagy, et al., "Near-Field Induced Femtosecond Breakdown of Plasmonic Nanoparticles," Plasmonics, 2019.

[247] Z. Yue, et al., "Intrinsically core-shell plasmonic dielectric nanostructures with ultrahigh refractive index," Sci. Adv., vol. 2, pp. 1-8, 2016.

[248] V. Amendola, et al., "Surface plasmon resonance in gold nanoparticles: a review," $J$. Phys.: Condens. Matter, vol. 29, pp. 1-48, 2017.

[249] I. Makiel, et al., "Plasmonic nanostructure design and characterization via Deep Learning," Light Science \& Application, vol. 7, no. 60, 2018.

[250] S. J.-Zanjani, et al., "Adaptive Genetric Algorithm for Optical Metasurface Design," Scientific Reports, vol. 8, 2018.

[251] S. Kessentini, et al., "Particle Swarm Optimization and Evolutionary Methods for Plasmonic Biomedical Applications," IEEE Congress of Evolutionary Computation, pp. 2315-2320, 2011.

[252] K. Z. Smith, "Development of Periodic Plasmonic Nano-Structures for Enhanced Labeled Bio-Sensing Systems," Ph.D. Proposal, West Virginia University, 2018. 


\section{Chapter 7}

\section{Appendix}

Table 7.1 QDs containing antibody or streptavidin conjugates available from ThermoFisher Scientific showing label name with absorbance (excitation) wavelength ranges and emission peaks [182].

\begin{tabular}{|c|c|c|}
\hline Label & Excitation Range $(\lambda)$ & Emission $(\lambda)$ \\
\hline Qdot@ 525 & $<525 \mathrm{~nm}$ & $525 \mathrm{~nm}$ \\
\hline Qdot@ 565 & $<565 \mathrm{~nm}$ & $565 \mathrm{~nm}$ \\
\hline Qdot@ 585 & $<585 \mathrm{~nm}$ & $585 \mathrm{~nm}$ \\
\hline Qdot ${ }^{\circledR} 605$ & $<605 \mathrm{~nm}$ & $605 \mathrm{~nm}$ \\
\hline Qdot ${ }^{\circledR} 625$ & $<625 \mathrm{~nm}$ & $625 \mathrm{~nm}$ \\
\hline Qdot@ 655 & $<655 \mathrm{~nm}$ & $655 \mathrm{~nm}$ \\
\hline Qdot@ 705 & $<705 \mathrm{~nm}$ & $705 \mathrm{~nm}$ \\
\hline Qdot@ 800 & $<800 \mathrm{~nm}$ & $800 \mathrm{~nm}$ \\
\hline
\end{tabular}

Table 7.2 QD cell labeling kits available from ThermoFisher Scientific showing label name with excitation wavelength ranges and emission

\begin{tabular}{|c|c|c|}
\hline Label & Excitation Peak Range $(\lambda)$ & Emission $(\lambda)$ \\
\hline Qtracker® 525 Cell Labeling Kit & $405 \mathrm{~nm}-485 \mathrm{~nm}$ & $525 \mathrm{~nm}$ \\
\hline Qtracker® 565 Cell Labeling Kit & $405 \mathrm{~nm}-525 \mathrm{~nm}$ & $565 \mathrm{~nm}$ \\
\hline Qtracker® 585 Cell Labeling Kit & $405 \mathrm{~nm}-545 \mathrm{~nm}$ & $585 \mathrm{~nm}$ \\
\hline Qtracker® 605 Cell Labeling Kit & $405 \mathrm{~nm}-565 \mathrm{~nm}$ & $605 \mathrm{~nm}$ \\
\hline Qtracker® 625 Cell Labeling Kit & $405 \mathrm{~nm}-585 \mathrm{~nm}$ & $625 \mathrm{~nm}$ \\
\hline Qtracker® 655 Cell Labeling Kit & $405 \mathrm{~nm}-615 \mathrm{~nm}$ & $655 \mathrm{~nm}$ \\
\hline Qtracker® 705 Cell Labeling Kit & $405 \mathrm{~nm}-665 \mathrm{~nm}$ & $705 \mathrm{~nm}$ \\
\hline Qtracker® 800 Cell Labeling Kit & $405 \mathrm{~nm}-760 \mathrm{~nm}$ & $800 \mathrm{~nm}$ \\
\hline
\end{tabular}

Table 7.3 Non-targeted fluorescent QDs available from ThermoFisher Scientific showing label name with excitation wavelength ranges and emission peaks for in vivo imaging of tumor and non-tumor vasculature in mice [182].

\begin{tabular}{|c|c|c|}
\hline \multicolumn{2}{|c|}{ emission peaks for in vivo imaging of tumor and non-tumor vasculature in mice [182]. } \\
\hline Label & Excitation Peak Range $(\lambda)$ & Emission Peak $(\lambda)$ \\
\hline Qtracker® 565 non-targeted QDs & $405 \mathrm{~nm}-525 \mathrm{~nm}$ & $565 \mathrm{~nm}$ \\
\hline Qtracker® 655 non-targeted QDs & $405 \mathrm{~nm}-615 \mathrm{~nm}$ & $655 \mathrm{~nm}$ \\
\hline Qtracker® 705 non-targeted QDs & $405 \mathrm{~nm}-665 \mathrm{~nm}$ & $705 \mathrm{~nm}$ \\
\hline Qtracker® 800 non-targeted QDs & $405 \mathrm{~nm}-760 \mathrm{~nm}$ & $800 \mathrm{~nm}$ \\
\hline
\end{tabular}

Table 7.4 Cadmium-selenide (CdSe) core QDs coated with octadecylamine (ODA) stabilizing ligand in toluene available from NNCrystal, US Corporation showing label product number, absorbance (excitation) and emission peaks, reported quantum yield, and concentration in solution

\begin{tabular}{|c|c|c|c|c|}
\hline Label & Excitation Peak Range $(\lambda)$ & Emission Peak Range $(\lambda)$ & Quantum Yield & $\begin{array}{c}\text { Concentration } \\
(\text { nmol/mg) }\end{array}$ \\
\hline CSE480 & $480 \pm 10 \mathrm{~nm}$ & $485 \mathrm{~nm}-510 \mathrm{~nm}$ & $>0.30$ & 20.0 \\
\hline CSE500 & $500 \pm 10 \mathrm{~nm}$ & $505 \mathrm{~nm}-530 \mathrm{~nm}$ & $>0.30$ & 20.0 \\
\hline CSE520 & $520 \pm 10 \mathrm{~nm}$ & $525 \mathrm{~nm}-550 \mathrm{~nm}$ & $>0.30$ & 20.0 \\
\hline CSE540 & $540 \pm 10 \mathrm{~nm}$ & $545 \mathrm{~nm}-570 \mathrm{~nm}$ & $>0.30$ & 20.0 \\
\hline CSE560 & $560 \pm 10 \mathrm{~nm}$ & $565 \mathrm{~nm}-570 \mathrm{~nm}$ & $>0.30$ & 15.0 \\
\hline CSE580 & $580 \pm 10 \mathrm{~nm}$ & $585 \mathrm{~nm}-610 \mathrm{~nm}$ & $>0.30$ & 4.0 \\
\hline CSE590 & $590 \pm 10 \mathrm{~nm}$ & $595 \mathrm{~nm}-620 \mathrm{~nm}$ & $>0.30$ & 3.0 \\
\hline CSE600 & $600 \pm 10 \mathrm{~nm}$ & $610 \mathrm{~nm}-625 \mathrm{~nm}$ & $>0.30$ & 2.0 \\
\hline CSE610 & $610 \pm 10 \mathrm{~nm}$ & $620 \mathrm{~nm}-635 \mathrm{~nm}$ & $>0.30$ & 1.0 \\
\hline CSE640 & $620 \pm 10 \mathrm{~nm}$ & $615 \mathrm{~nm}-650 \mathrm{~nm}$ & $>0.30$ & 1.0 \\
\hline
\end{tabular}


Table 7.5 Cadmium-selenide/zinc-sulfide $(\mathrm{CdSe} / \mathrm{ZnS})$ core-shell QDs coated with carboxylic acid stabilizing ligand in water available from NNCrystal, US Corporation showing label product number, absorbance (excitation) and emission peaks, reported quantum yield, and concentration in solultion [184]

\begin{tabular}{|c|c|c|c|c|}
\hline Label-Emission Color & Excitation Range $(\boldsymbol{\lambda})$ & Emission Peak Range $(\boldsymbol{\lambda})$ & Quantum Yield & $\begin{array}{c}\text { Concentration } \\
(\mathbf{n m o l} / \mathbf{m g})\end{array}$ \\
\hline CZW-Dark Red & $<700 \mathrm{~nm}$ & $640 \mathrm{~nm}-660 \mathrm{~nm}$ & $\geq 0.20$ \\
\hline CZW-Green & $<600 \mathrm{~nm}$ & $520 \mathrm{~nm}-550 \mathrm{~nm}$ & $\geq 0.20$ \\
\hline CZW-Orange & $<625 \mathrm{~nm}$ & $590 \mathrm{~nm}-615 \mathrm{~nm}$ & $\geq 0.20$ \\
\hline CZW-Red & $<675 \mathrm{~nm}$ & $620 \mathrm{~nm}-640 \mathrm{~nm}$ & 1.0 \\
\hline CZW-Yellow & $<600 \mathrm{~nm}$ & $575 \mathrm{~nm}-585 \mathrm{~nm}$ & 1.0 \\
\hline
\end{tabular}

Table 7.6 Cadmium-sulfide (CdS) core QDs coated with oleic acid stabilizing ligand in toluene available from NNCrystal, US Corporation showing label product number, absorbance (excitation) and emission peaks, reported quantum yield, and concentration in solultion [184].

\begin{tabular}{|c|c|c|c|c|}
\hline Label & Excitation Peak Range $(\lambda)$ & Emission Peak Range $(\lambda)$ & Quantum Yield & $\begin{array}{c}\text { Concentration } \\
\text { (nmol/mg) }\end{array}$ \\
\hline CS360 & $360 \pm 10 \mathrm{~nm}$ & $365 \mathrm{~nm}-390 \mathrm{~nm}$ & $\geq 0.20$ & 20.0 \\
\hline CS380 & $380 \pm 10 \mathrm{~nm}$ & $385 \mathrm{~nm}-410 \mathrm{~nm}$ & $\geq 0.20$ & 15.0 \\
\hline CS400 & $400 \pm 10 \mathrm{~nm}$ & $405 \mathrm{~nm}-430 \mathrm{~nm}$ & $\geq 0.20$ & 10.0 \\
\hline CS420 & $420 \pm 10 \mathrm{~nm}$ & $425 \mathrm{~nm}-450 \mathrm{~nm}$ & $\geq 0.20$ & 6.0 \\
\hline CS440 & $440 \pm 10 \mathrm{~nm}$ & $445 \mathrm{~nm}-470 \mathrm{~nm}$ & $\geq 0.20$ & 4.5 \\
\hline CS460 & $460 \pm 10 \mathrm{~nm}$ & $465 \mathrm{~nm}-490 \mathrm{~nm}$ & $\geq 0.20$ & 3.0 \\
\hline
\end{tabular}

Table 7.7 Cadmium-telluride (CdTe) core coated with octadecylphosphoric (ODPA) acid in toluene available from NNCrystal, US Corporation showing label product number, absorbance (excitation) and emission peaks, reported quantum yield, and concentration in solultion [184].

\begin{tabular}{|c|c|c|c|c|}
\hline Label & Excitation Peak Range ( $\lambda)$ & Emission Peak Range ( $\boldsymbol{\lambda})$ & $\begin{array}{c}\text { Quantum Yield } \\
\text { (nmol/mg) }\end{array}$ \\
\hline CT640 & $<615 \mathrm{~nm}$ & $640 \pm 10 \mathrm{~nm}$ & $\geq 0.20$ \\
\hline
\end{tabular}

Table 7.8 Cadmium-telluride/selenium-sulfide (CdTe/SeS) type-II core-shell QDs coated with octadecylamine (ODA) stabilizing ligand in toluene available from NNCrystal, US Corporation showing label product number, absorbance (excitation) and emission peaks, reported quantum yield, concentration in solultion, and full-width half-maximum (FWHM) [184].

\begin{tabular}{|c|c|c|c|c|c|}
\hline Label & Excitation Range $(\lambda)$ & $\begin{array}{c}\text { Emission Peak Range } \\
(\lambda)\end{array}$ & $\begin{array}{l}\text { Quantum } \\
\text { Yield }\end{array}$ & $\begin{array}{l}\text { Concentration } \\
\text { (nmol/mg) }\end{array}$ & FWHM \\
\hline CTSS700 & $<1,000 \mathrm{~nm}$ & $700 \pm 50 \mathrm{~nm}$ & +0.30 & 3.7 & $61 \mathrm{~nm}$ \\
\hline CTSS800 & $<1,000 \mathrm{~nm}$ & $800 \pm 50 \mathrm{~nm}$ & +0.30 & 2.9 & $105 \mathrm{~nm}$ \\
\hline CTSS900 & $<1,000 \mathrm{~nm}$ & $900 \pm 50 \mathrm{~nm}$ & +0.30 & 2.4 & $132 \mathrm{~nm}$ \\
\hline
\end{tabular}

Table 7.9 Cadmium-selenide/zinc-sulfide $(\mathrm{CdSe} / \mathrm{ZnS})$ QD microspheres coated with carboxylic acid or streptavidin stabilizing ligands in water available from NNCrystal, US Corporation showing label product number, absorbance (excitation) and emission peaks, reported quantum yield,

\begin{tabular}{|c|c|c|c|c|}
\hline Label & $\begin{array}{c}\text { Excitation Peak Range } \\
(\boldsymbol{\lambda})\end{array}$ & $\begin{array}{c}\text { Emission Peak Range } \\
(\boldsymbol{\lambda})\end{array}$ & $\begin{array}{c}\text { Quantum Yield } \\
\text { Concentration }(\boldsymbol{\mu M})\end{array}$ & \multirow{2}{*}{ FWHM } \\
\hline QDM-525 & $300 \mathrm{~nm}-450 \mathrm{~nm}$ & $525 \pm 10 \mathrm{~nm}$ & $\geq 0.60$ & 1.0 \\
\hline QDM-565 & $300 \mathrm{~nm}-450 \mathrm{~nm}$ & $565 \pm 10 \mathrm{~nm}$ & $\geq 0.60$ & $\leq 30 \mathrm{~nm}$ \\
\hline QDM-585 & $300 \mathrm{~nm}-450 \mathrm{~nm}$ & $585 \pm 10 \mathrm{~nm}$ & $\geq 0.60$ & 1.0 \\
\hline QDM-610 & $300 \mathrm{~nm}-450 \mathrm{~nm}$ & $610 \pm 10 \mathrm{~nm}$ & $\geq 0.60$ & 1.0 \\
\hline QDM-625 & $300 \mathrm{~nm}-450 \mathrm{~nm}$ & $625 \pm 10 \mathrm{~nm}$ & $\geq 0.60$ & 1.0 \\
\hline QDM-655 & $300 \mathrm{~nm}-450 \mathrm{~nm}$ & $655 \pm 10 \mathrm{~nm}$ & $\geq 0.60$ & 1.0 \\
\hline
\end{tabular}


Table 7.10 Lead-selenide ( $\mathrm{PbSe}$ ) core QDs coated with oleic acid stabilizing ligand in toluene available from NNCrystal, US Corporation showing label product number, absorbance (excitation) and emission peaks, reported quantum yield, concentration in solultion, and full-width

\begin{tabular}{|c|c|c|c|c|c|}
\hline Label & Excitation Range $(\lambda)$ & $\begin{array}{c}\text { Emission Peak Range } \\
(\lambda)\end{array}$ & $\begin{array}{c}\text { Quantum } \\
\text { Yield }\end{array}$ & $\begin{array}{c}\text { Concentration } \\
(\mathbf{n m o l} / \mathbf{m g})\end{array}$ & FWHM \\
\hline $\mathrm{PbSe} 1200$ & $<1,550 \mathrm{~nm}$ & $1,200 \pm 50 \mathrm{~nm}$ & +0.0 & 9.0 & $137 \mathrm{~nm}$ \\
\hline $\mathrm{PbSe} 1300$ & $<1,600 \mathrm{~nm}$ & $1,300 \pm 50 \mathrm{~nm}$ & +0.40 & 6.0 & $138 \mathrm{~nm}$ \\
\hline $\mathrm{PbSe} 1400$ & $<1,650 \mathrm{~nm}$ & $1,400 \pm 50 \mathrm{~nm}$ & +0.40 & 4.3 & $136 \mathrm{~nm}$ \\
\hline $\mathrm{PbSe} 1500$ & $<1,650 \mathrm{~nm}$ & $1,500 \pm 50 \mathrm{~nm}$ & +0.40 & 3.1 & $138 \mathrm{~nm}$ \\
\hline $\mathrm{PbSe} 1600$ & $<1,800 \mathrm{~nm}$ & $1,600 \pm 50 \mathrm{~nm}$ & +0.40 & 2.4 & $139 \mathrm{~nm}$ \\
\hline $\mathrm{PbSe} 1700$ & $<2,000 \mathrm{~nm}$ & $1,700 \pm 50 \mathrm{~nm}$ & +0.40 & 1.8 & $144 \mathrm{~nm}$ \\
\hline $\mathrm{PbSe} 1800$ & $<2,000 \mathrm{~nm}$ & $1,800 \pm 50 \mathrm{~nm}$ & +0.40 & 1.4 & $145 \mathrm{~nm}$ \\
\hline
\end{tabular}

Table 7.11 Lead-sulfide $(\mathrm{PbS})$ core QDs coated with oleic acid stabilizing ligand in toluene available from NNCrystal, US Corporation showing label product number, absorbance (excitation) and emission peaks, reported quantum yield, concentration in solultion, and full-width half-

\begin{tabular}{|c|c|c|c|c|c|}
\hline Label & Excitation Range $(\lambda)$ & $\begin{array}{c}\text { Emission Peak Range } \\
(\lambda)\end{array}$ & Quantum Yield & $\begin{array}{c}\text { Concentration } \\
(\mathbf{n m o l} / \mathbf{m g})\end{array}$ & FWHM \\
\hline $\mathrm{PbS} 900$ & $<1,050 \mathrm{~nm}$ & $900 \pm 50 \mathrm{~nm}$ & +040 & 10.6 & $122 \mathrm{~nm}$ \\
\hline $\mathrm{PbS} 1000$ & $<1,100 \mathrm{~nm}$ & $1,000 \pm 50 \mathrm{~nm}$ & +0.40 & 8.4 & $128 \mathrm{~nm}$ \\
\hline $\mathrm{PbS} 1100$ & $<1,200 \mathrm{~nm}$ & $1,100 \pm 50 \mathrm{~nm}$ & +0.40 & 5.6 & $132 \mathrm{~nm}$ \\
\hline $\mathrm{PbS} 1200$ & $<1,350 \mathrm{~nm}$ & $1,200 \pm 50 \mathrm{~nm}$ & +0.40 & 4.0 & $136 \mathrm{~nm}$ \\
\hline $\mathrm{PbS} 1300$ & $<1,400 \mathrm{~nm}$ & $1,300 \pm 50 \mathrm{~nm}$ & +0.40 & 3.5 & $136 \mathrm{~nm}$ \\
\hline $\mathrm{PbS} 1400$ & $<1,500 \mathrm{~nm}$ & $1,400 \pm 50 \mathrm{~nm}$ & +0.40 & 2.7 & $137 \mathrm{~nm}$ \\
\hline $\mathrm{PbS} 1500$ & $<1,600 \mathrm{~nm}$ & $1,500 \pm 50 \mathrm{~nm}$ & +0.40 & 2.1 & $131 \mathrm{~nm}$ \\
\hline $\mathrm{PbS} 1600$ & $<1,700 \mathrm{~nm}$ & $1,600 \pm 50 \mathrm{~nm}$ & +0.40 & 1.6 & $138 \mathrm{~nm}$ \\
\hline
\end{tabular}

Table 7.12 Copper-indium-sulfide/zinc-sulfide (CuInS/ZnS) core-shell QDs coated with oleic acid/oleyamine stabilizing ligands in toluene available from NNCrystal, US Corporation showing label product number, absorbance (excitation) and emission peaks, reported quantum yield,

\begin{tabular}{|c|c|c|c|c|c|}
\hline \multicolumn{7}{|c|}{ concentration in solultion, and full-width half-maximum (FWHM) [184]. } \\
\hline Label & Excitation Range $(\lambda)$ & $\begin{array}{c}\text { Emission Peak Range } \\
(\lambda)\end{array}$ & $\begin{array}{c}\text { Concentration } \\
(\mathbf{n m o l} / \mathbf{m g})\end{array}$ & FWHM \\
\hline CIS530 & $<700 \mathrm{~nm}$ & $530 \pm 15 \mathrm{~nm}$ & 0.20 & 9.1 & $69 \mathrm{~nm}$ \\
\hline CIS560 & $<700 \mathrm{~nm}$ & $560 \pm 15 \mathrm{~nm}$ & 0.20 & 7.1 & $84 \mathrm{~nm}$ \\
\hline CIS590 & $<700 \mathrm{~nm}$ & $590 \pm 15 \mathrm{~nm}$ & 0.20 & 5.2 & $92 \mathrm{~nm}$ \\
\hline CIS620 & $<700 \mathrm{~nm}$ & $620 \pm 15 \mathrm{~nm}$ & 0.20 & 3.5 & $99 \mathrm{~nm}$ \\
\hline CIS650 & $<700 \mathrm{~nm}$ & $650 \pm 15 \mathrm{~nm}$ & 0.20 & 1.7 & $100 \mathrm{~nm}$ \\
\hline CIS700 & $<700 \mathrm{~nm}$ & $700 \pm 15 \mathrm{~nm}$ & 0.20 & 1.2 & $120 \mathrm{~nm}$ \\
\hline CIS750 & $<700 \mathrm{~nm}$ & $750 \pm 15 \mathrm{~nm}$ & 0.20 & 1.0 & $111 \mathrm{~nm}$ \\
\hline CIS800 & $<700 \mathrm{~nm}$ & $800 \pm 15 \mathrm{~nm}$ & 0.10 & 0.95 & $102 \mathrm{~nm}$ \\
\hline CIS850 & $<700 \mathrm{~nm}$ & $850 \pm 15 \mathrm{~nm}$ & 0.10 & 0.91 & $100 \mathrm{~nm}$ \\
\hline
\end{tabular}

Table 7.13 Copper-indium-sulfide/zinc-sulfide (CuInS/ZnS) core-shell QDs coated with carboxylic acid stabilizing ligand in water available from NNCrystal, US Corporation showing label product number, absorbance (excitation) and emission peaks, reported quantum yield, concentration in solultion, and full-width half-maximum (FWHM) [184].

\begin{tabular}{|c|c|c|c|c|c|}
\hline Label & Excitation Range $(\lambda)$ & $\begin{array}{c}\text { Emission Peak } \\
\text { Range }(\lambda)\end{array}$ & Quantum Yield & $\begin{array}{c}\text { Concentration } \\
(\mathbf{n m o l} / \mathbf{m g})\end{array}$ & FWHM \\
\hline CISW530 & $<700 \mathrm{~nm}$ & $530 \pm 10 \mathrm{~nm}$ & $>0.15$ & 9.1 & $69 \mathrm{~nm}$ \\
\hline CISW560 & $<700 \mathrm{~nm}$ & $560 \pm 10 \mathrm{~nm}$ & $>0.15$ & 7.1 & $84 \mathrm{~nm}$ \\
\hline CISW590 & $<700 \mathrm{~nm}$ & $590 \pm 10 \mathrm{~nm}$ & $>0.15$ & 5.2 & $92 \mathrm{~nm}$ \\
\hline CISW620 & $<700 \mathrm{~nm}$ & $620 \pm 10 \mathrm{~nm}$ & $>0.15$ & 3.5 & $99 \mathrm{~nm}$ \\
\hline CISW650 & $<700 \mathrm{~nm}$ & $650 \pm 10 \mathrm{~nm}$ & $>0.15$ & 1.7 & $100 \mathrm{~nm}$ \\
\hline CISW700 & $<700 \mathrm{~nm}$ & $700 \pm 10 \mathrm{~nm}$ & $>0.15$ & 1.2 & $120 \mathrm{~nm}$ \\
\hline CISW750 & $<700 \mathrm{~nm}$ & $750 \pm 10 \mathrm{~nm}$ & $>0.15$ & 1.0 & $111 \mathrm{~nm}$ \\
\hline CIS800 & $<700 \mathrm{~nm}$ & $800 \pm 15 \mathrm{~nm}$ & $>0.15$ & 0.95 & $102 \mathrm{~nm}$ \\
\hline CIS850 & $<700 \mathrm{~nm}$ & $850 \pm 15 \mathrm{~nm}$ & $>0.15$ & 0.91 & $100 \mathrm{~nm}$ \\
\hline
\end{tabular}


Table 7.14 Indium-phosphide/zinc-sulfide ( $\mathrm{InP} / \mathrm{ZnS})$ core-shell QDs coated with oleyamine stabilizing ligand in toluene available from NNCrystal, US Corporation showing label product number, absorbance (excitation) and emission peaks, reported quantum yield, concentration in solultion, and full-width half-maximum (FWHM) [184].

\begin{tabular}{|c|c|c|c|c|}
\hline Label & Excitation Peak Range $(\lambda)$ & Emission Peak Range $(\lambda)$ & Quantum Yield & $\begin{array}{c}\text { Concentration } \\
(\mathbf{n m o l} / \mathbf{m g})\end{array}$ \\
\hline INP530 & $<575 \mathrm{~nm}$ & $530 \pm 15 \mathrm{~nm}$ & $>0.20$ & 6.5 \\
\hline INP560 & $<600 \mathrm{~nm}$ & $560 \pm 15 \mathrm{~nm}$ & $>0.20$ & 5.5 \\
\hline INP590 & $<650 \mathrm{~nm}$ & $590 \pm 15 \mathrm{~nm}$ & $>0.20$ & 4.0 \\
\hline INP620 & $<700 \mathrm{~nm}$ & $620 \pm 15 \mathrm{~nm}$ & $>0.20$ & 3.0 \\
\hline INP650 & $<725 \mathrm{~nm}$ & $650 \pm 15 \mathrm{~nm}$ & $>0.10$ & 3.0 \\
\hline
\end{tabular}

Table 7.15 Indium-phosphide/zinc-sulfide (InP/ZnS) core-shell QDs coated with carboxylic acid stabilizing ligand in water available from NNCrystal, US Corporation showing label product number, absorbance (excitation) and emission peaks, reported quantum yield, concentration in solultion, and full-width half-maximum (FWHM) [184].

\begin{tabular}{|c|c|c|c|c|c|}
\hline Label & Excitation Range $(\lambda)$ & $\begin{array}{c}\text { Emission Peak Range } \\
(\lambda)\end{array}$ & Quantum Yield & $\begin{array}{c}\text { Concentration } \\
(\mathbf{n m o l} / \mathbf{m g})\end{array}$ & FWHM \\
\hline INPW530 & $<700 \mathrm{~nm}$ & $530 \pm 15 \mathrm{~nm}$ & $>0.10$ & 6.5 & $52 \mathrm{~nm}$ \\
\hline INPW560 & $<700 \mathrm{~nm}$ & $560 \pm 15 \mathrm{~nm}$ & $>0.10$ & 5.5 & $50 \mathrm{~nm}$ \\
\hline INPW590 & $<700 \mathrm{~nm}$ & $590 \pm 15 \mathrm{~nm}$ & $>0.10$ & 4.0 & $55 \mathrm{~nm}$ \\
\hline INPW620 & $<700 \mathrm{~nm}$ & $620 \pm 15 \mathrm{~nm}$ & $>0.10$ & 3.0 & $57 \mathrm{~nm}$ \\
\hline INPW650 & $<700 \mathrm{~nm}$ & $650 \pm 15 \mathrm{~nm}$ & $>0.10$ & 3.0 & $59 \mathrm{~nm}$ \\
\hline
\end{tabular}

Table 7.16 Manganese-doped zinc-selenide (Mn: ZnSe, or D-dots) QDs coated with oleyamine stabilizing ligand in toluene available from NNCrystal, US Corporation showing label product number, absorbance (excitation) and emission peaks, reported quantum yield, concentration in

\begin{tabular}{|c|c|c|c|c|c|}
\hline \multicolumn{5}{|c|}{ solultion, and full-width half-maximum (FWHM) [184]. } \\
\hline \multirow{2}{*}{ Label } & Excitation Range $(\lambda)$ & $\begin{array}{c}\text { Emission Peak Range } \\
(\lambda)\end{array}$ & Quantum Yield & $\begin{array}{c}\text { Concentration } \\
(\mathbf{n m o l} / \mathbf{m g})\end{array}$ & FWHM \\
\hline DD600 & $<700 \mathrm{~nm}$ & $600 \pm 10 \mathrm{~nm}$ & $>0.50$ & 2.0 & $60 \mathrm{~nm}$ \\
\hline DD620 & $<700 \mathrm{~nm}$ & $620 \pm 10 \mathrm{~nm}$ & $>0.50$ & 1.5 & $70 \mathrm{~nm}$ \\
\hline
\end{tabular}

Table 7.17 Manganese-doped zinc-selenide (Mn: ZnSe, or D-dots) QDs coated with carboxylic acid stabilizing ligand in water available from NNCrystal, US Corporation showing label product number, absorbance (excitation) and emission peaks, reported quantum yield, concentration in

\begin{tabular}{|c|c|c|c|c|c|}
\hline Label & Excitation Range $(\lambda)$ & $\begin{array}{c}\text { Emission Peak Range } \\
(\lambda)\end{array}$ & Quantum Yield & $\begin{array}{c}\text { Concentration } \\
(\mathbf{n m o l} / \mathbf{m g})\end{array}$ & FWHM \\
\hline DDW600 & $<700 \mathrm{~nm}$ & $600 \pm 10 \mathrm{~nm}$ & $>0.30$ & 2.0 & $60 \mathrm{~nm}$ \\
\hline DDW620 & $<700 \mathrm{~nm}$ & $620 \pm 10 \mathrm{~nm}$ & $>0.30$ & 1.5 & $70 \mathrm{~nm}$ \\
\hline
\end{tabular}

